\title{
ALEUTIAN SEISMIC PROGRAM SEISMOLOGICAL BULLETIN NOVEMBER 1970
}

\author{
U.S. DEPARTMENT OF COMMERCE \\ NATIONAL OCEANIC AND ATMOSPHERIC ADMINISTRATION \\ NATIONAL OCEAN SURVEY \\ ROCKVILLE, MARYLANO 20852
}

\author{
JUNE 1971
}
DREPARED FOP THE U.S. ATOMIC ENERGY COMMISSION NEVADA OPERATIONS OFFICE UNDER CONTRACT AT $(29-2)-746$




\section{DISCLAIMER}

This report was prepared as an account of work sponsored by an agency of the United States Government. Neither the United States Government nor any agency Thereof, nor any of their employees, makes any warranty, express or implied, or assumes any legal liability or responsibility for the accuracy, completeness, or usefulness of any information, apparatus, product, or process disclosed, or represents that its use would not infringe privately owned rights. Reference herein to any specific commercial product, process, or service by trade name, trademark, manufacturer, or otherwise does not necessarily constitute or imply its endorsement, recommendation, or favoring by the United States Government or any agency thereof. The views and opinions of authors expressed herein do not necessarily state or reflect those of the United States Government or any agency thereof. 


\section{DISCLAIMER}

Portions of this document may be illegible in electronic image products. Images are produced from the best available original document. 
THIS REPORT WAS PREPARED AS AN ACCOUNT OF WORK SPONSORED BY THE UNITED STATES G IVERNMENT. NEITHER THE UNITED.STATES NOR THE UNITED STATES ATOMIC ENERGY COMMISSION, NOR ANY OF THEIR. EMPLOYEES, NOR ANY OF THEIR CONTRACTORS, SUBCONTRACTORS, OR THEIR EMPLOYEES, MAKES ANY WARRANTY, EXPRESS OR IMPLIED, OR ASSUMES ANY LEGAL LIAPILITY OP RESPONSIBILITY FOR THF ACCURACY, COMPLETENESS OR USEFULNESS DF ANY INFORMATION, APPAPATUS, PRODUC OR PROCESS DISCLOSED, OR REPRESENTS THAT ITS USE WOULD NOT INFRINGE PRIVATELY-OWNED RIGHTS.

AVAILAELE FROM THE

NATIONAL TECHNICAL INFORMATION SERVICE U.S. DEPARTMENT OF COMMERCE SPRINGFIELD, VIRGINIA 22151 
U.S. DEPARTMENT OF COMMERCE

NATIONAL OCEANIC AND ATMOSPHERIC ADMINISTRATION NATIONAL OCEAN SURVEY ROCKVILLE, MARYLAND 20852

$\Lambda$

\author{
JUNE 1971
}

\title{
PREPARED FOR THE U.S. ATOMIC ENERGY COMMISSION NEVADA OPERATIONS OFFICE UNDER CONTRACT AT (29-2)-746
}


SEISMOL OGICAL BULLETIN

THE PURPOSE OF THIS BULLETIN IS TO COLLECT AND DISTRIBUTE PRELIMINARY DATA ACOUIRED FROM AN ALEUTIAN NETHORK OF SEISHIC STATIONS IN ORDER TO FACILITATE CURRENT STUDIES OF EARTHQUAKE OCCURRENCE IN THE ALEUTIAN ISLANOS. RECORD ARRIVAL TIMES AND AMPLITUDES ARE SCALED BY THE SPECIAL PROJECTS PARTY, LAS VEGAS, NEVADA. FINAL REDUCTION AND COLLATION OF OATA TO EVENT HYPOCENTERS AND DERIVATION OF RELATED PARAMETERS ARE PERFORMED BY THE SEISMOLOGY DIVISION, ROCKVILLE, MARYLAND. THIS COMPILATION IS PRELIMINARY AND MAY BE SUBJECT TO LATER REVISION ON THE BASIS OF CURRENT RESEARCH. 


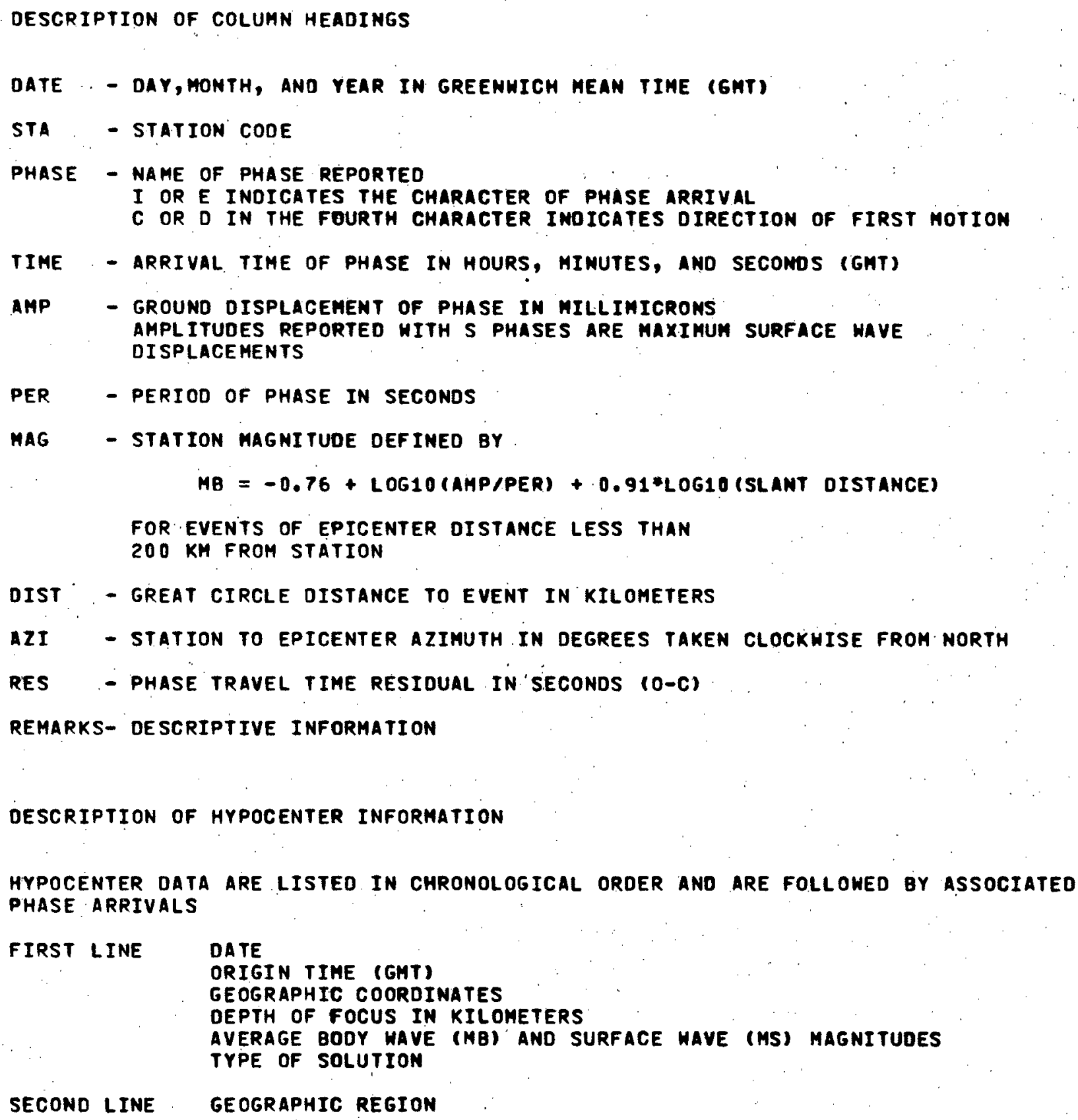


ALEUTIAN SEISMIC NETHORK

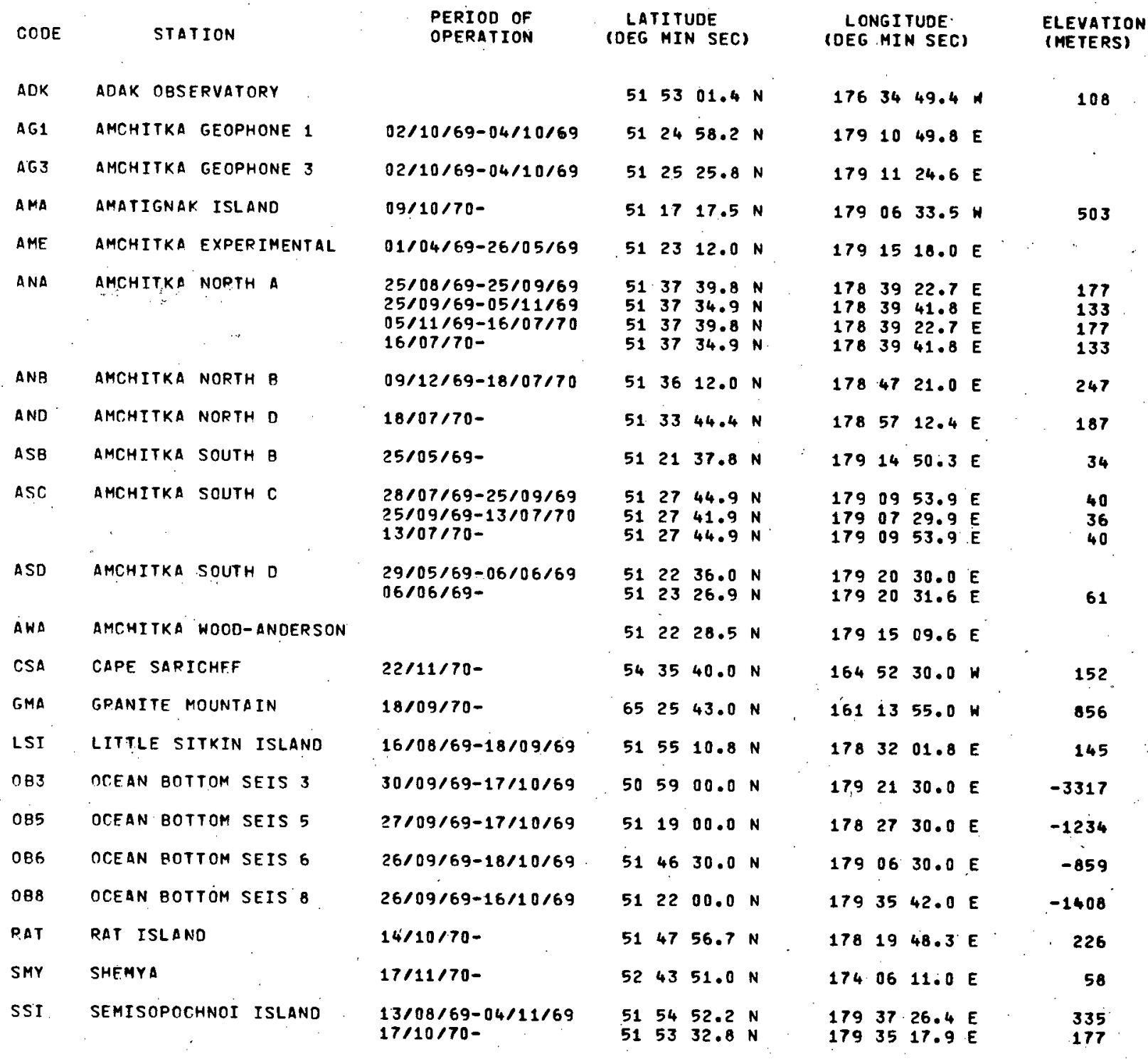




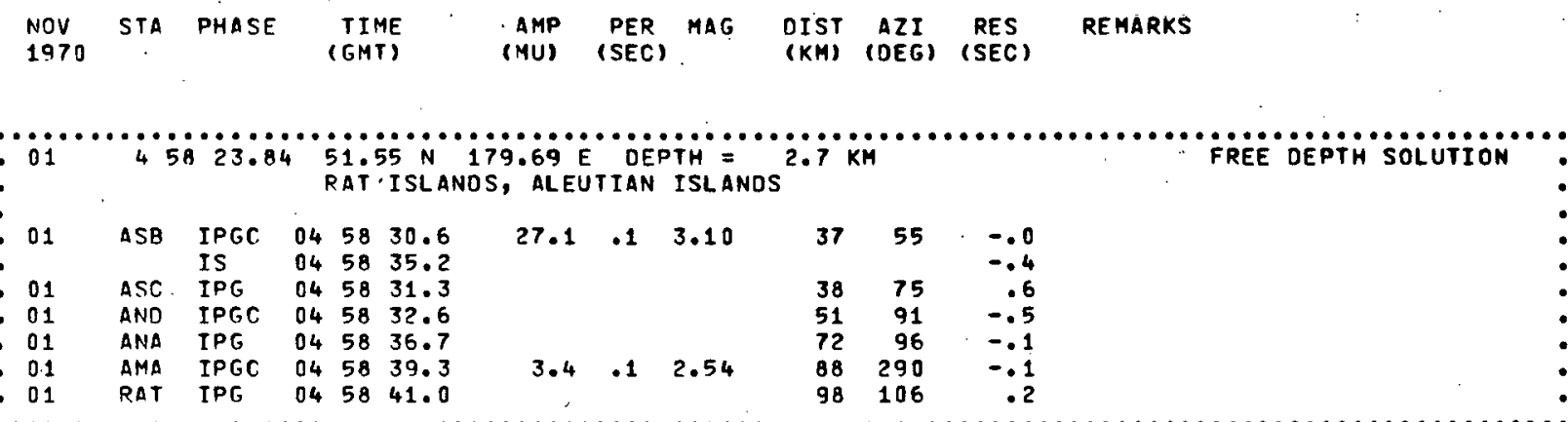

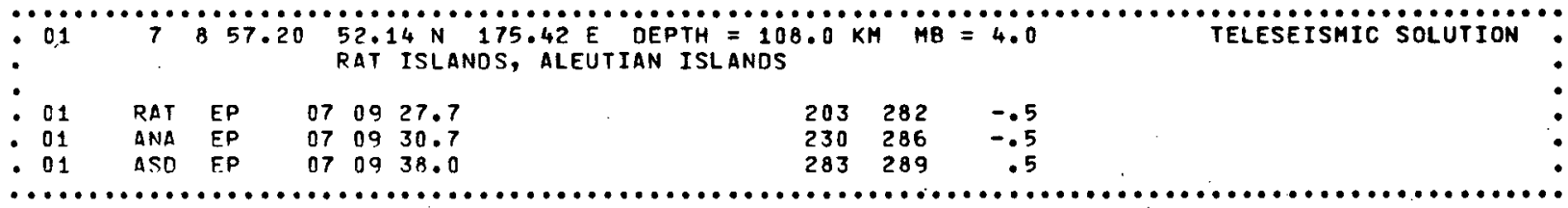

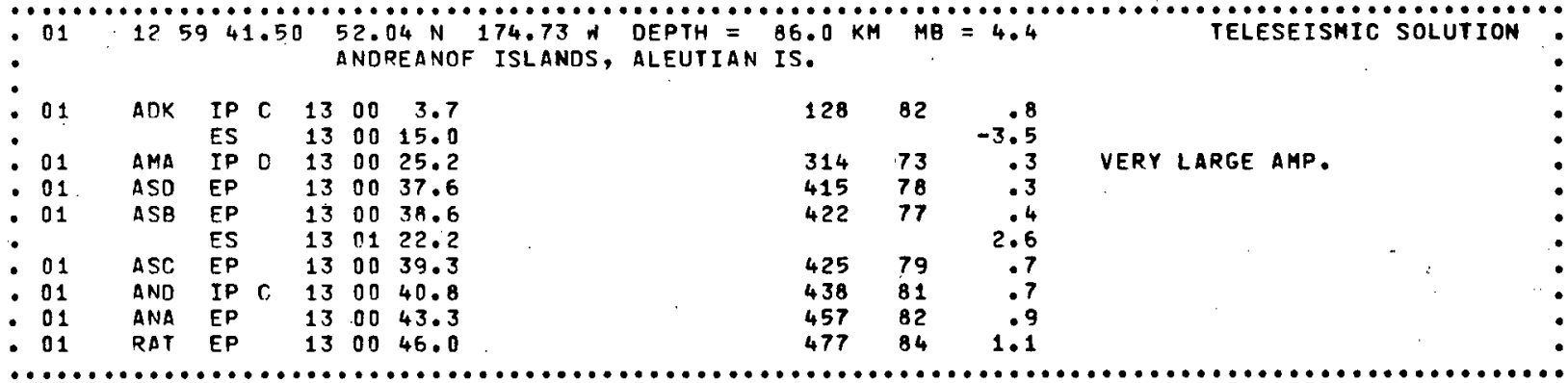

\begin{tabular}{|c|c|c|c|c|c|c|c|c|c|c|}
\hline $\begin{array}{l}: 01 \\
: 01 \\
: 01 \\
0 \\
01\end{array}$ & $\begin{array}{l}\text { AND } \\
\text { ASC } \\
\text { ASB } \\
\text { ASD }\end{array}$ & $\begin{array}{l}\text { IPGD } \\
\text { IS } \\
\text { IPG } \\
\text { EPG } \\
\text { IPGD }\end{array}$ & $\begin{array}{ll}13 & 5 \\
13 & 5 \\
13 & 5 \\
13 & 5 \\
13 & 5\end{array}$ & $\begin{array}{rr}51 & 52.5 \\
52 & .2 \\
51 & 53.5 \\
51 & 53.8 \\
51 & 54.0\end{array}$ & $\begin{array}{l}2.4 \\
2.4\end{array}$ & $\begin{array}{l}.1 \\
.1\end{array}$ & $\begin{array}{l}2.35 \\
2.36\end{array}$ & $\begin{array}{l}14 \\
29 \\
39 \\
43\end{array}$ & $\begin{array}{l}253 \\
284 \\
298 \\
290\end{array}$ & $\begin{array}{r}-.1 \\
-.0 \\
.3 \\
.0 \\
-.1\end{array}$ \\
\hline
\end{tabular}

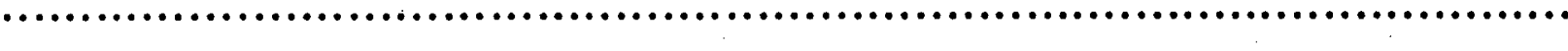

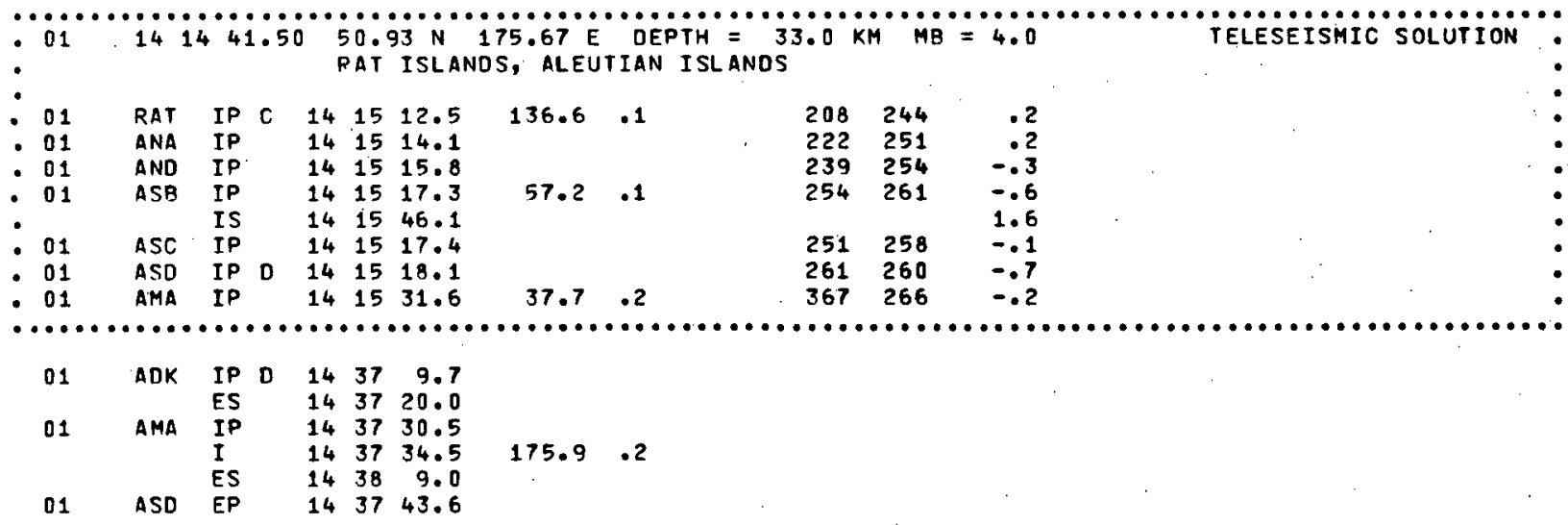




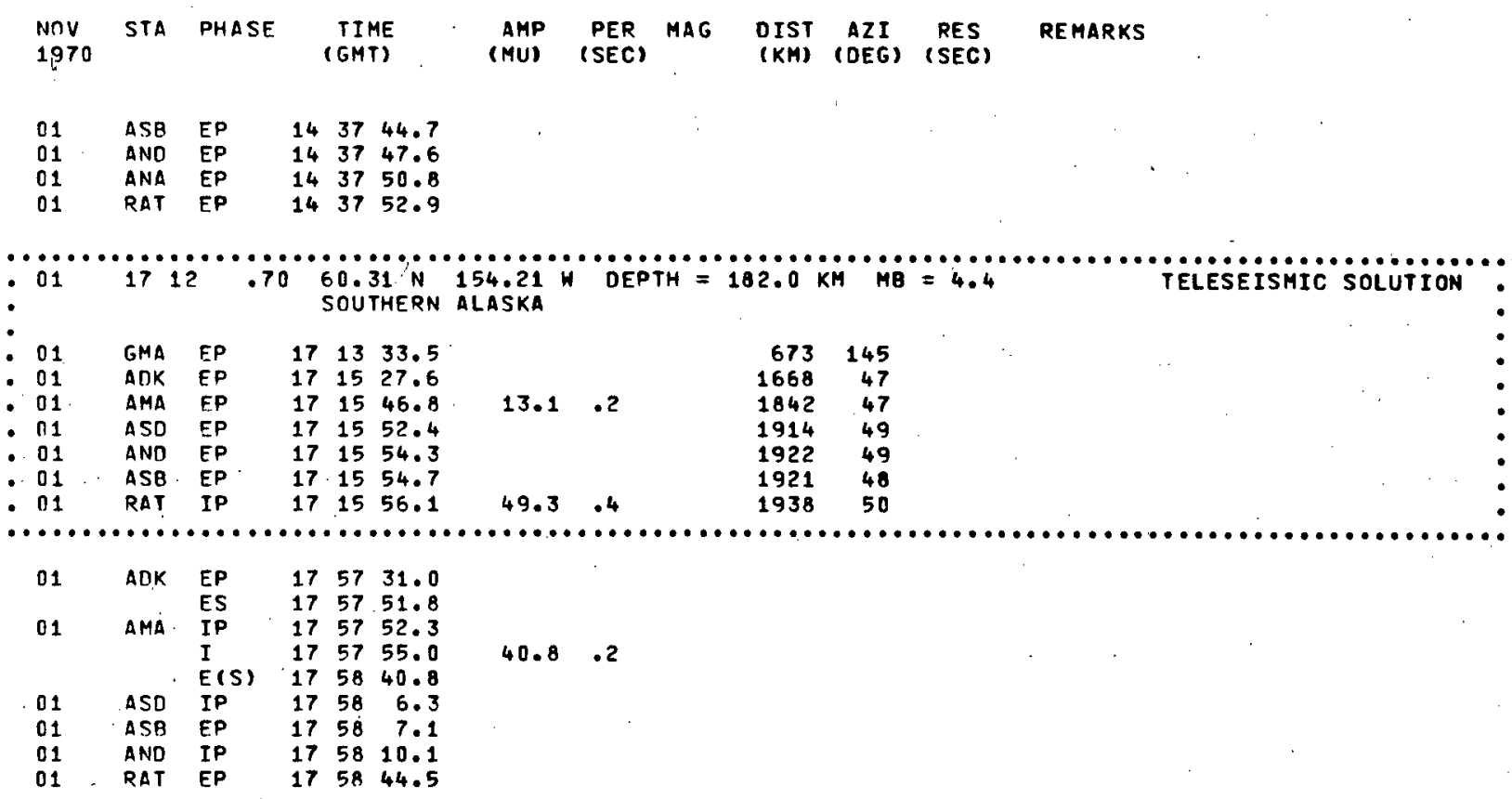

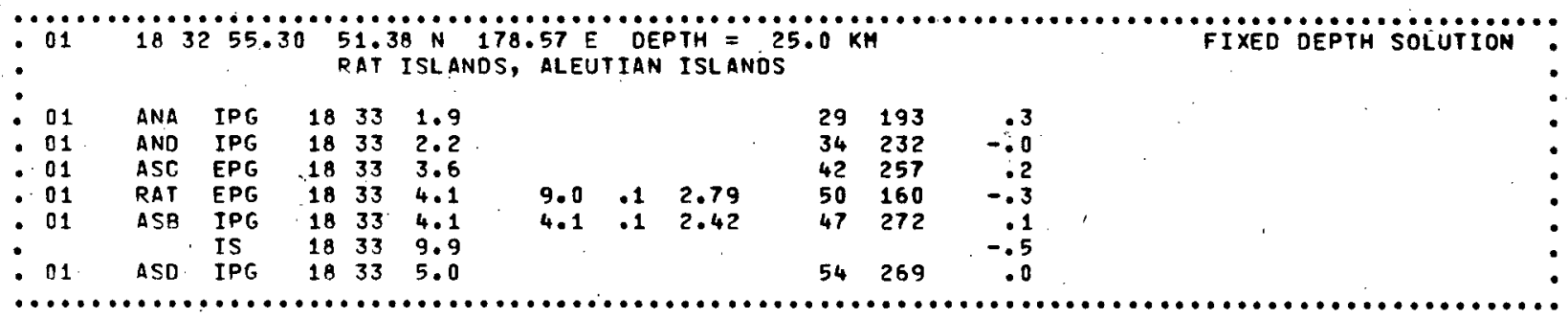

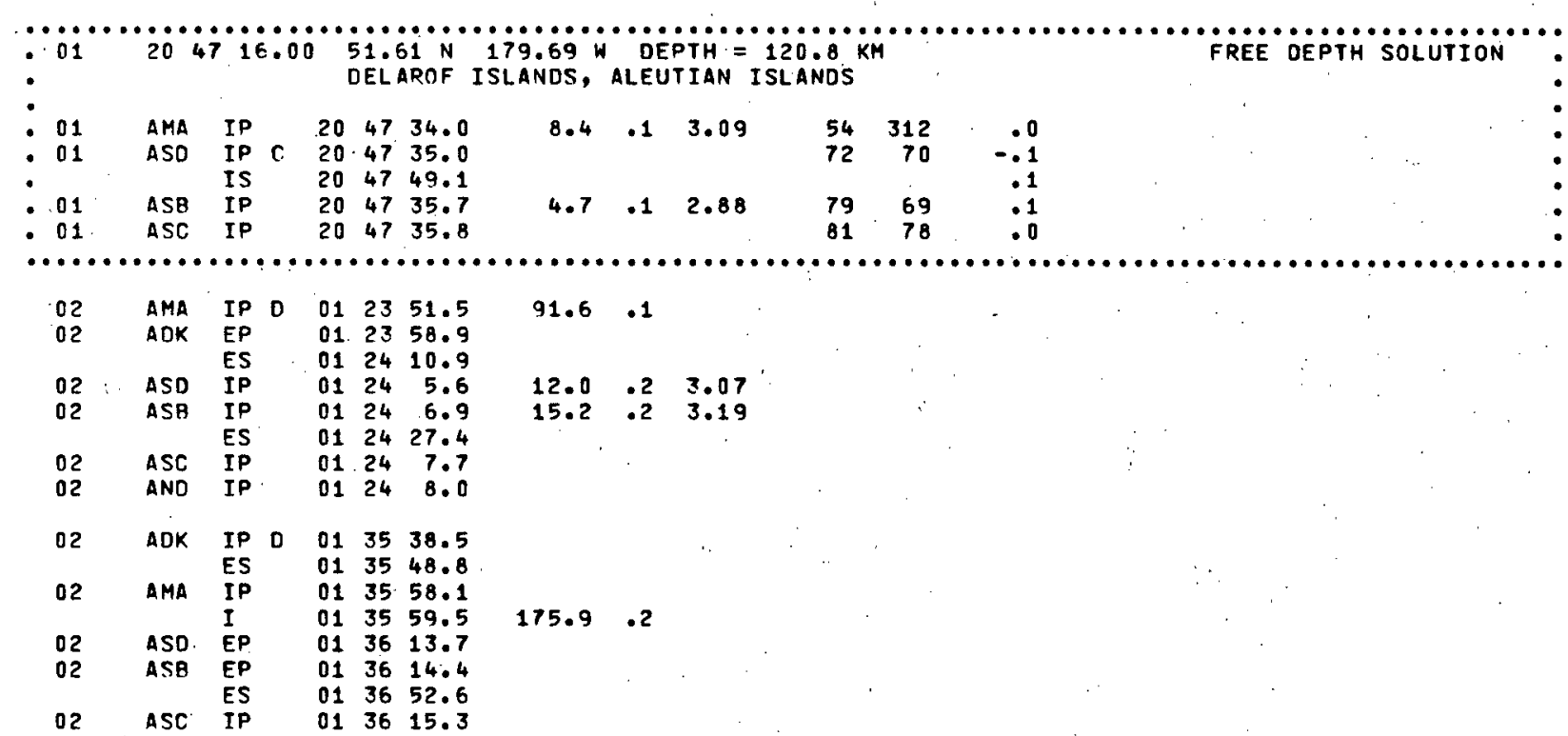




\begin{tabular}{|c|c|c|c|c|c|c|c|c|c|}
\hline $\begin{array}{l}\text { NOV } \\
1970\end{array}$ & STA & PHASE & $\begin{array}{l}\text { TIME } \\
\text { (GMT) }\end{array}$ & $\begin{array}{l}\text { AMP } \\
\text { (MU) }\end{array}$ & $\begin{array}{c}\text { PER } \\
\text { (SEC) }\end{array}$ & MAG & $\begin{array}{l}\text { DIST } \\
\text { (KM) }\end{array}$ & $\begin{array}{c}A Z I \\
(D E G)\end{array}$ & $\begin{array}{c}\text { RES } \\
\text { (SEC) }\end{array}$ \\
\hline
\end{tabular}

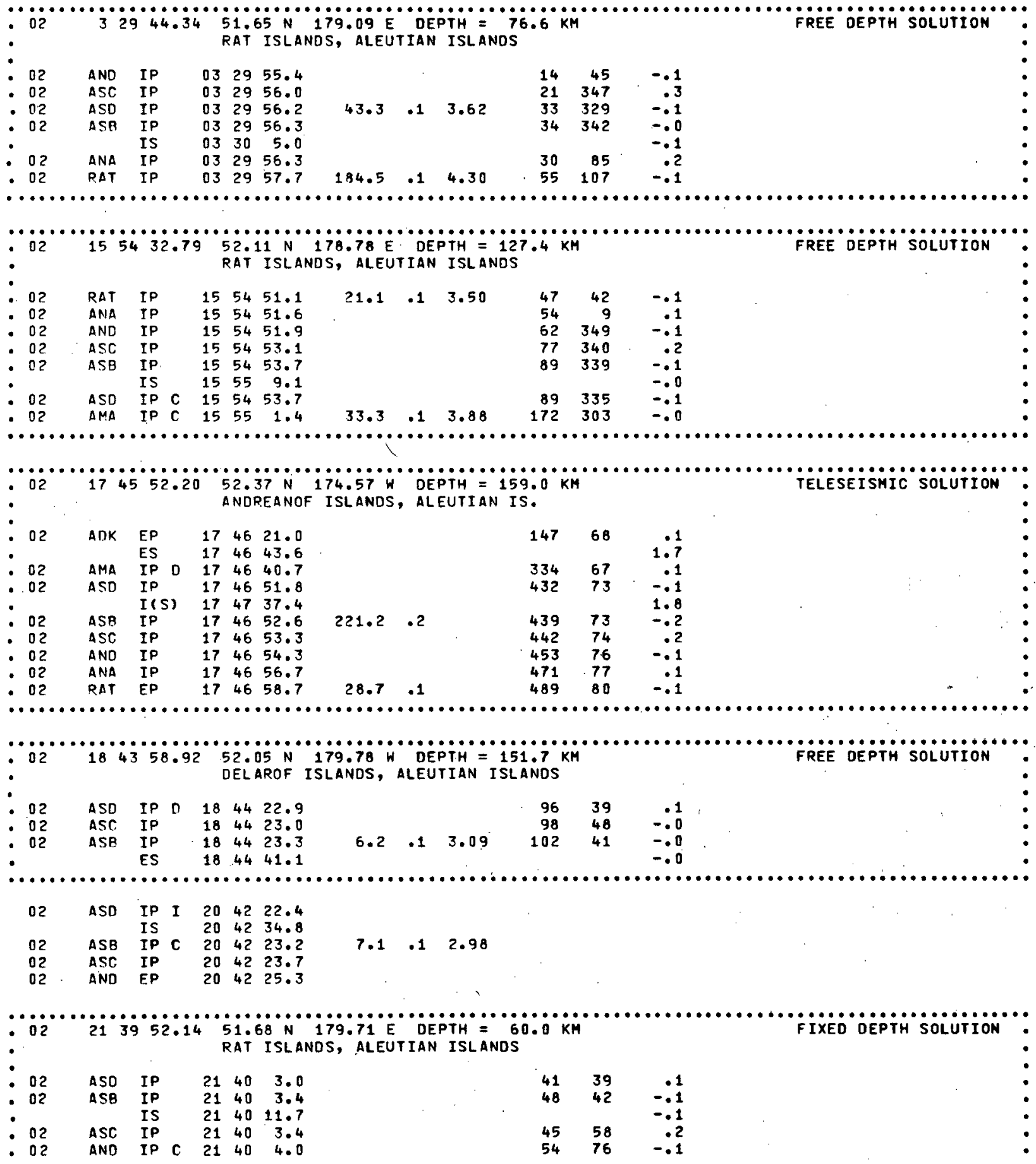




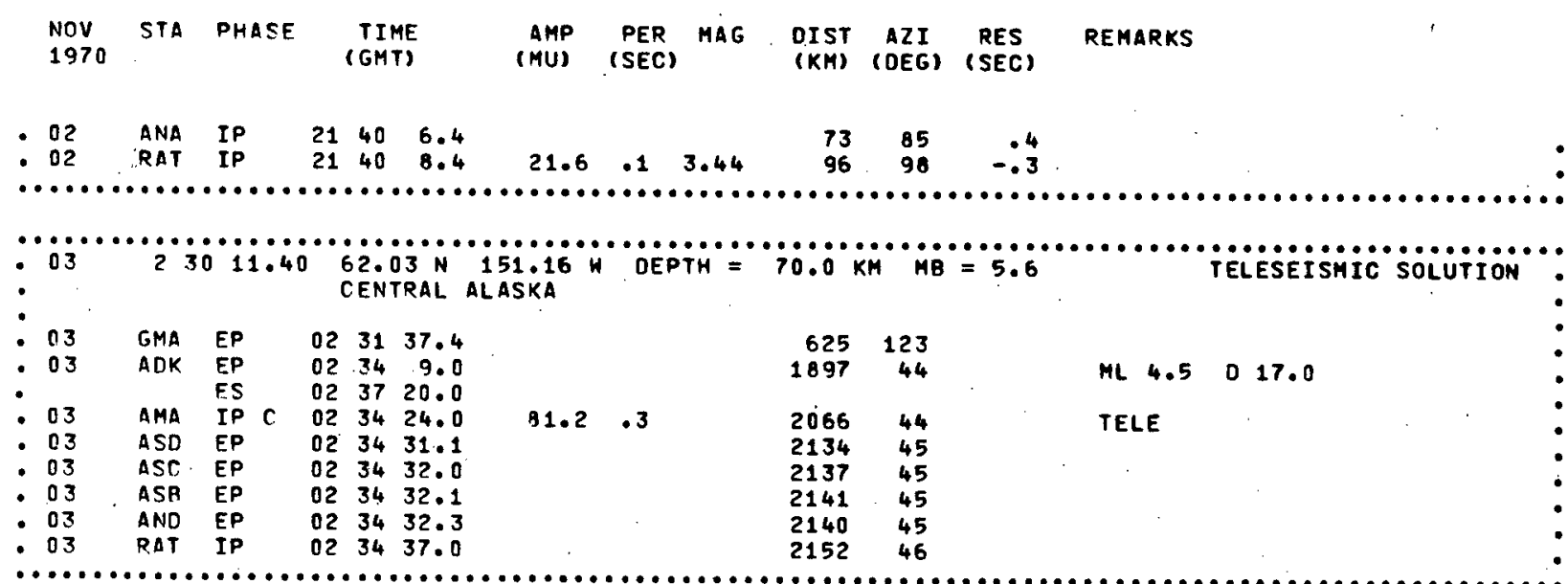

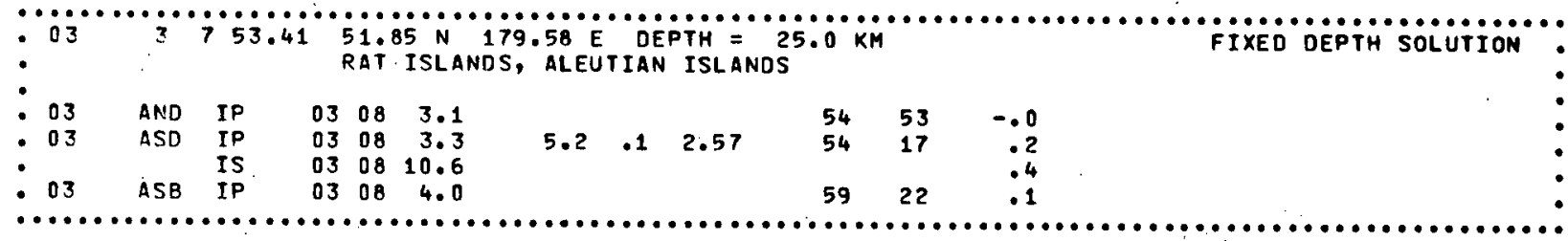

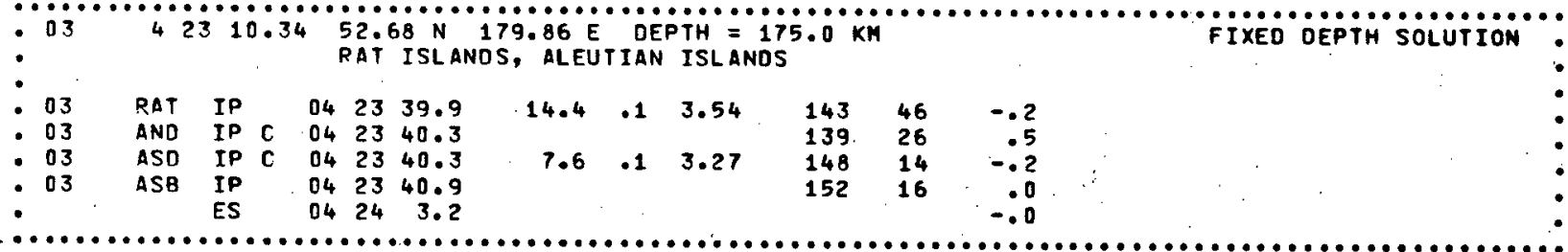

$\begin{array}{lllllllll}03 & \text { AMA } & \text { IP } & D & 06 & 39 & 16.6 & & \\ & & \text { IS } & & 06 & 39 & 21.7 & & \\ 03 & \text { ASD } & \text { IP } & C & 06 & 39 & 24.6 & & \\ 03 & \text { ASB } & \text { IP } & \text { C } & 06 & 39 & 25.3 & & \\ & & \text { IS } & & 06 & 39 & 37.2 & & \\ 03 & \text { ASC } & \text { IP } & 06 & 39 & 26.7 & & \\ 03 & \text { RAT } & \text { IP } & 06 & 39 & 35.8 & 9.6 & .1\end{array}$

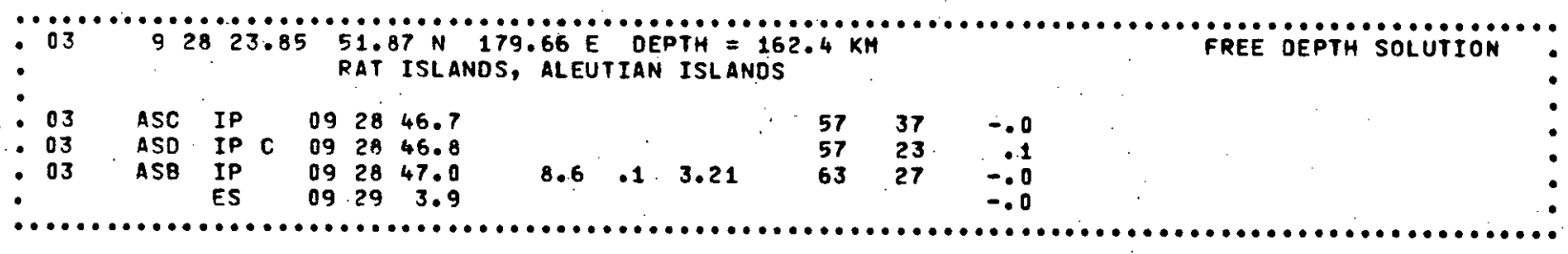

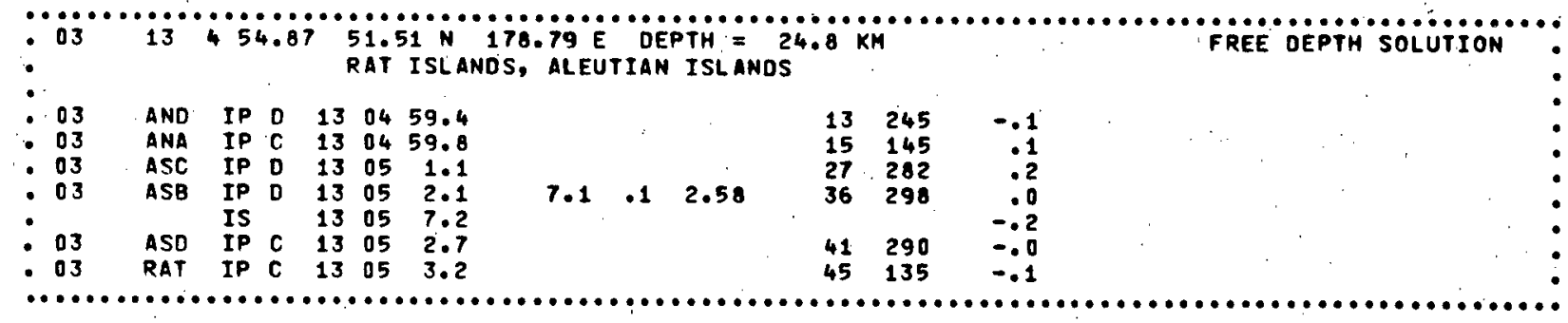




\begin{tabular}{|c|c|c|c|c|c|c|c|c|c|c|c|}
\hline $\begin{array}{l}\text { NOV } \\
1970\end{array}$ & STA & PHASE & & $\begin{aligned} \text { TIME } \\
\text { (GMT) }\end{aligned}$ & $\begin{array}{l}\text { AMP } \\
\text { (MU) }\end{array}$ & $\begin{array}{l}\text { PER } \\
\text { (SEC) }\end{array}$ & MAG & $\begin{array}{l}\text { DIST } \\
\text { (KM) }\end{array}$ & $\begin{array}{c}A Z I \\
(D E G)\end{array}$ & $\begin{array}{l}\text { RES } \\
\text { (SEC) }\end{array}$ & REMARKS : \\
\hline $\begin{array}{l}03 \\
03\end{array}$ & $\begin{array}{l}\text { ASO } \\
\text { ASB }\end{array}$ & $\begin{array}{l}\text { IP } C \\
\text { IP } \\
\text { ES } \\
\text { ID }\end{array}$ & $\begin{array}{l}14 \\
14 \\
14\end{array}$ & $\begin{array}{ll}15 & 31.6 \\
15 & 32.2 \\
15 & 40.5\end{array}$ & $\begin{array}{l}8.8 \\
5.0\end{array}$ & $\begin{array}{l}.1 \\
.1\end{array}$ & $\begin{array}{l}2.87 \\
2.65\end{array}$ & & & & . \\
\hline
\end{tabular}

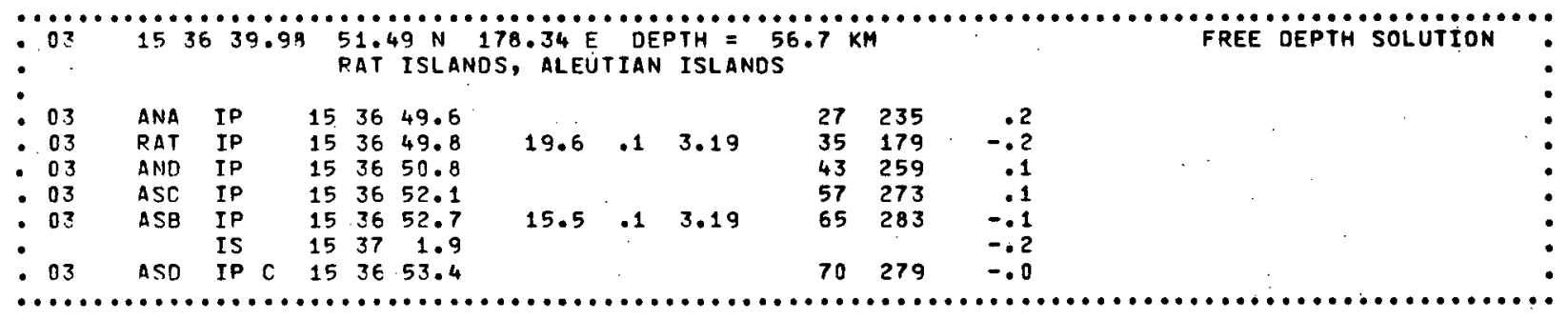

$\because 03$
03

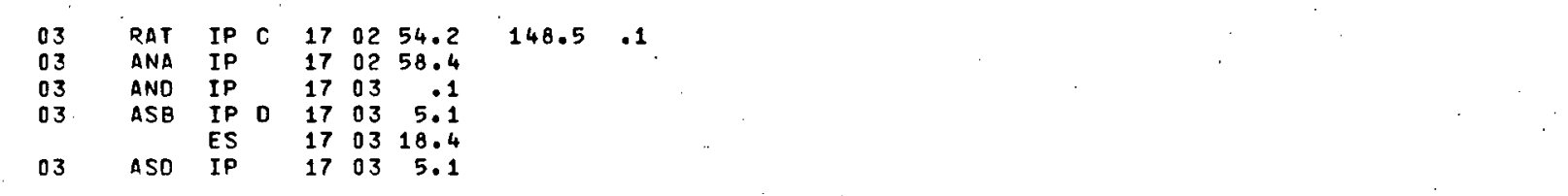

$\because 03$
$: 03$

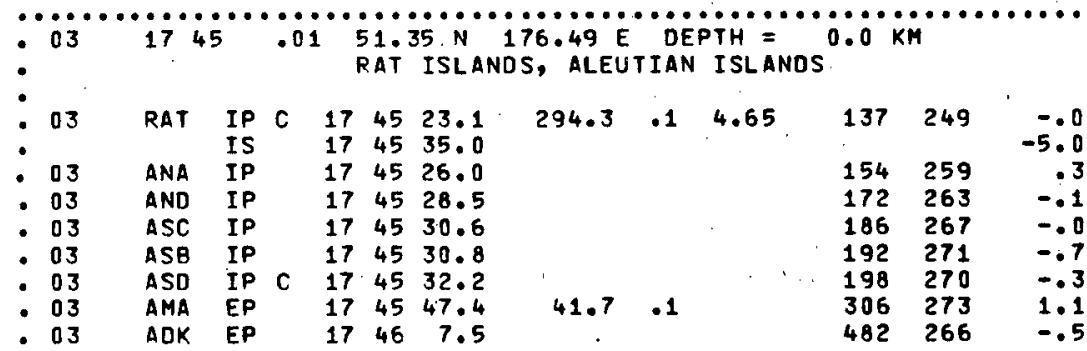




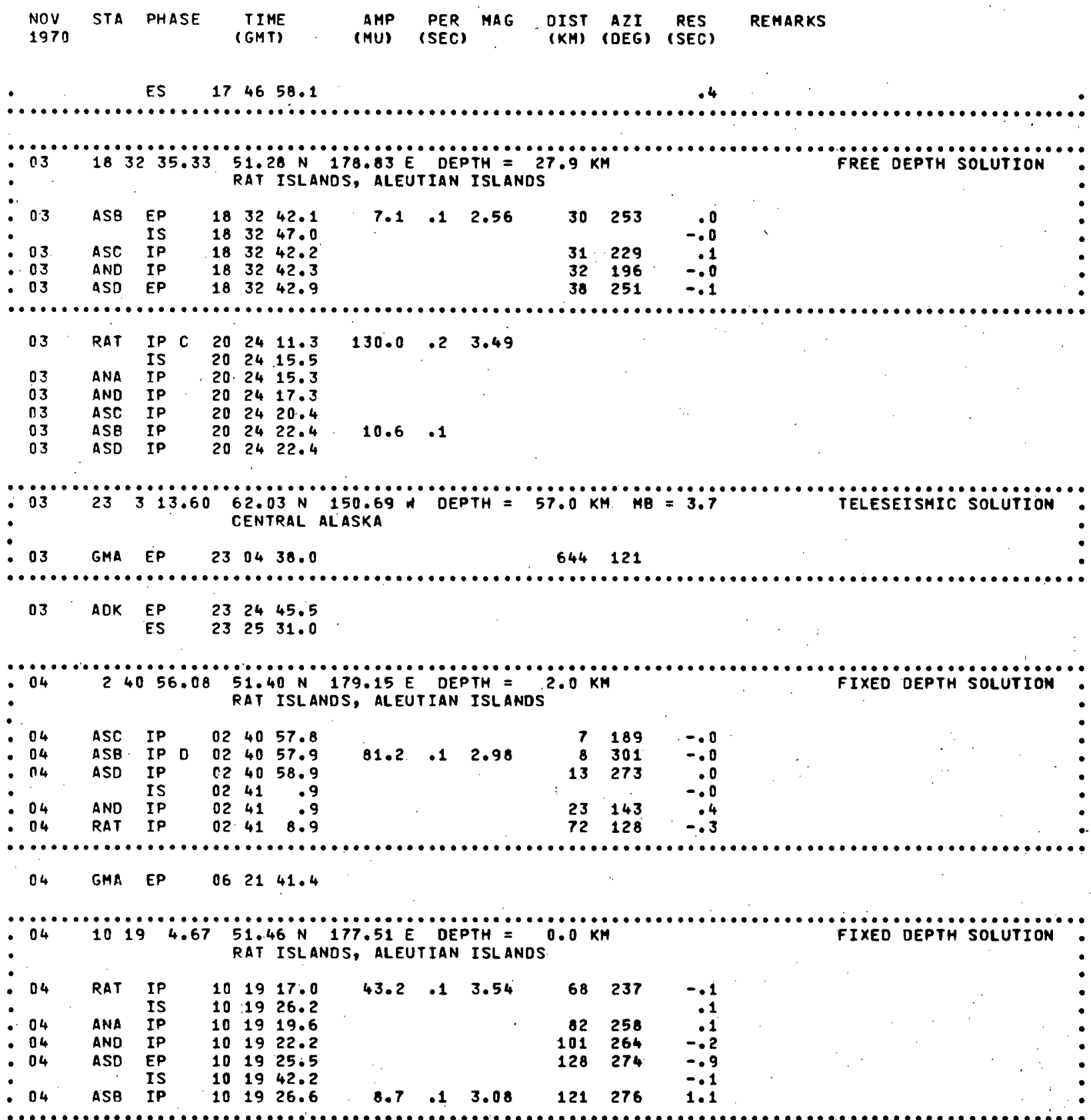

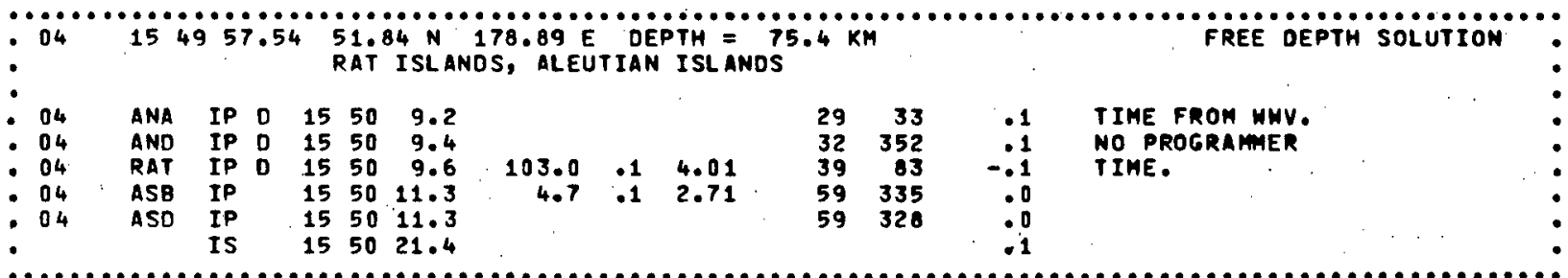




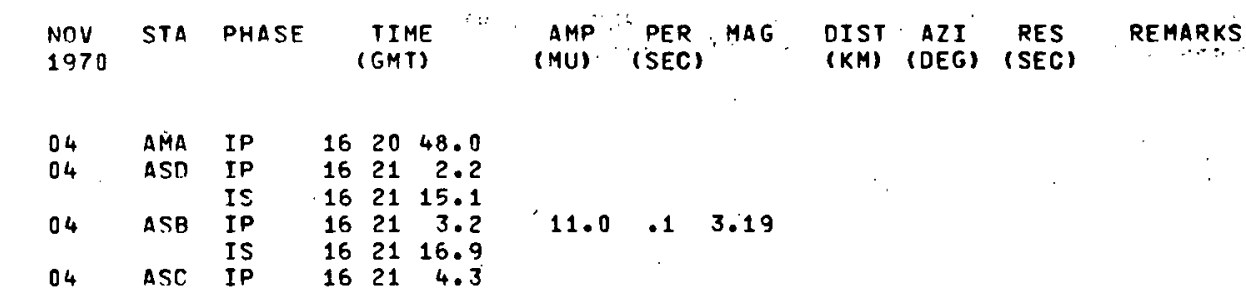

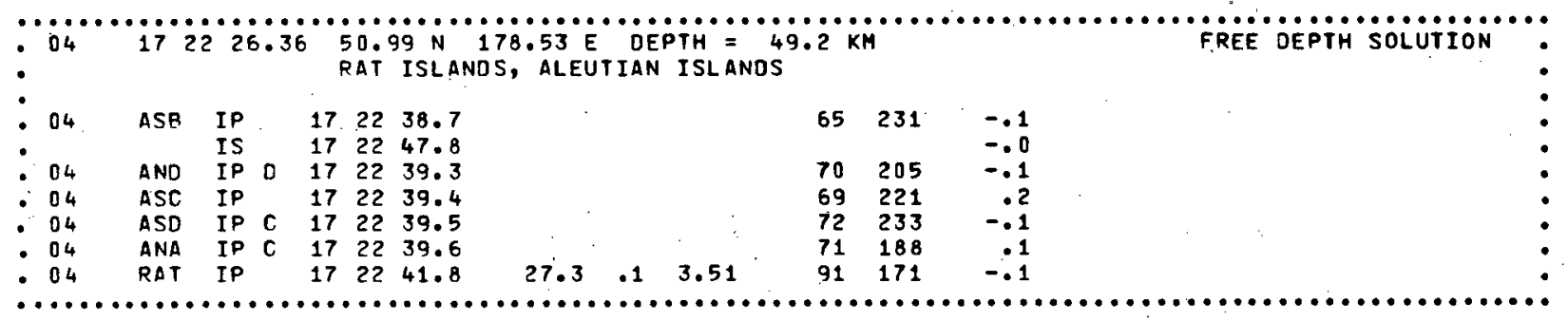

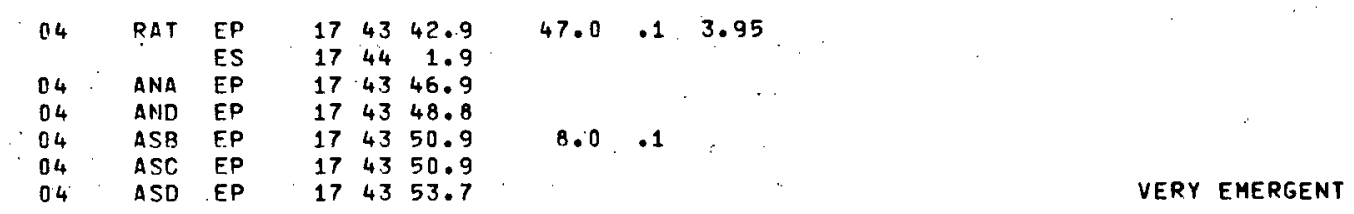

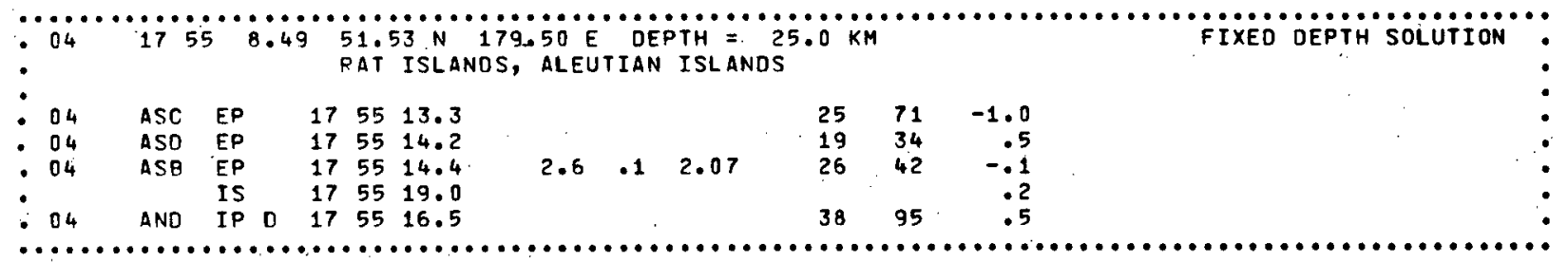

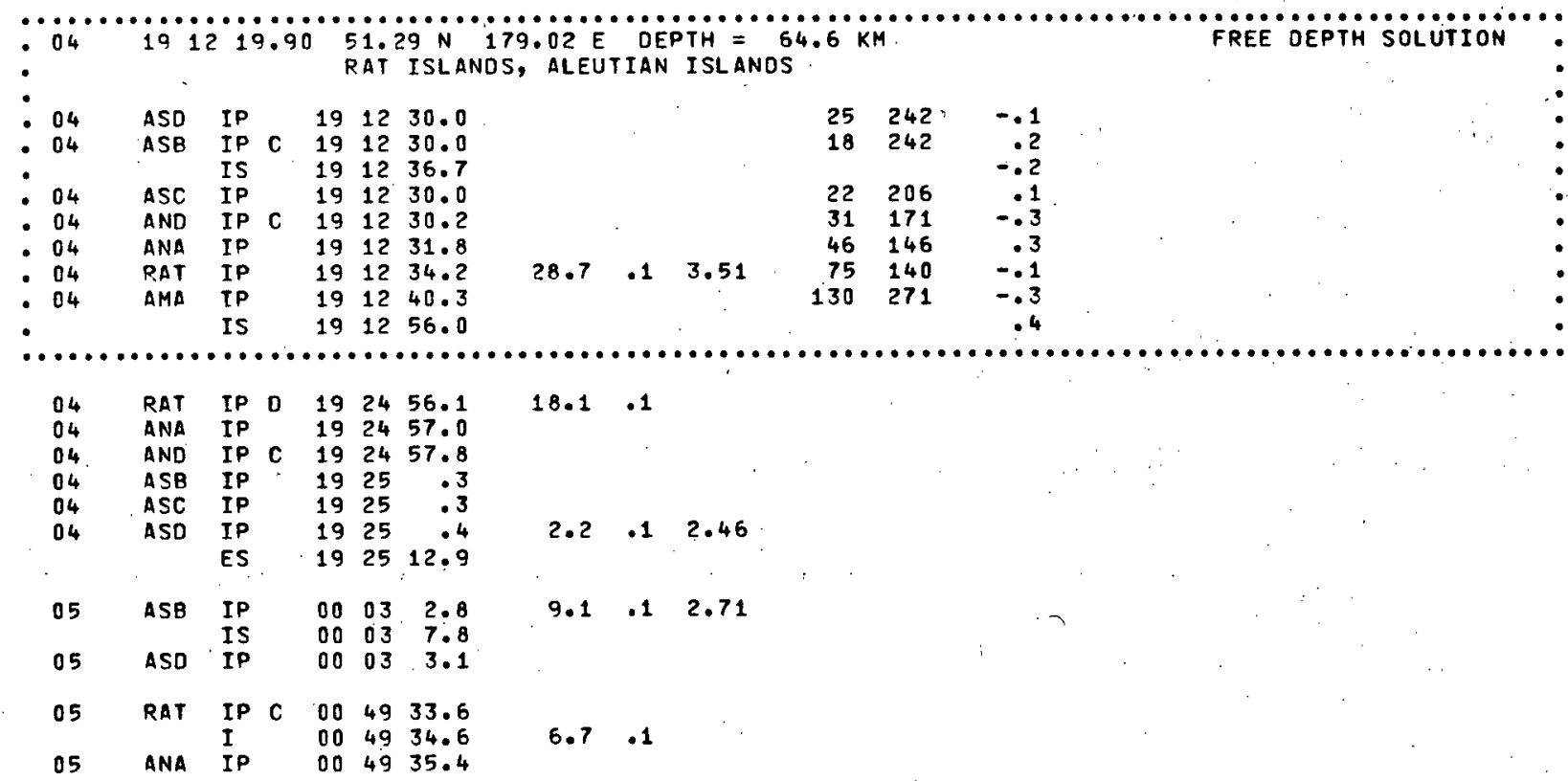




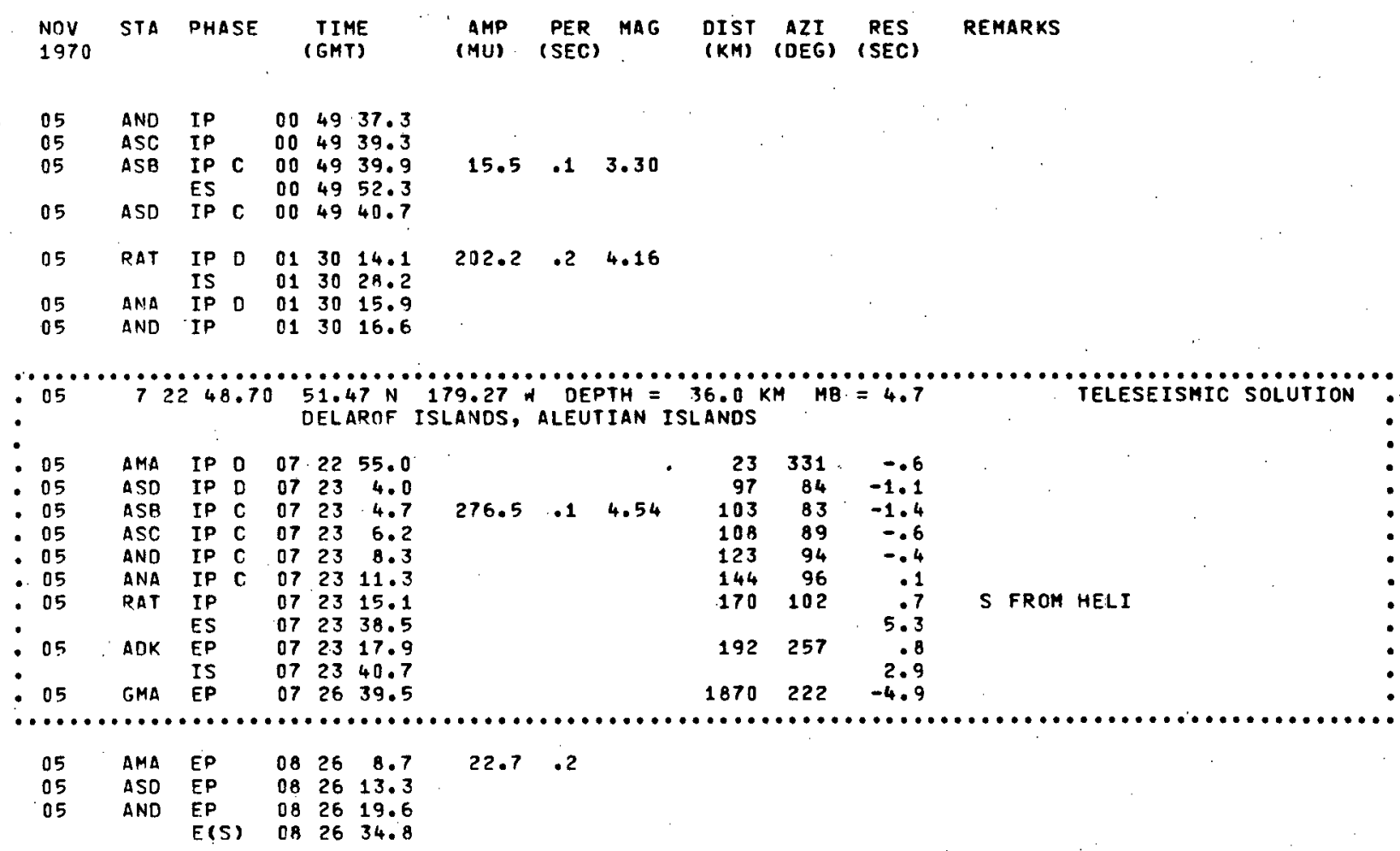

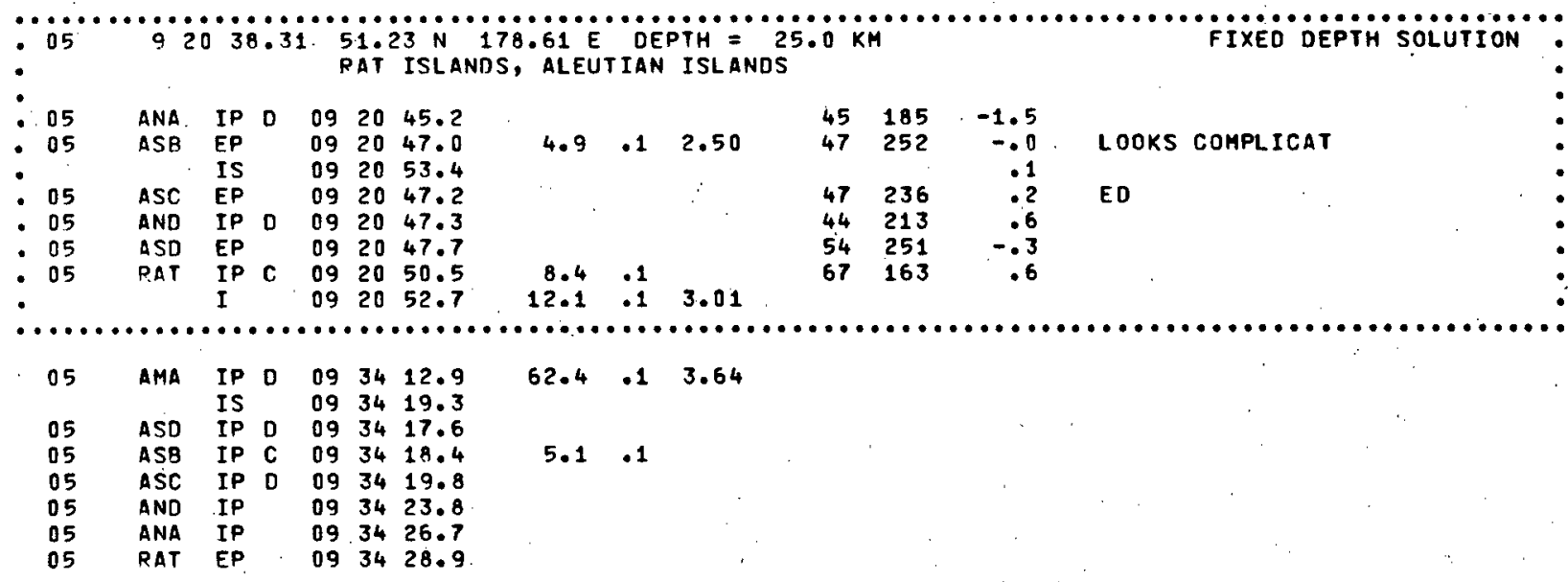

$\because 05 \quad 125311.60$ 61.67 $\mathrm{N}$ CENTRAL ALASKA $150.8 \mathrm{~W}$ DEPTH

- 05 GMA EP $125412.1 \quad 665124$

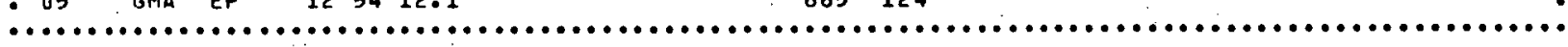

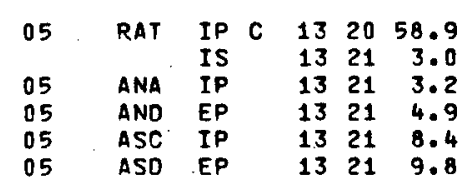

S FROM HELI. 


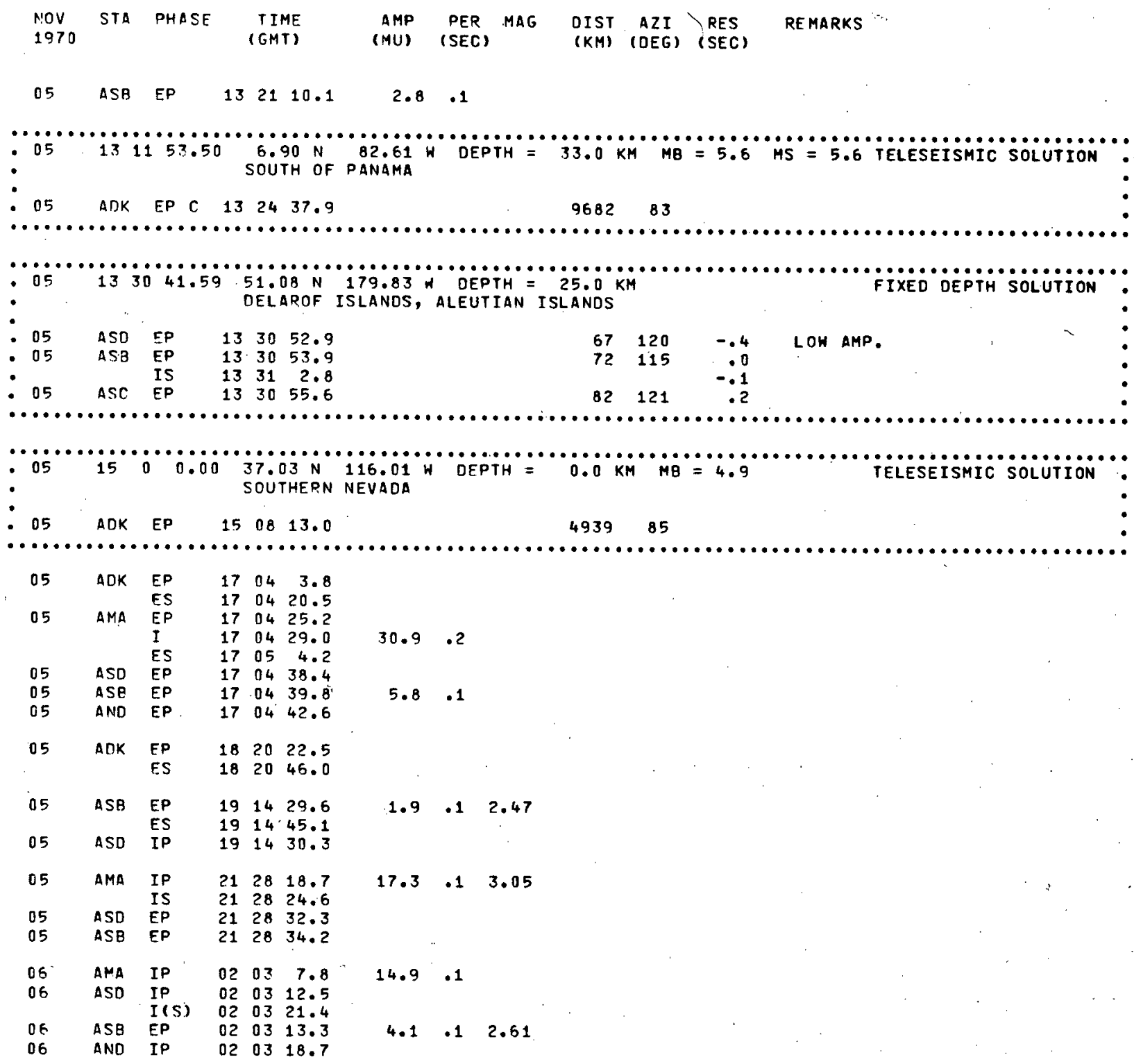

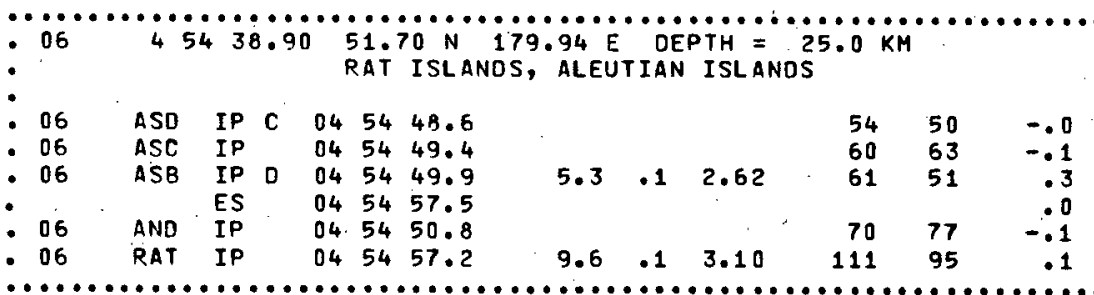




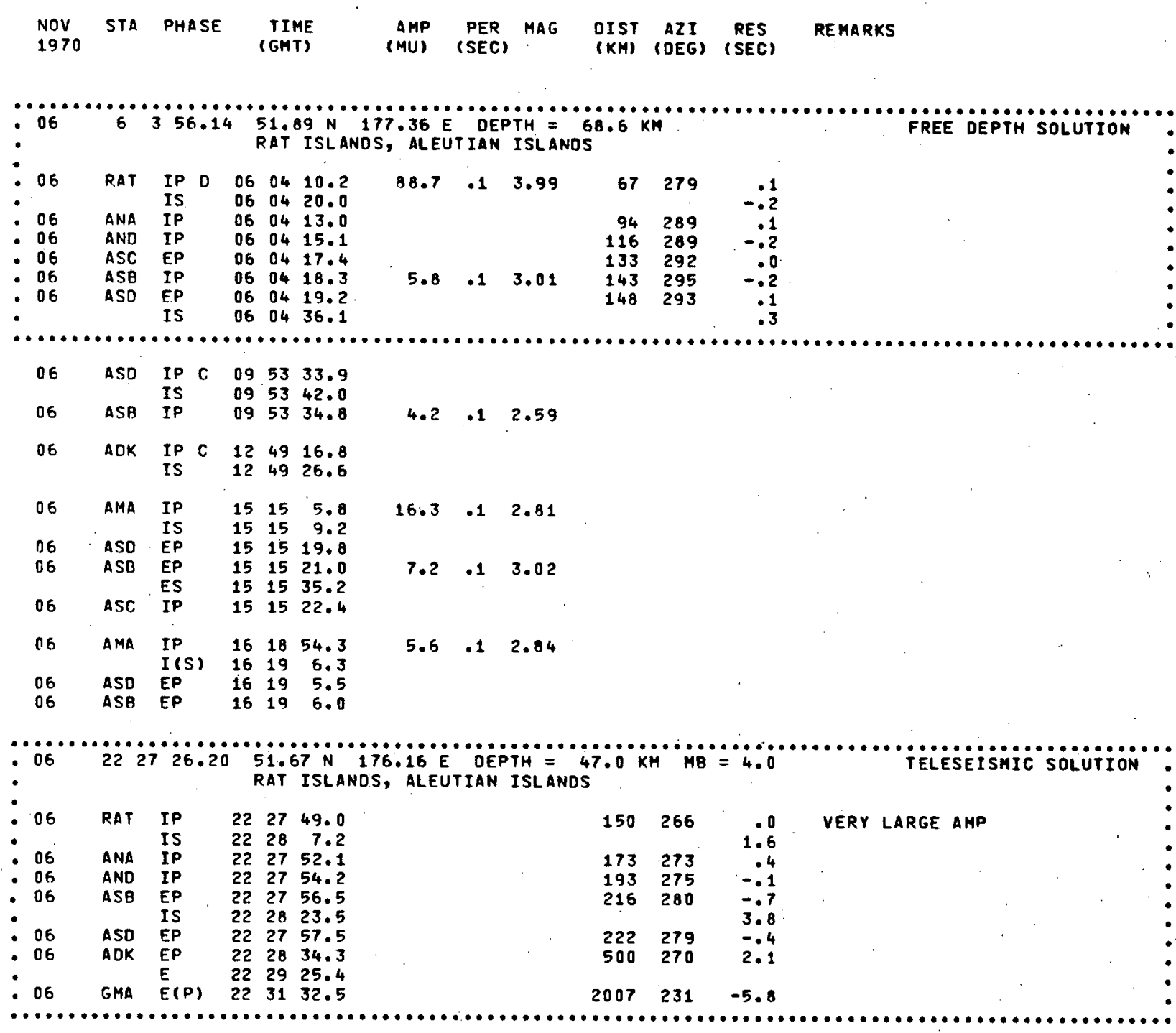

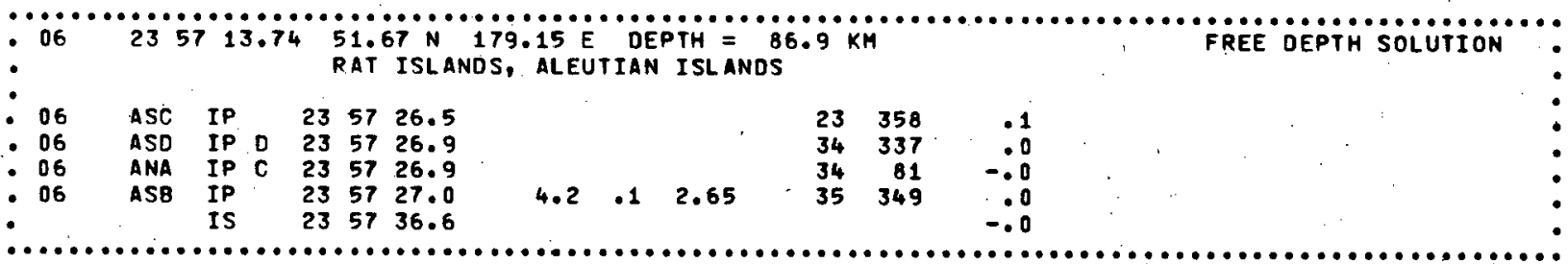

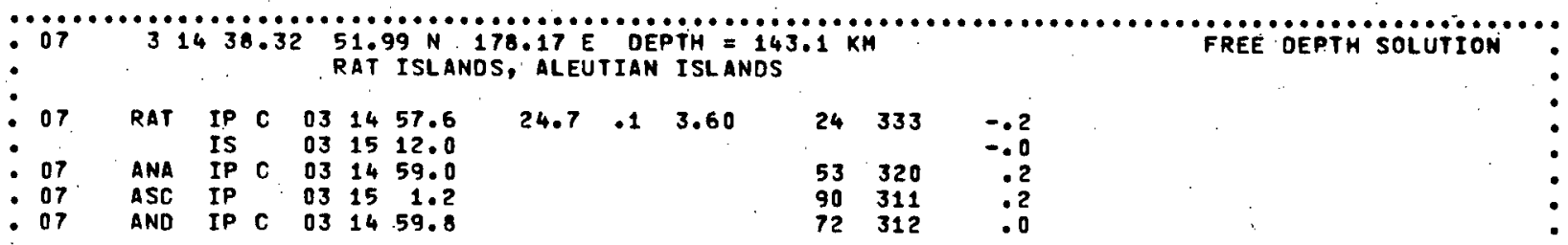




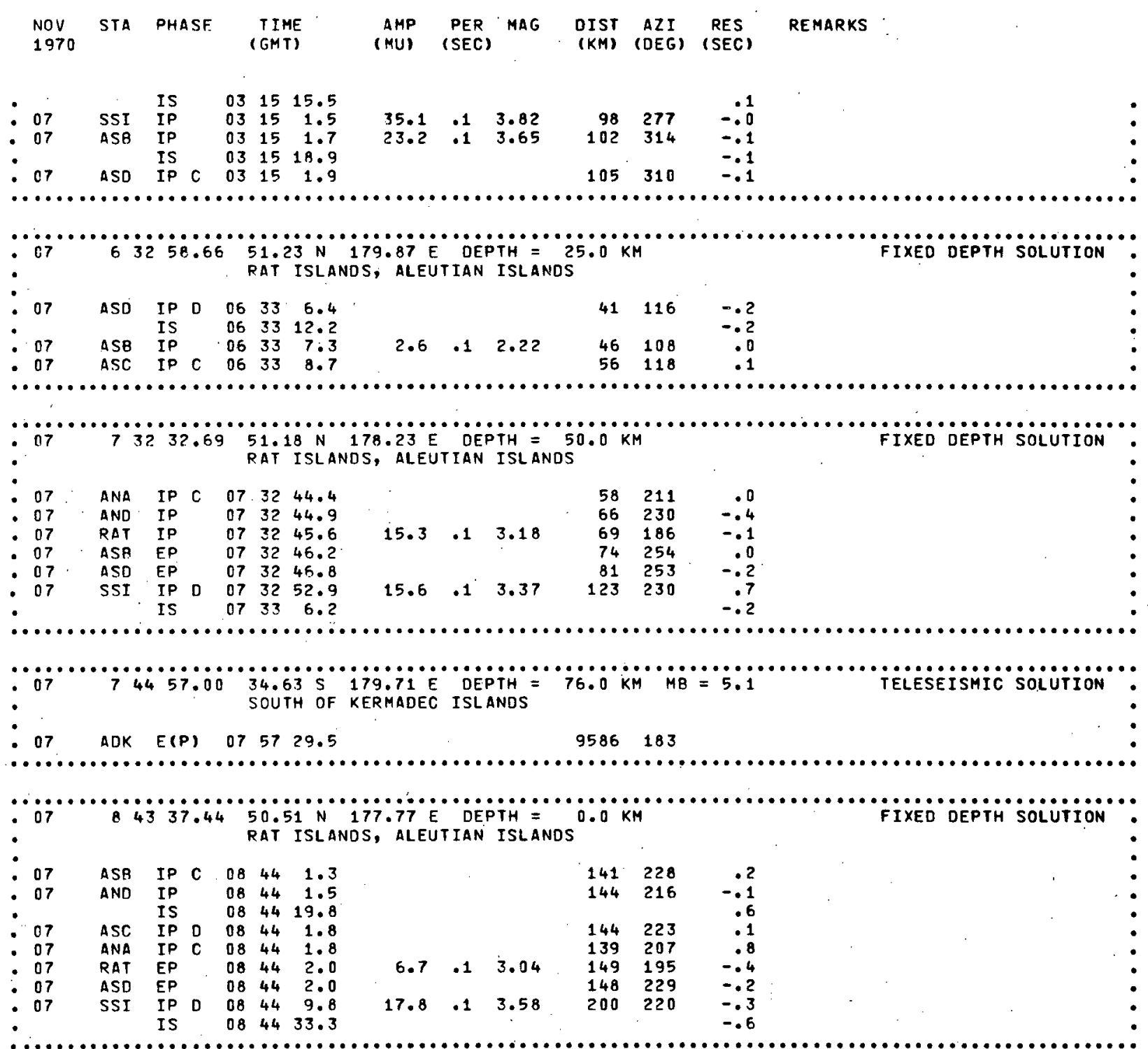

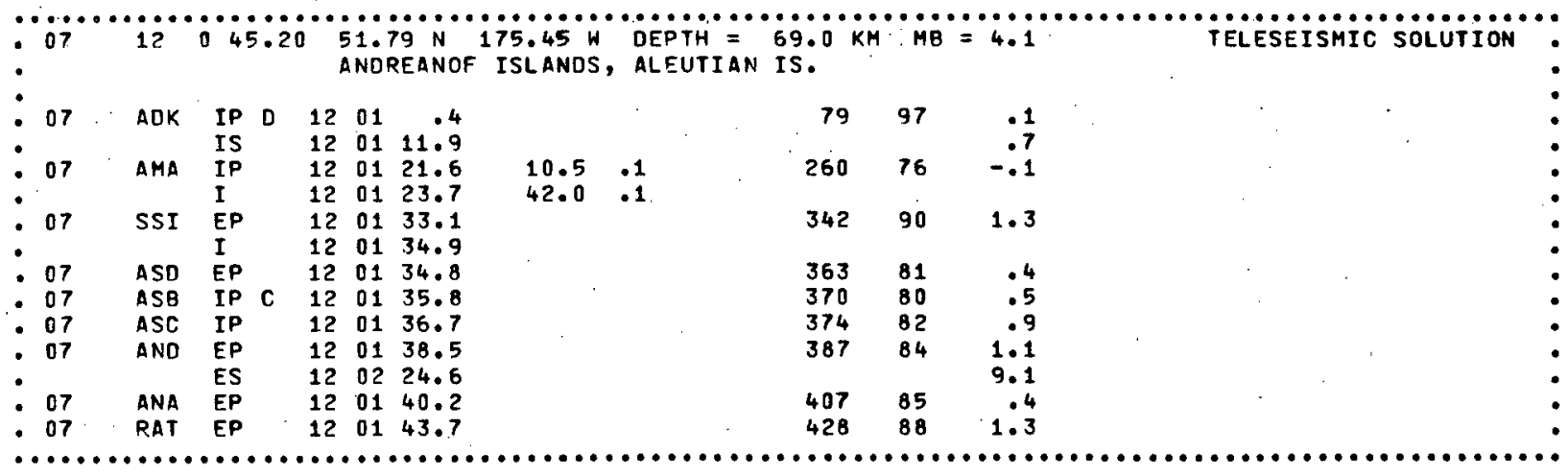




\begin{tabular}{|c|c|c|c|c|c|c|c|c|c|}
\hline $\begin{array}{l}\text { NOV } \\
1970\end{array}$ & ST & PHASE & $\begin{array}{l}\text { TIME } \\
\text { (GMT) }\end{array}$ & $\begin{array}{c}\text { AMP } \\
\text { (MU) }\end{array}$ & $\begin{array}{c}\text { PER } \\
\text { (SEC) }\end{array}$ & MAG & $\begin{array}{l}\text { DIST } \\
\text { (KM) }\end{array}$ & $\begin{array}{c}A Z I \\
(D E G)\end{array}$ & $\begin{array}{l}\text { RES } \\
\text { (SEC) }\end{array}$ \\
\hline
\end{tabular}

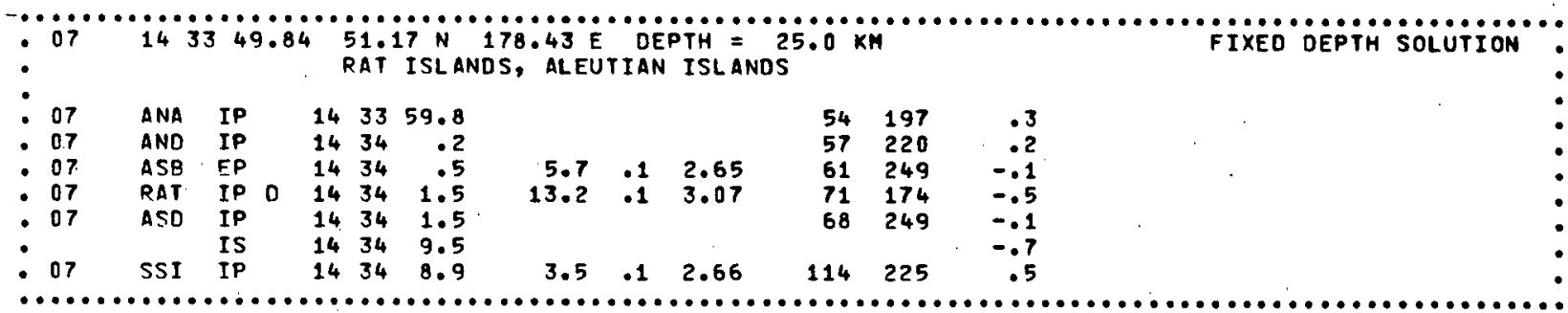

$\because 07$
$\vdots 075$
07

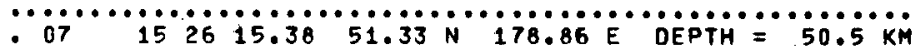

- RAT ISLANDS, ALEUTIAN ISLANDS

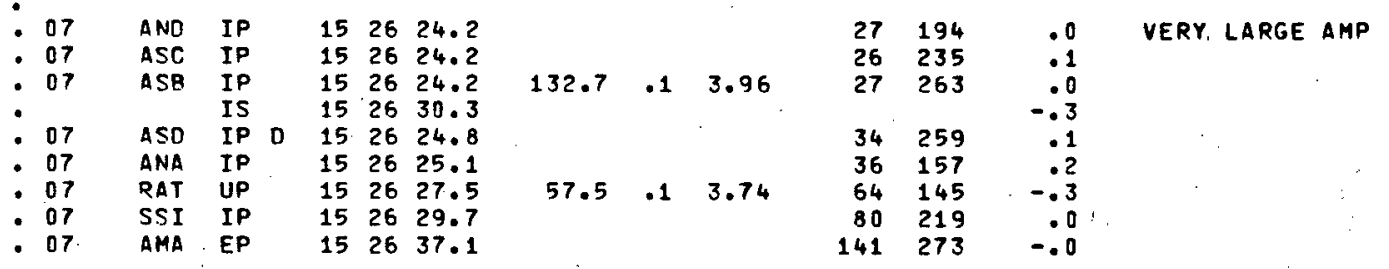

................................................................................

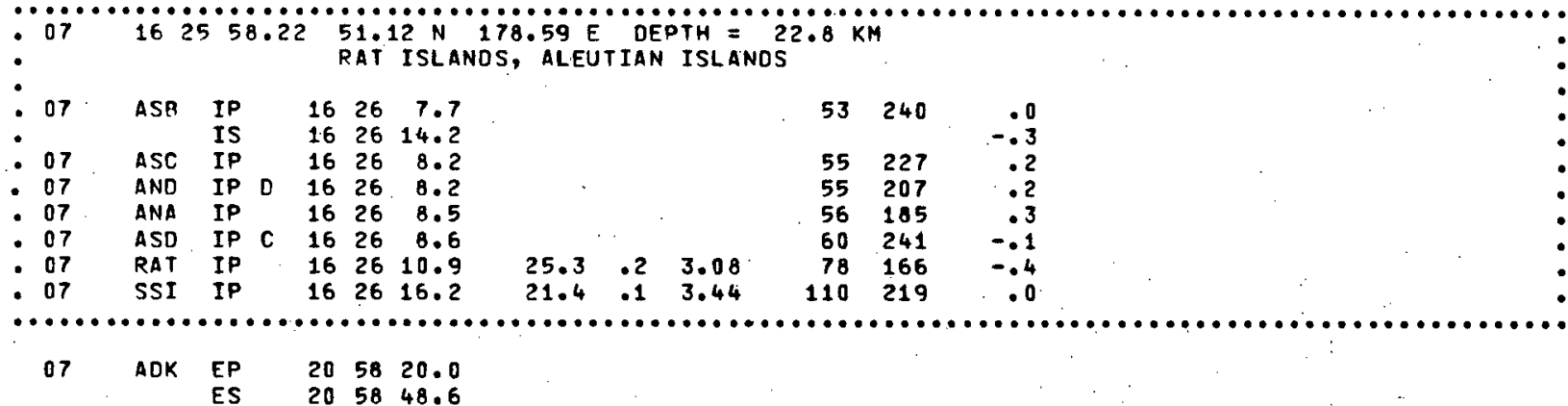

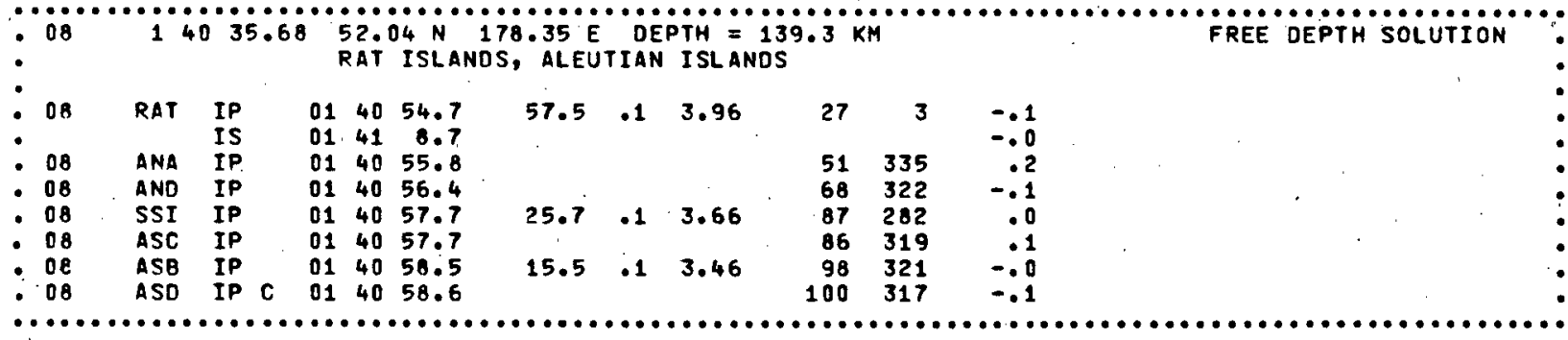




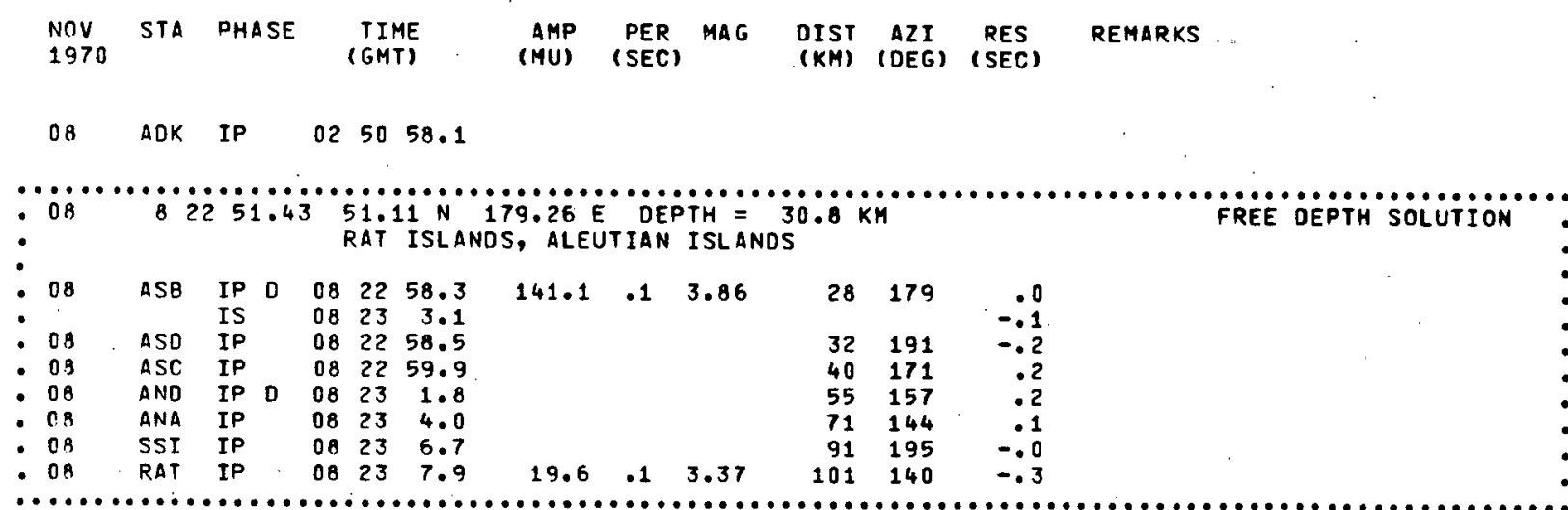

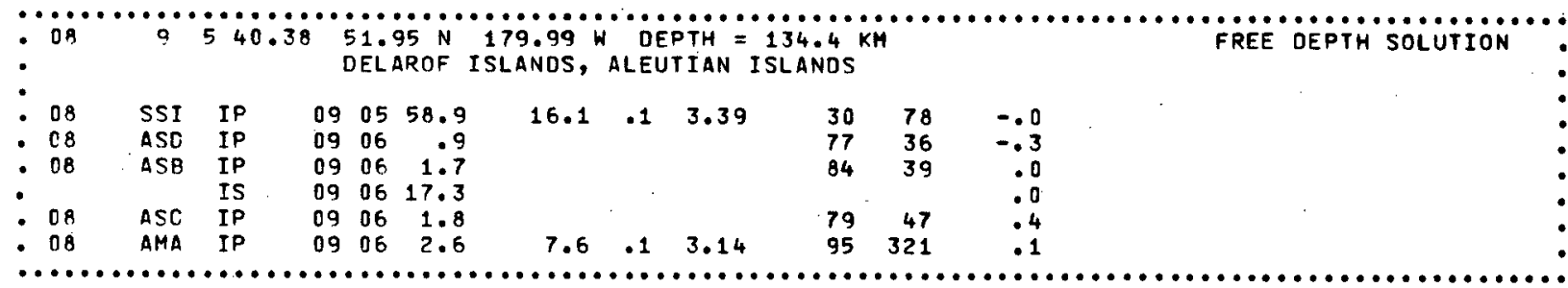

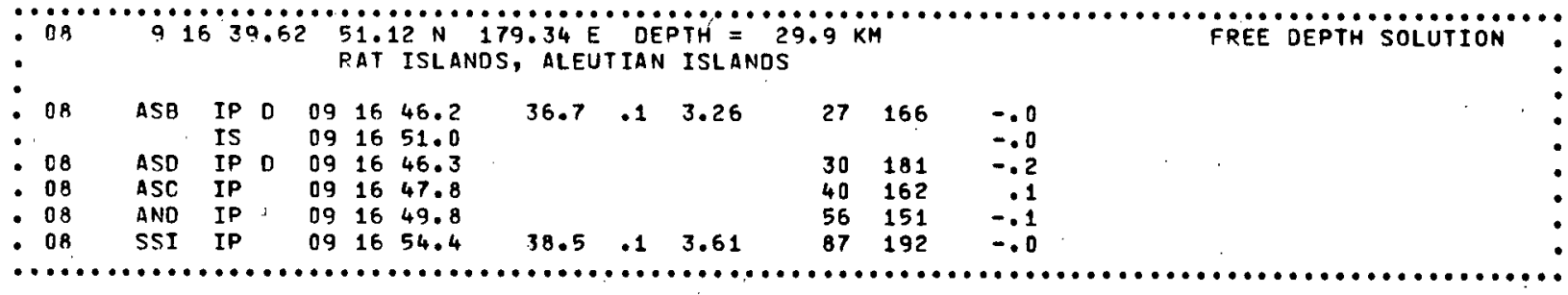

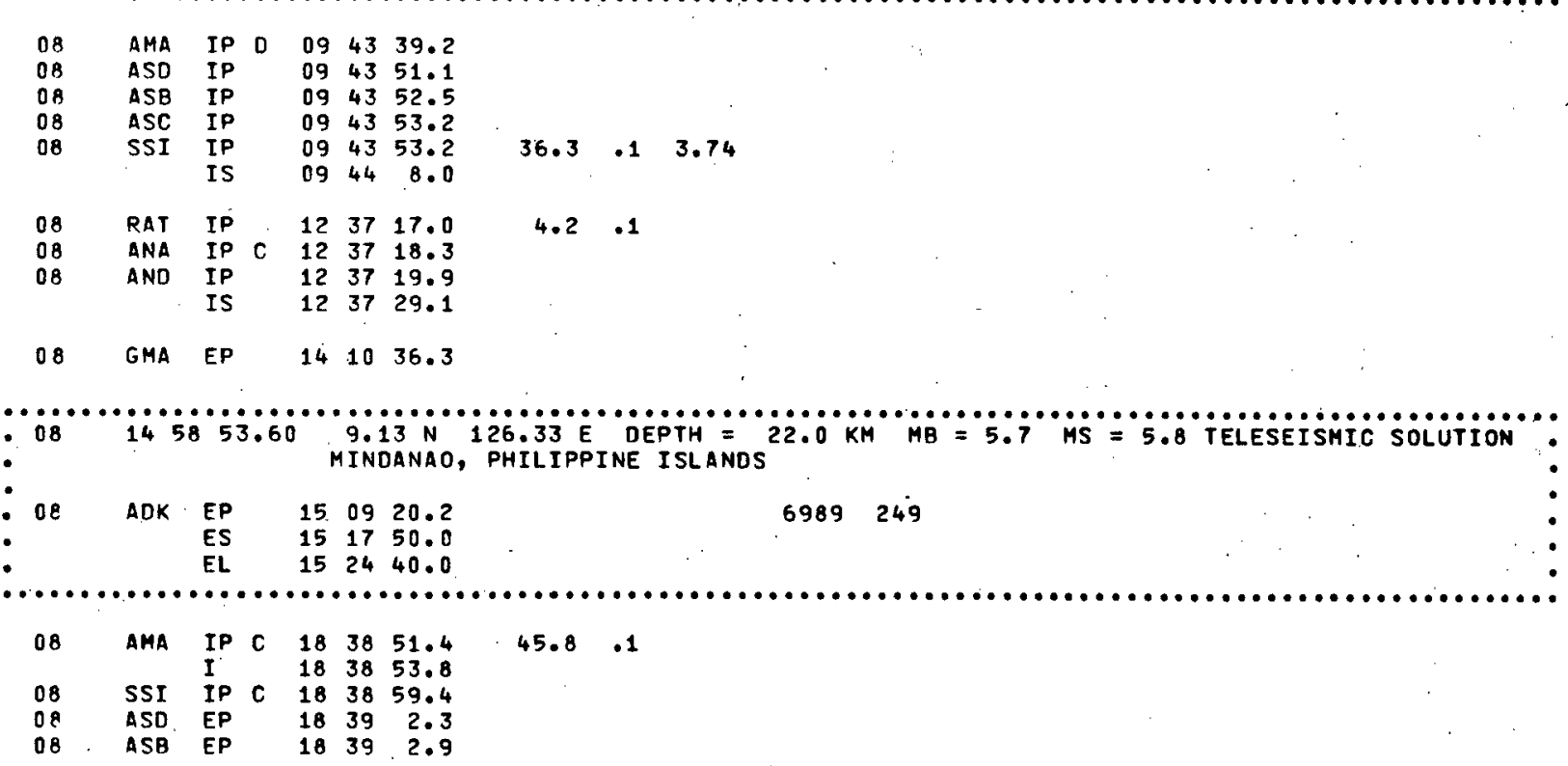




\begin{tabular}{|c|c|c|c|c|c|c|c|}
\hline $\begin{array}{l}\text { NOV } \\
1970\end{array}$ & STA & PHASE & & $\begin{array}{l}\text { TIM } \\
\text { IGMT }\end{array}$ & & $\begin{array}{l}\text { AMP } \\
\text { (MU) }\end{array}$ & $\begin{array}{l}\text { PER } \\
\text { ISEC }\end{array}$ \\
\hline 08 & $A O K$ & $\begin{array}{l}E P \\
\text { ES }\end{array}$ & $\begin{array}{l}20 \\
20\end{array}$ & $\begin{array}{l}16 \\
17\end{array}$ & $\begin{array}{r}51.9 \\
6.2\end{array}$ & & \\
\hline 08 & AMA & $\begin{array}{l}\text { IP C } \\
\text { ES }\end{array}$ & $\begin{array}{l}20 . \\
20\end{array}$ & & $\begin{array}{r}8.5 \\
32.9\end{array}$ & 38.8 & .1 \\
\hline $\begin{array}{l}08 \\
08\end{array}$ & $\begin{array}{l}\text { ASO } \\
\text { ASB }\end{array}$ & $\begin{array}{l}\text { IP } \\
\text { IP }\end{array}$ & $\begin{array}{l}20 \\
20\end{array}$ & $\begin{array}{l}17 \\
17\end{array}$ & $\begin{array}{l}19.8 \\
20.5\end{array}$ & 8.2 & .1 \\
\hline $\begin{array}{l}08 \\
08\end{array}$ & $\begin{array}{l}\text { RAT } \\
\text { ANA }\end{array}$ & $\begin{array}{l}\text { IP } \\
\text { IP } \\
\text { IS }\end{array}$ & $\begin{array}{l}21 \\
21 \\
21\end{array}$ & $\begin{array}{l}52 \\
52 \\
53\end{array}$ & $\begin{array}{r}58.3 \\
58.3 \\
6.2\end{array}$ & 6.0 & .1 \\
\hline $\begin{array}{l}08 \\
08\end{array}$ & $\begin{array}{l}\text { AND } \\
\text { SSI }\end{array}$ & $\begin{array}{l}\text { EP } \\
\text { IP } \\
\text { IS }\end{array}$ & $\begin{array}{l}21 \\
21 \\
21\end{array}$ & $\begin{array}{l}53 \\
53 \\
53\end{array}$ & $\begin{array}{r}.4 \\
8.2 \\
22.8\end{array}$ & & \\
\hline
\end{tabular}

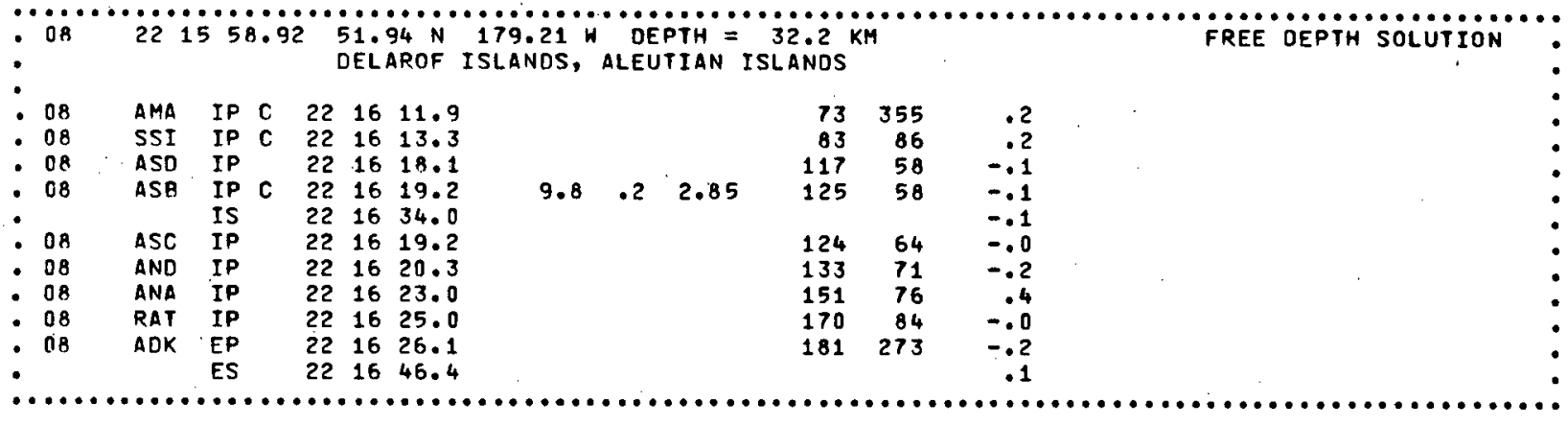

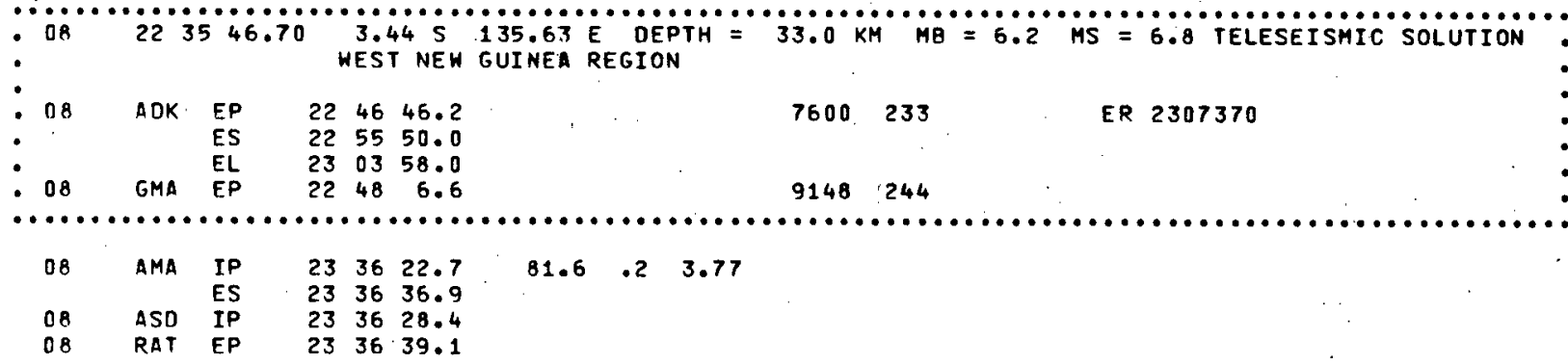

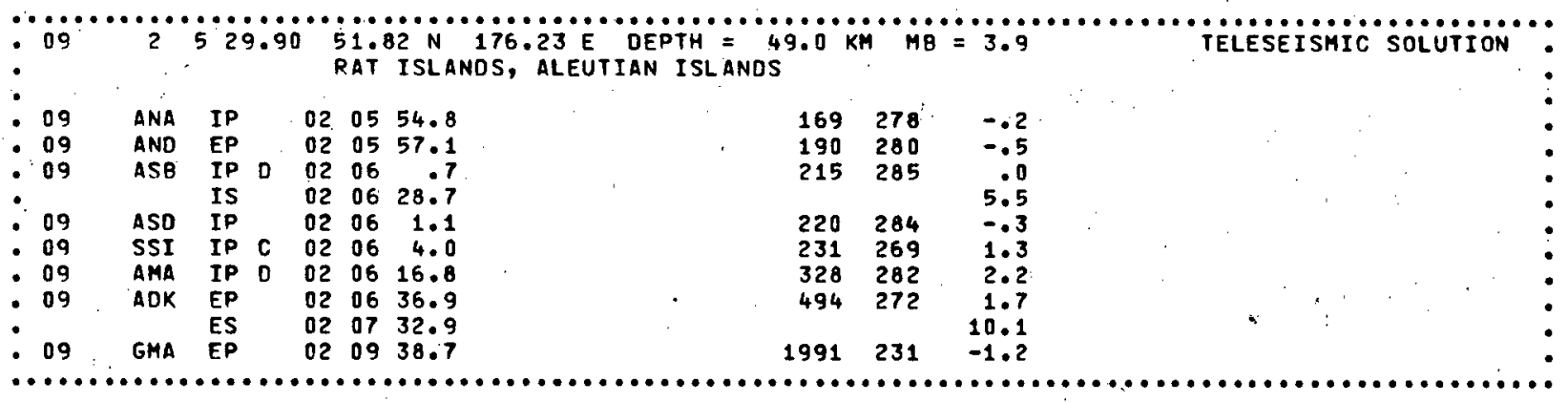

\begin{tabular}{|c|c|c|c|c|c|c|c|c|c|c|}
\hline $\begin{array}{l}09 \\
09\end{array}$ & $\begin{array}{l}\text { ASB } \\
\text { ASO }\end{array}$ & $\begin{array}{l}\text { IP } \\
\text { IS } \\
\text { IP }\end{array}$ & $\begin{array}{ll}\mathrm{C} & 02 \\
& 02 \\
0 & 02\end{array}$ & $\begin{array}{l}46 \\
46 \\
46\end{array}$ & $\begin{array}{l}2.0 \\
6.4 \\
2.4\end{array}$ & 108.2 & .1 & 3.72 & 26 & $\begin{array}{l}197 \\
206\end{array}$ \\
\hline
\end{tabular}




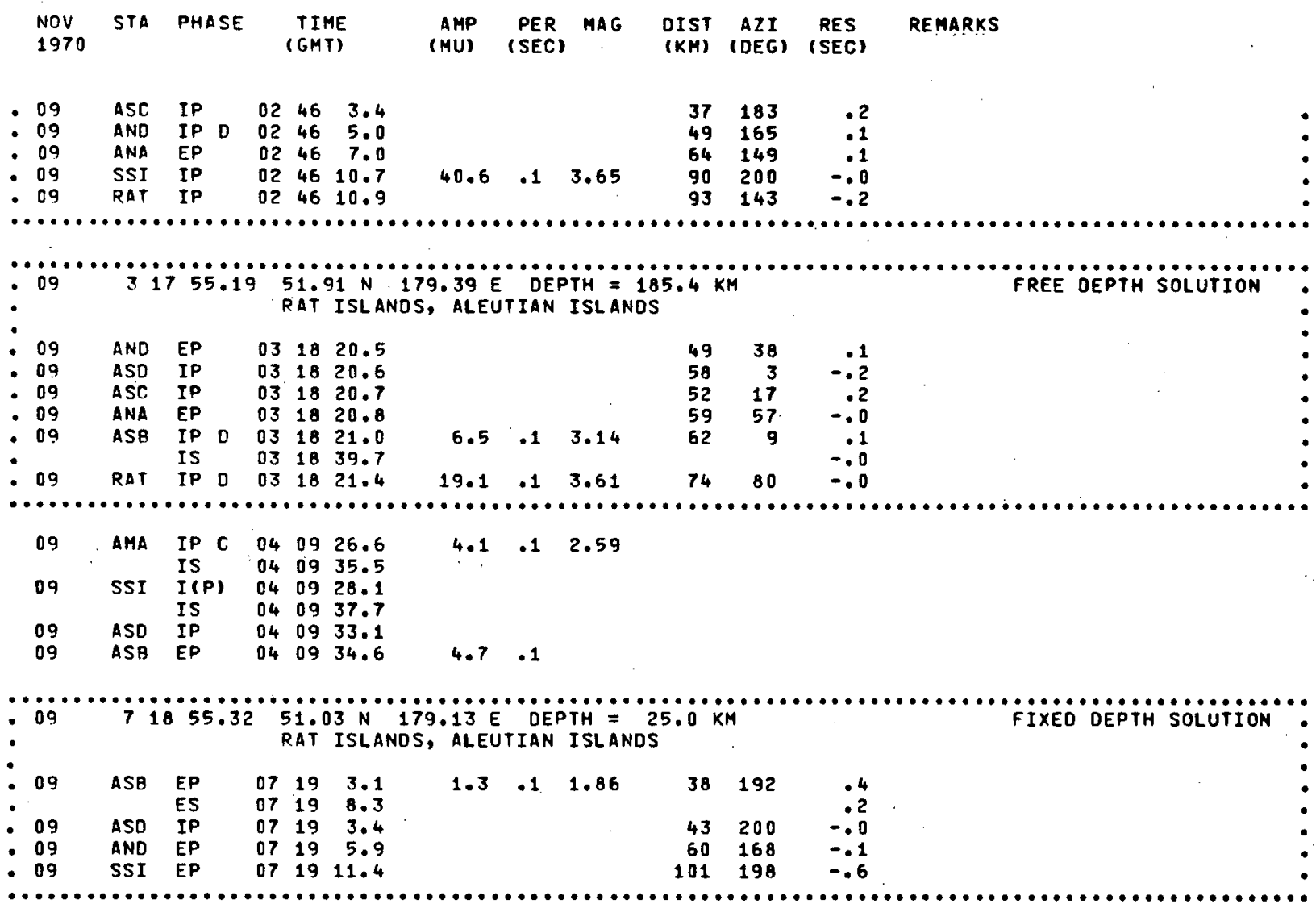

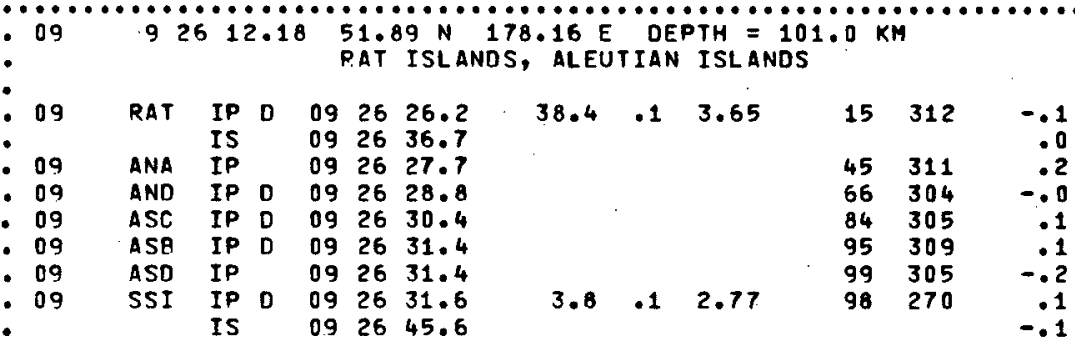

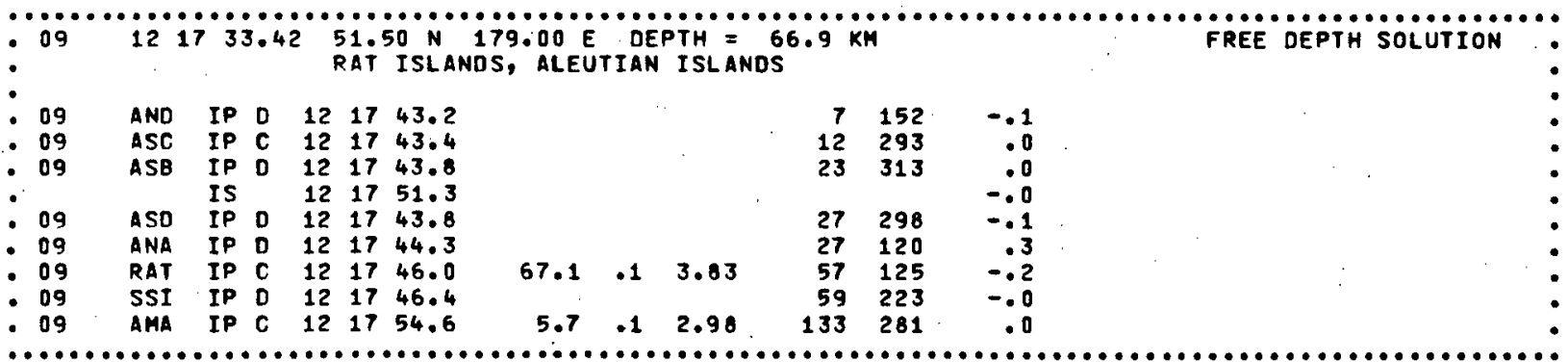




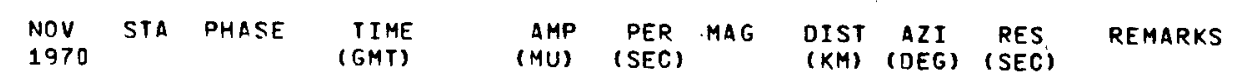

\begin{tabular}{|c|c|c|c|c|c|c|c|c|c|c|c|}
\hline $\begin{array}{l}09 \\
-\quad 09 \\
-\quad 09 \\
-\quad 09 \\
-\quad 09\end{array}$ & $\begin{array}{l}\text { AND } \\
\text { ASB } \\
\text { RAT } \\
\text { ASD } \\
\text { SSI }\end{array}$ & $\begin{array}{l}E P \\
E P \\
E P \\
E P \\
\text { EP } \\
\text { IS }\end{array}$ & $\begin{array}{l}12 \\
12 \\
12 \\
12 \\
12 \\
12\end{array}$ & $\begin{array}{l}25 \\
25 \\
25 \\
25 \\
25 \\
25\end{array}$ & $\begin{array}{l}42.9 \\
44.3 \\
44.4 \\
44.4 \\
46.1 \\
56.4\end{array}$ & $\begin{array}{l}1.5 \\
3.3 \\
2.2\end{array}$ & $\begin{array}{l}.1 \\
.1 \\
.1\end{array}$ & $\begin{array}{l}2.17 \\
2.52 \\
2.40\end{array}$ & $\begin{array}{l}10 \\
39 \\
41 \\
42 \\
63\end{array}$ & $\begin{array}{l}283 \\
310 \\
125 \\
301 \\
237\end{array}$ & $\begin{array}{r}-.1 \\
.1 \\
.0 \\
-.0 \\
-.0 \\
.0\end{array}$ \\
\hline
\end{tabular}

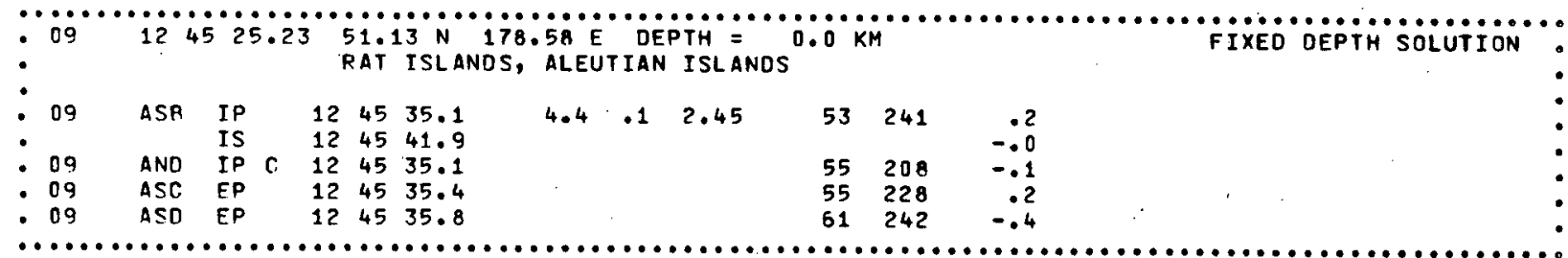

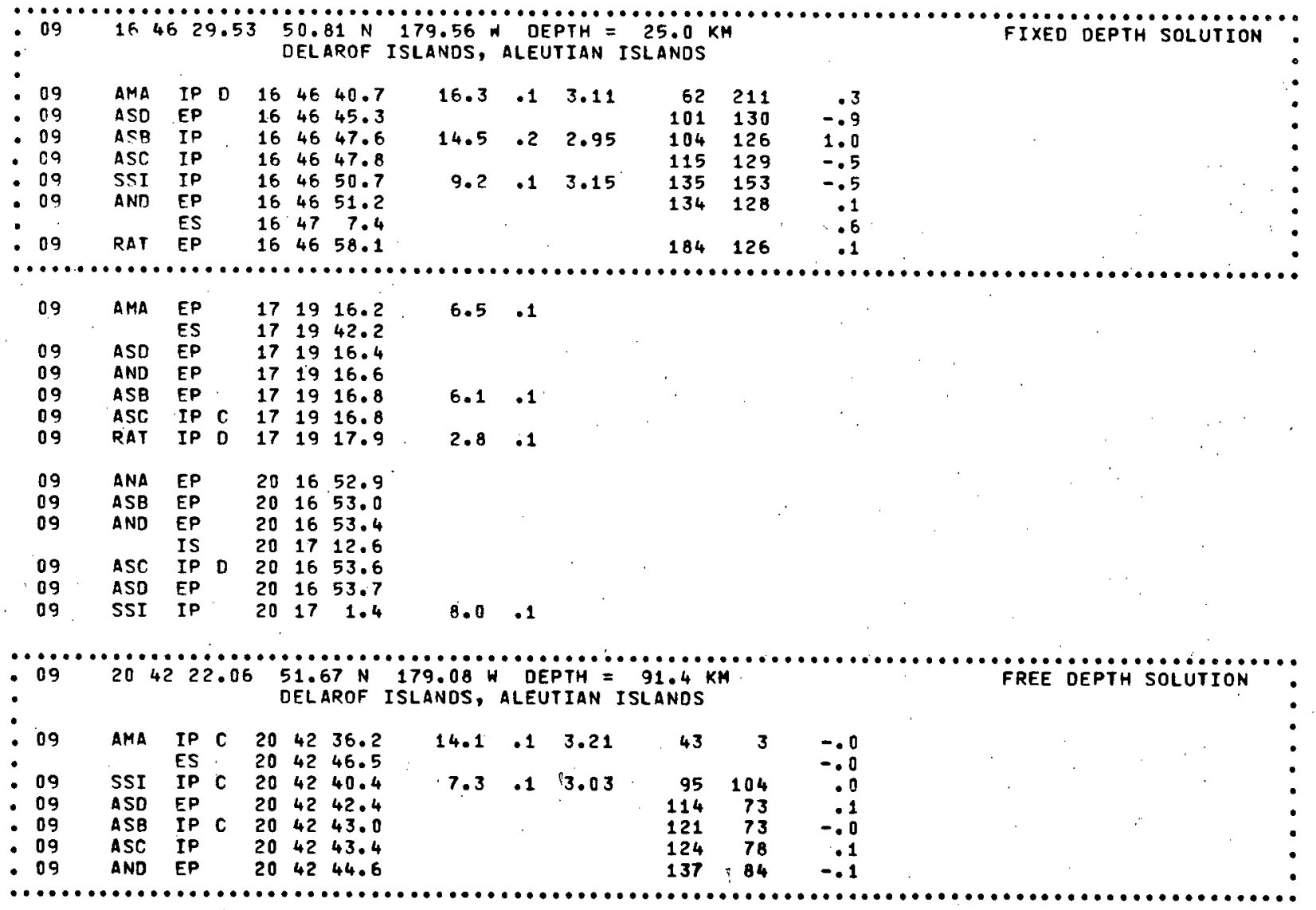




NOV STA PHASE TIME
1970

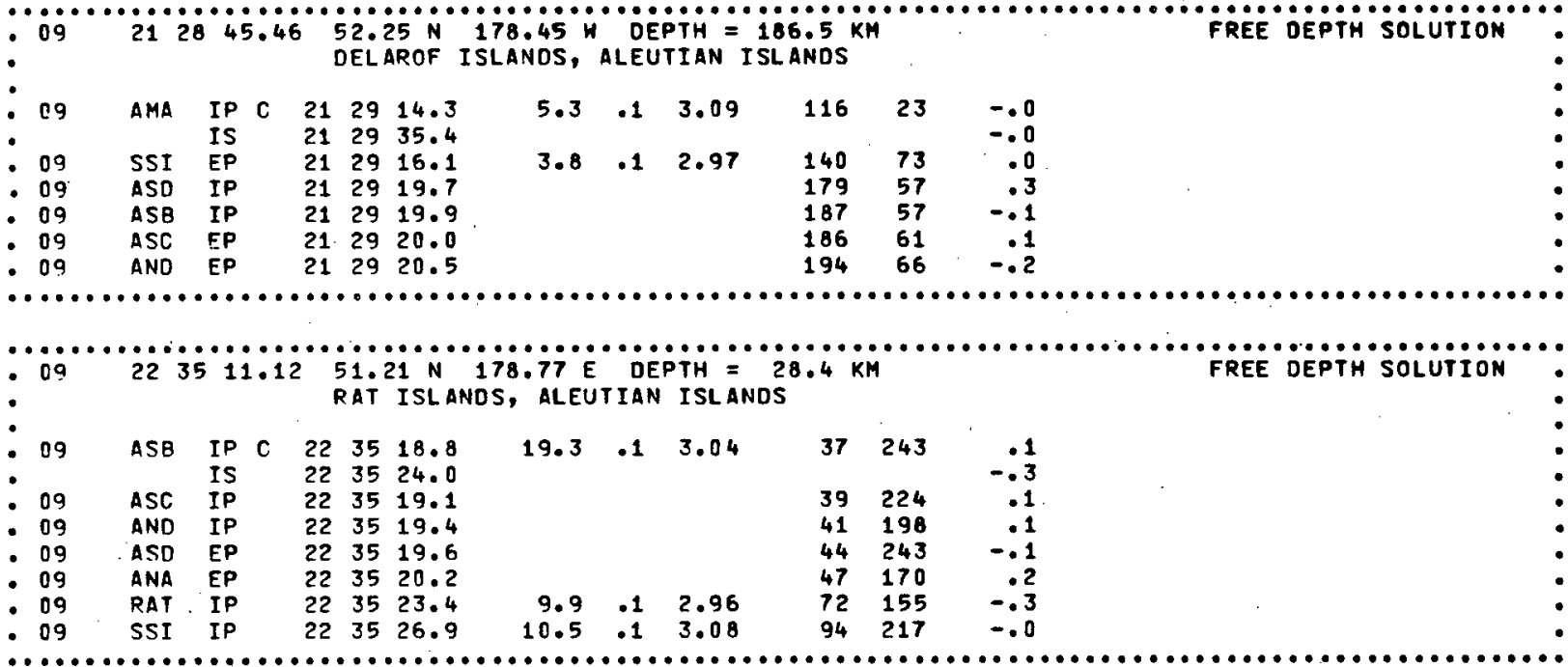

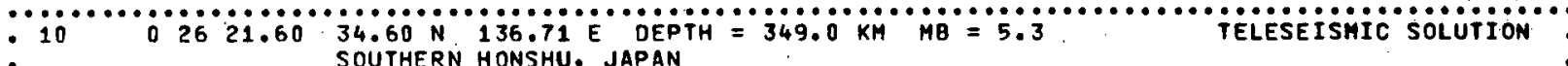
$10 \quad A D K \quad E(P) \quad 00 \quad 33 \quad 2.7$ 4161261

.......................................................................

$\because 10$
$: 10$

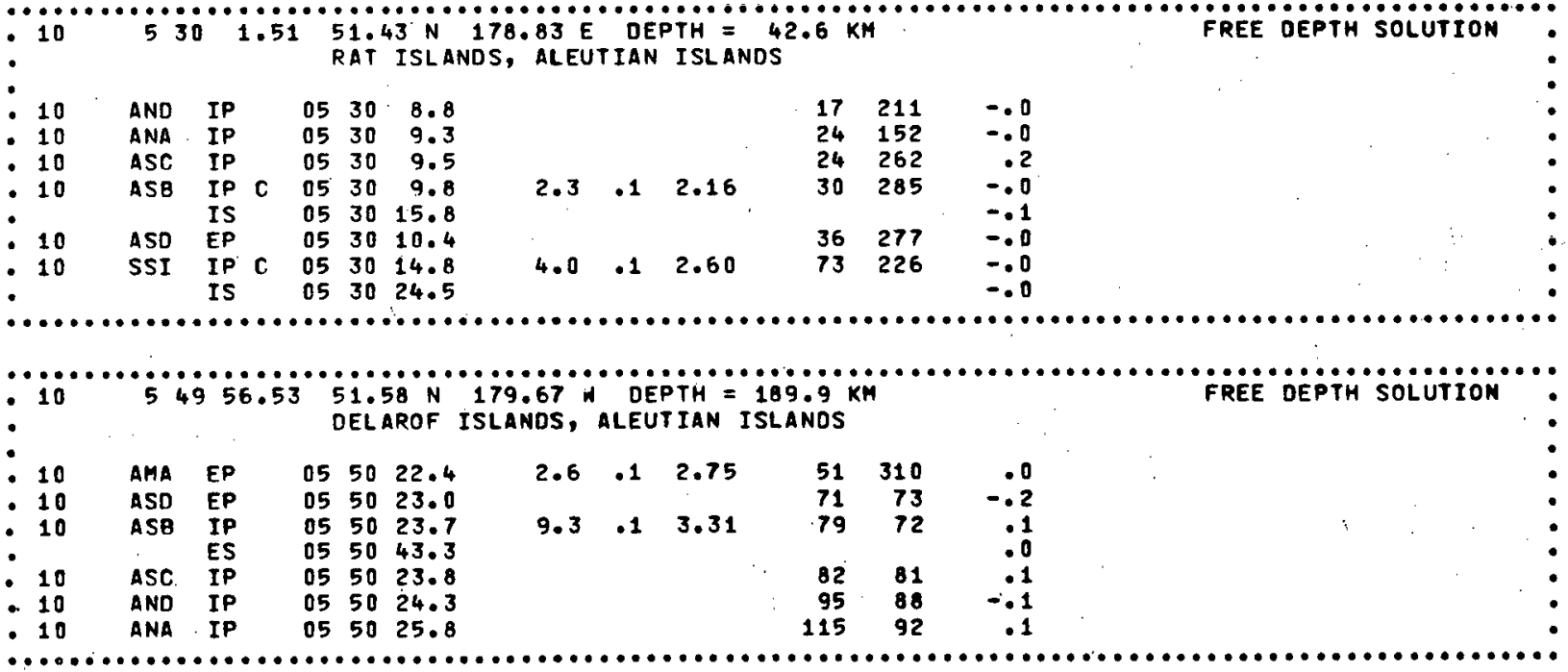




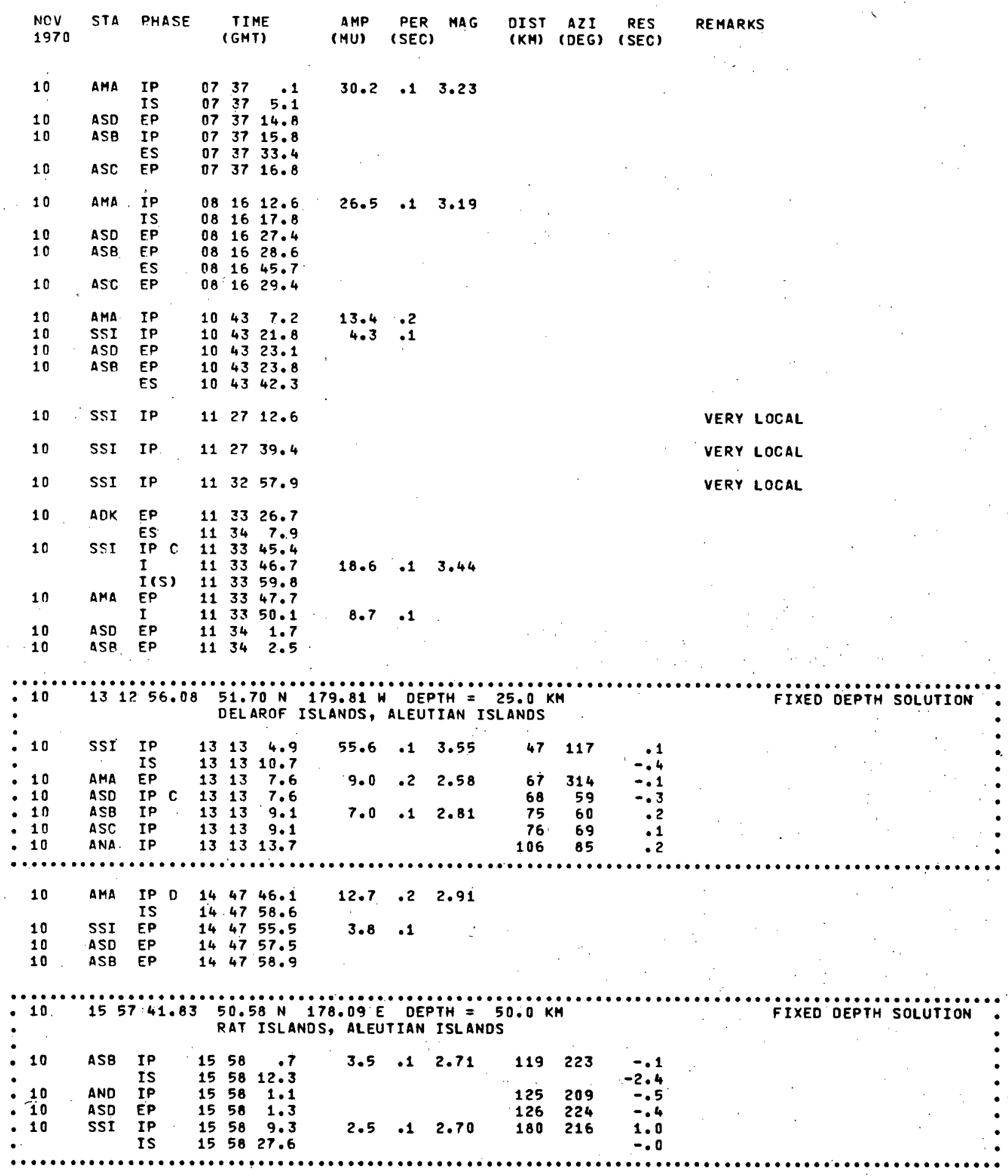




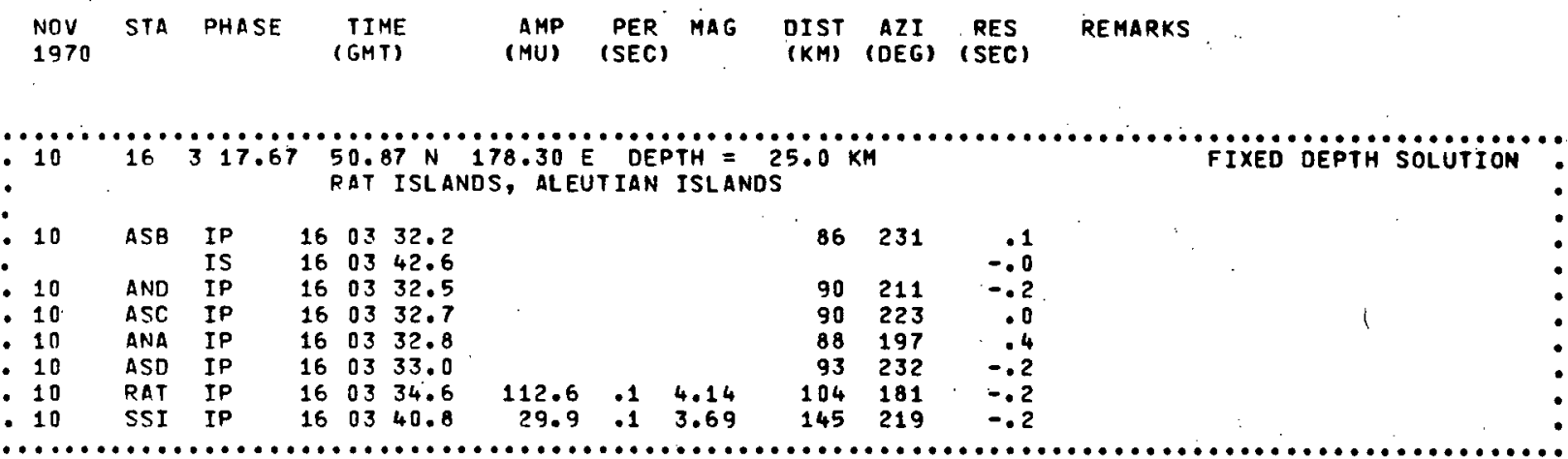

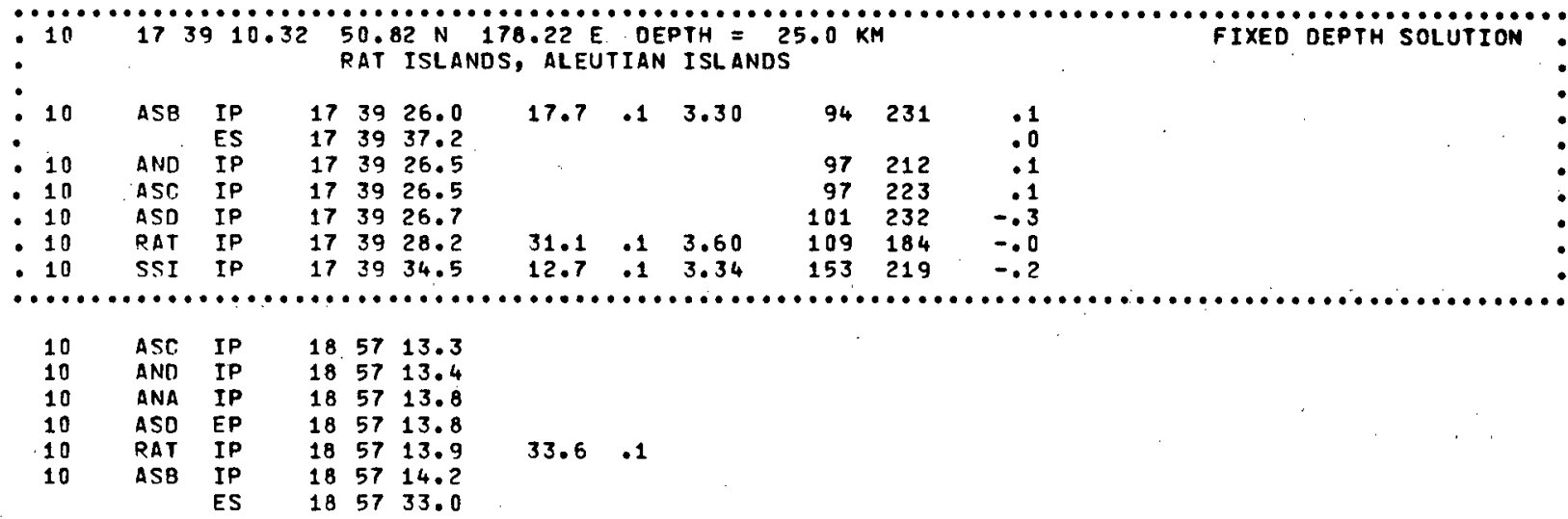

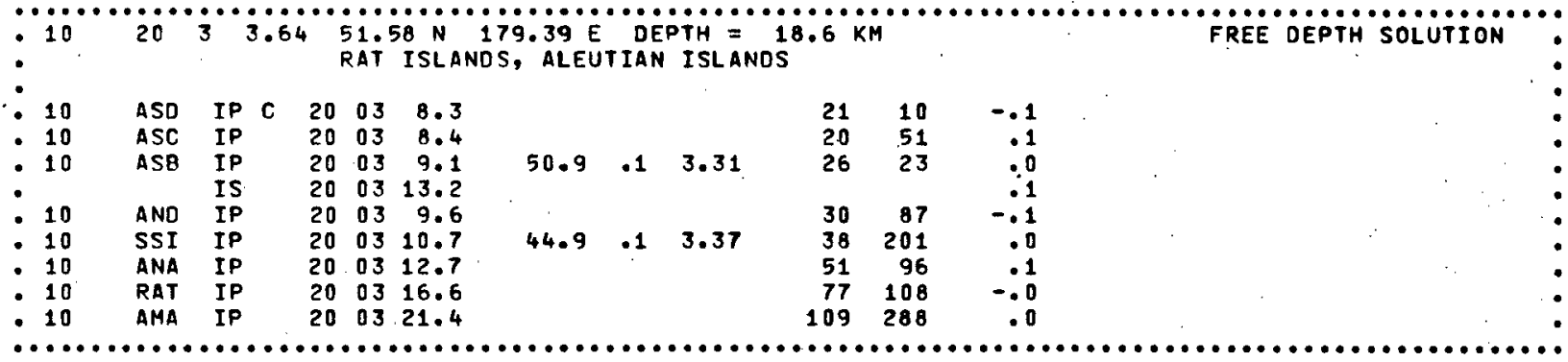

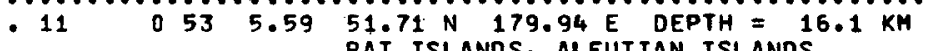
FREE DEPTH SOLUTION

\begin{tabular}{|c|c|c|c|c|c|c|c|c|c|c|c|}
\hline 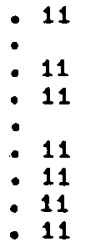 & $\begin{array}{l}\text { SSI } \\
\text { ASO } \\
\text { ASB } \\
\text { AND } \\
\text { AMA } \\
\text { ANA } \\
\text { RAT }\end{array}$ & $\begin{array}{l}\text { IP } \\
\text { IS } \\
\text { IP } \\
\text { IP } \\
\text { IS } \\
\text { IP } \\
\text { IP } \\
\text { IP } \\
\text { IP }\end{array}$ & $\begin{array}{l}\text { D } \\
\text { C }\end{array}$ & $\begin{array}{ll}0 & 0 \\
0 & 0 \\
0 & 0 \\
0 & 0 \\
0 & 0 \\
0 & 0 \\
0 & 0 \\
0 & 0 \\
0 & 0\end{array}$ & $\begin{array}{l}53 \\
53 \\
53 \\
53 \\
53 \\
53 \\
53 \\
53 \\
53\end{array}$ & $\begin{array}{l}11.8 \\
16.2 \\
15.1 \\
16.2 \\
24.0 \\
17.3 \\
19.0 \\
20.4 \\
23.8\end{array}$ & 23.2 & .1 & 3.25 & $\begin{array}{r}32 \\
55 \\
62 \\
70 \\
80 \\
89 \\
112\end{array}$ & $\begin{array}{r}50 \\
51 \\
76 \\
306 \\
84 \\
95\end{array}$ \\
\hline
\end{tabular}




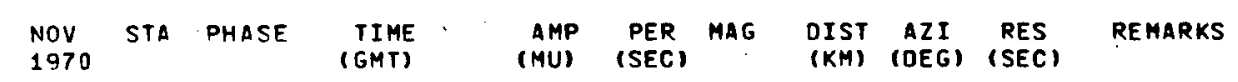

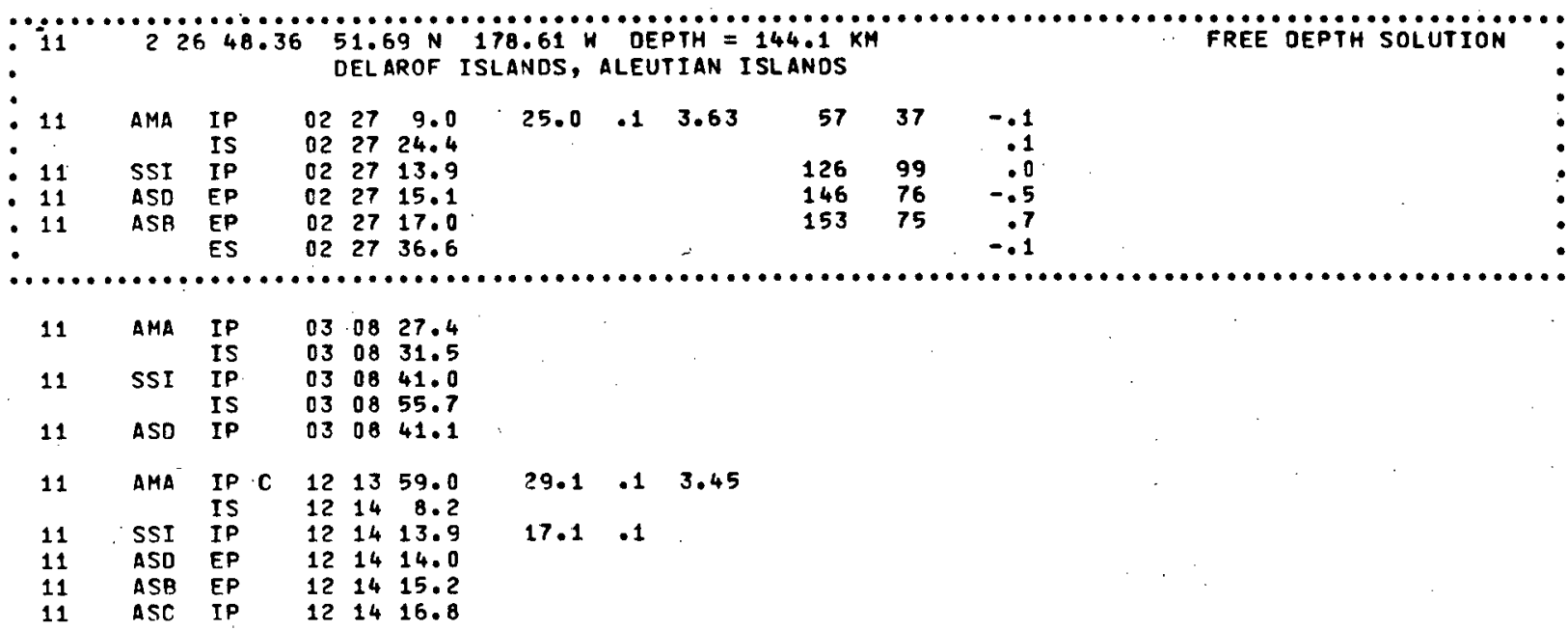

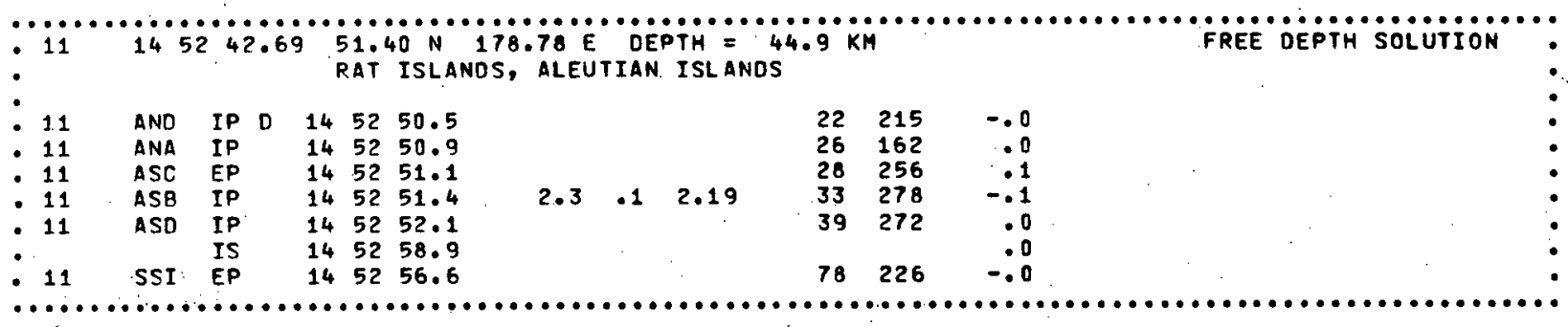

$119773739.8552 .13 \mathrm{~N} 179.24 \mathrm{DEPTH}=50.0 \mathrm{KM}$

FIXED DEPTH SOLUTION

\begin{tabular}{|c|c|c|c|c|c|c|c|c|c|}
\hline \multirow[t]{4}{*}{ SSI } & & $\begin{array}{l}17 \\
17\end{array}$ & $\begin{array}{l}37 \\
38\end{array}$ & $\begin{array}{r}54.7 \\
4.6\end{array}$ & \multirow[t]{4}{*}{3.2} & \multirow{4}{*}{$\begin{array}{l}.1 \\
.1\end{array}$} & \multirow{4}{*}{$\begin{array}{c}2.56 \\
2.88\end{array}$} & 84 & 7 \\
\hline & IP & 17 & 37 & 59.7 & & & & 128 & 4 \\
\hline & $\begin{array}{l}\text { EP } \\
\text { IP }\end{array}$ & $\begin{array}{l}17 \\
17\end{array}$ & $\begin{array}{l}38 \\
38\end{array}$ & $\begin{array}{r}.7 \\
1.7\end{array}$ & & & & $\begin{array}{l}135 \\
139\end{array}$ & $\begin{array}{l}5 \\
6\end{array}$ \\
\hline & Is & 17 & 38 & 17.7 & & & & & \\
\hline
\end{tabular}

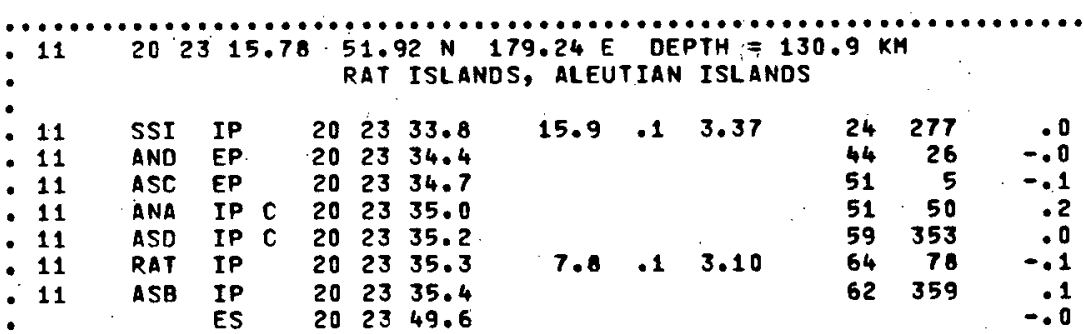




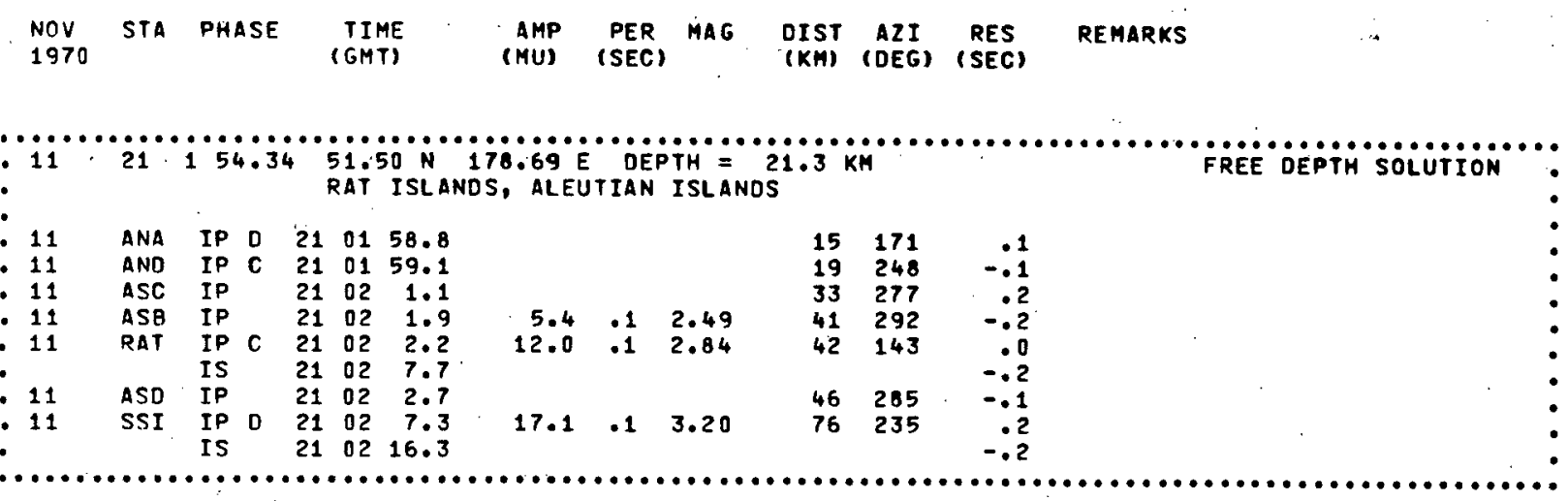

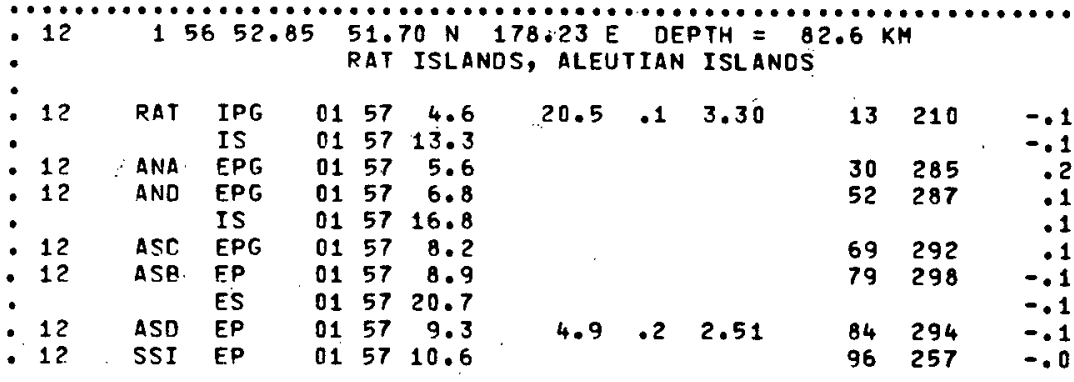

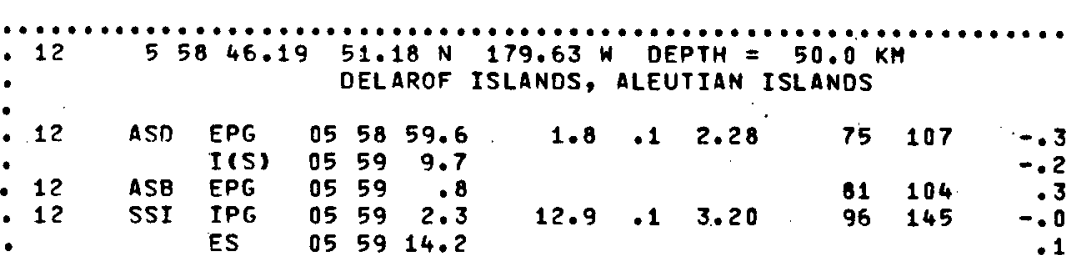

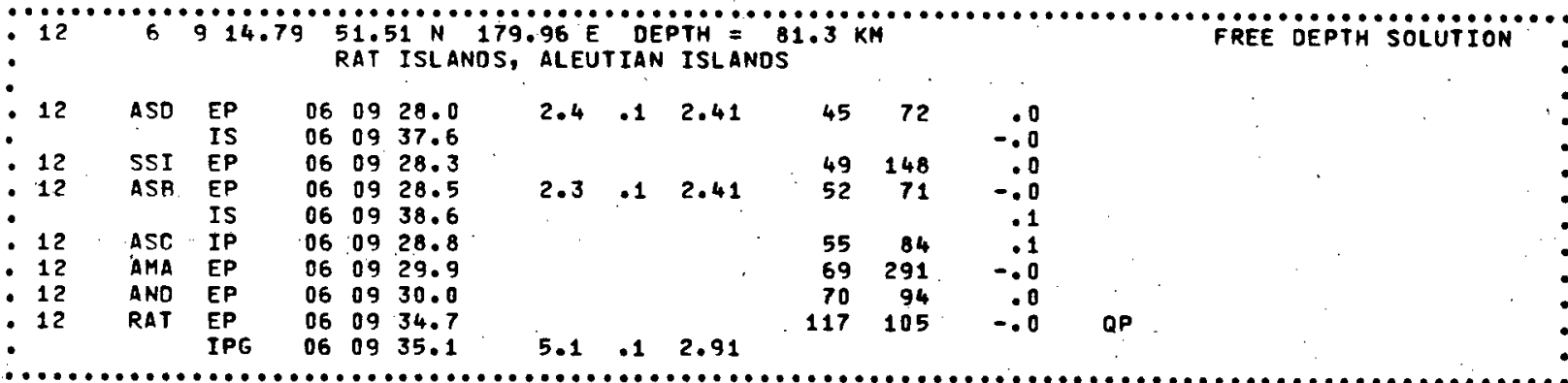

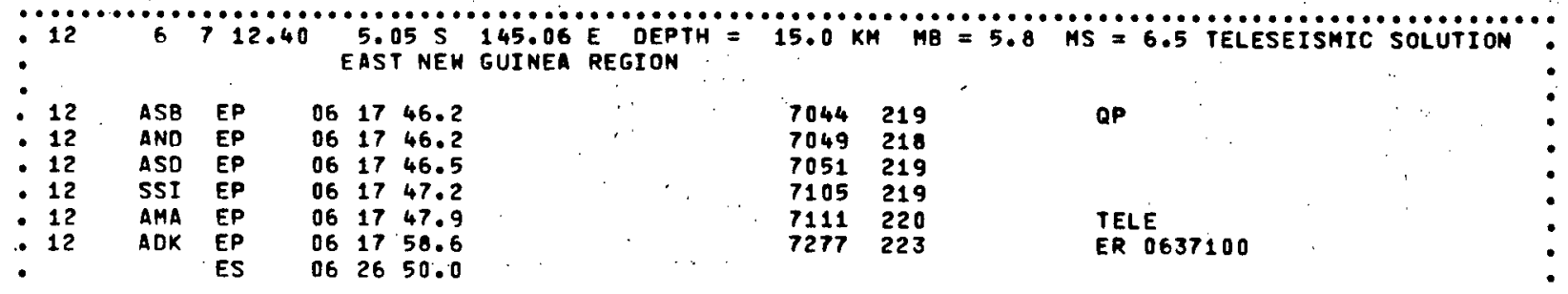




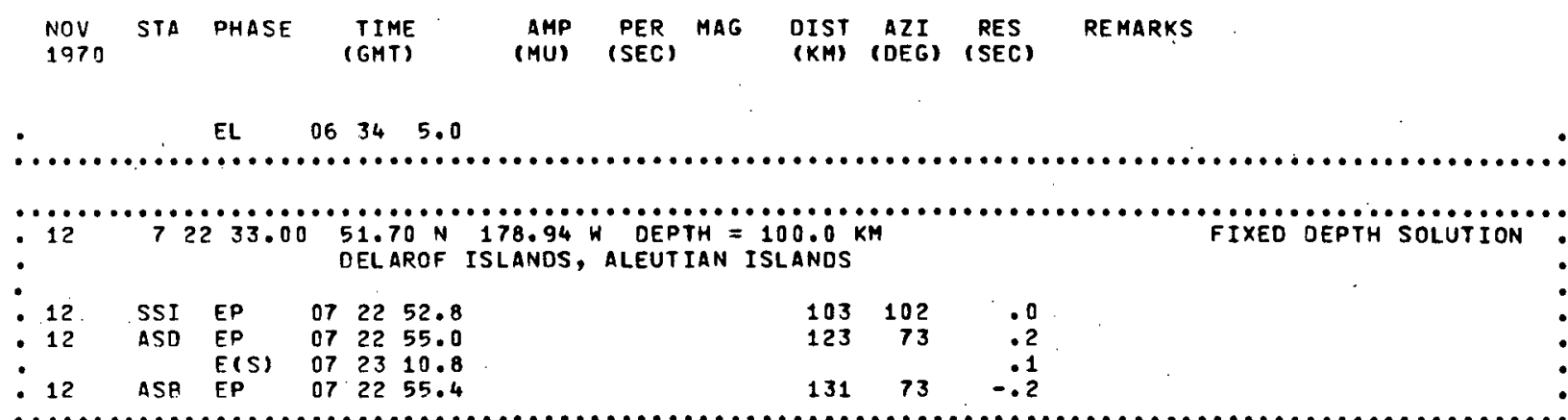

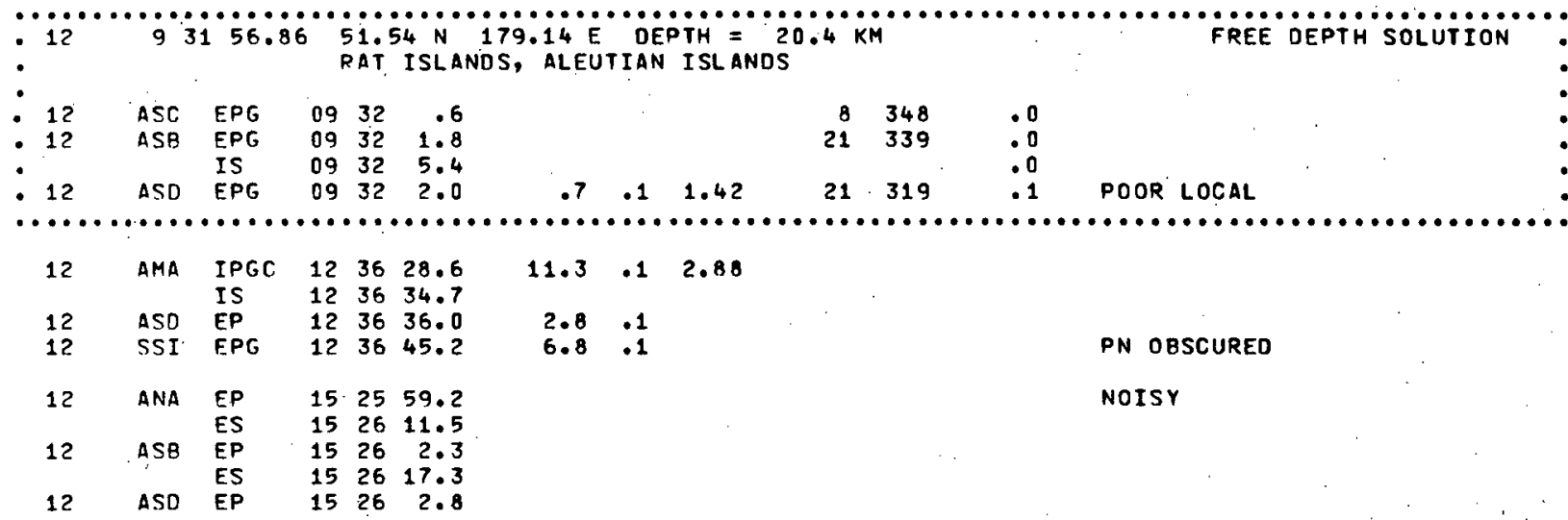

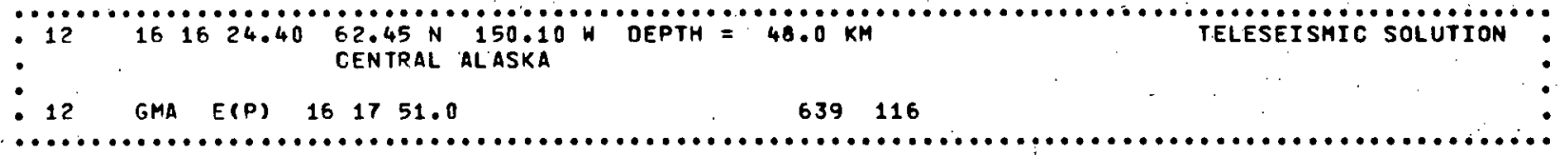

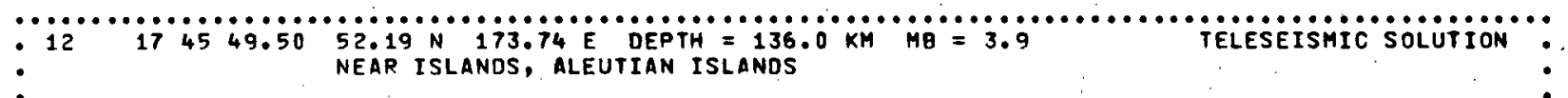

$\begin{array}{lllllll}13 & \text { SSI } & \text { IP } & C & 02 & 22 & 39.3 \\ 13 & \text { AMA } & \text { IP } & 0 & 02 & 22 & 39.5\end{array}$

13 AMA IP D. 02 22 $39.5 \quad$ VERY NOISY

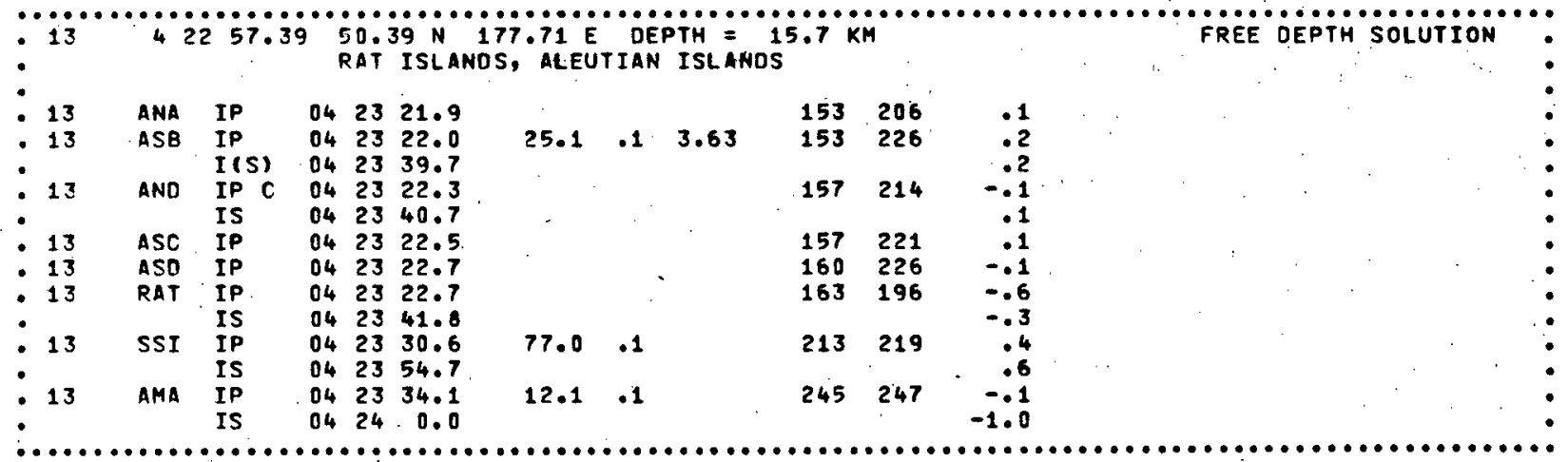

13 SSI EP $10 \quad 0942.7$ 


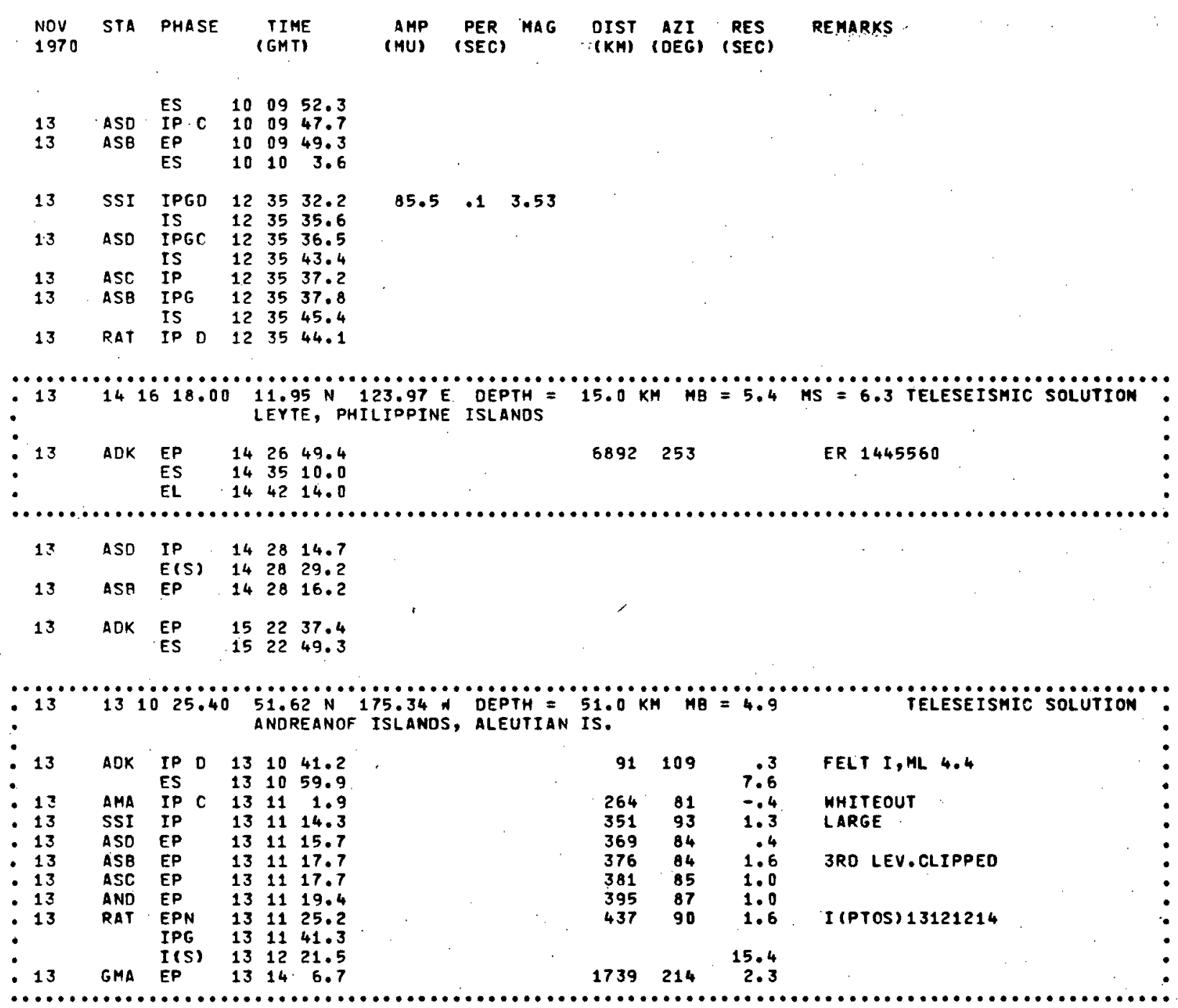

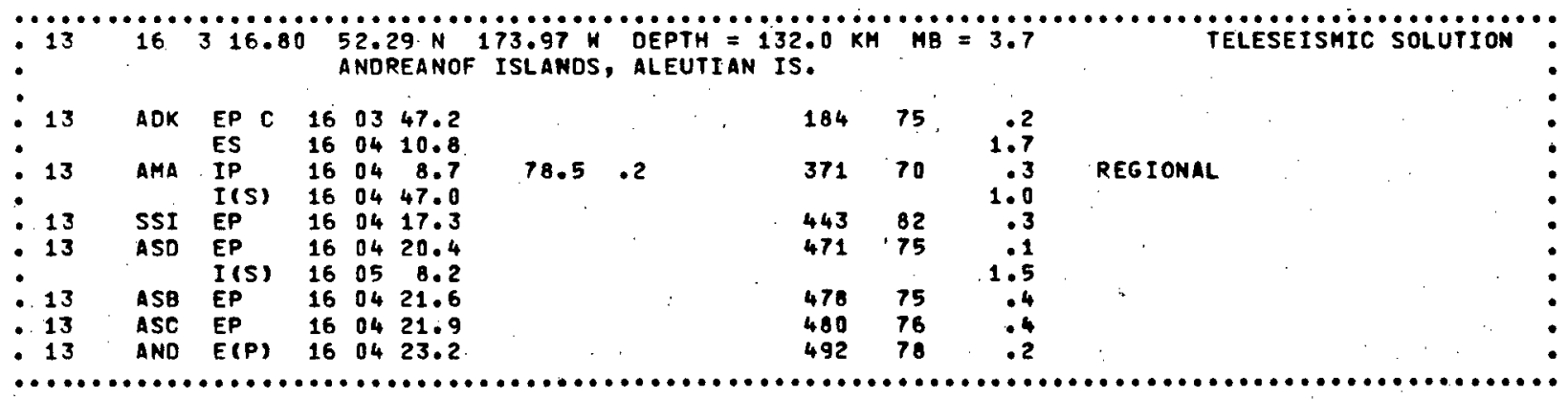




NOV STA PHASE TIME
1970

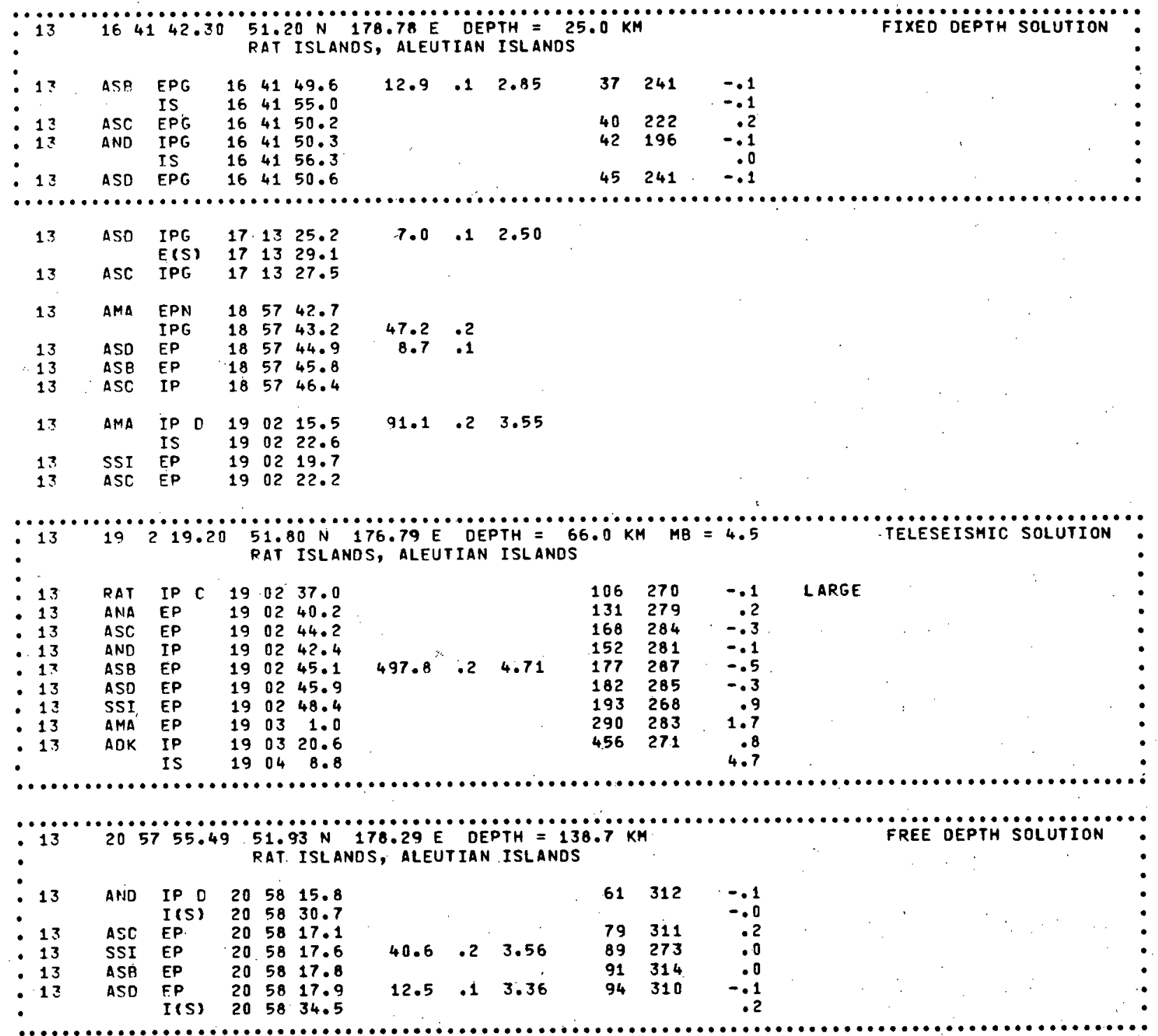

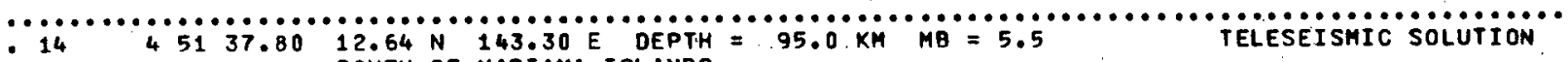
SOUTH OF MARIANA ISLANDS

14 AOK EP $050050.0 \quad 5639234$ 


\begin{tabular}{|c|c|c|c|c|c|c|c|c|c|c|}
\hline $\begin{array}{l}\text { NOV } \\
1979\end{array}$ & STA & PHASE & $\begin{array}{l}\text { TIME } \\
\text { (GMT) }\end{array}$ & $\begin{array}{l}\text { AMP } \\
\text { (MU) }\end{array}$ & $\begin{array}{l}\text { PER } \\
\text { (SEC) }\end{array}$ & HAG & $\begin{array}{l}\text { DIST } \\
\text { (KM) }\end{array}$ & $\begin{array}{c}A Z I \\
\text { (DEG) }\end{array}$ & $\begin{array}{l}\text { RES } \\
\text { (SEC) }\end{array}$ & RE MARKS \\
\hline
\end{tabular}

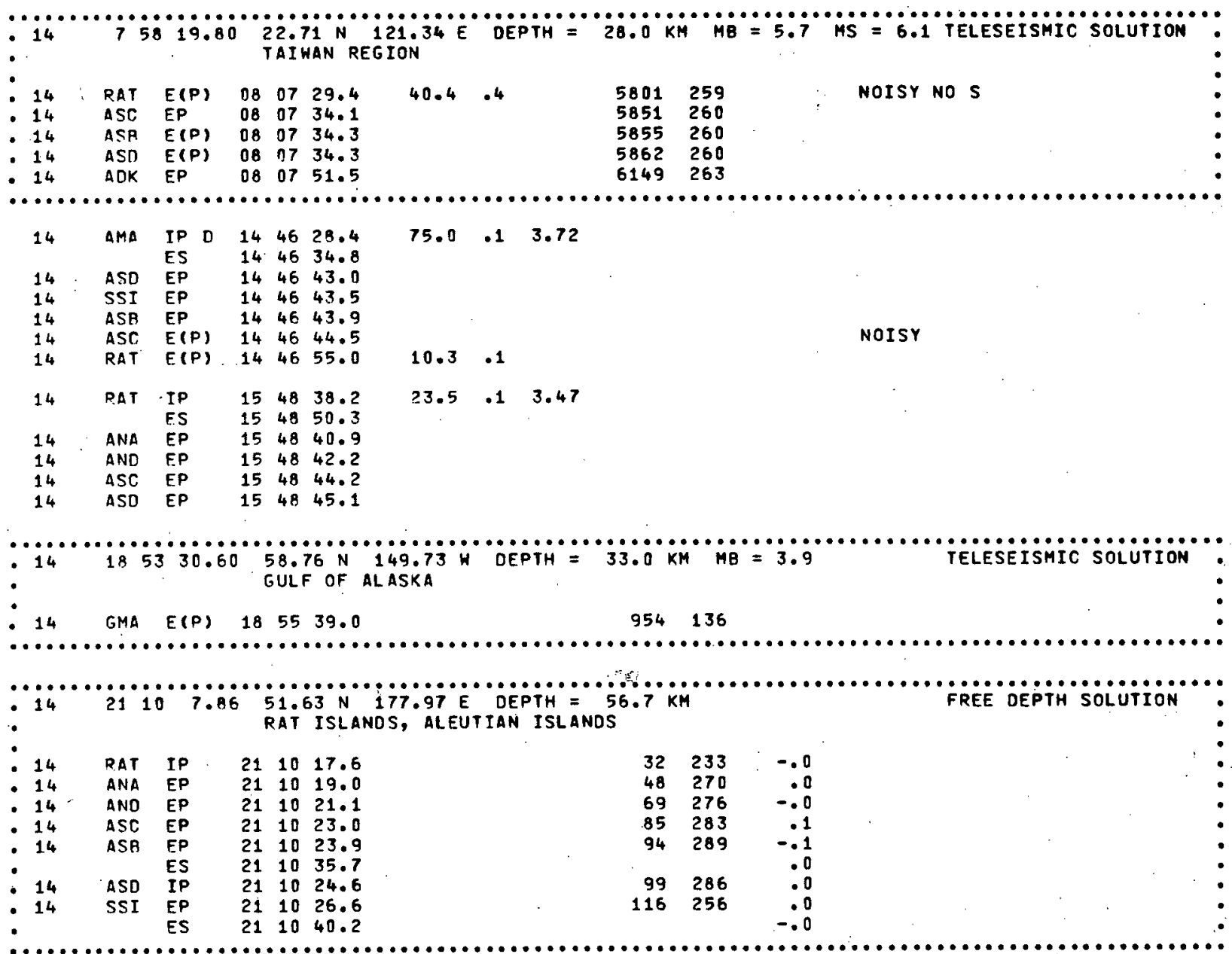

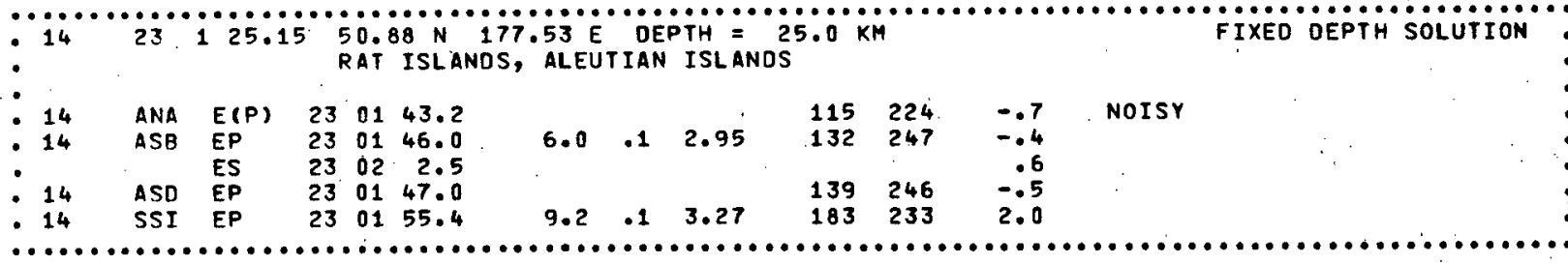

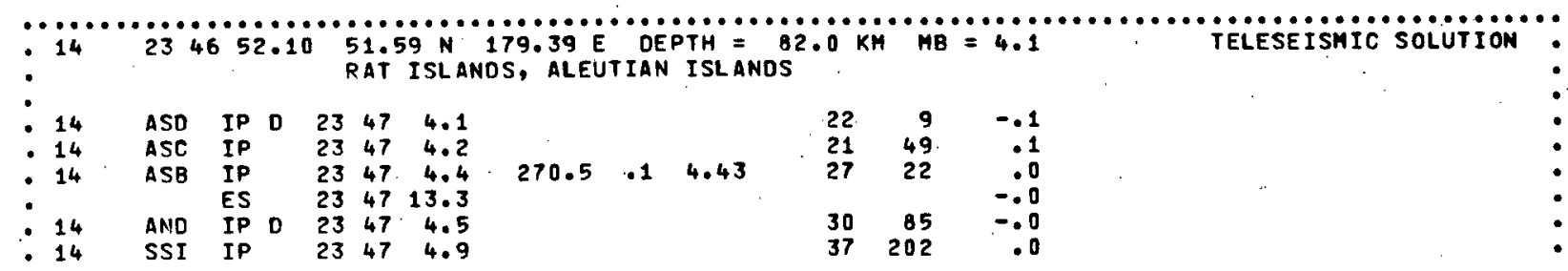




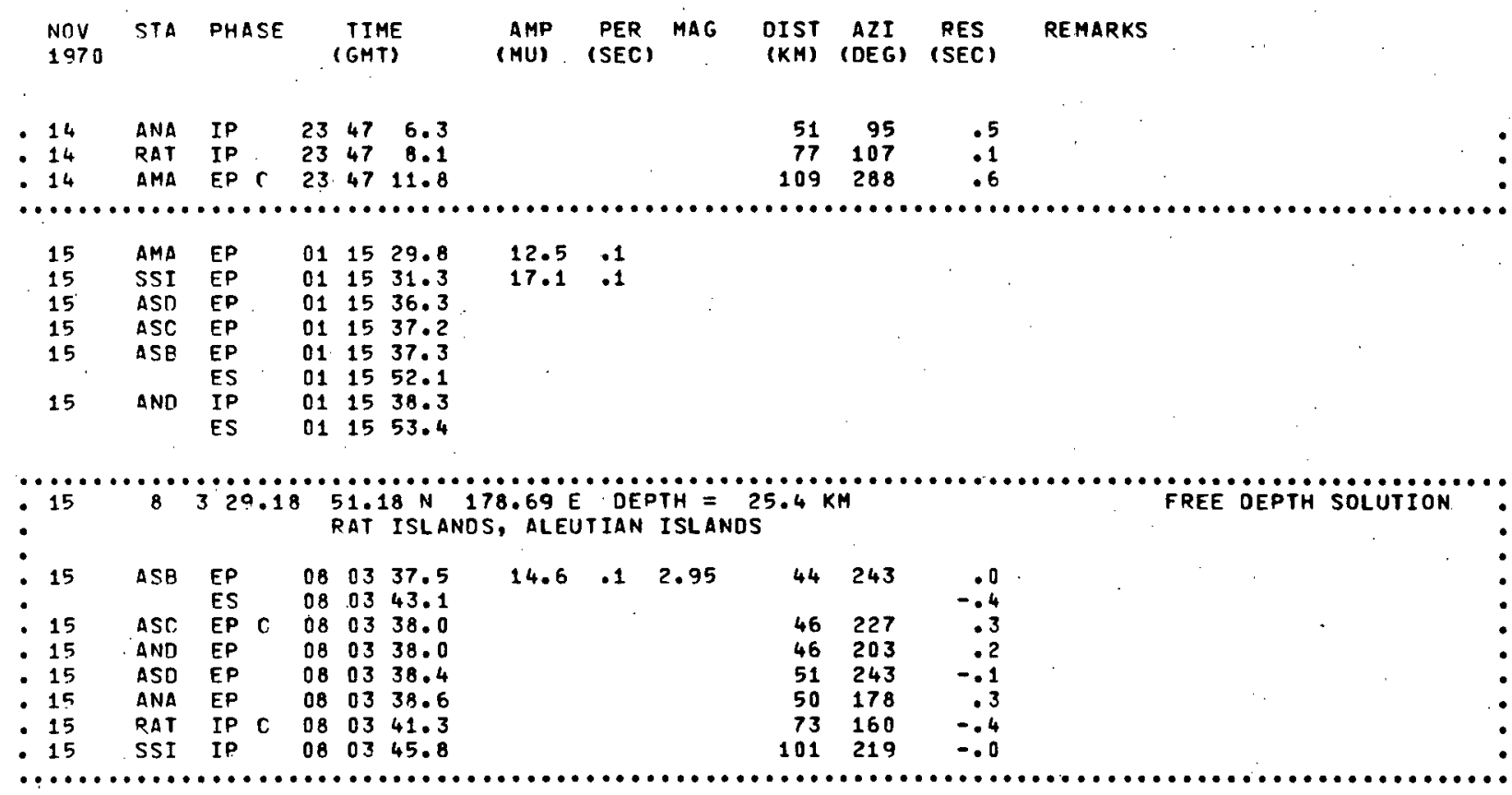

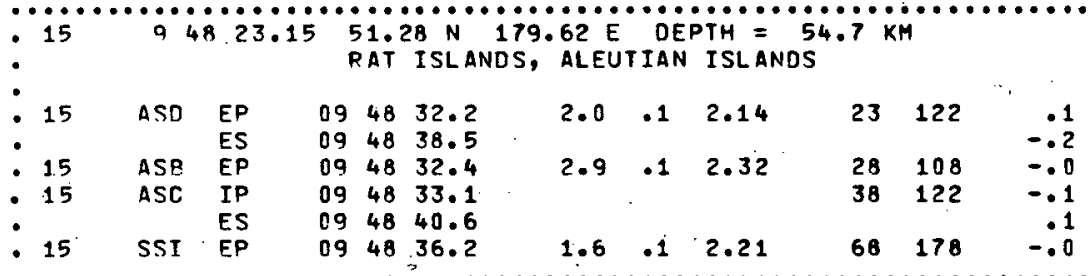

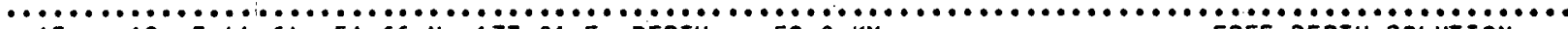
$\begin{array}{lll}15 & 12544.6451 .66 \mathrm{~N} 177.81 \mathrm{E} \text { DEPTH }=59.8 \mathrm{KM} \text { FREE DEPTH SOLUTION }\end{array}$ - RAT ISLANDS, ALEUTIAN islands

\begin{tabular}{|c|c|c|c|c|c|c|c|c|c|c|c|}
\hline $\begin{array}{l}15 \\
15 \\
15 \\
15 \\
15 \\
15 \\
15\end{array}$ & $\begin{array}{l}\text { RAT } \\
\text { ANA } \\
\text { AND } \\
\text { ASC } \\
\text { ASB } \\
\text { ASD } \\
\text { SSI }\end{array}$ & $\begin{array}{l}\text { IP } \\
\text { IP } \\
\text { IP } \\
\text { EP } \\
\text { EP } \\
\text { EP } \\
\text { IP } \\
\text { ES }\end{array}$ & $\begin{array}{l}\mathrm{C} \\
\mathrm{C}\end{array}$ & $\begin{array}{l}12 \\
12 \\
12 \\
12 \\
12 \\
12 \\
12 \\
12\end{array}$ & $\begin{array}{l}05 \\
05 \\
05 \\
06 \\
06 \\
06 \\
06 \\
06\end{array}$ & $\begin{array}{r}55.2 \\
57.1 \\
59.2 \\
1.6 \\
1.9 \\
2.7 \\
4.6 \\
19.1\end{array}$ & $\begin{array}{l}2.9 \\
4.3\end{array}$ & $\begin{array}{r}.1 \\
.1\end{array}$ & $\begin{array}{l}2.60 \\
2.82\end{array}$ & $\begin{array}{r}39 \\
59 \\
80 \\
96 \\
105 \\
110 \\
125\end{array}$ & $\begin{array}{l}246 \\
274 \\
278 \\
283 \\
289 \\
286 \\
259\end{array}$ \\
\hline
\end{tabular}

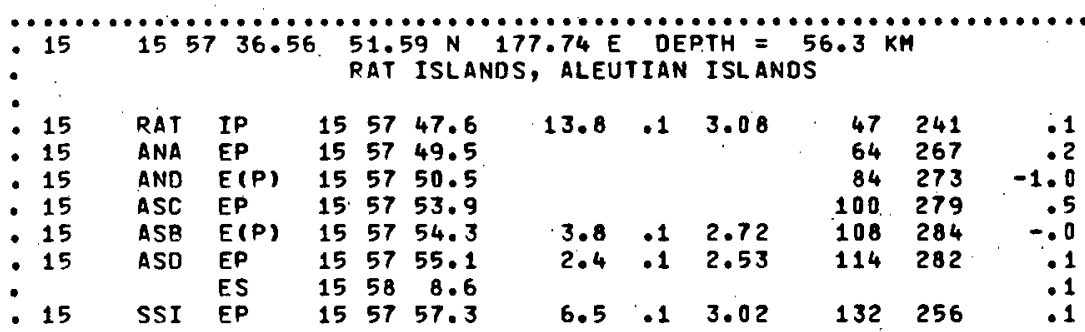




$\begin{aligned} & \text { NOV } \\ & 1970\end{aligned}$
STA PHASE

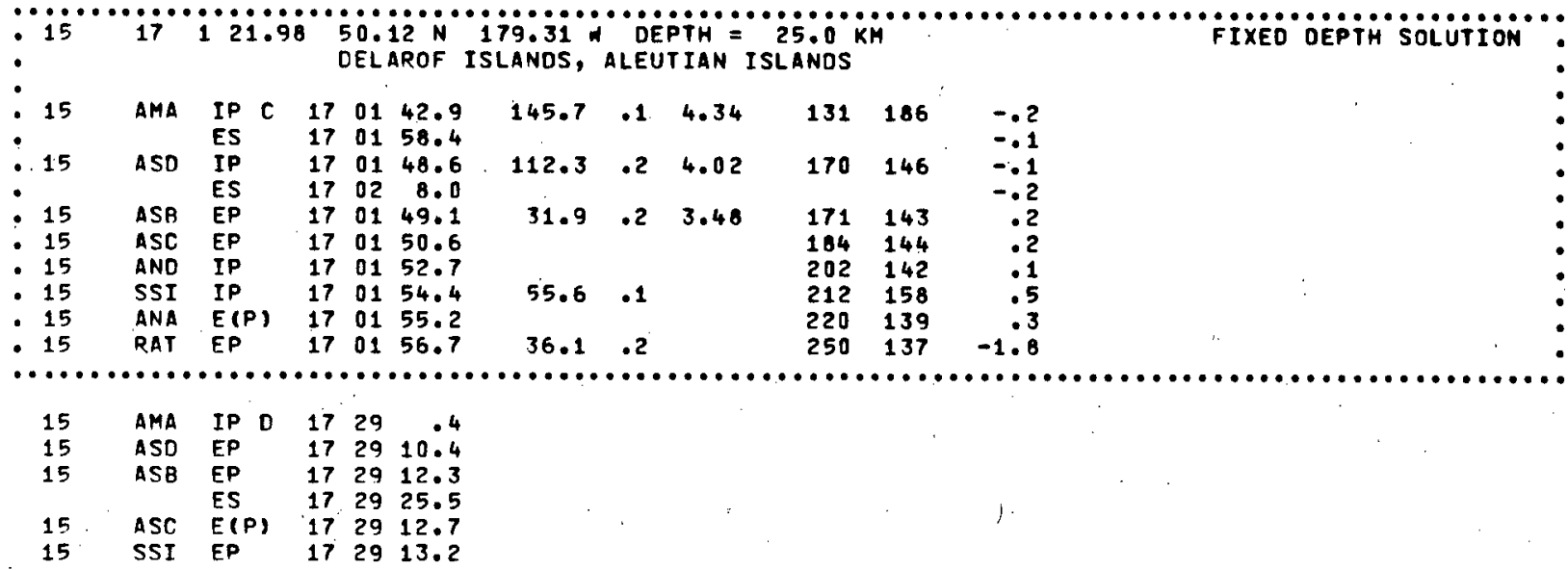



$.15224348 .6351 .10 \mathrm{~N} 179.98 \mathrm{HEPTH}=35.5 \mathrm{kM}$

FREE DEPTH SOLUTION

$\begin{array}{llllllll}15 & \text { ASD IP } & 22 & 43 & 59.3 & 57 & 124 & -01\end{array}$

15 ASB EP 224359.9

- 15 AMA IP D $2244 \quad .7$

- 22448.3

- 15 SSI IP $2244 \quad 4.6$

15 RAT EP 22441007

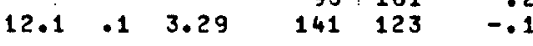

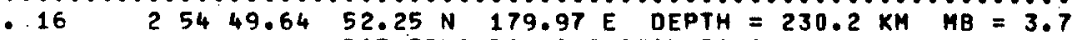

- RAT ISLANDS, aleutian ISLANDS

- 16 SSI IP $0 \quad 025520.3$

- 16 AND IP D 025522.5

- 16 ASO IP C 025522.6

- 16 ASB IP C 025522.9

- I(S) 025547.1

- 16 ANA IP C 025523.1

- 16 Rat IP 02.5523 .4

- 16 AMA IP D 025523.6

$48 \quad 33 \quad 1 \quad$ NHITE OUT

•........

$\begin{array}{lllllllll}16 & \text { AMA } & \text { EP } & 03 & 16 & 48.3 & 66.0 & .2 & : \\ 16 & \text { SSI } & \text { EP } & 03 & 17 & 25.6 & 03 & 16 & 58.3 \\ 16 & \text { ASD } & \text { EP } & 03 & 17 & 1.6 \\ 16 & \text { ASB } & \text { EP } & 03 & 17 & 2.5 \\ 16 & \text { GMA } & \text { E(P) } & 06 & 29 & 14.0\end{array}$

$\because 16$
$\vdots$
$\square$

$\begin{array}{llllll}16 & \text { RAT IP } & 08 & 04 & 15.9 \\ & & I(S) & 08 & 04 & 34.5\end{array}$ 


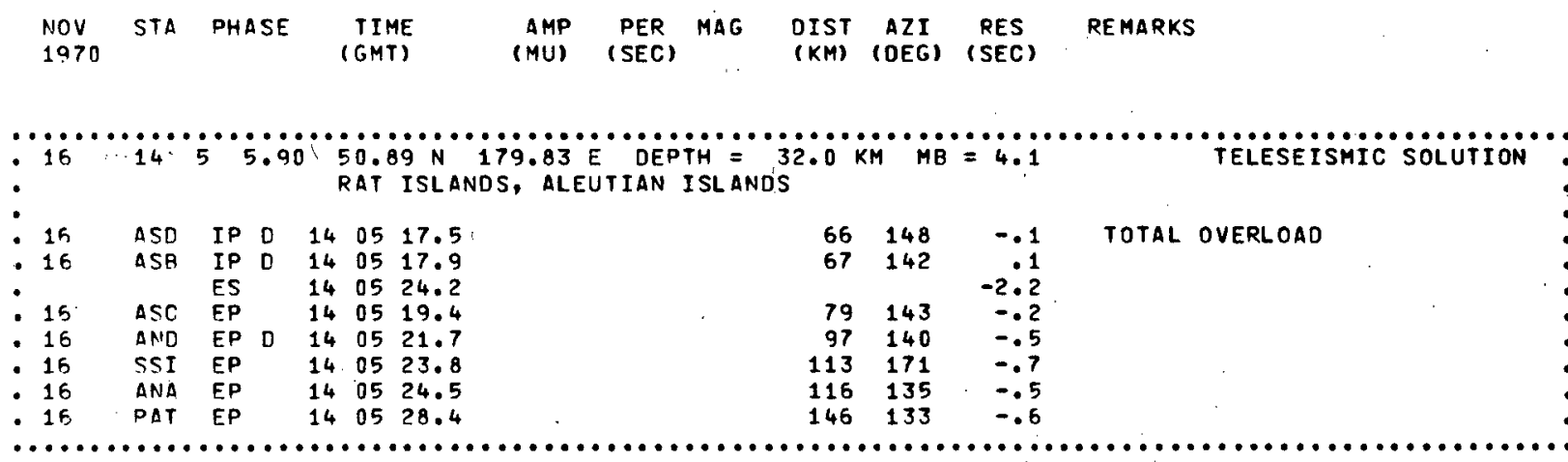

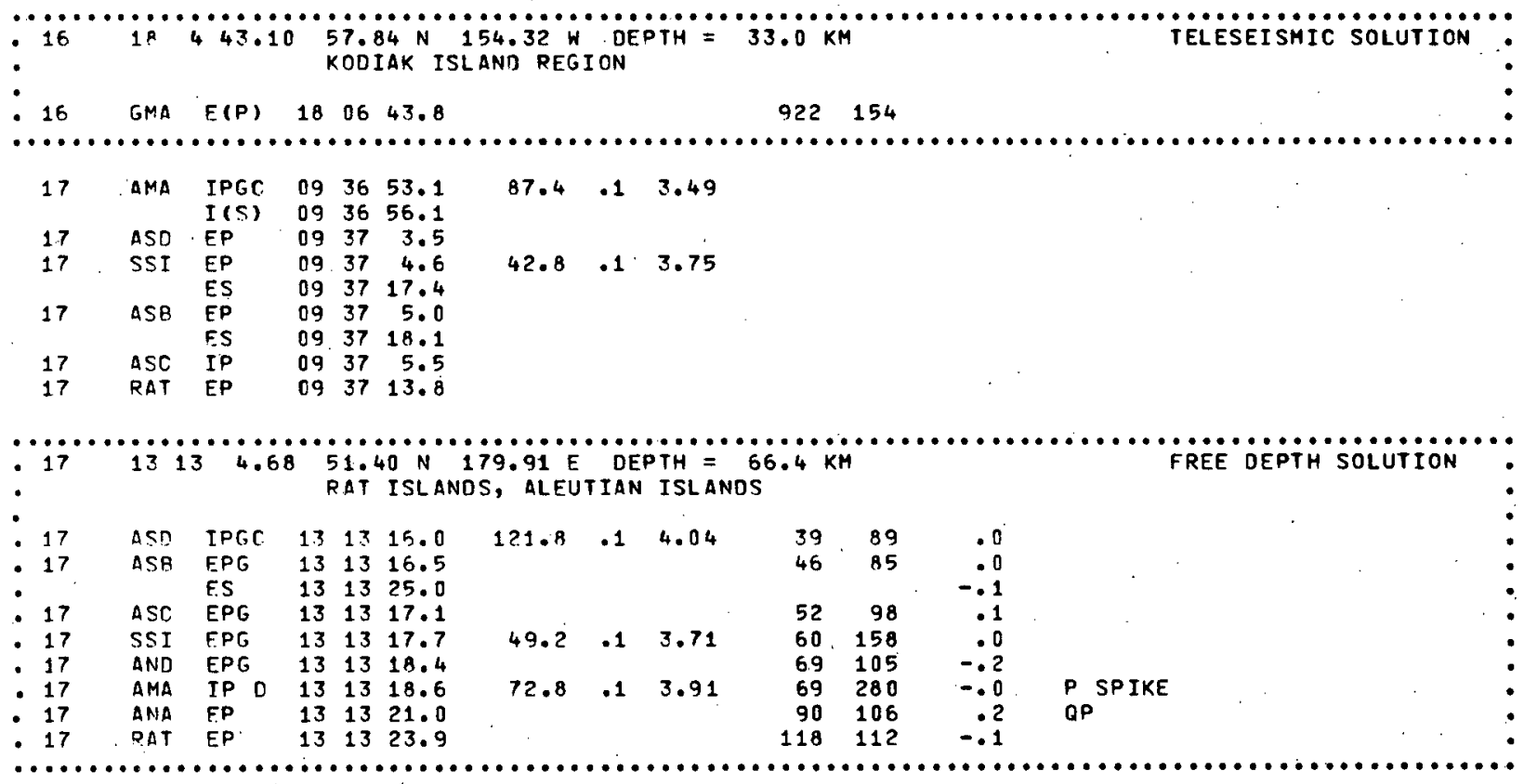

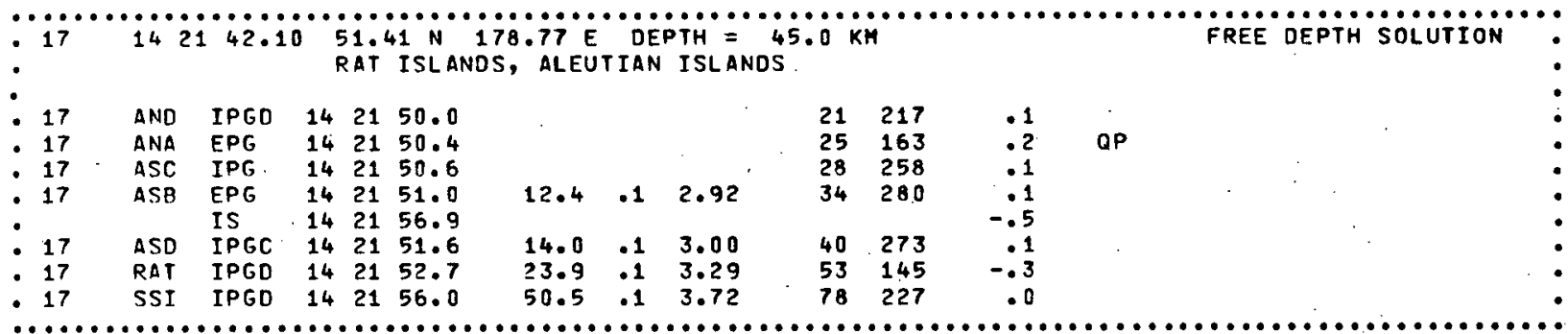

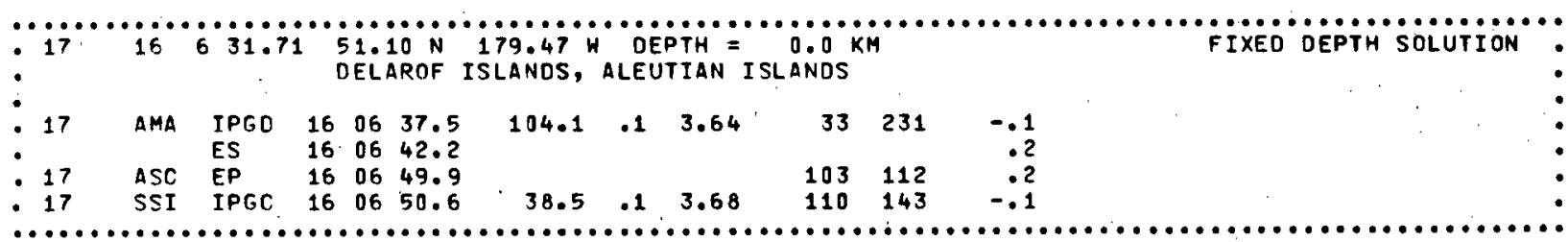




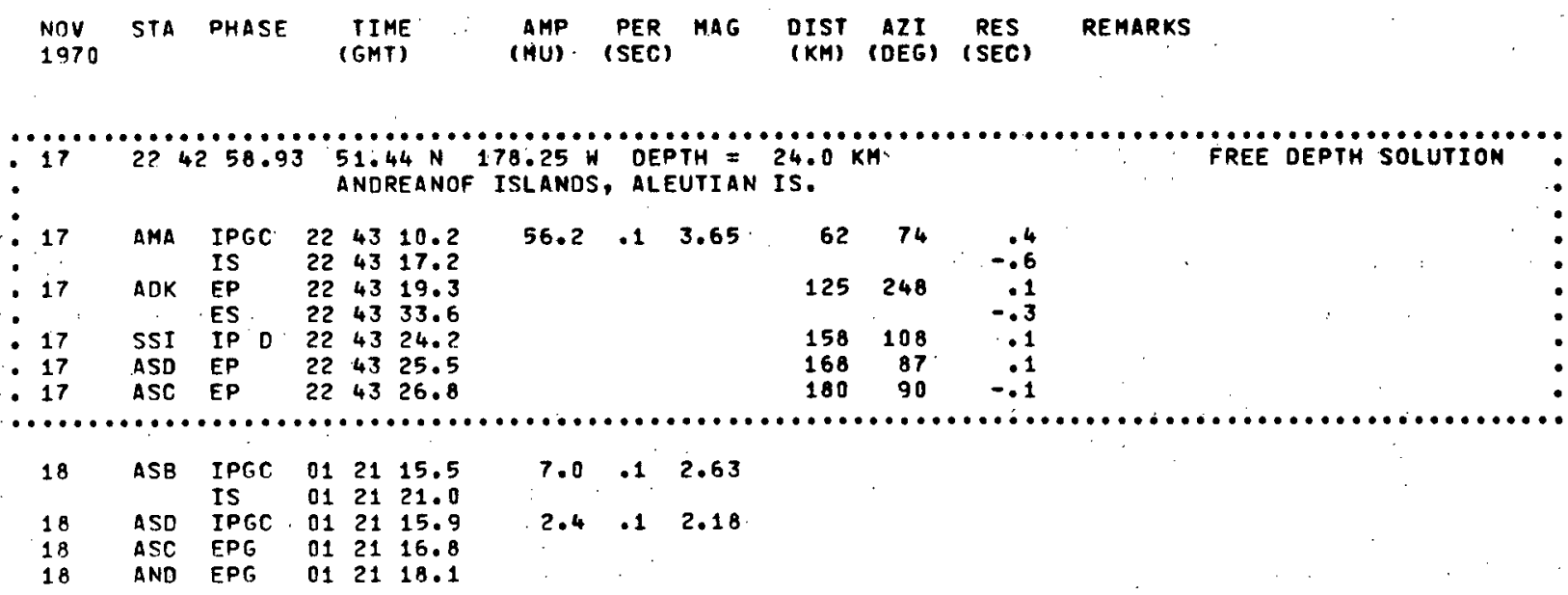

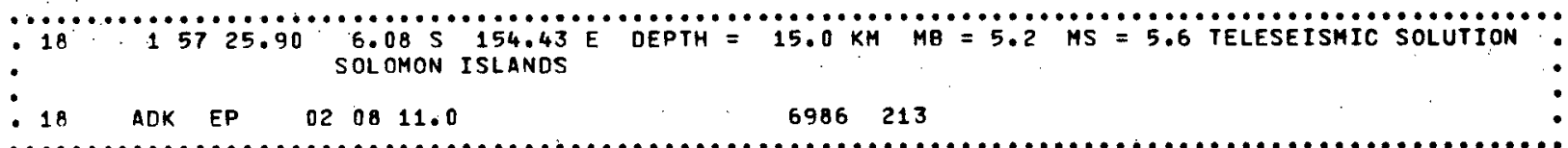

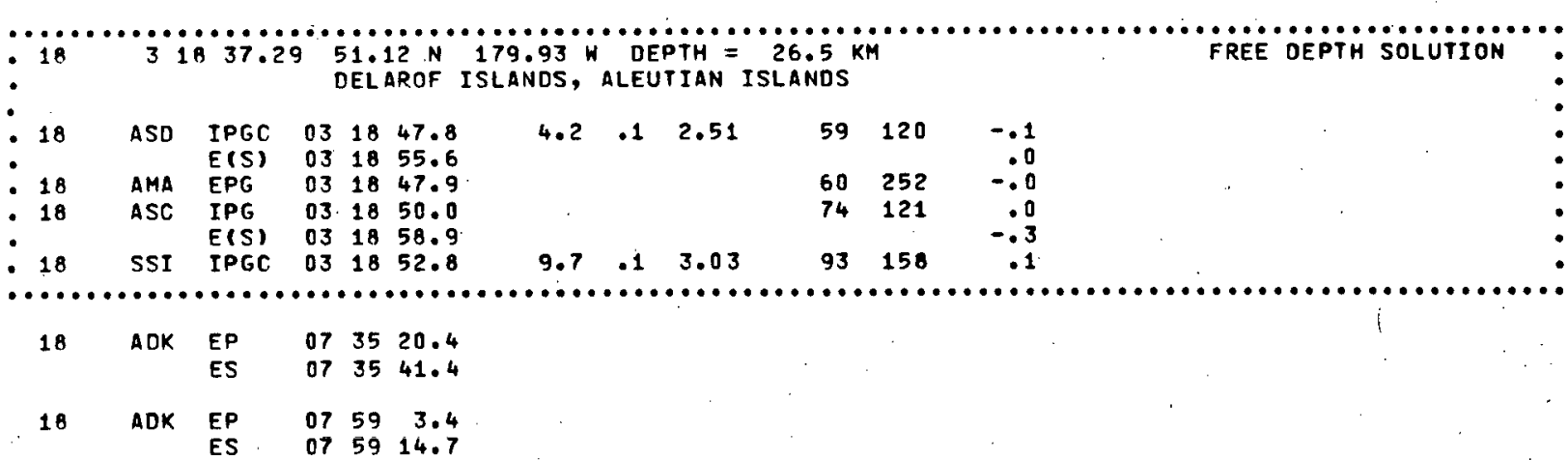

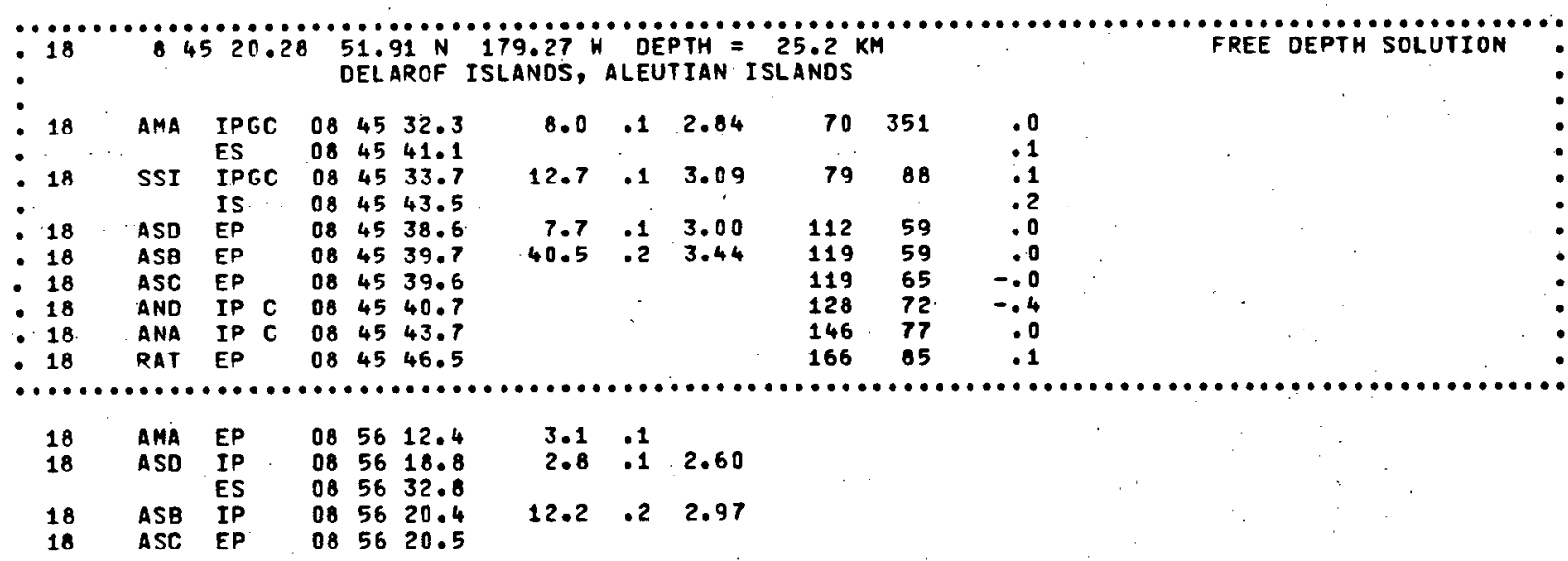




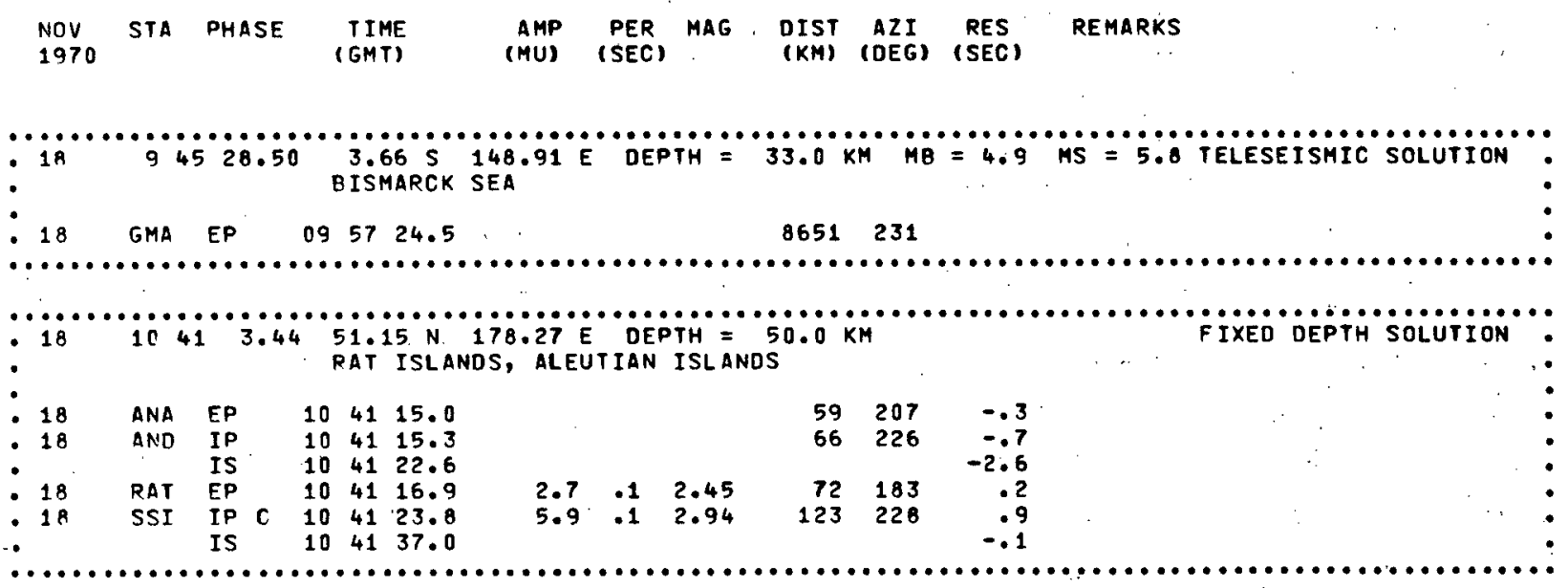

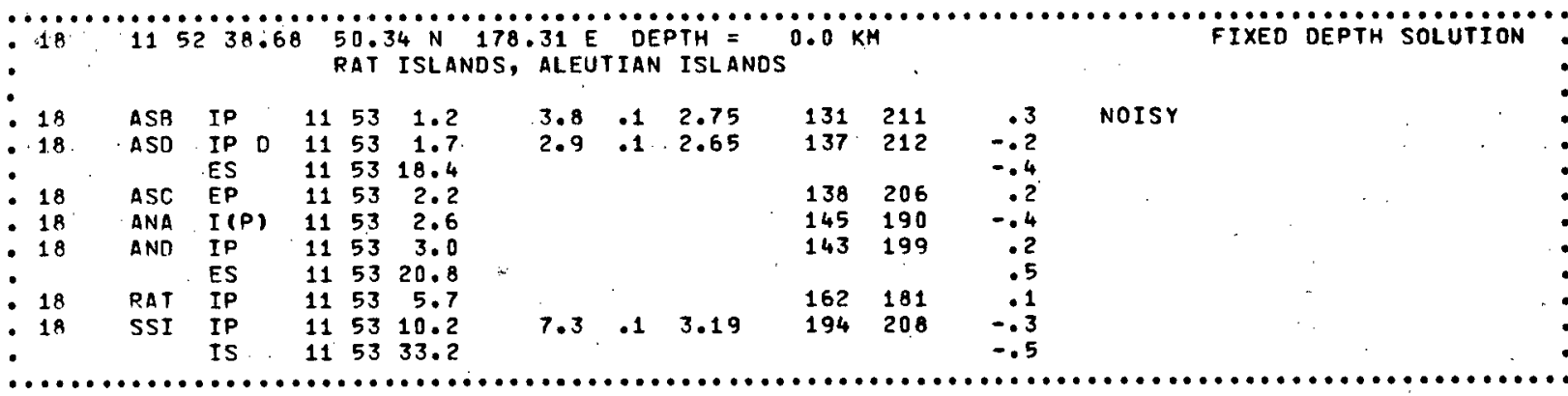

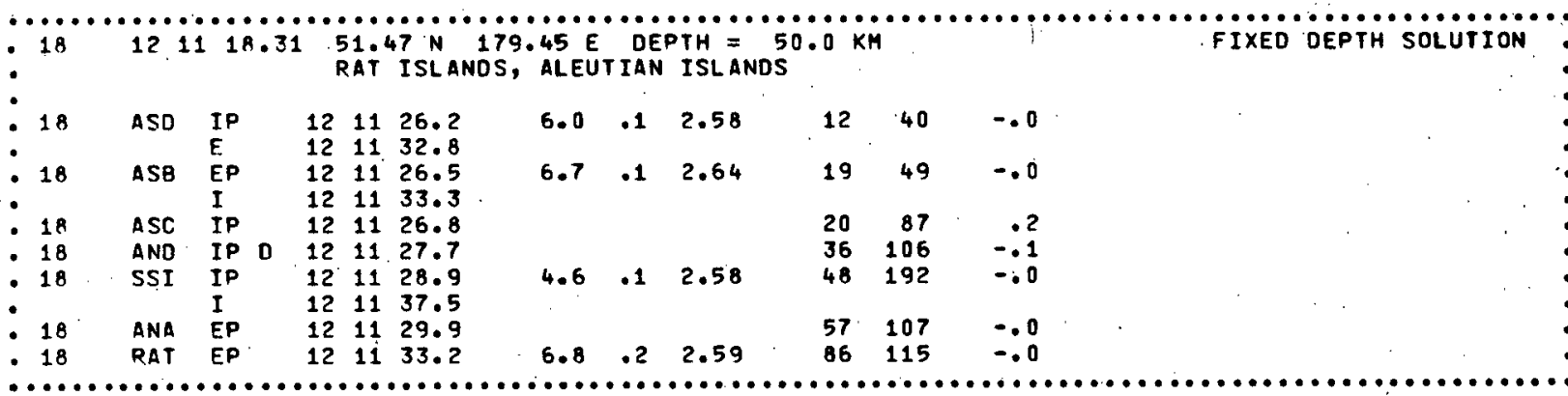

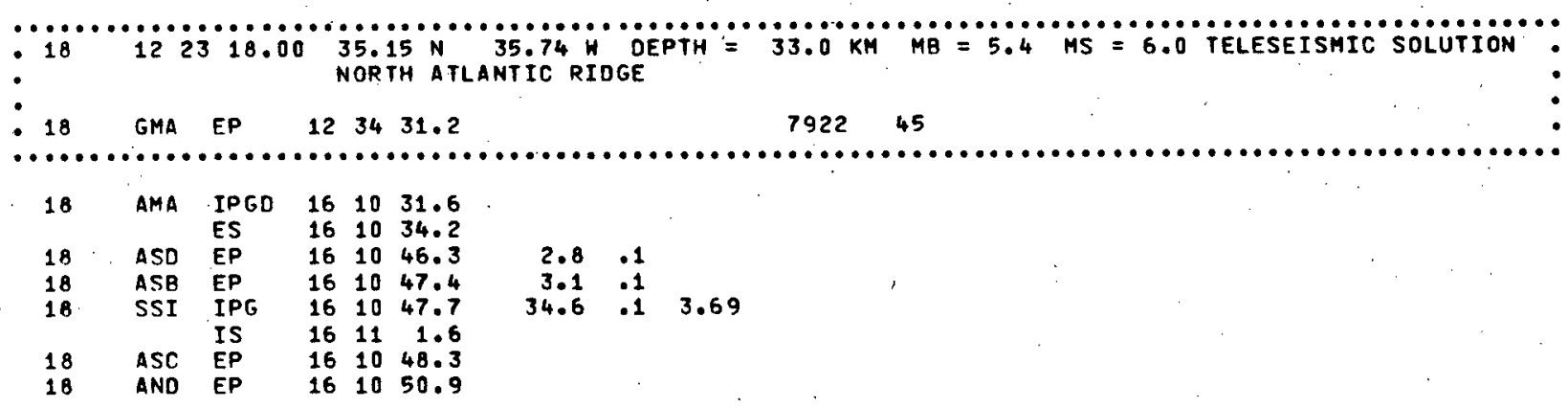




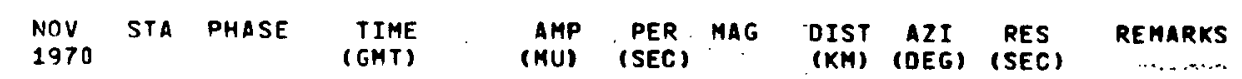

$\because 18$
$\vdots$

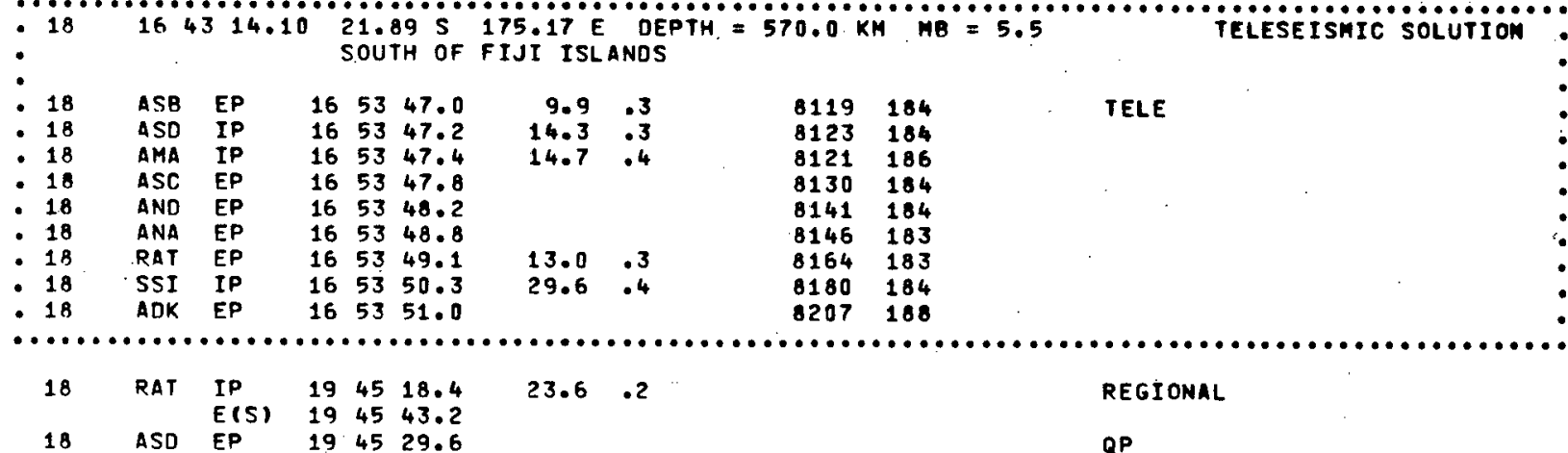

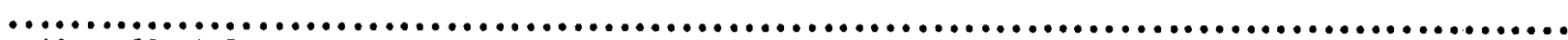

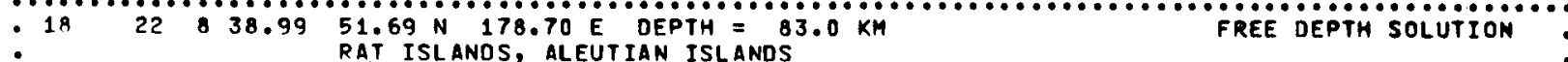

- 18 ana iP 220851.0

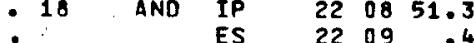

- 18 Rat IP C 220851.3

- is 2209.3

. 18 ASC IP D 22 OB 52.3

- 18 ASB EP 220852.8

- 18 ASD IP D 220853.1

- ES 22093.2

$\begin{array}{lllllllllllllll}18 & \text { SSI EP } & 22 & 0 B & 53.9 & 2.6 & .1 & 2.49 & 65 & 250 & -.2\end{array}$

...............................................

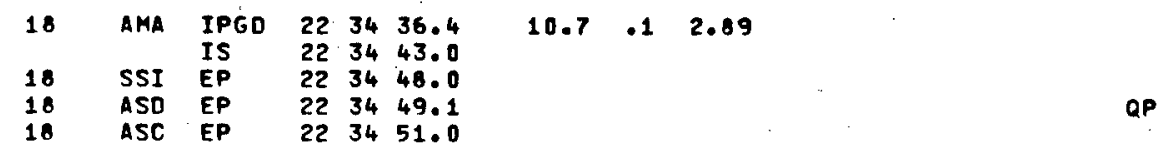

$\because 18$
$\vdots$

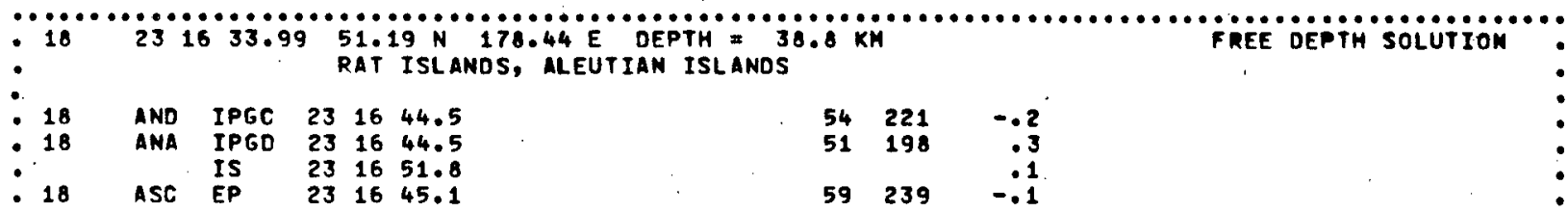




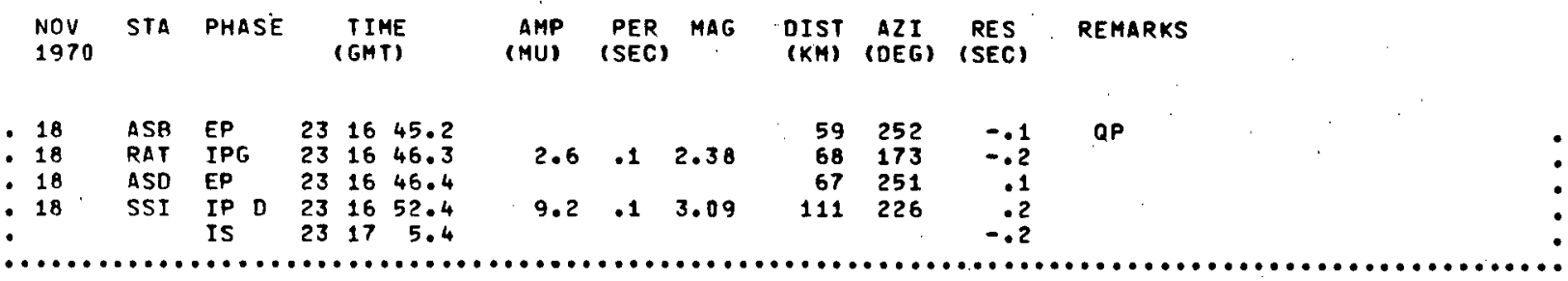

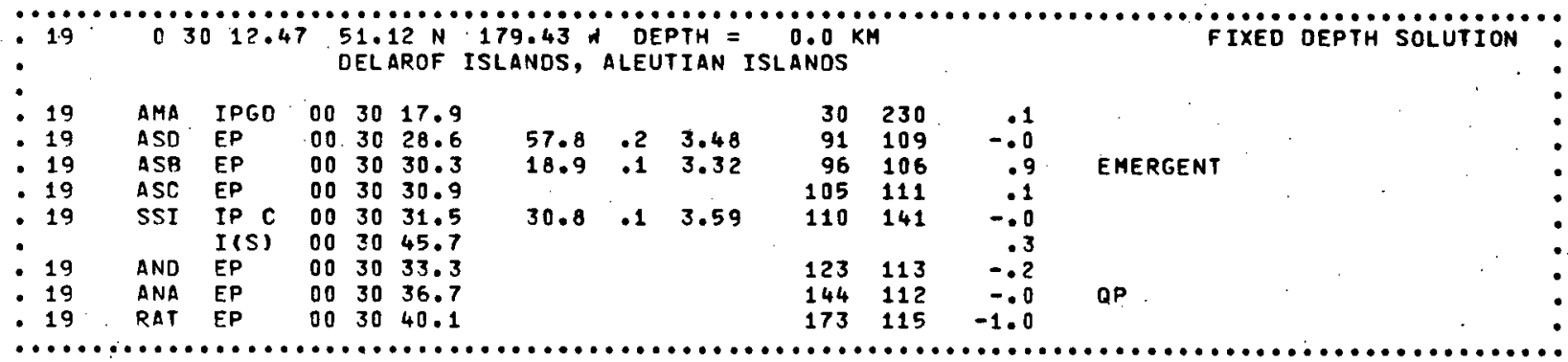

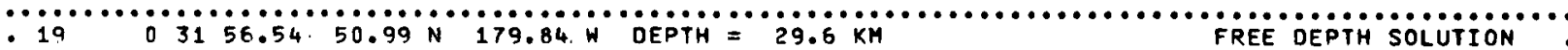

FREE DEPTH SOLUTION

$\begin{array}{lllllllll} & 19 & & \end{array}$

- 19 I(S) 003215.4

- 19 ASB EP $0032 \quad 9.4$

- 19 ASC EP DO 3211.5

- 19 ANO IP OO 3214.4

- 19 SSI EP 003214.5

I(S) 003227.4

- 19 RAT EP O0 3221.1

$\begin{array}{lllll}3.5 & .1 & 2.51 & 73 & 128\end{array}$

$13.5 \quad .2 \quad 2.81$

$\begin{array}{ll}76 & 123 \\ 88 & 127\end{array}$

$\begin{array}{rr}88 & 127 \\ 106 & 127\end{array}$

$\begin{array}{lllll}6.2 \quad .1 & 2.90 \quad 109 & 158\end{array}$

$\cdots$

$\begin{array}{llllll}19 & \text { AMA } & \text { IPGD } & 04 & 57 & 56 \\ 19 & \text { ASD } & \text { IS } & 04 & 57 & 59 \\ 19 & \text { SSI } & 04 & 58 & 11 . \\ 19 & \text { ASB } & \text { EP } & 04 & 58 & 11 . \\ 19 & \text { ASC } & \text { EP } & 58 & 12 \\ 19 & 04 & 58 & 13 .\end{array}$

${ }_{n}^{8.8} .12 .46$

$156 \quad 125$

- O VERY ODD FREO

$-.3$

NICE LOCAL

QP

- 2 Q

.3

-1
-.1
-.1

QP, EMERGENT

$1 \cdot 9 \cdot 1$

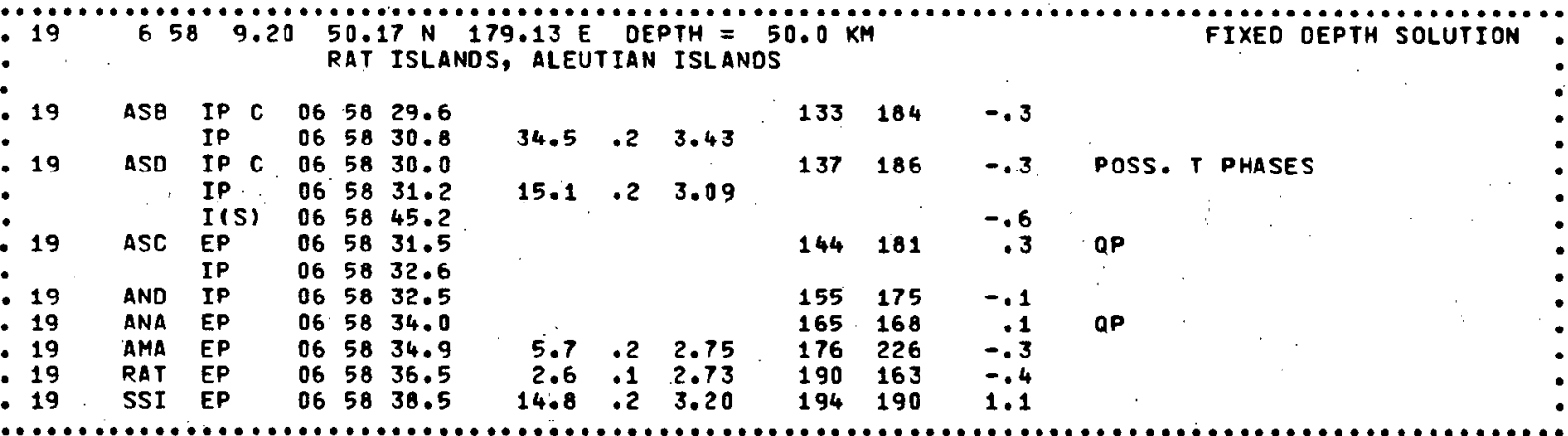

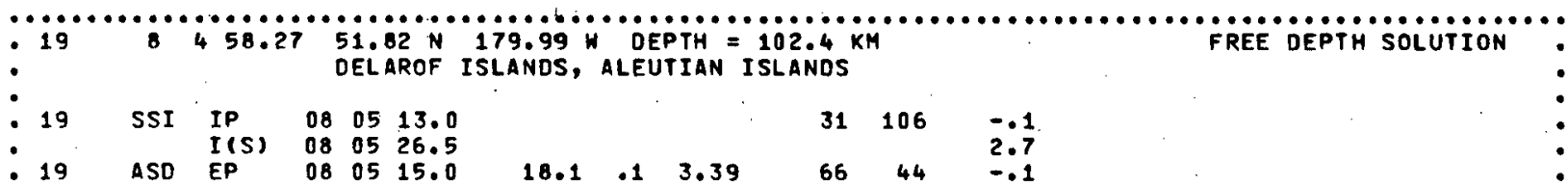




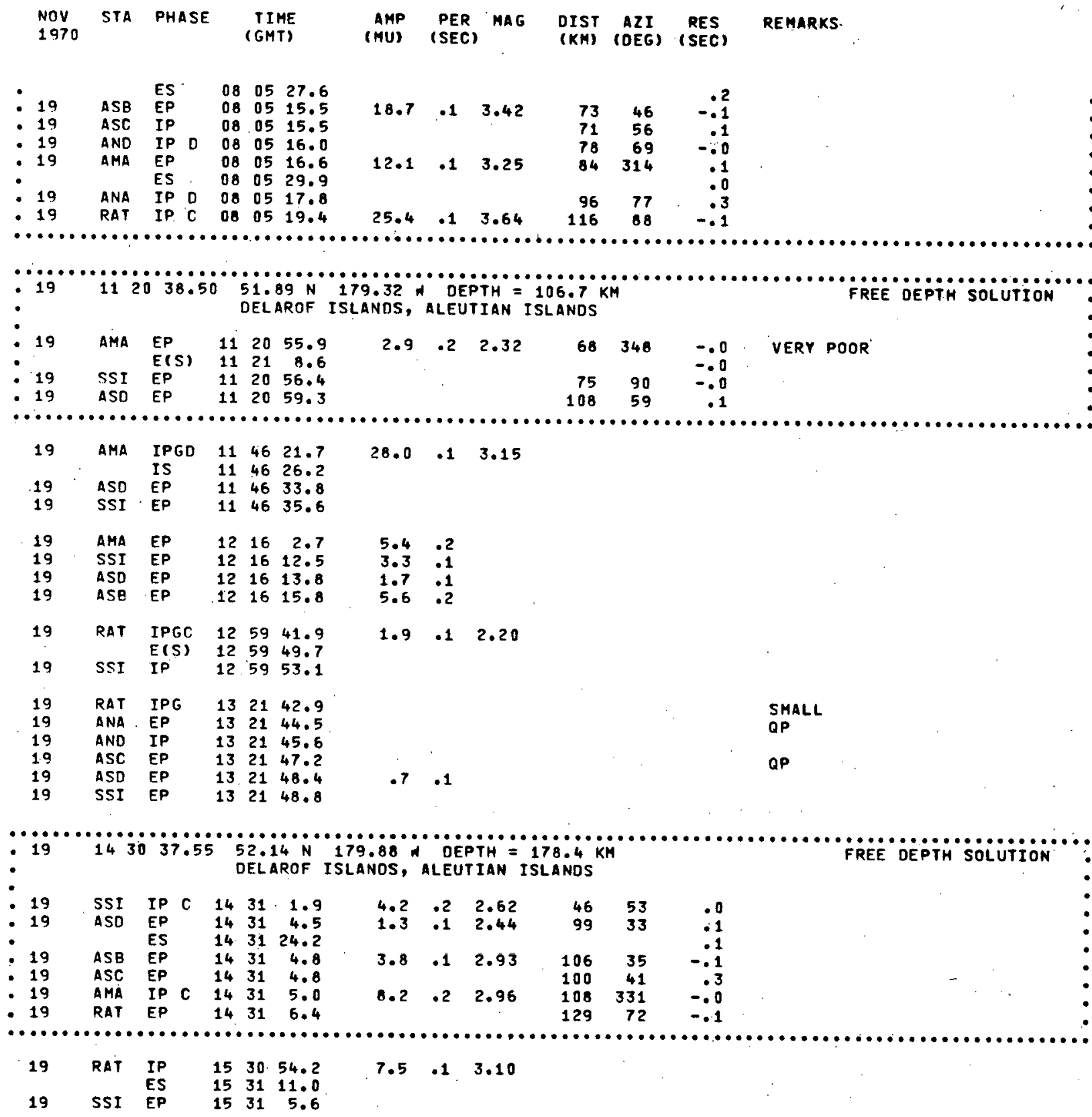

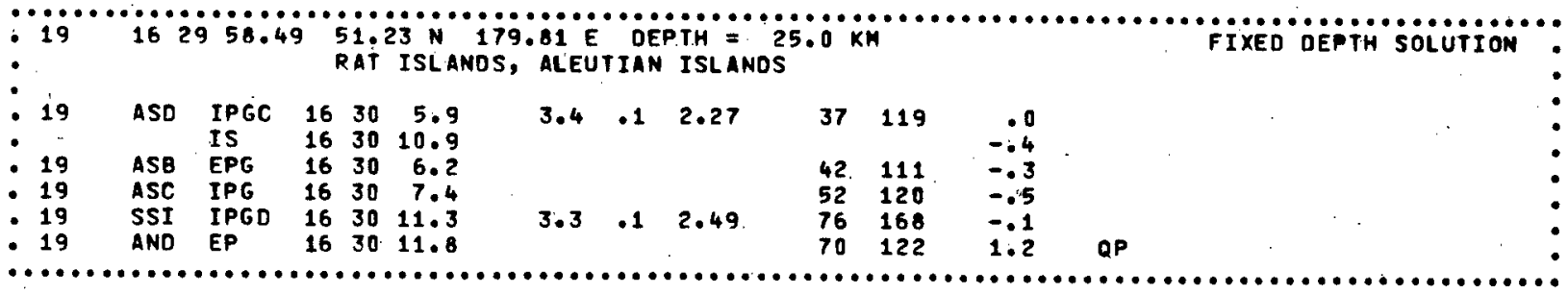




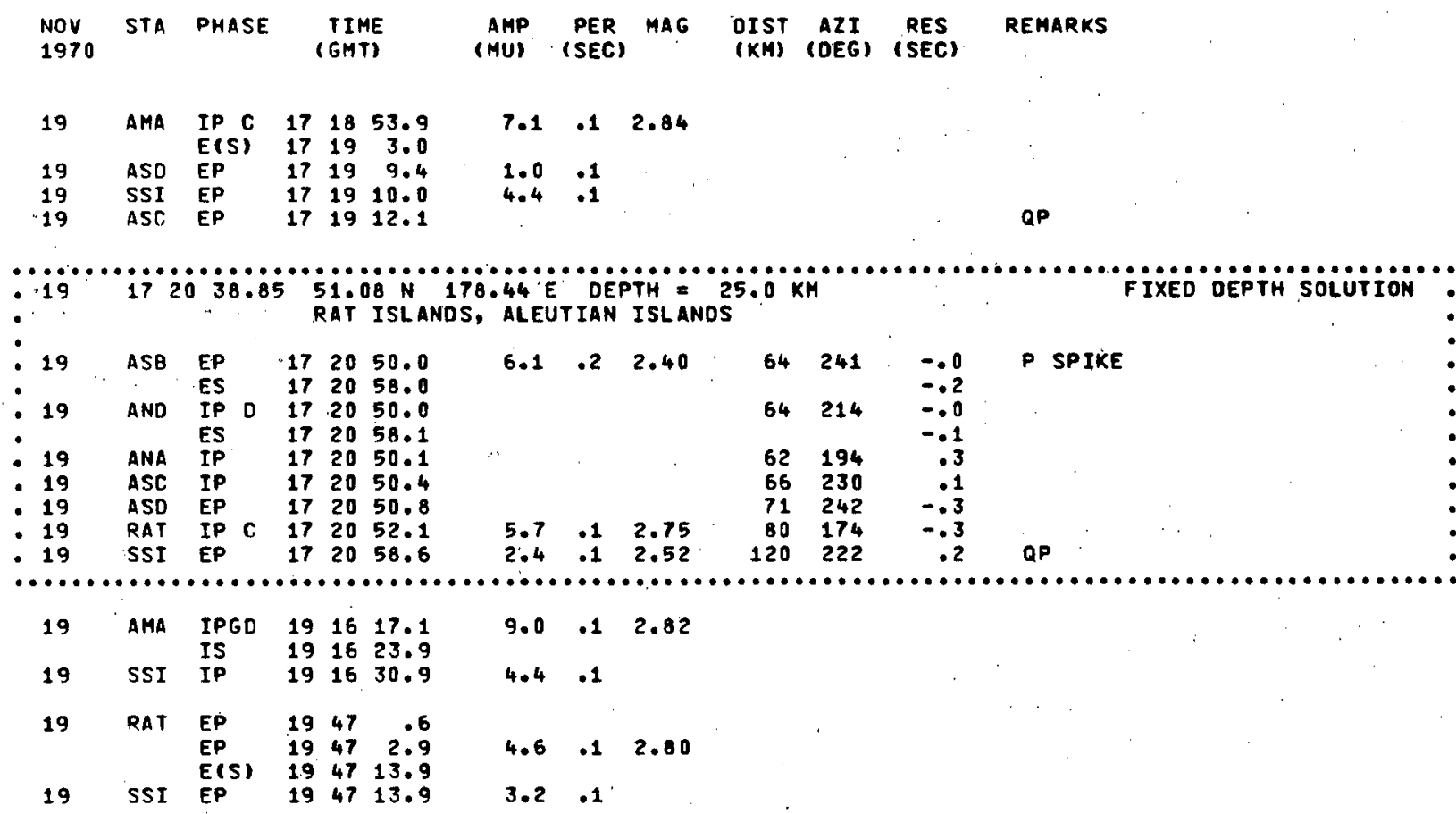

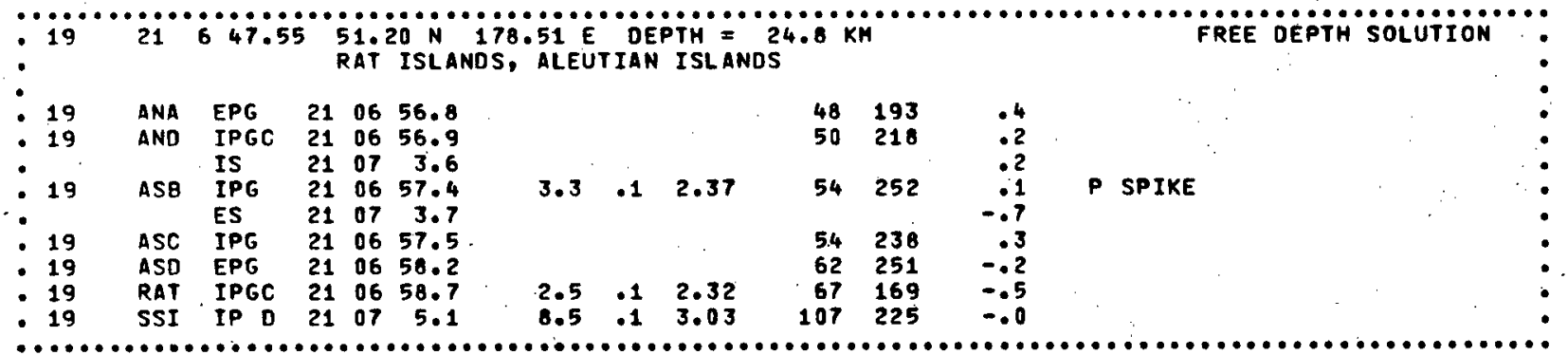

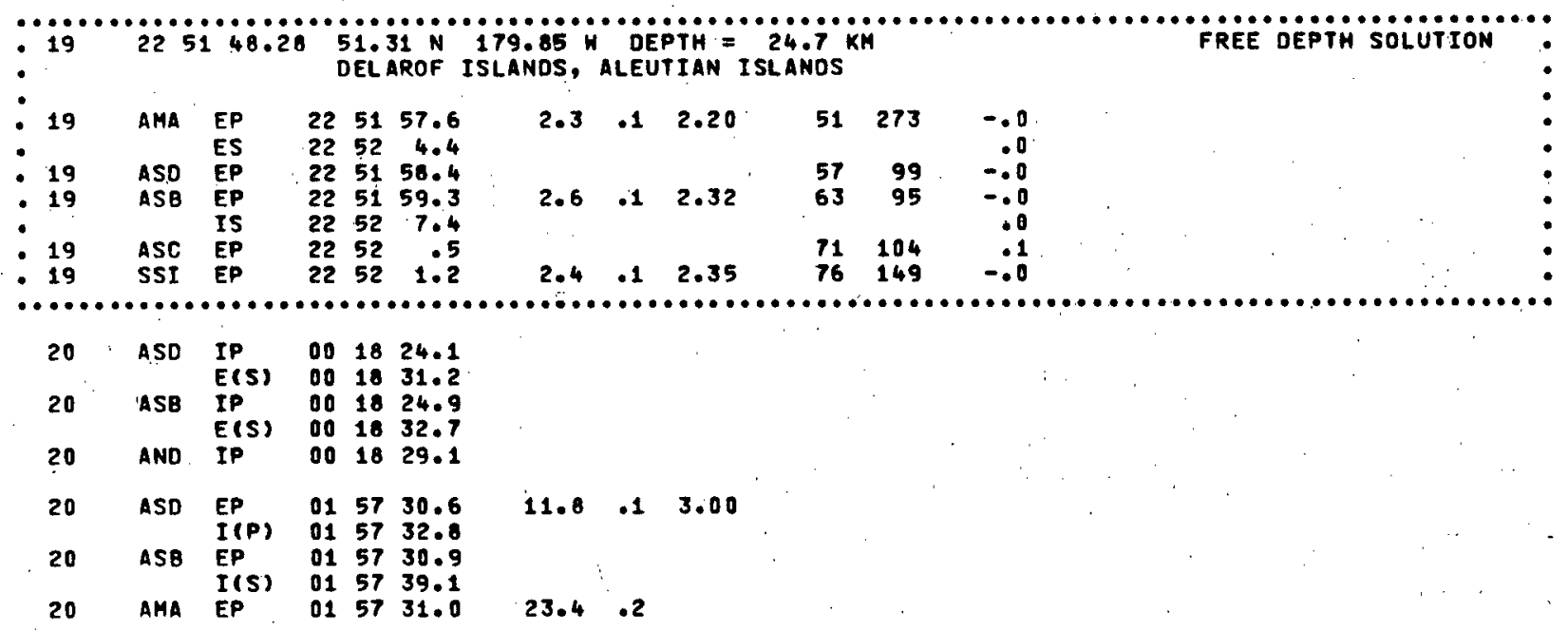




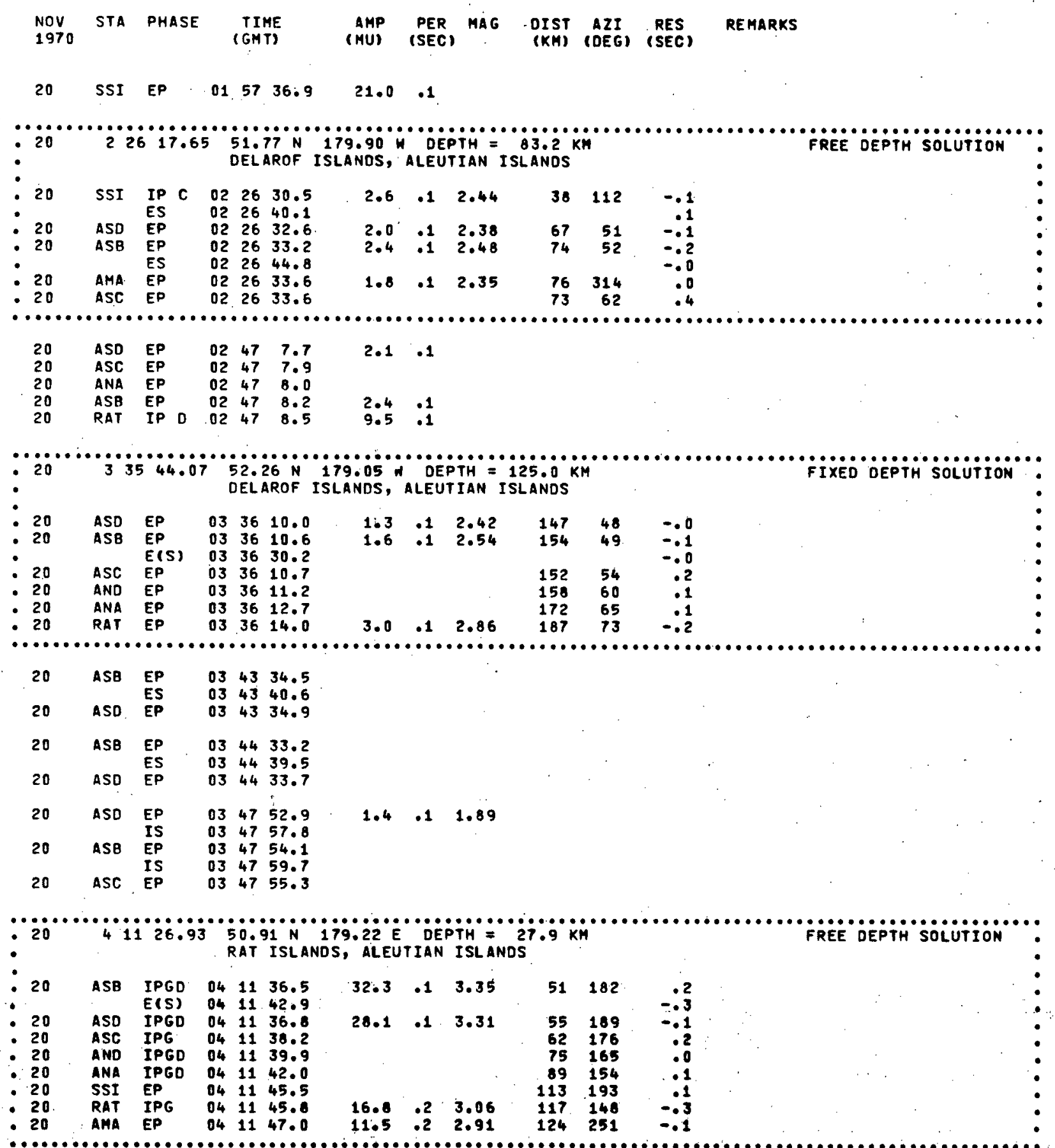




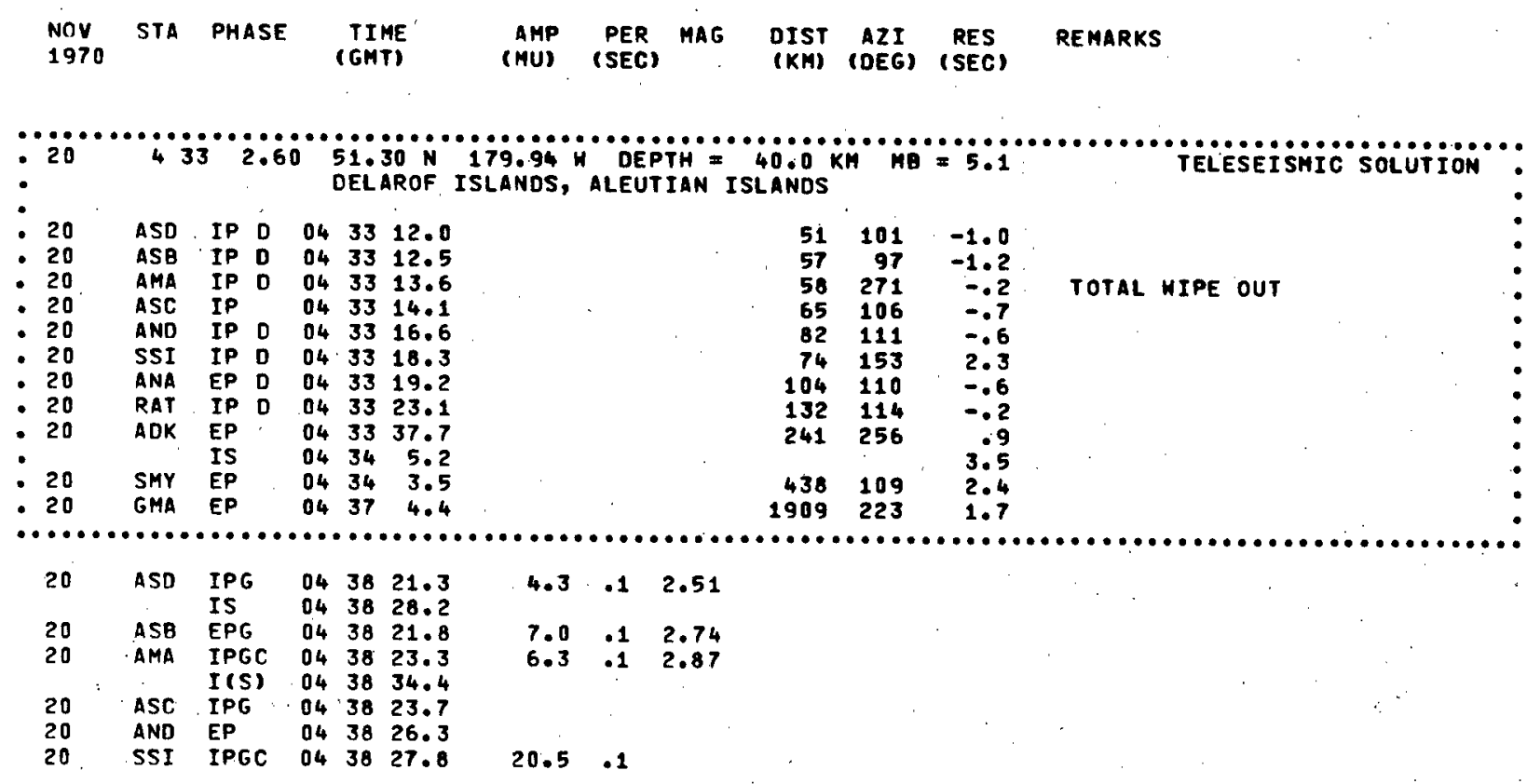

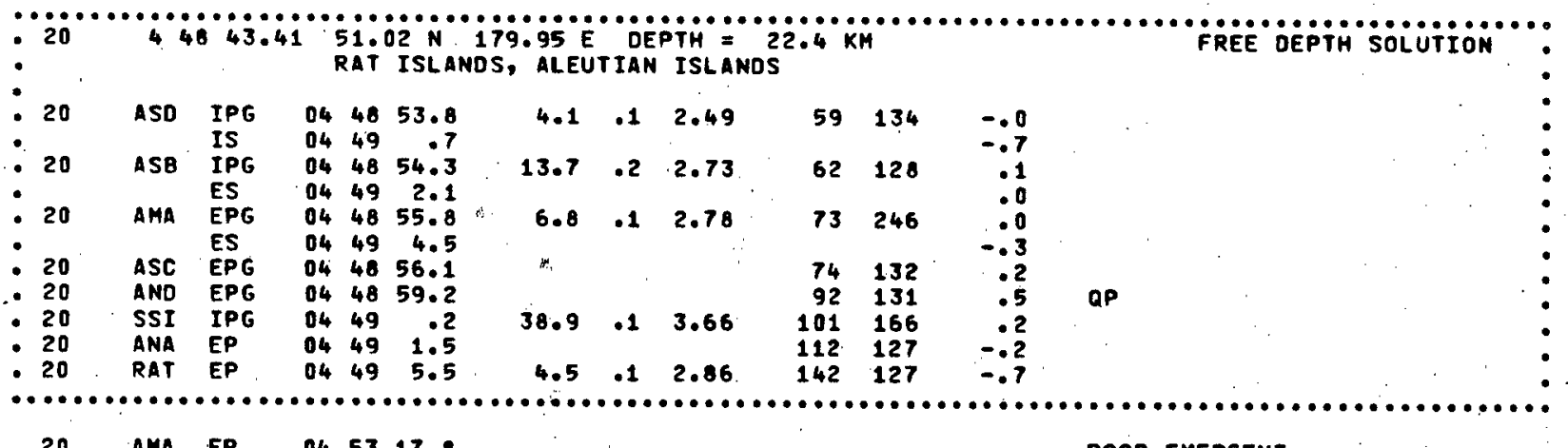

$\begin{array}{llllll}20 & \text { AMA } & \text { EP } & 04 & 53 & 17.8 \\ 20 & \text { SSI } & \text { EP } & 04 & 53 & 28.9 \\ 20 & \text { ASD } & \text { EP } & 04 & 53 & 32.4 \\ 20 & \text { ASC } & \text { EP } & 04 & 53 & 34.0 \\ 20 & \text { AND } & \text { EP } & 04 & 53 & 35.1 \\ 20 & \text { ANA } & \text { EP } & 04 & 53 & 37.8 \\ 20 & \text { RAT } & \text { EP } & 04 & 53 & 40.2 \\ 20 & \text { ANA } & \text { IPG } & 05 & 07 & 11.6 \\ & & \text { IS } & 05 & 07 & 15.0 \\ 20 & \text { RAT } & \text { IPG } & 05 & 07 & 12.0\end{array}$

POOR, EMERGENT

HEST ENO LOCAL

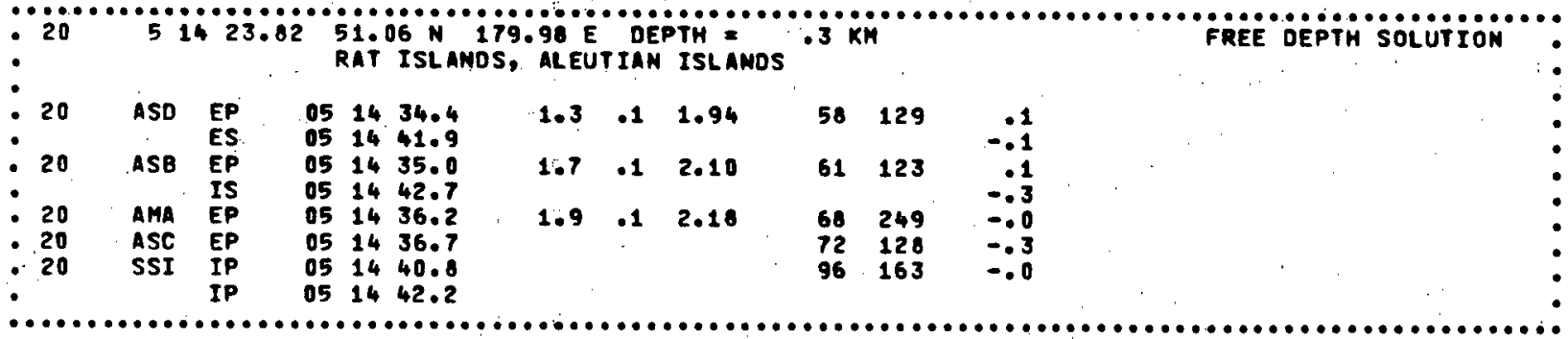




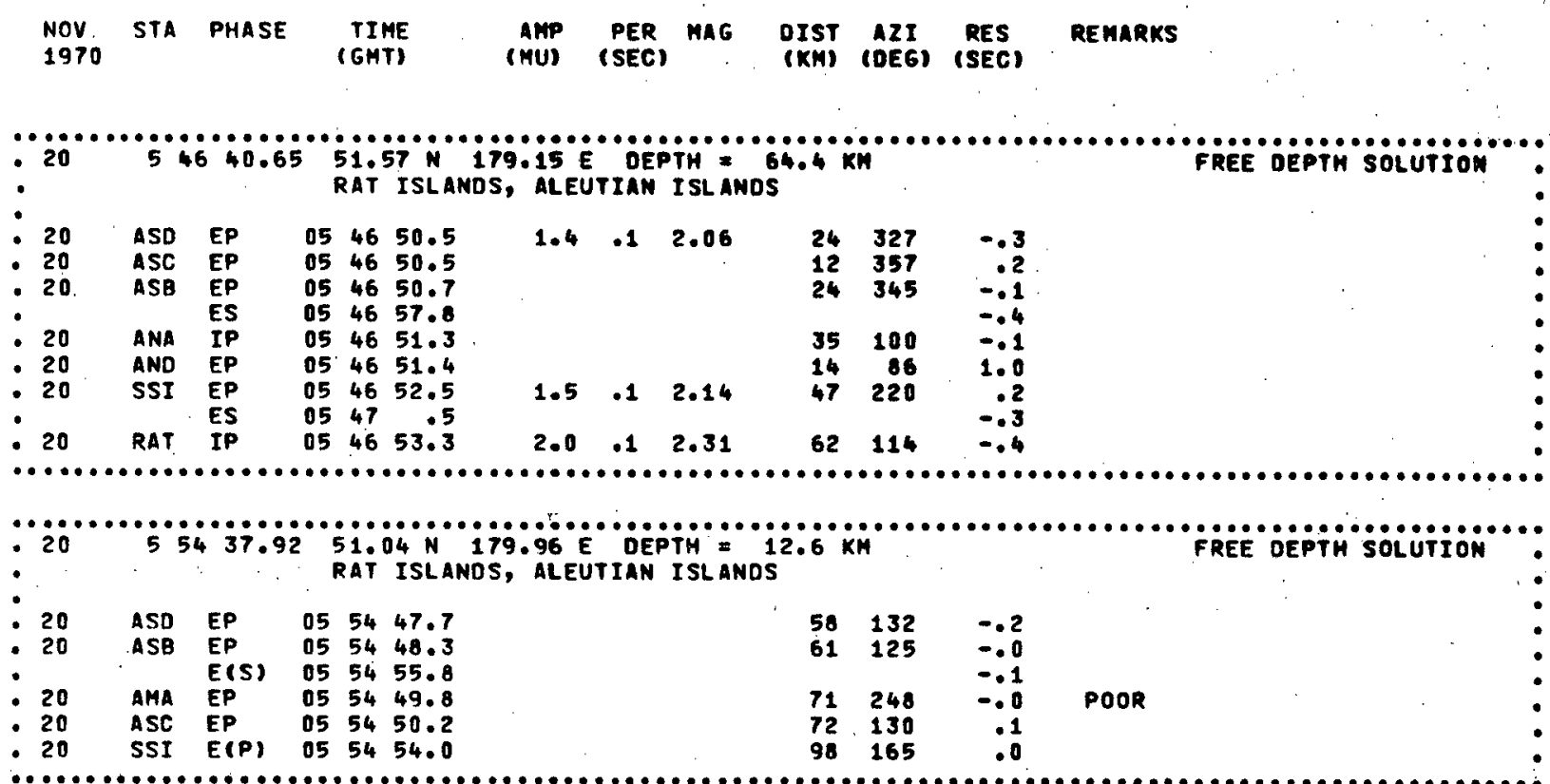

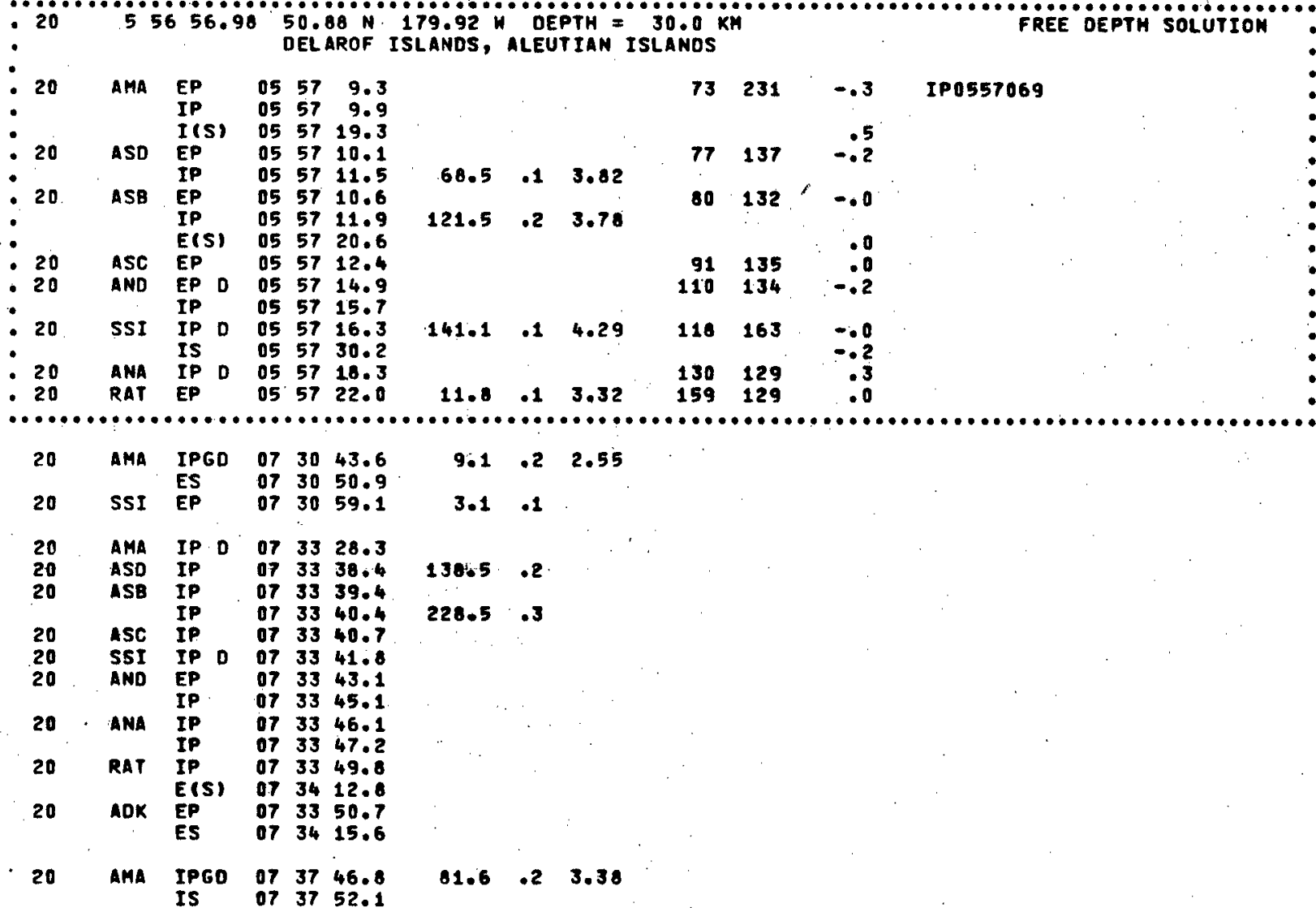




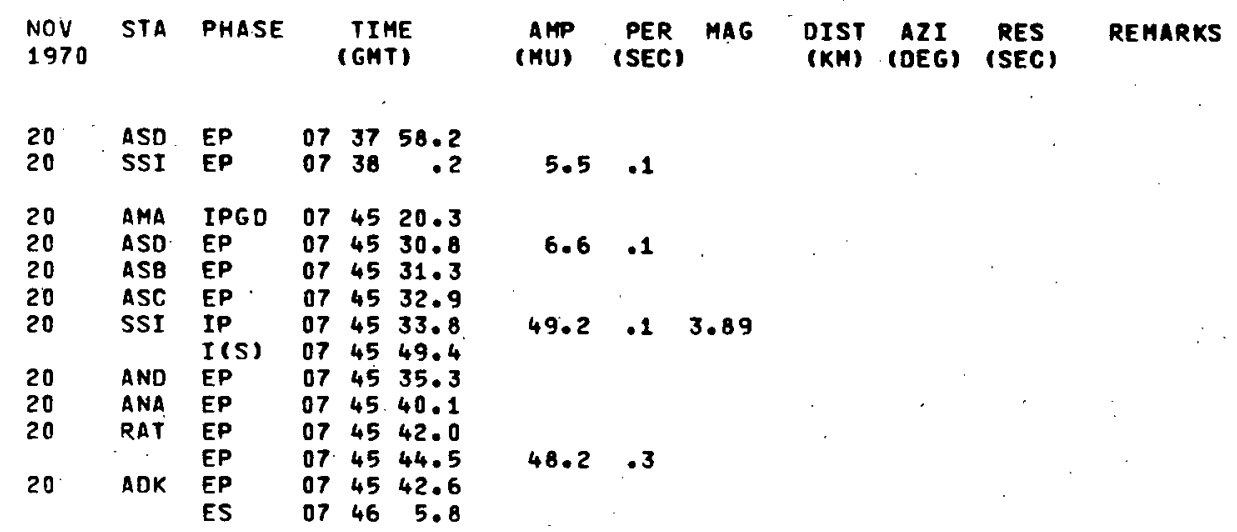

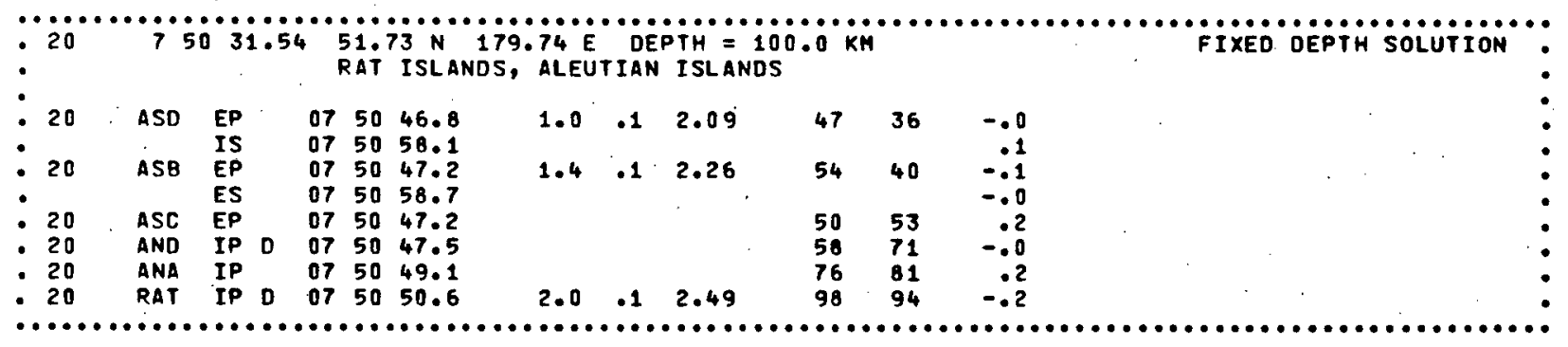

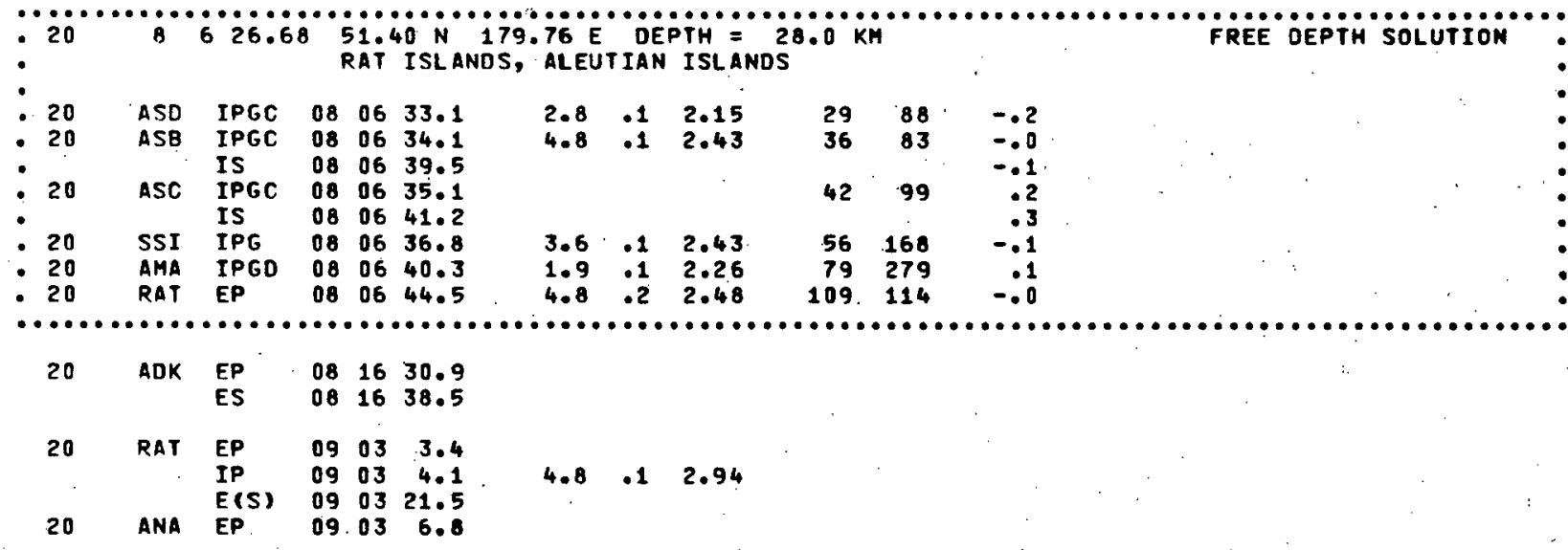

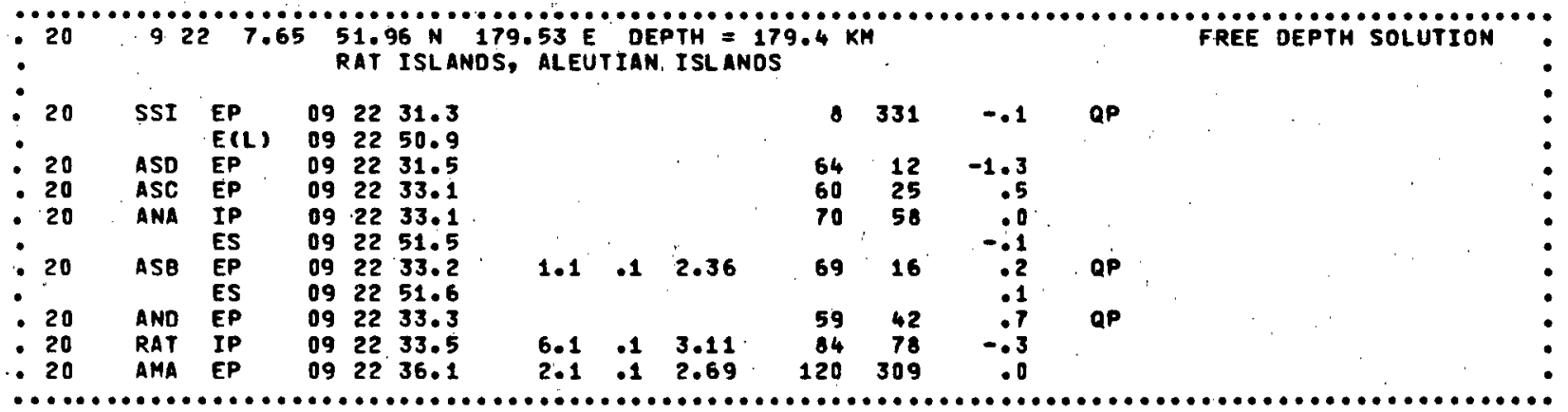




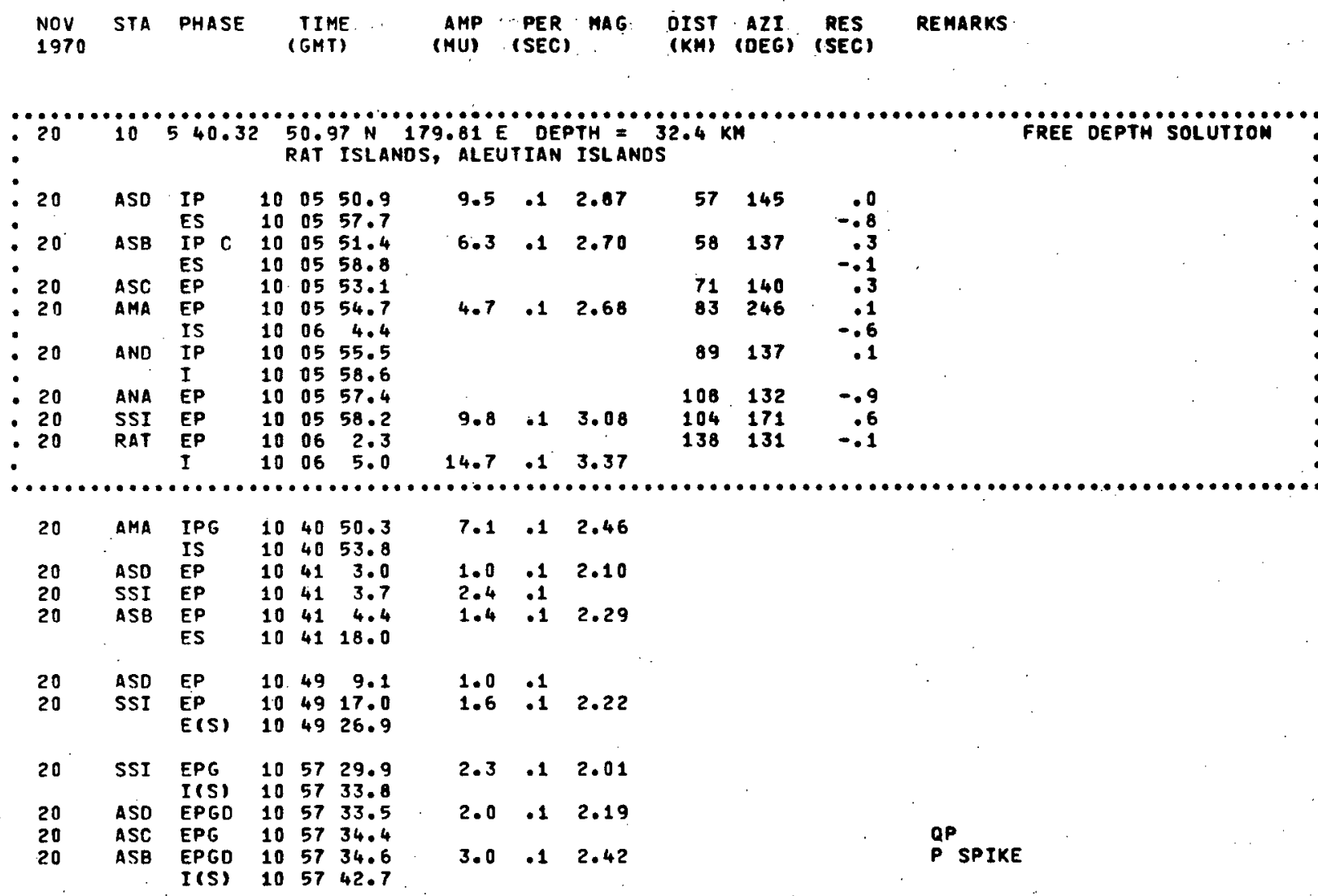

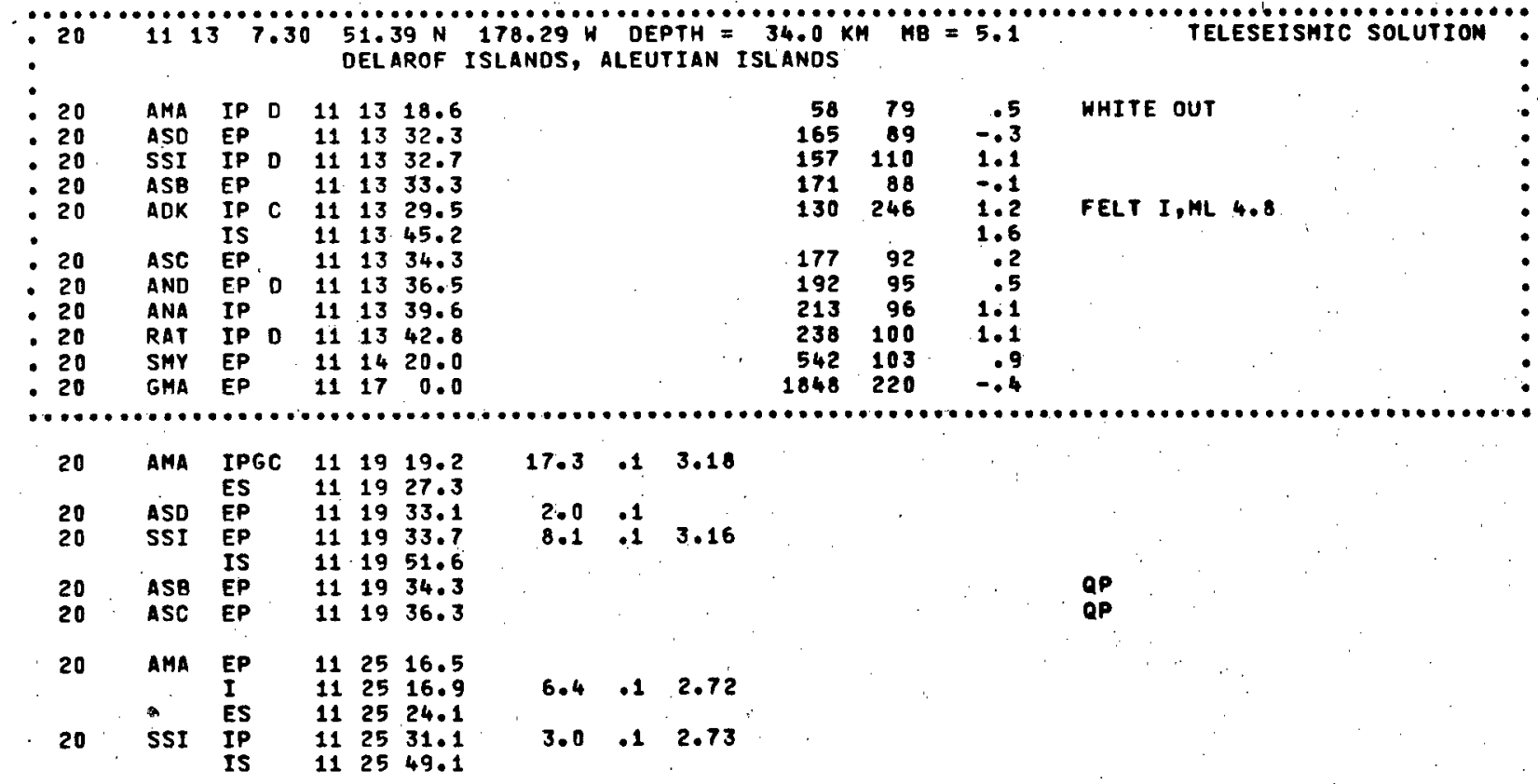




\begin{tabular}{|c|c|c|c|c|c|c|c|c|c|c|c|c|}
\hline \multirow{2}{*}{$\begin{array}{l}\text { NoV } \\
1970 \\
20 \\
20 \\
20\end{array}$} & \multirow{2}{*}{$\begin{array}{l}\text { STA } \\
\text { AMA } \\
\text { ASD } \\
\text { SSI }\end{array}$} & $\begin{array}{l}\text { PHASE } \\
\text { IPGD }\end{array}$ & \multicolumn{3}{|c|}{$\begin{array}{c}\text { TIME } \\
\text { (GMT) }\end{array}$} & $\begin{array}{l}\text { AMP } \\
\text { (MU) } \\
120.7\end{array}$ & $\begin{array}{l}\text { PER } \\
\text { (SEC) } \\
.1\end{array}$ & MAG & $\begin{array}{l}\text { DIST } \\
\text { (KM) }\end{array}$ & $\begin{array}{c}\text { AZI } \\
\text { (DEG) }\end{array}$ & $\begin{array}{l}\text { RES } \\
\text { (SEC) }\end{array}$ & $\begin{array}{l}\text { RE.MARKS } \\
\text { MIN.AMPLITUDE }\end{array}$ \\
\hline & & $\begin{array}{l}\text { IPGD } \\
\text { IP } 0 \\
\text { IP } \\
\text { ES }\end{array}$ & $\begin{array}{l}11 \\
11 \\
11 \\
11\end{array}$ & $\begin{array}{l}27 \\
28 \\
28 \\
28\end{array}$ & $\begin{array}{r}54.0 \\
7.6 \\
0.3 \\
28.6\end{array}$ & $\begin{array}{r}120.7 \\
19.3 \\
58.6\end{array}$ & $\begin{array}{l}.1 \\
.1 \\
.1\end{array}$ & $\begin{array}{l}3.58 \\
4.07\end{array}$ & & & & \\
\hline 20 & ASB & $\begin{array}{l}E P \\
E S\end{array}$ & $\begin{array}{l}11 \\
11\end{array}$ & $\begin{array}{l}28 \\
28\end{array}$ & $\begin{array}{r}8.7 \\
29.2\end{array}$ & 46.9 & .2 & 3.68 & & & & \\
\hline 20 & $\triangle D K$ & $\begin{array}{l}\text { EP } \\
\text { ES }\end{array}$ & $\begin{array}{l}11 \\
11\end{array}$ & $\begin{array}{l}28 \\
28\end{array}$ & $\begin{array}{r}4.7 \\
20.4\end{array}$ & & & & & & & \\
\hline $\begin{array}{l}20 \\
20 \\
20 \\
20\end{array}$ & $\begin{array}{l}\text { ASC } \\
\text { AND } \\
\text { ANA } \\
\text { RAT }\end{array}$ & $\begin{array}{l}E P \\
E P \\
E P \\
E P\end{array}$ & $\begin{array}{l}11 \\
11 \\
11\end{array}$ & $\begin{array}{l}28 \\
28 \\
28 \\
28\end{array}$ & $\begin{array}{r}9.6 \\
11.9 \\
15.2 \\
18.4\end{array}$ & & & & & & & \\
\hline & RAT & EP & 11 & 28 & 18.4 & 34.7 & $\cdot 2$ & & & & & \\
\hline 20 & $A S B$ & $\begin{array}{l}\text { EP } \\
\text { ES }\end{array}$ & $\begin{array}{l}11 \\
11\end{array}$ & $\begin{array}{l}43 \\
43\end{array}$ & $\begin{array}{l}35.2 \\
52.9\end{array}$ & 2.3 & .1 & 2.61 & & . & & \\
\hline 20 & ASC & EP & 11 & 43 & 35.2 & & & & & & & \\
\hline $\begin{array}{l}20 \\
20\end{array}$ & $\begin{array}{l}\text { ASD } \\
\text { SSI. }\end{array}$ & $\begin{array}{l}\text { EP } \\
\text { EP }\end{array}$ & 11 & 43 & 35.6 & 1.7 & 1 & 2.48 & & & . & OP \\
\hline & & & & & & & & & & & & \\
\hline 20 & AMA & $\begin{array}{l}\text { IP } \\
\text { IP }\end{array}$ & 12. & 09 & 50.3 & & & & & & & \\
\hline 20 & SSI & IP & 12 & 10 & 4.7 & 3.6 & .1 & 2.82 & & & & \\
\hline & & ES & 12 & 10 & 23.2 & & & & & & & \\
\hline
\end{tabular}

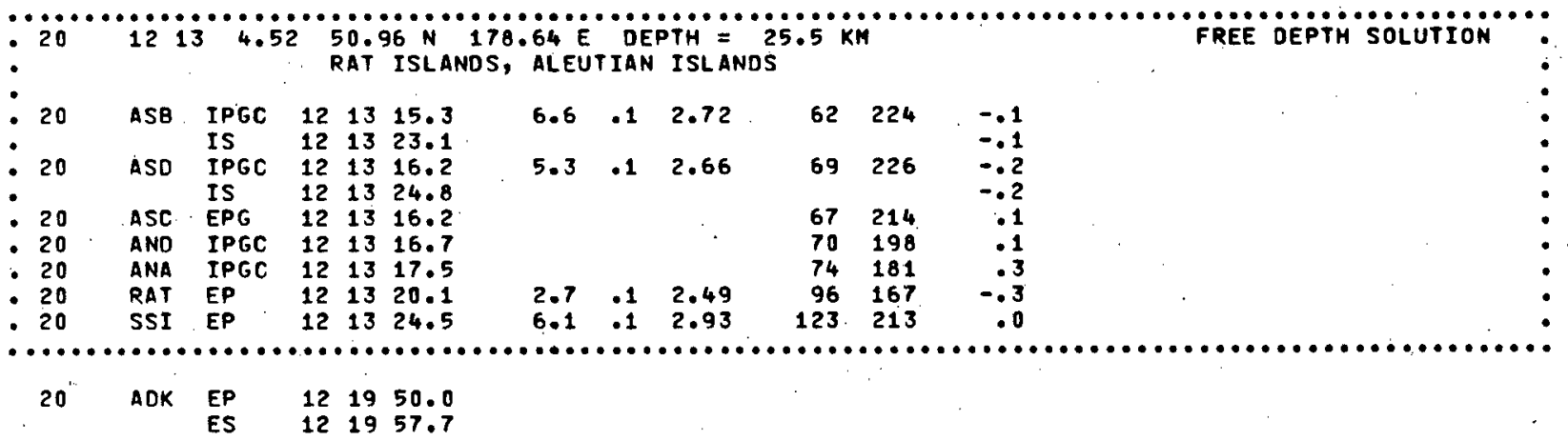

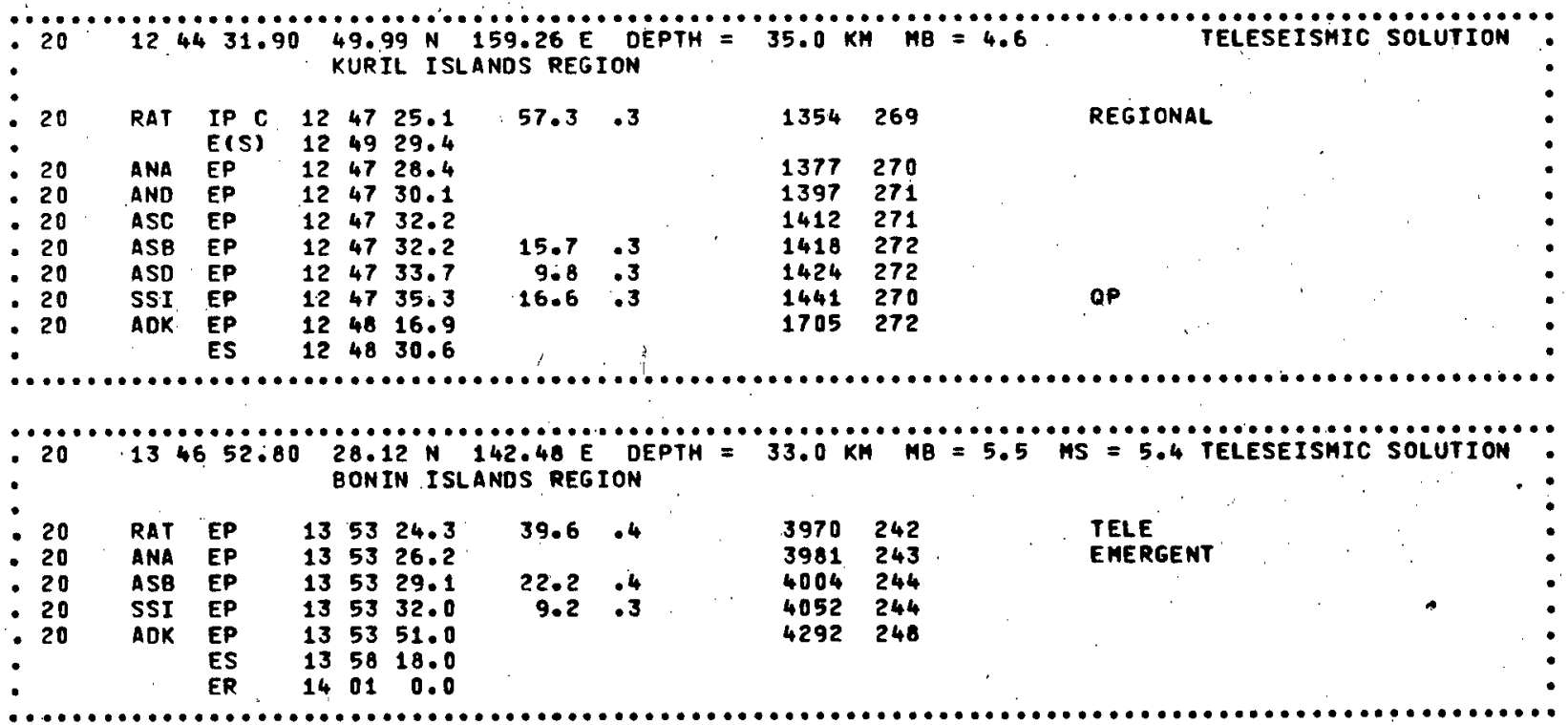




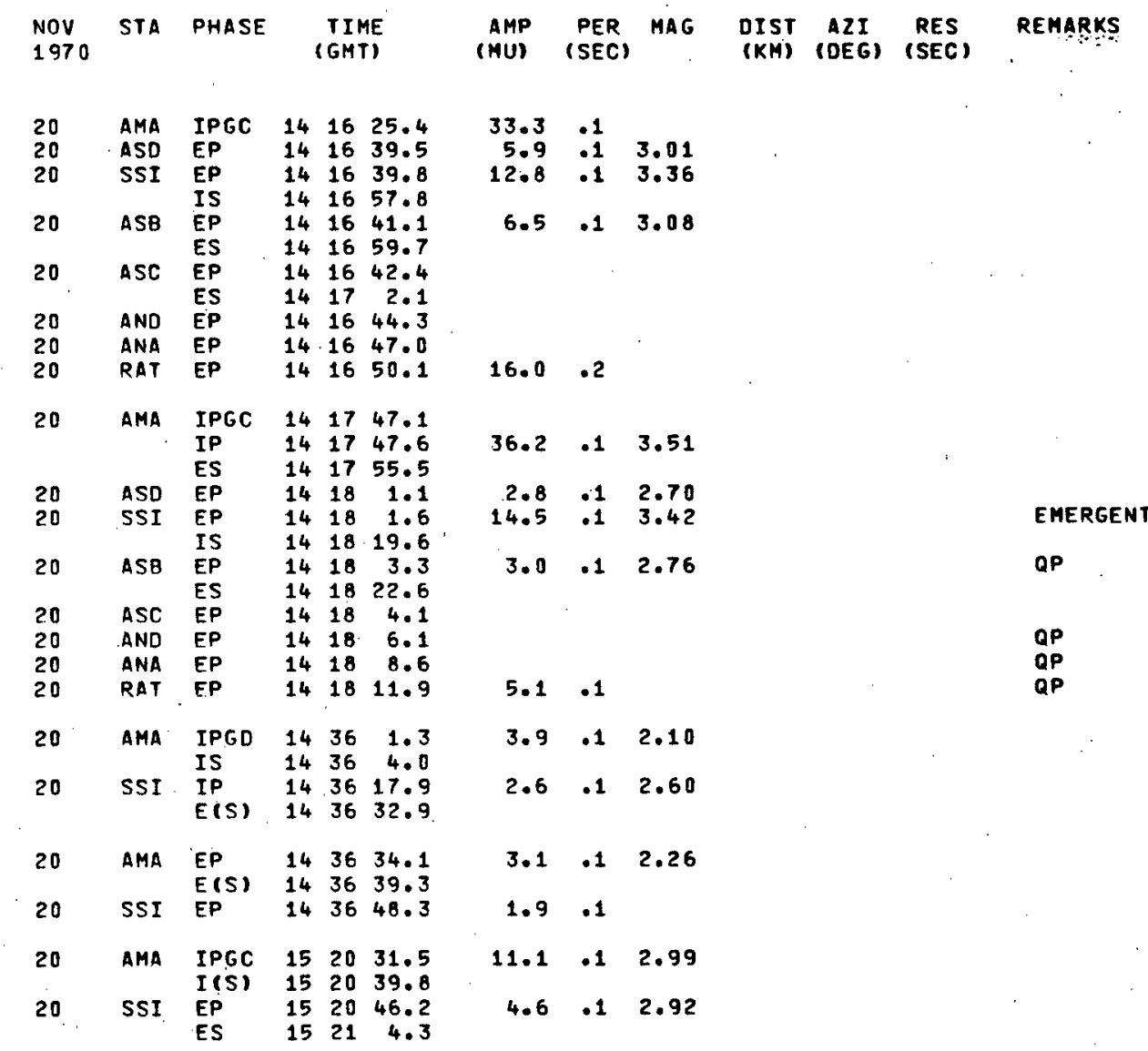

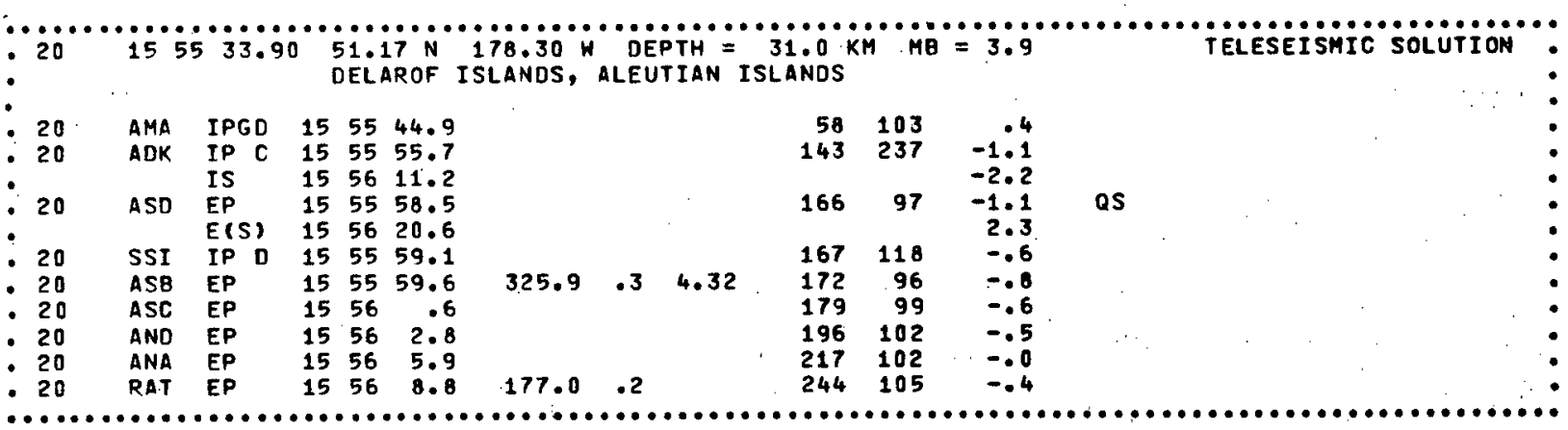

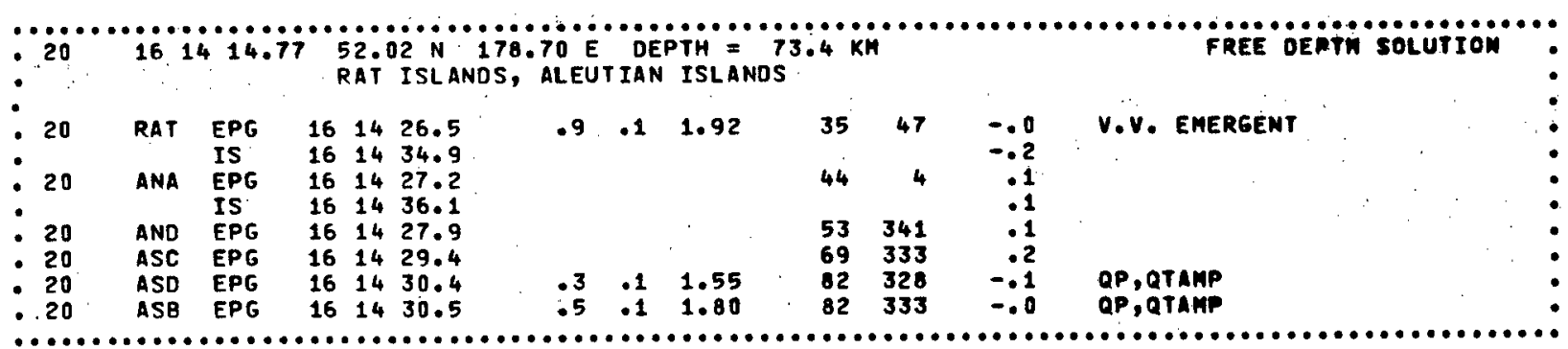




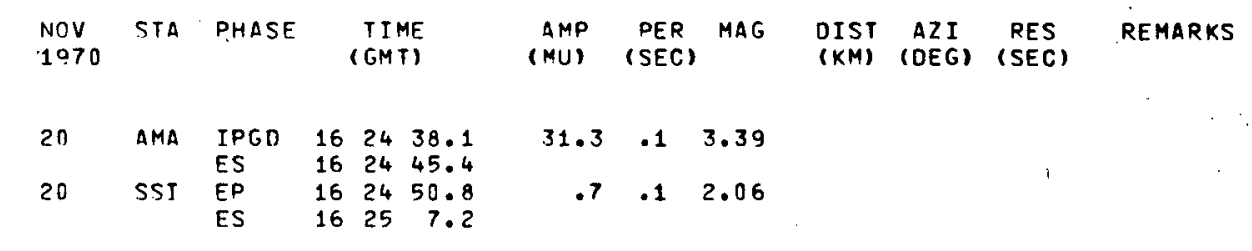

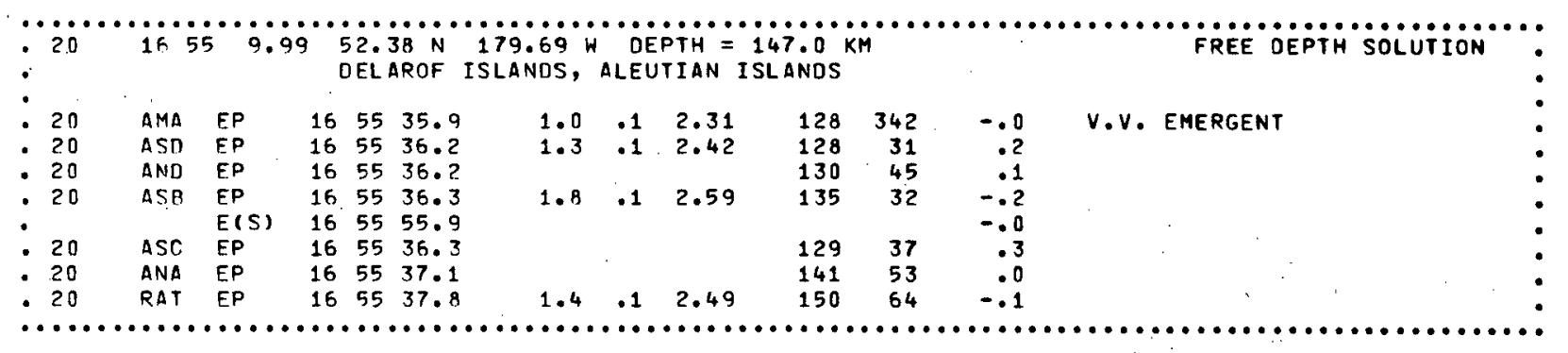

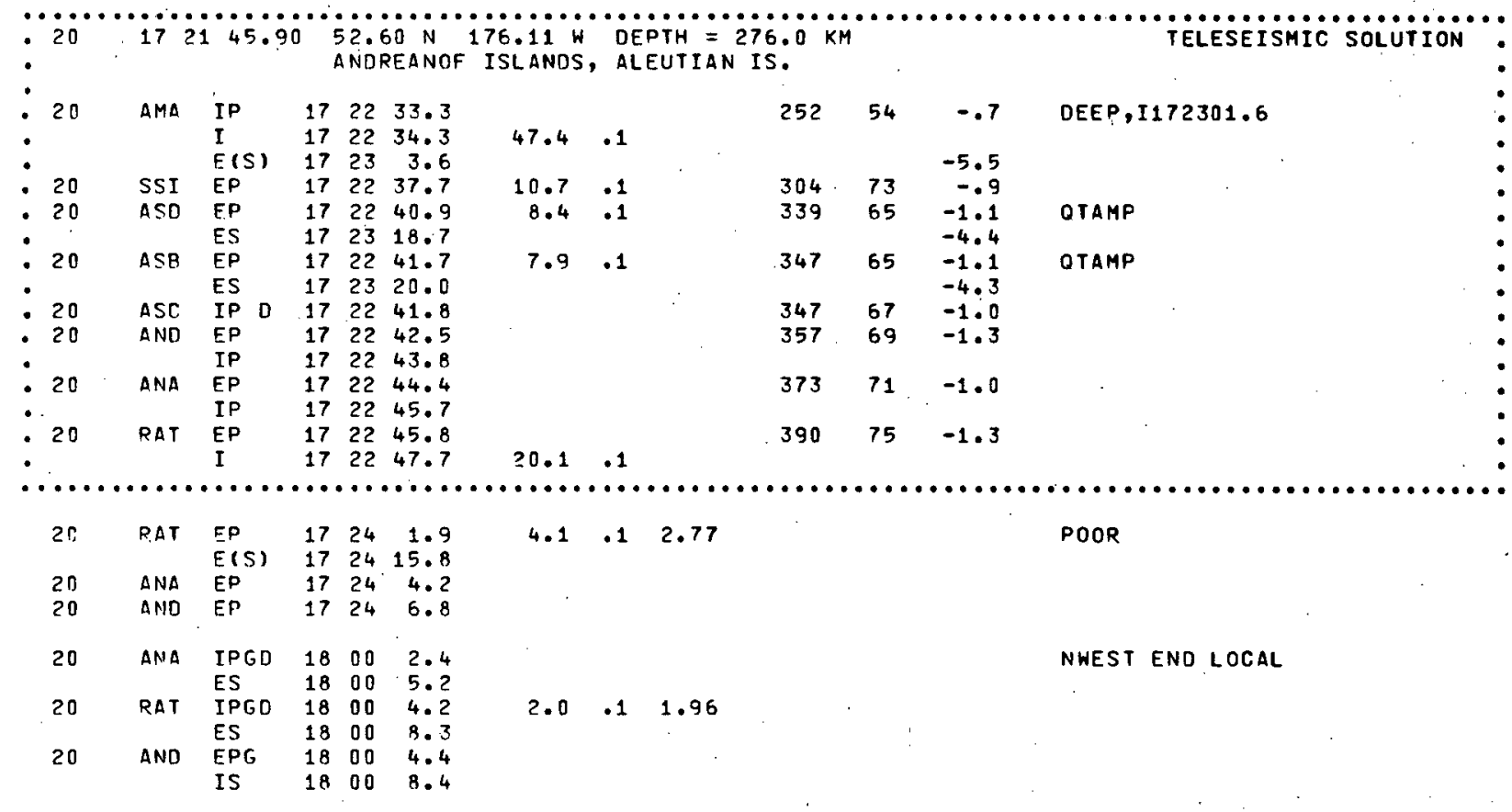

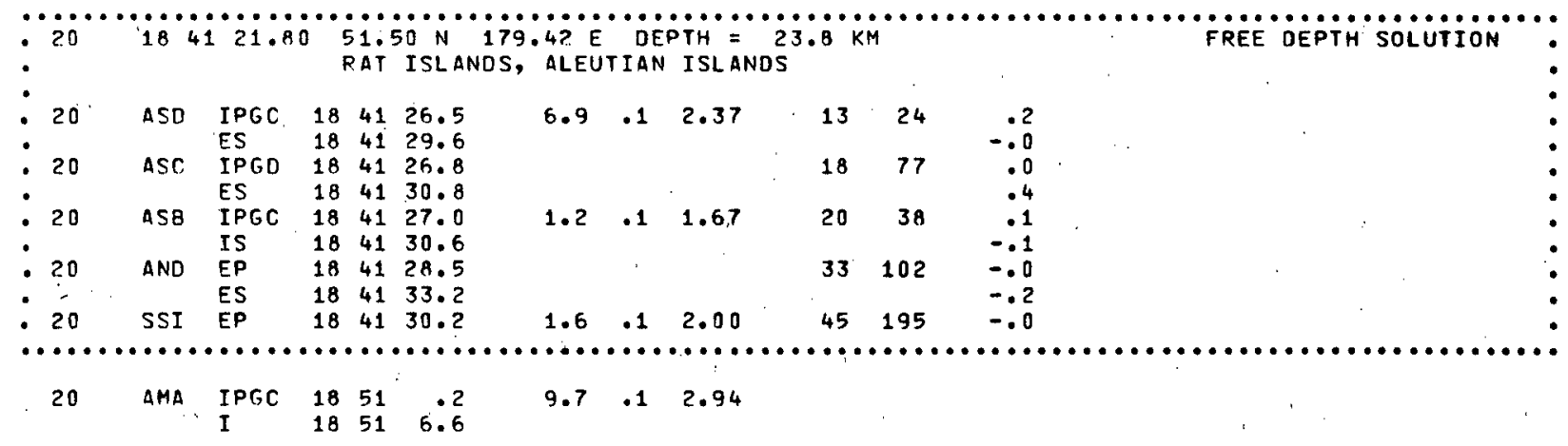




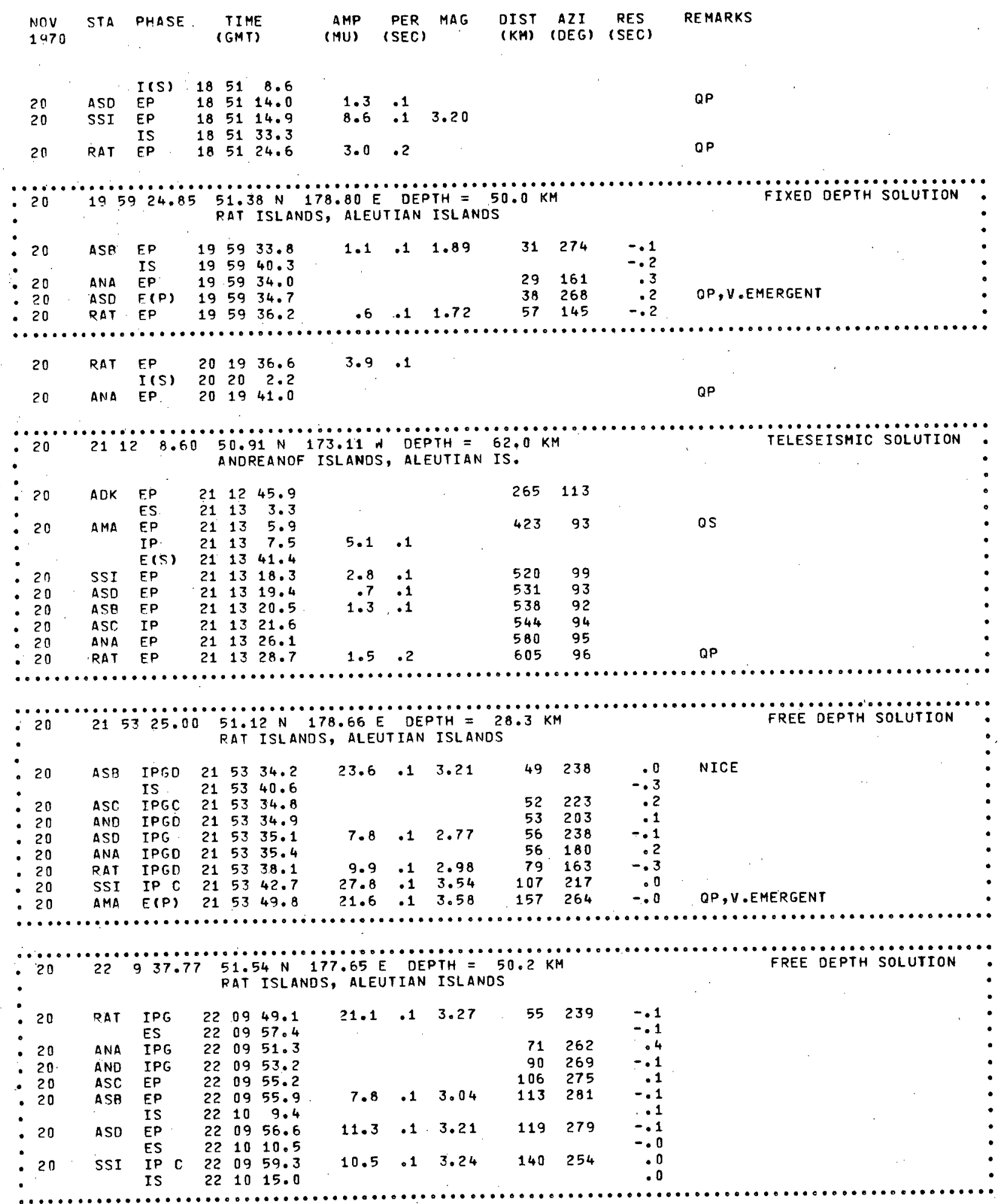




NOV STA PHASE TIME
1970

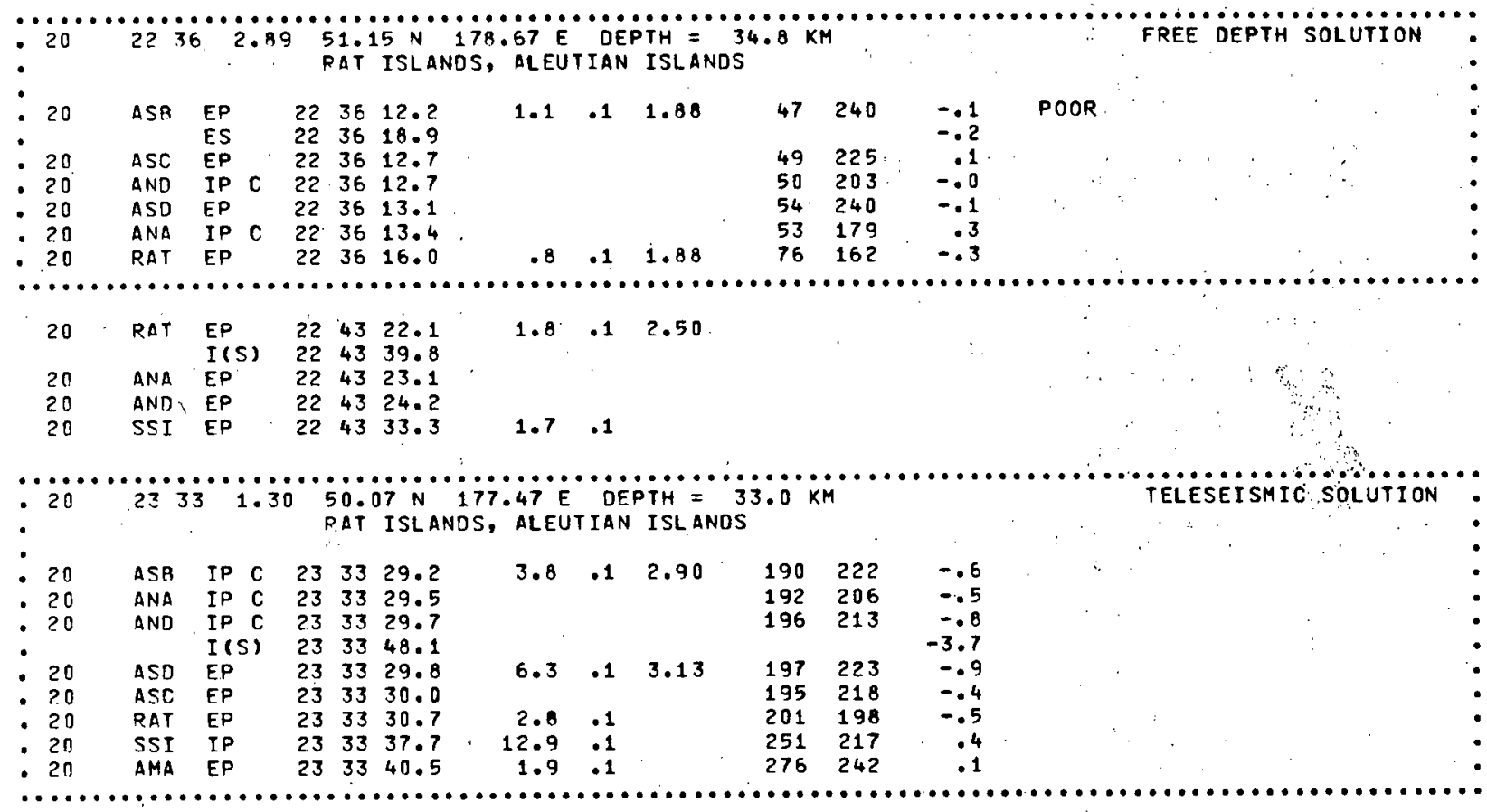

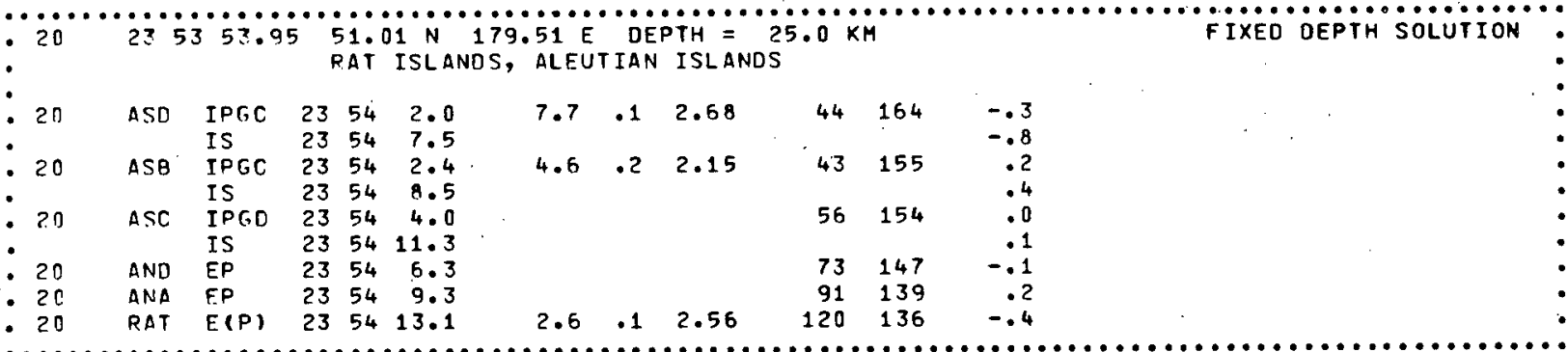

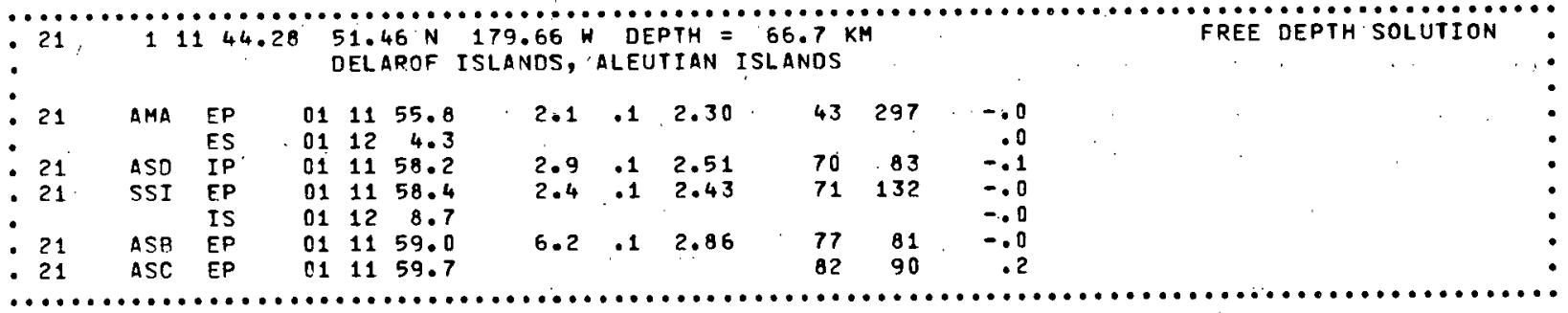

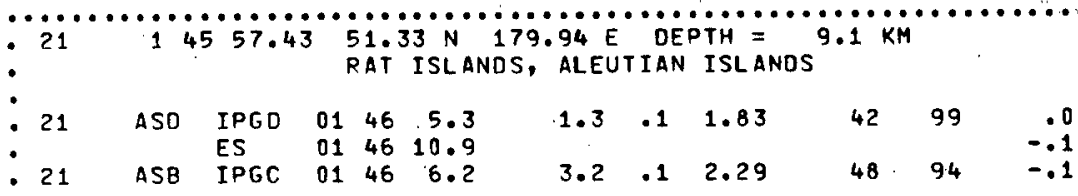




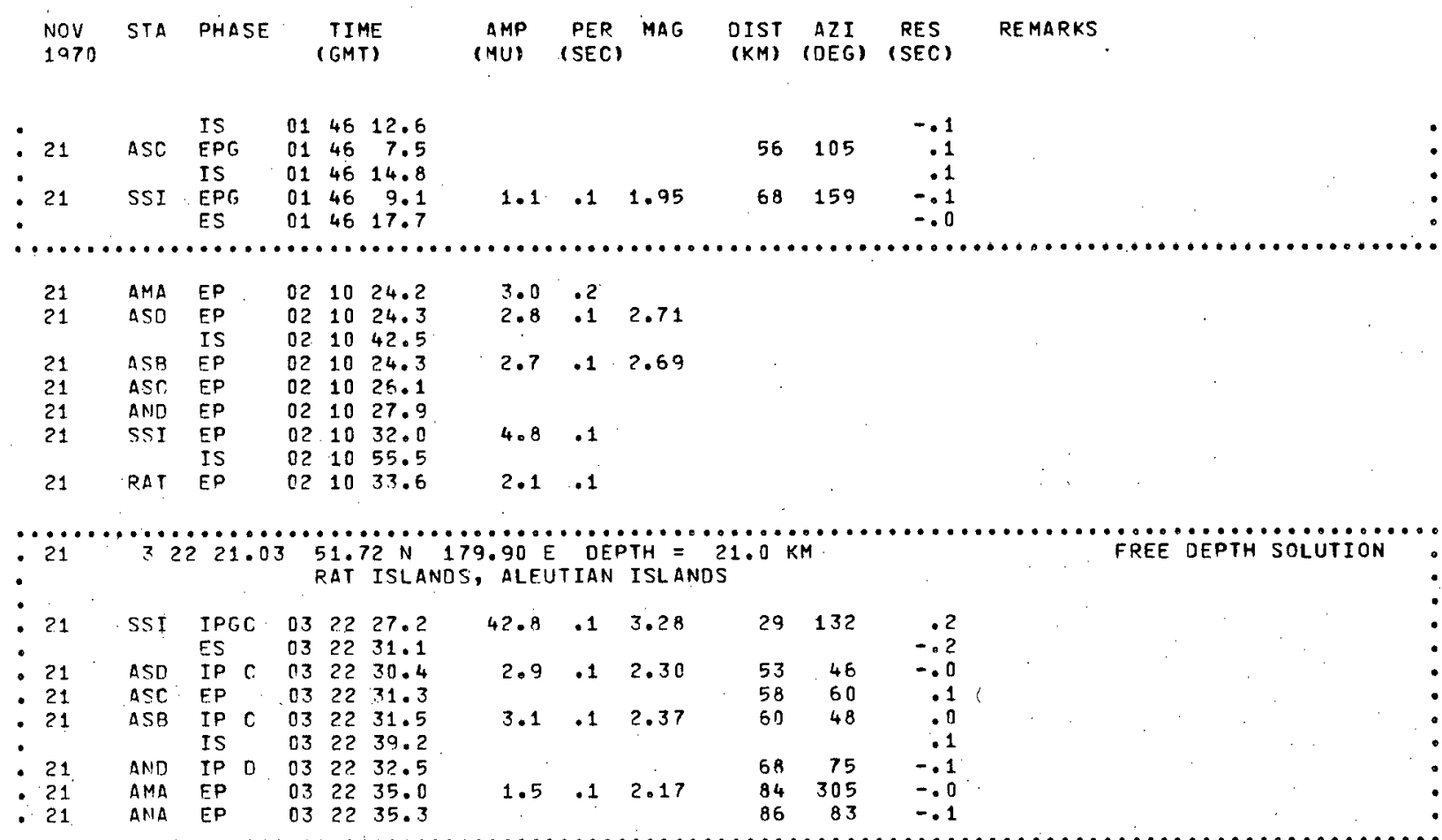

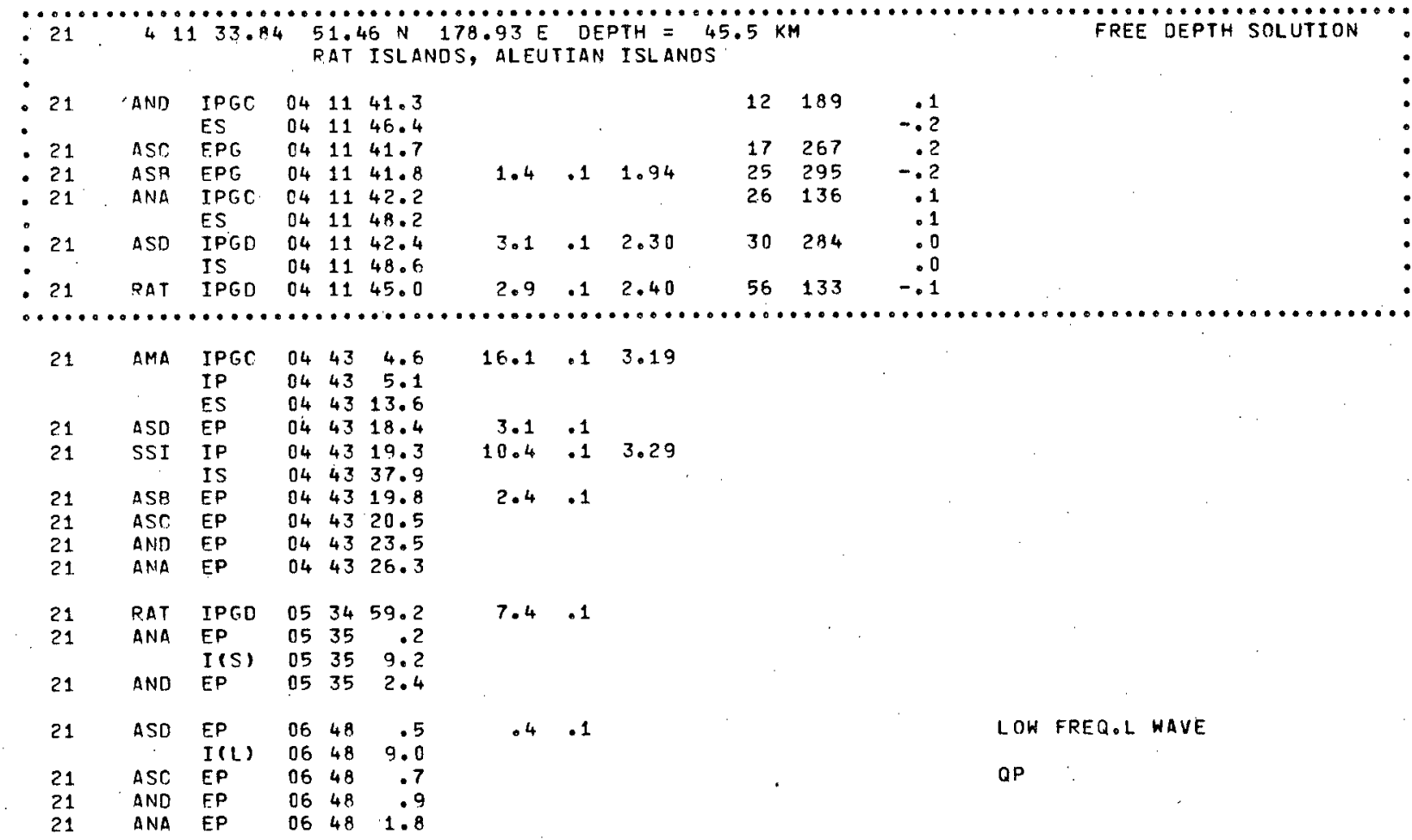




\begin{tabular}{|c|c|c|c|c|c|c|c|c|}
\hline $\begin{array}{l}\text { NOV } \\
1970\end{array}$ & STA & PHASE & & $\begin{array}{l}\text { TIM } \\
\text { GMT }\end{array}$ & & $\begin{array}{l}\text { AMP } \\
\text { (MU) }\end{array}$ & $\begin{array}{c}\text { PER } \\
\text { (SEC) }\end{array}$ & MAG \\
\hline 21 & SSI & $\begin{array}{l}\text { IPGD } \\
\text { IS }\end{array}$ & $\begin{array}{l}07 \\
07\end{array}$ & $\begin{array}{l}20 \\
20\end{array}$ & $\begin{array}{l}22.9 \\
27.7\end{array}$ & 3.0 & .1 & 2.21 \\
\hline $\begin{array}{l}21 \\
21\end{array}$ & $\begin{array}{l}\text { ASD } \\
\text { ASB }\end{array}$ & $\begin{array}{l}\text { EPG } \\
\text { IPGD } \\
\text { ES }\end{array}$ & $\begin{array}{l}07 \\
07 \\
07\end{array}$ & $\begin{array}{l}20 \\
20 \\
20\end{array}$ & $\begin{array}{l}23.7 \\
24.8 \\
30.7\end{array}$ & $\begin{array}{l}1.1 \\
1.1\end{array}$ & .1 & $\begin{array}{l}1.81 \\
1.86\end{array}$ \\
\hline
\end{tabular}

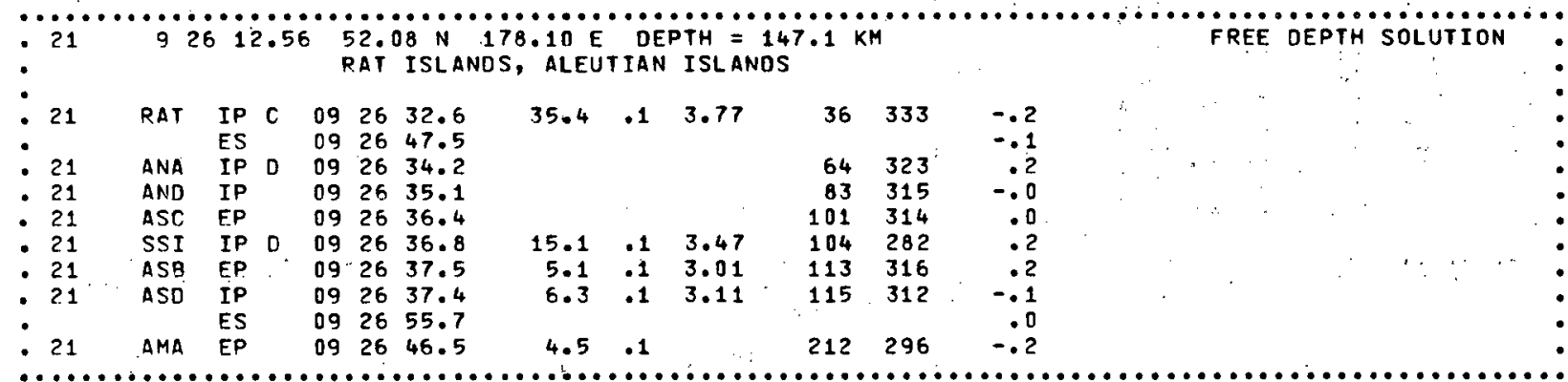

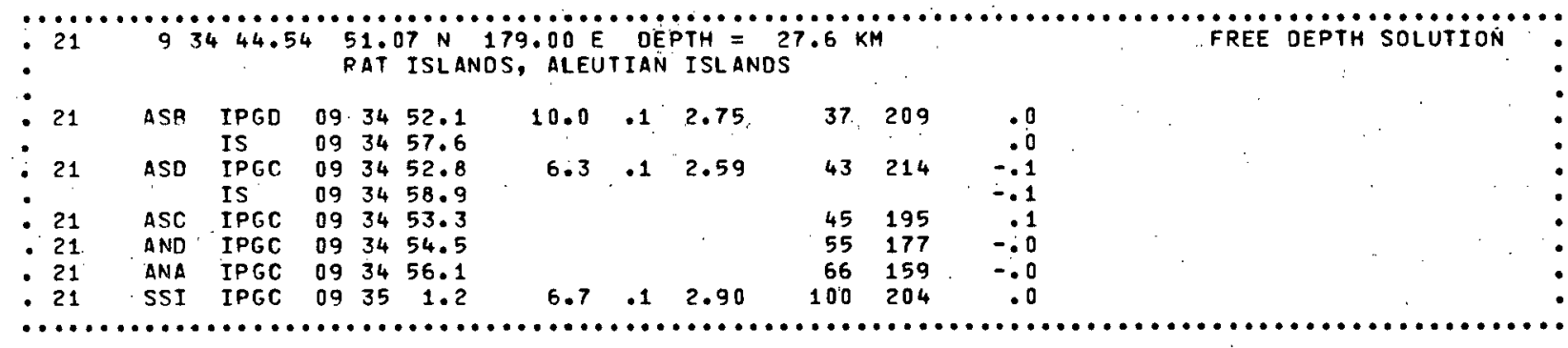

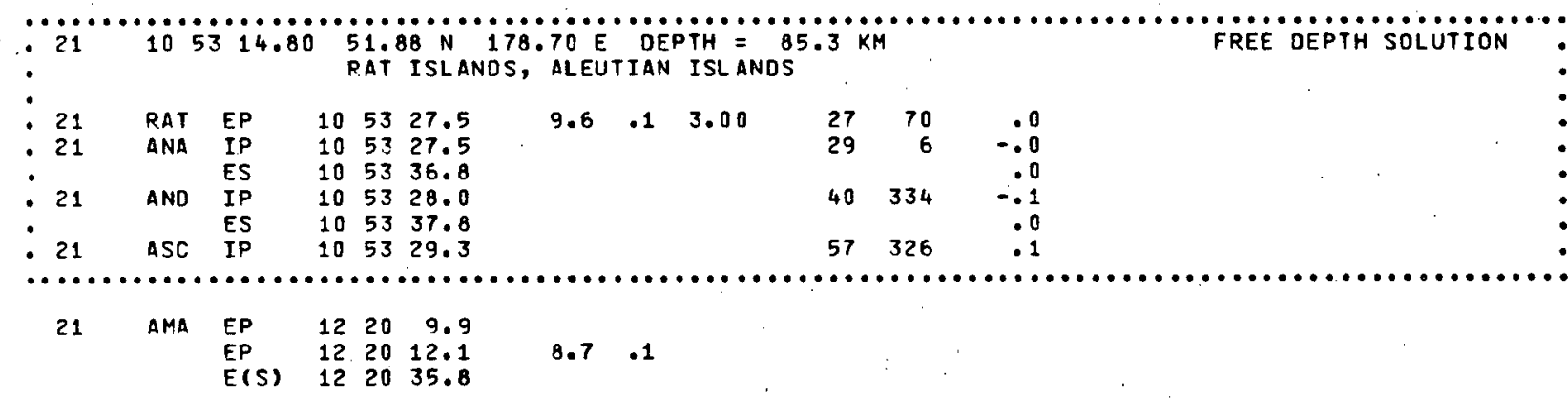

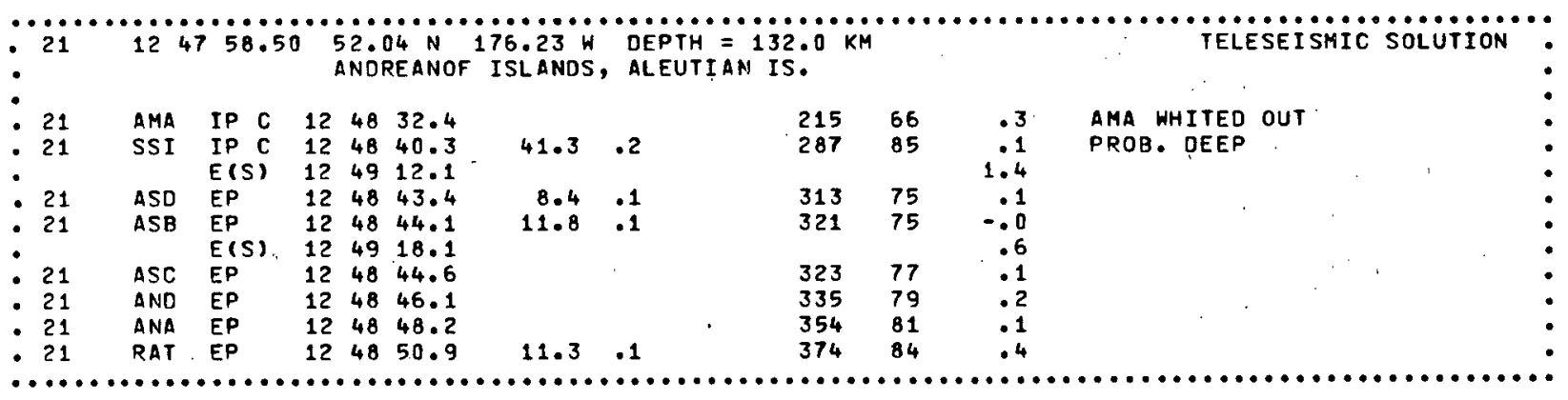




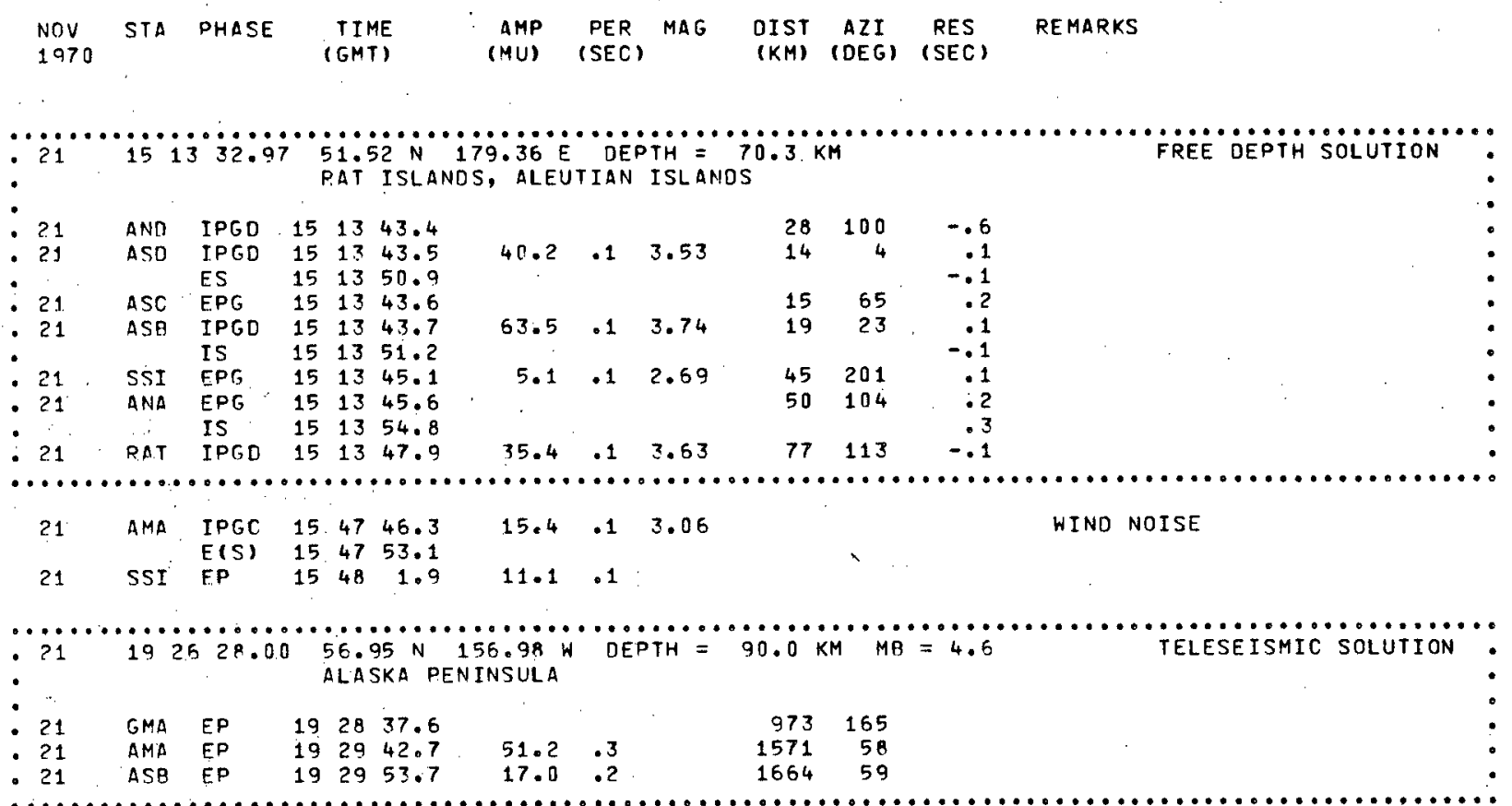

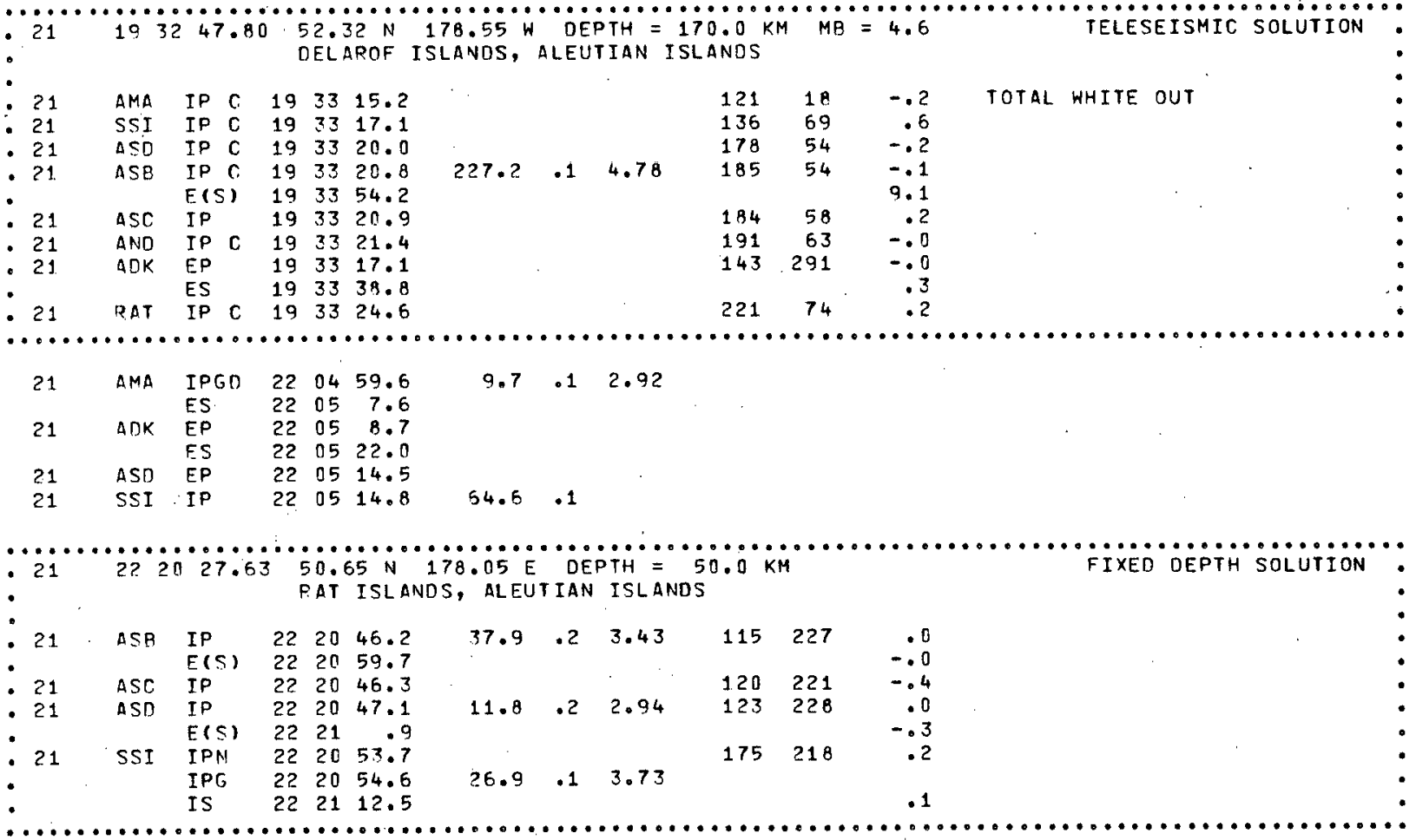




NOV STA PHASE TIME
1970

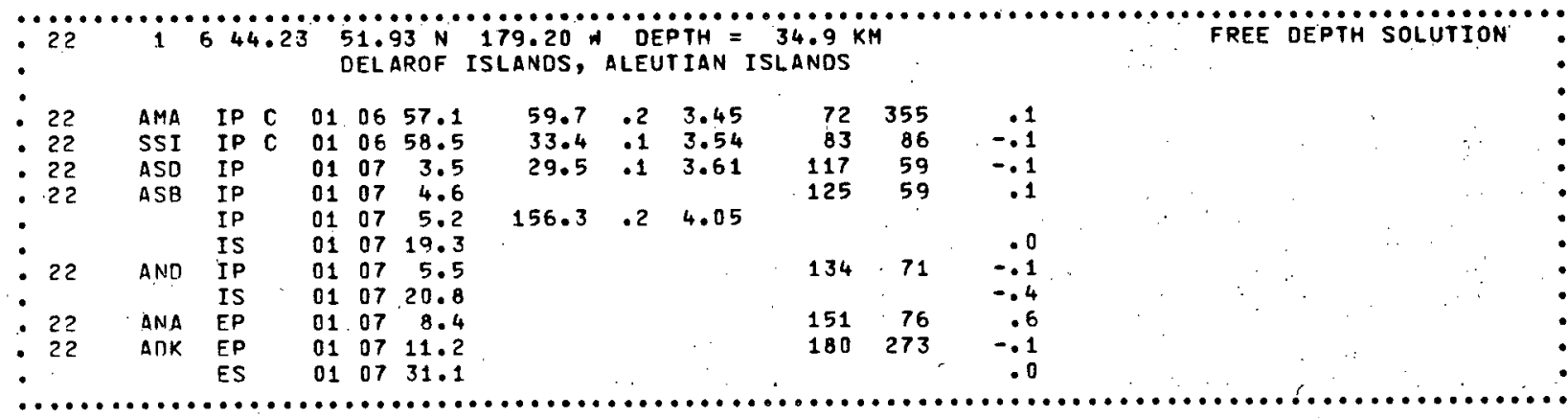

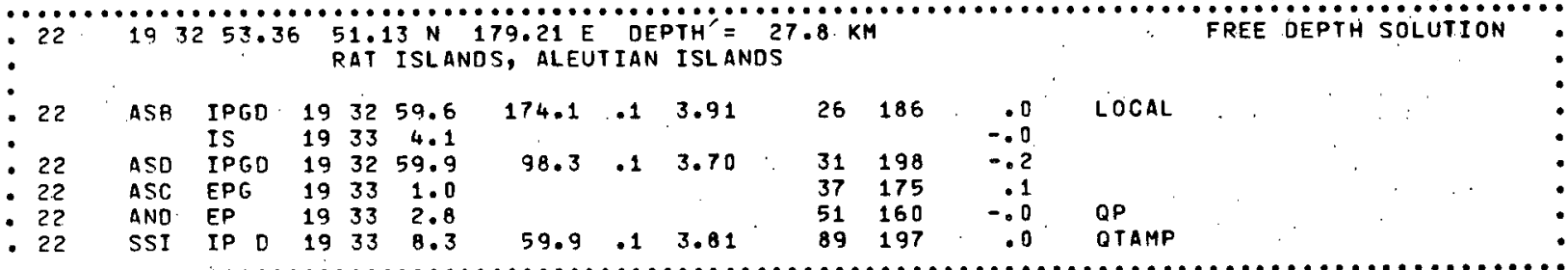

............................................................................

23
1

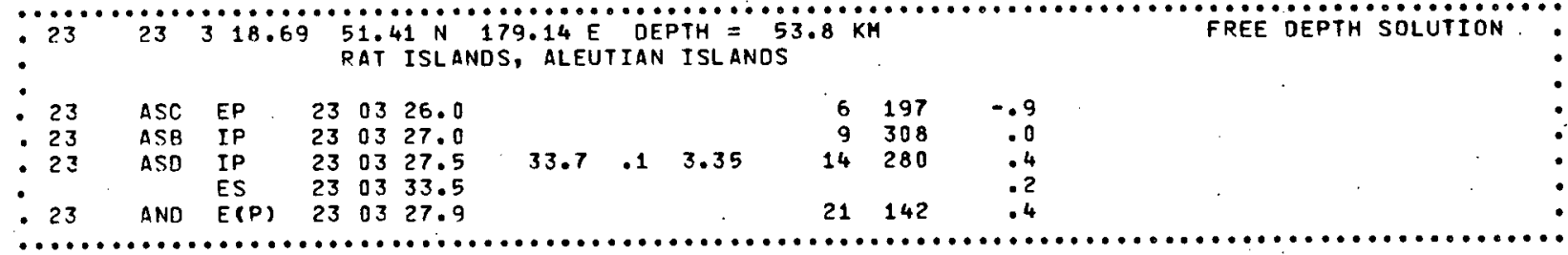

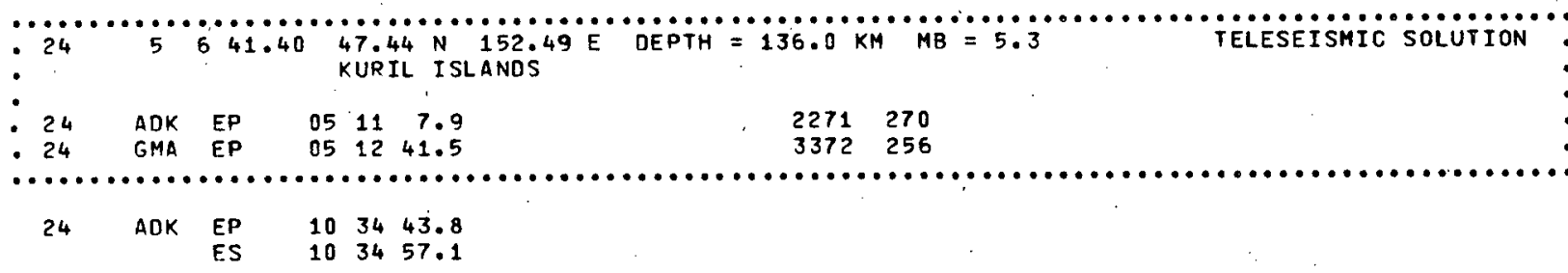

\begin{tabular}{|c|c|c|c|c|c|c|c|c|c|c|c|c|}
\hline $\begin{array}{l}24 \\
24 \\
24 \\
24\end{array}$ & $\begin{array}{l}A S B \\
\text { ASD } \\
\text { AND } \\
\text { SSI }\end{array}$ & $\begin{array}{l}\text { IPGD } \\
\text { E } S \text { S } \\
\text { IPGD } \\
\text { IPGD } \\
\text { EP }\end{array}$ & $\begin{array}{l}11 \\
11 \\
11 \\
11 \\
11\end{array}$ & $\begin{array}{l}29 \\
29 \\
29 \\
29 \\
29\end{array}$ & $\begin{array}{r}4.5 \\
8.8 \\
5.2 \\
6.8 \\
13.5\end{array}$ & 473.4 & .2 & 4.02 & $\begin{array}{l}26 \\
33 \\
44 \\
89\end{array}$ & $\begin{array}{l}217 \\
223 \\
174 \\
206\end{array}$ & $\begin{array}{r}.0 \\
.0 \\
-.2 \\
.0 \\
.0\end{array}$ & $\begin{array}{l}\text { QS,LOCAL } \\
\text { QP, OBSCURED }\end{array}$ \\
\hline
\end{tabular}




\begin{tabular}{|c|c|c|c|c|c|c|c|c|c|c|c|c|}
\hline $\begin{array}{l}\text { Nov } \\
1970\end{array}$ & STA & PHASE & & $\begin{array}{l}\text { TIM } \\
\text { I GMT }\end{array}$ & & $\begin{array}{l}\text { AMP } \\
(M U)\end{array}$ & $\begin{array}{c}\text { PER } \\
(S E C)\end{array}$ & MAG & $\begin{array}{l}\text { OIST } \\
\text { (KM) }\end{array}$ & $\begin{array}{c}A Z I \\
\text { (DEG) }\end{array}$ & $\begin{array}{c}\text { RES } \\
\text { (SEC) }\end{array}$ & REMARKS \\
\hline 24 & AMA & IPGD & 19 & $\begin{array}{l}58 \\
58\end{array}$ & $\begin{array}{r}.3 \\
8.8\end{array}$ & & & & & & & AMA WHITE OUT \\
\hline 24. & $A D K$ & & $\begin{array}{l}19 \\
19\end{array}$ & $\begin{array}{l}58 \\
58\end{array}$ & $\begin{array}{l}10.7 \\
26.1\end{array}$ & & & & & & & \\
\hline 24 & SSI & $\begin{array}{l}\text { EP } \\
\text { IS }\end{array}$ & $\begin{array}{l}19 \\
19\end{array}$ & $\begin{array}{l}58 \\
58\end{array}$ & $\begin{array}{l}15.9 \\
36.0\end{array}$ & 21.4 & .1 & 3.63 & & & & OTAMP \\
\hline 25 & $40 K$ & $\begin{array}{l}\text { EP D } \\
\text { ES }\end{array}$ & $\begin{array}{l}02 \\
02\end{array}$ & $\begin{array}{l}34 \\
34\end{array}$ & $\begin{array}{l}12.9 \\
25.1\end{array}$ & & & & & & & \\
\hline
\end{tabular}

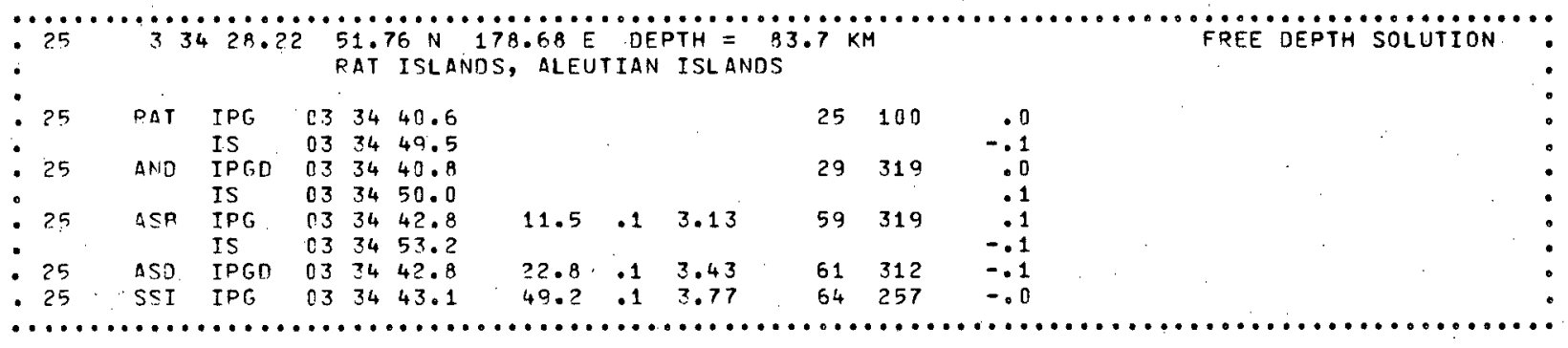

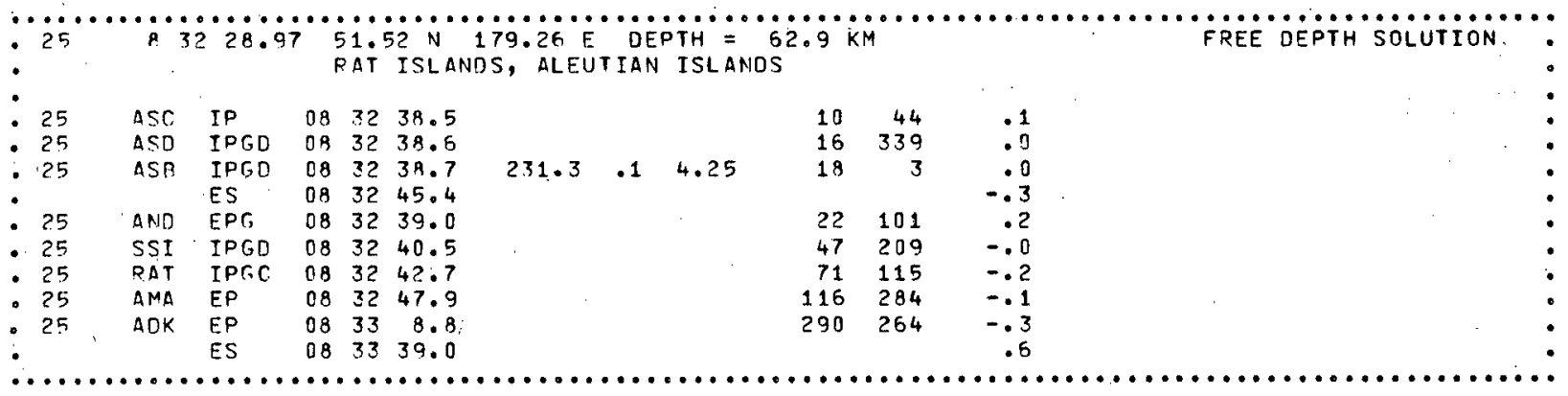

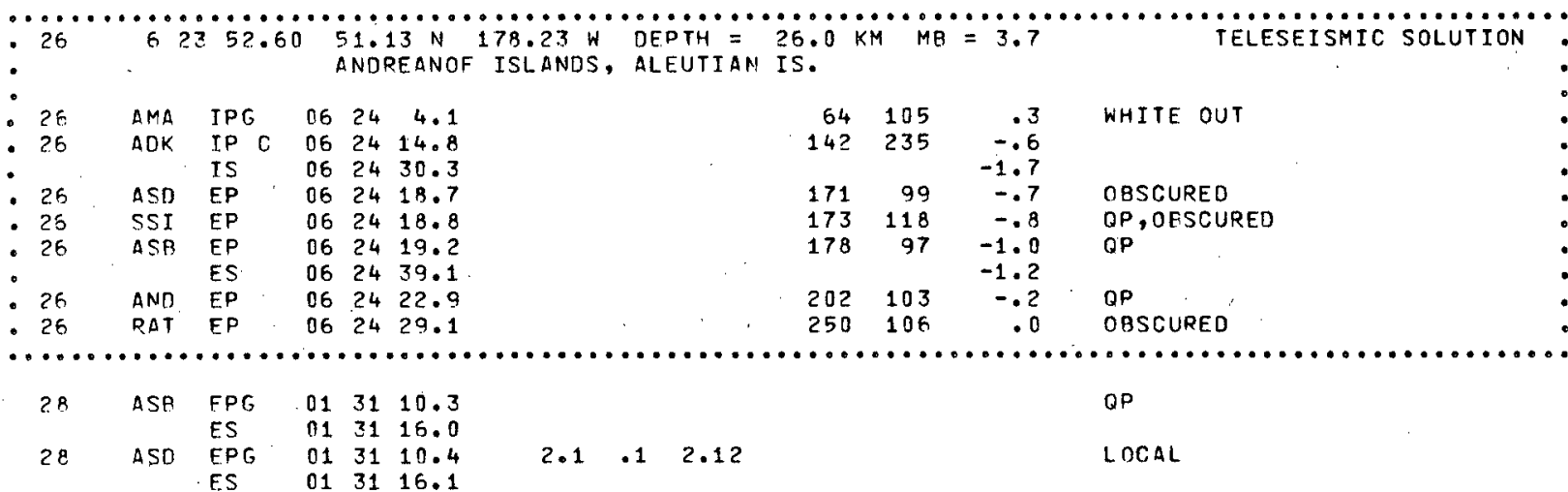

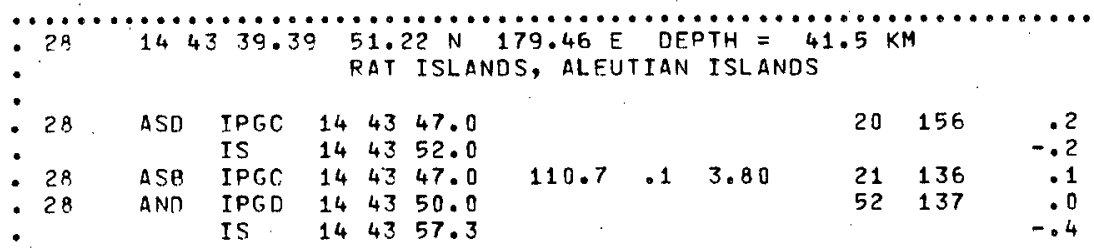




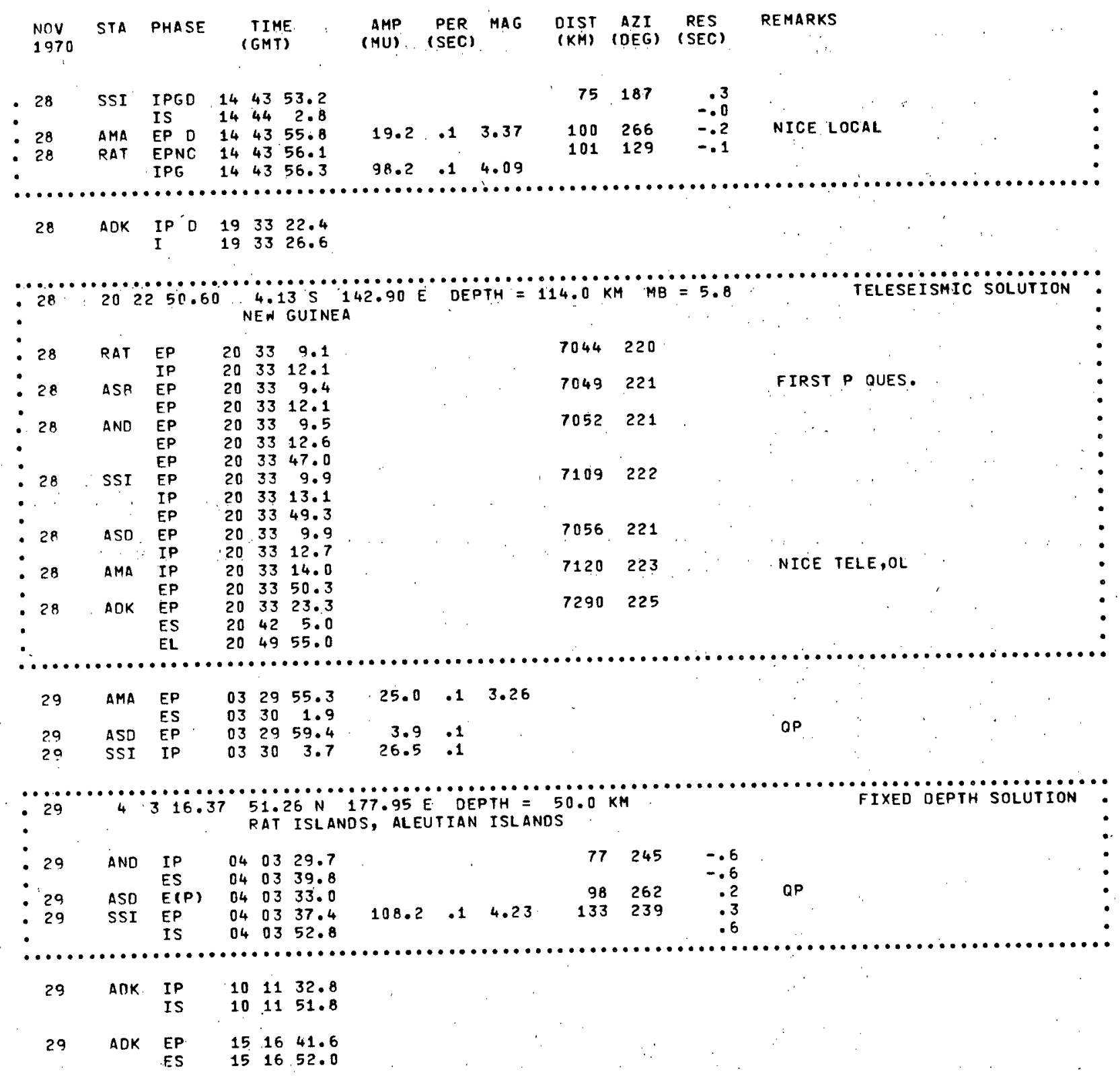

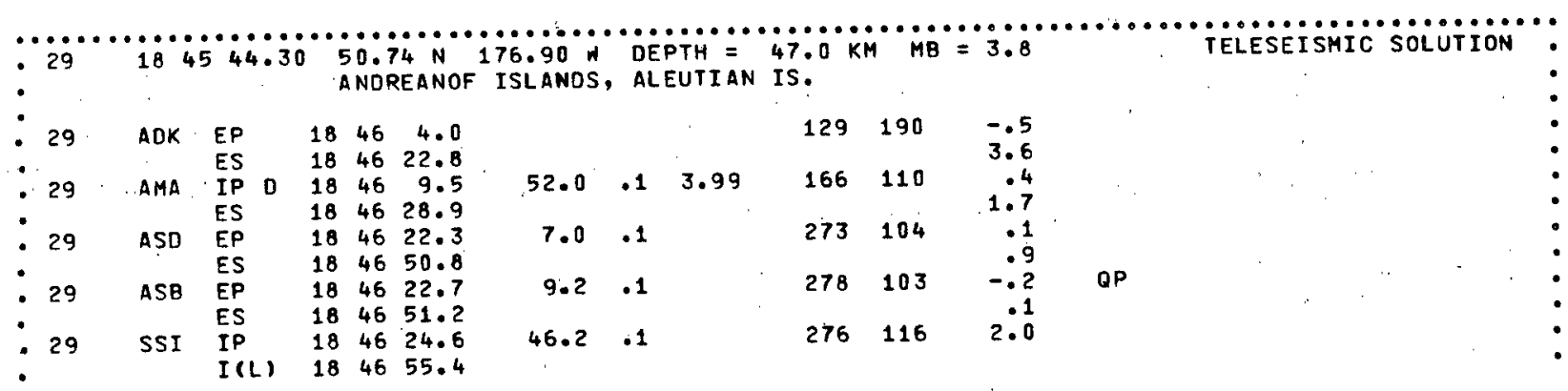




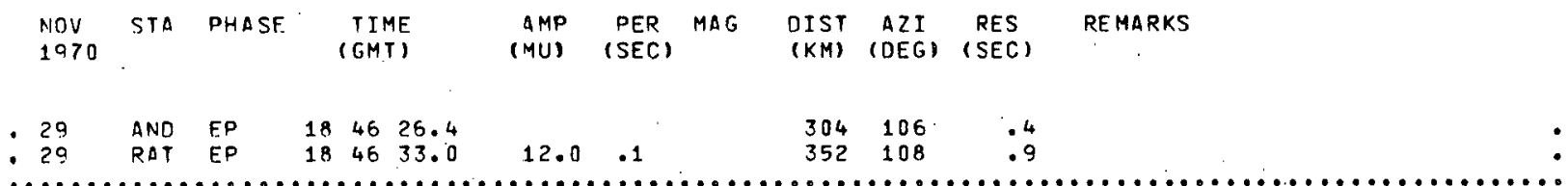

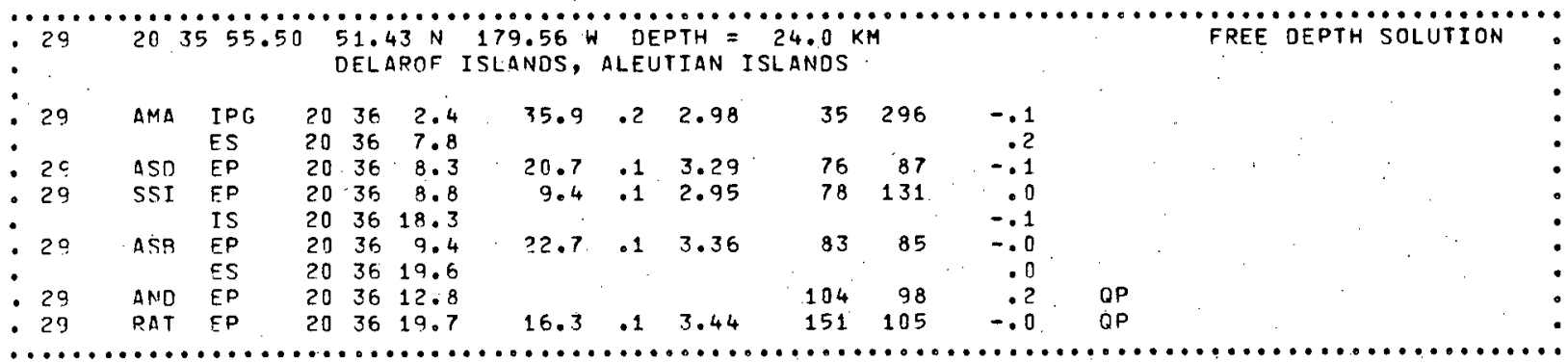

$$
29 \quad A D K \text { EP } 214459.1
$$

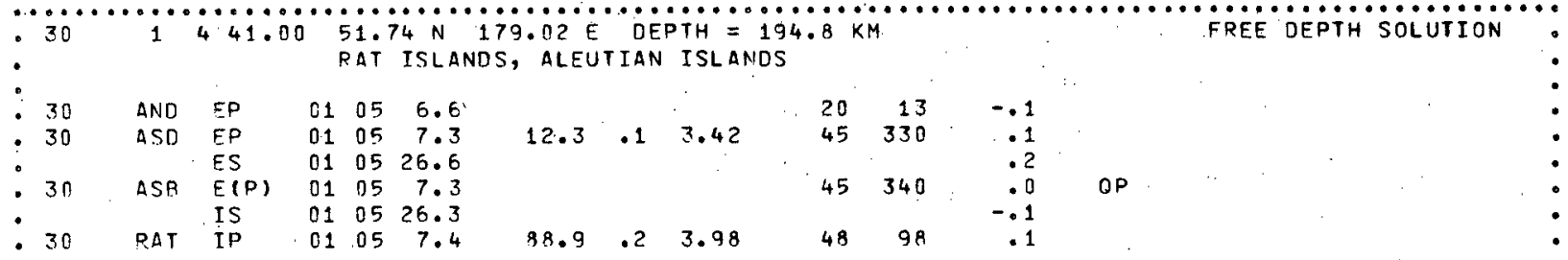

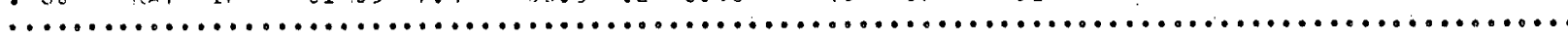

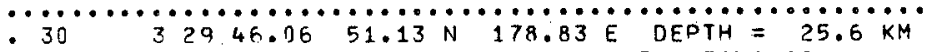

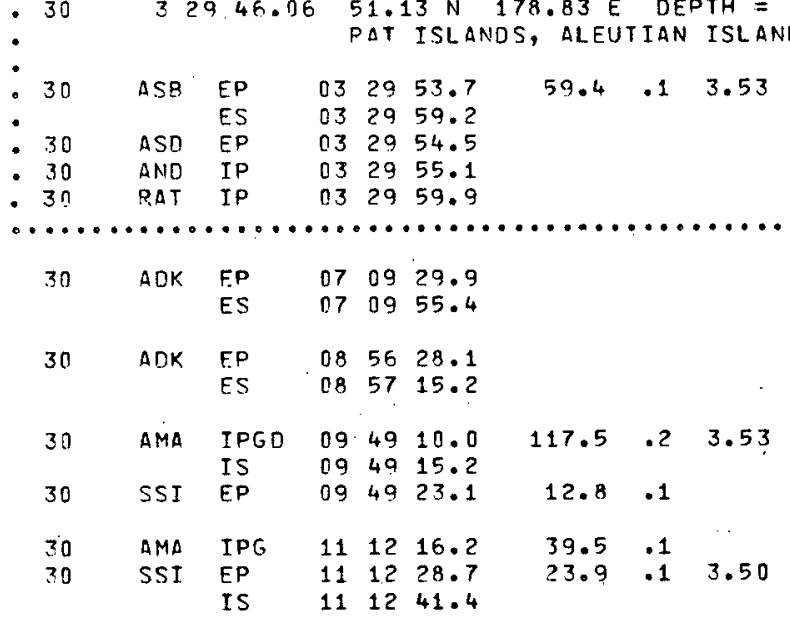

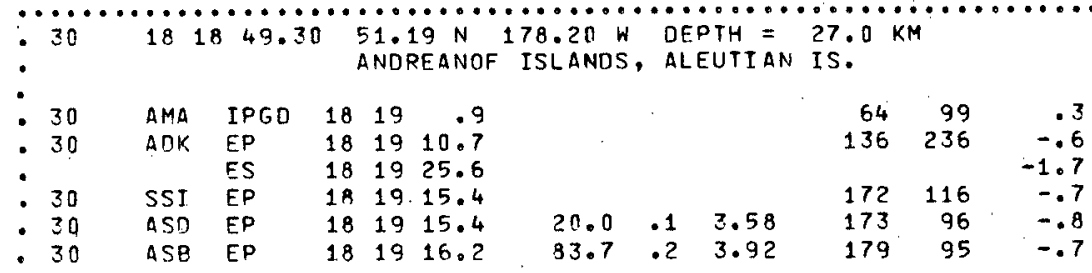




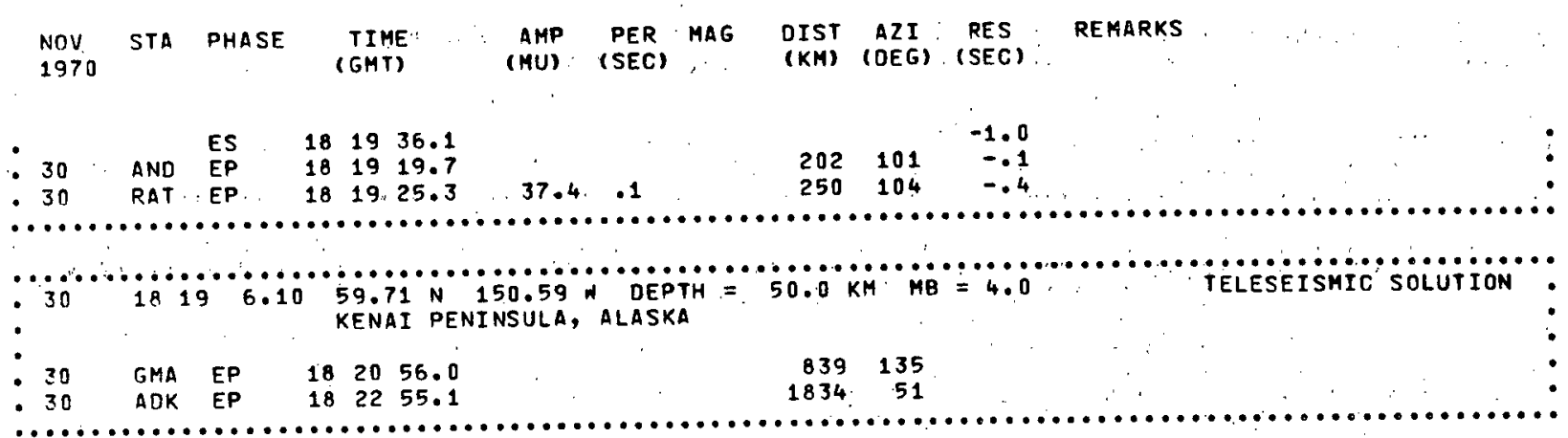




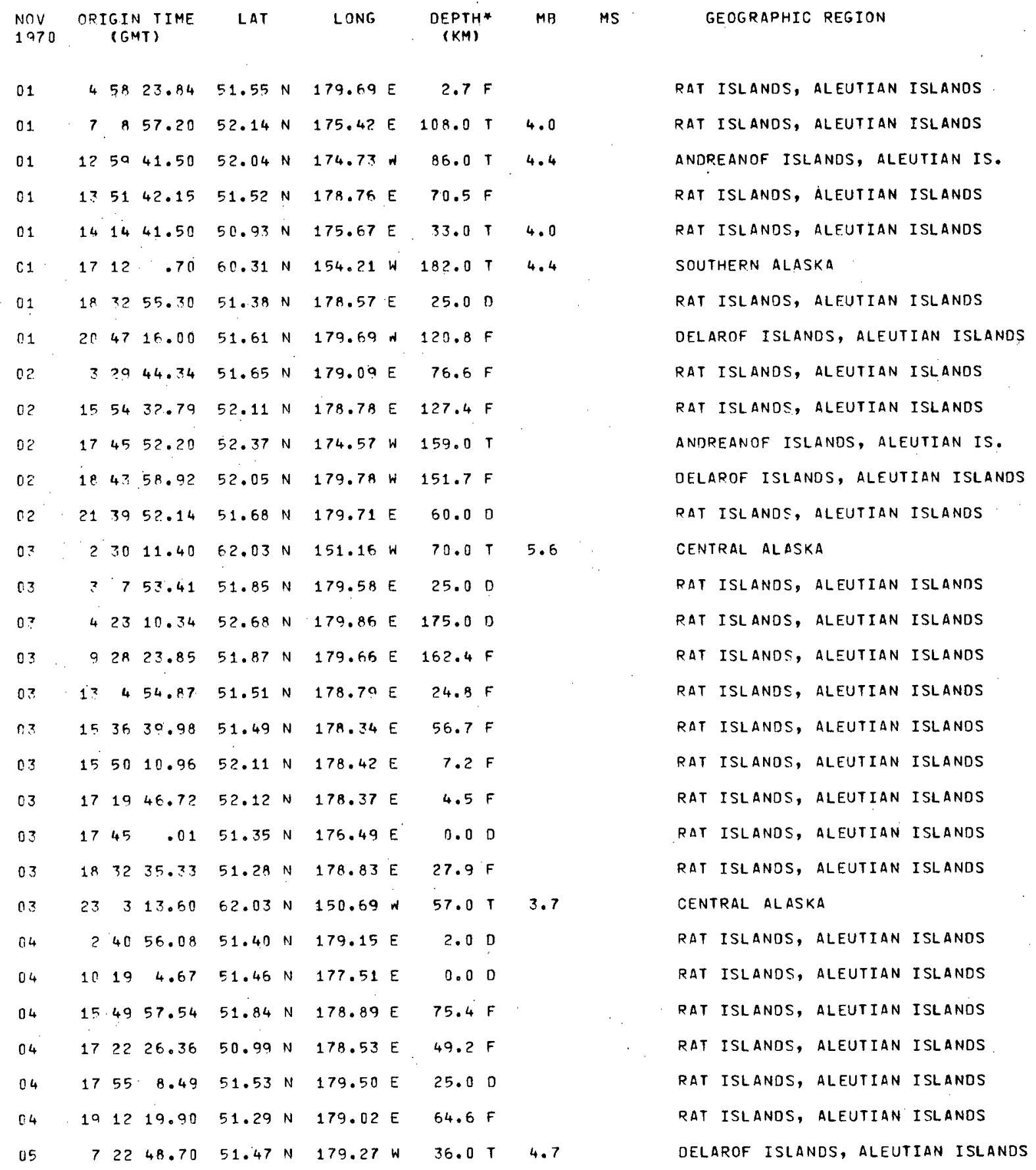




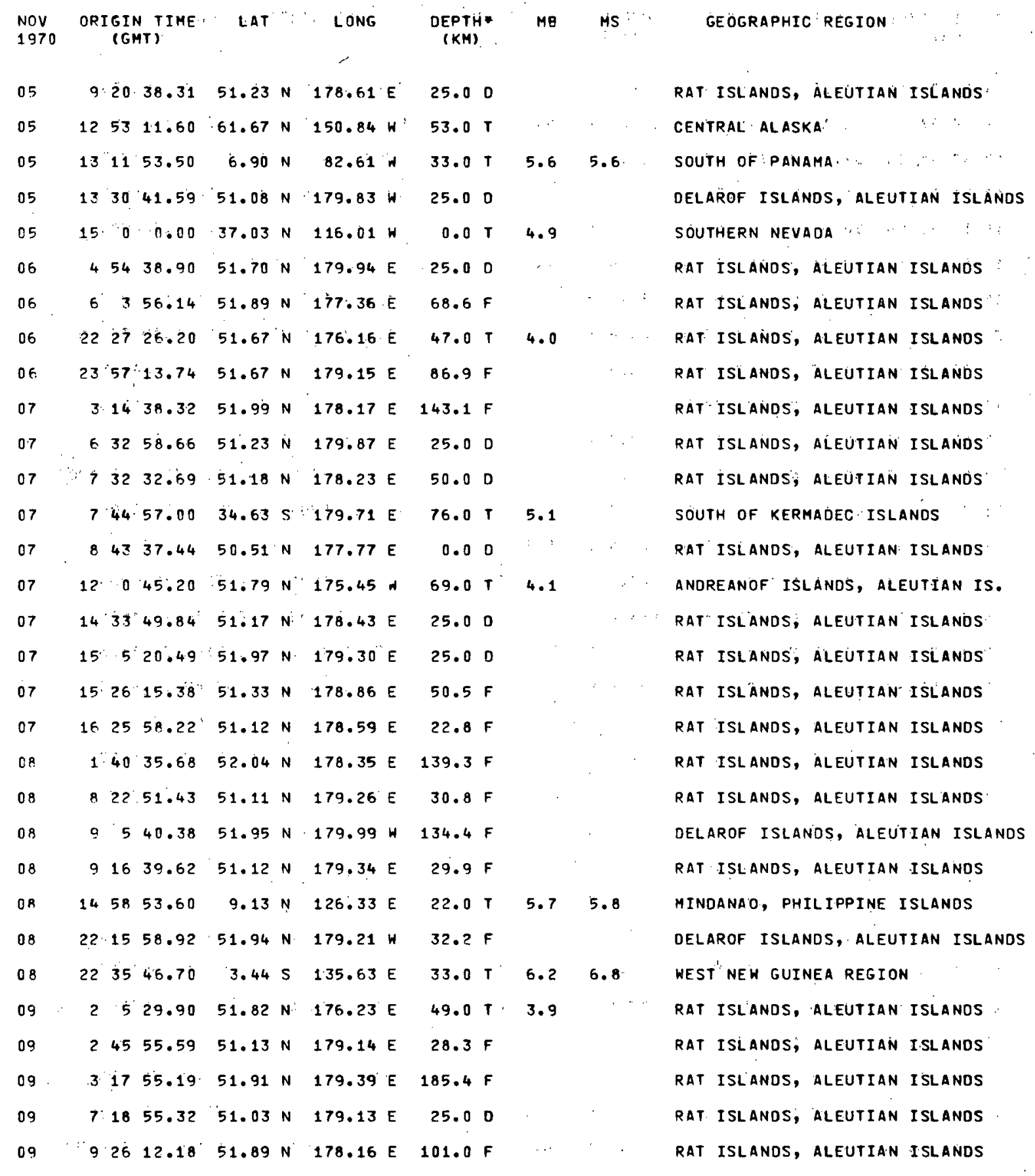




\begin{tabular}{|c|c|c|c|c|c|c|c|c|c|c|c|c|}
\hline $\begin{array}{l}\text { NOV } \\
1970\end{array}$ & $O P$ & & $\begin{array}{l}N \text { T IME } \\
M T \text { ) }\end{array}$ & LAT & & LONG & & $\begin{array}{r}\text { DEPTH } \\
\text { (KM) }\end{array}$ & & $M B$ & & MS \\
\hline 09 & 12 & 17 & $33 \cdot 42$ & 51.50 & $N$ & 179.00 & E & 66.9 & $F$ & & & \\
\hline 09 & 12 & 25 & 32.05 & 51.58 & $N$ & 178.82 & $E$ & 75.2 & $F$ & - & & \\
\hline 39 & 12 & 45 & 25.23 & 51.13 & $N$ & 178.58 & $E$ & 0.0 & $D$ & & & \\
\hline 09 & 16 & 46 & 29.53 & 50.81 & $N$ & 179.56 & $\downarrow$ & 25.0. & 0 & & & \\
\hline 09 & 20 & 42 & 22.06 & 51.67 & $N$ & 179.08 & $\mathbf{W}$ & 91.4 & $F$ & & & \\
\hline 09 & 21 & 28 & 45.46 & 52.25 & $N$ & 178.45 & $\omega$ & 186.5 & $F$ & & & \\
\hline 09 & $2 ?$ & 35 & $11 \cdot 12$ & $51 \cdot 21$ & $N$ & 178.77 & $E$ & 28.4 & $F$ & & & \\
\hline 10 & 0 & 26 & 21.60 & 34.60 & $\mathrm{~N}$ & 136.71 & $E$. & 349.0 & $T$ & 5.3 & & . \\
\hline 10 & 2 & 23 & 44.50 & 52.38 & $\mathrm{~N}$ & 179.87 & $E$ & 160.0 & $F$ & & & \\
\hline 10 & 5 & 30 & 1.51 & $51 \cdot 43$ & $N$ & 178.83 & $\mathrm{E}$ & 42.6 & $F$. & $\cdot$ & & \\
\hline 10 & 5 & 49 & 56.53 & 51.58 & $\mathrm{~N}$ & 179.67 & W & 189.9 & $F$ & & & \\
\hline 10 & 13 & 12 & 56.08 & 51.70 & $N$ & 179.81 & W & 25.0 & 0 & & & \\
\hline 10 & 15 & 57 & 41.83 & 50.58 & $N$ & 178.19 & $E$ & 50.0 & $D$ & . & & . \\
\hline 10 & 16 & $?$ & 17.67 & 50.87 & $N$ & 178.30 & $E$ & 25.0 & 0 & . & & \\
\hline 10 & 17 & 39 & 10.32 & 50.82 & $\mathrm{~N}$ & 178.22 & $E$ & 25.0 & D & $\therefore$ & $\therefore$ & \\
\hline 10 & 20 & 3 & 3.64 & 51.58 & $\mathrm{~N}$ & 179.39 & $E$ & 18.6 & $F$ & . & $\cdot:$ & \\
\hline 11 & 0 & 53 & 5.59 & 51.71 & $N$ & 179.94 & E & 16.1 & $\mathbf{F}$ & $\because$ & & \\
\hline 11 & ? & 26 & 48.36 & 51.69 & $N$ & 178.61 & $H$ & 144.1 & $\mathrm{~F}$ & & & \\
\hline 11 & 14 & 52 & 42.69 & 51.40 & $N$ & 178.78 & $E$ & 44.9 & $\mathbf{F}$ & & & \\
\hline 11 & 17 & 37 & 39.85 & $52 \cdot 13$ & $N$ & 179.24 & $n$ & 50.0 & ח & & & \\
\hline 11 & 20 & 23 & 15.78 & 51.92 & $N$ & 179.24 & $E$ & 130.9 & $F$ & & & \\
\hline 11 & 21 & 1 & $54 \cdot 34$ & 51.50 & $\mathrm{~N}$ & 178.69 & $E$ & $21 \cdot 3$ & $F$ & & & \\
\hline 12 & 1 & 56 & 52.85 & 51.70 & $\mathrm{~N}$ & 178.23 & $E$ & 82.6 & $F$ & & & \\
\hline 12 & 5 & 5 म & 46.19 & 51.18 & $N$ & 179.53 & W & 50.0 & D & & & \\
\hline 12 & 6 & 9 & 14.79 & 51.51 & $N$ & 179.96 & $E$ & 81.3 & $\mathrm{~F}$ & & & \\
\hline 12 . & $\epsilon$ & 7 & 12.40 & 5.05 & $\mathrm{~s}$ & 145.06 & $\varepsilon$ & 15.0 & $T$ & 5.8 & & 6.5 \\
\hline 12 & 7 & 22 & 33.00 & 51.70 & $\mathrm{~N}$ & 178.94 & $\omega$ & 100.0 & 0 & & & \\
\hline 12 & 9 & 31 & 56.86 & 51.54 & $\mathrm{~N}$ & 179.14 & $E$ & 20.4 & $F$ & . & & \\
\hline $1 ?$ & 16 & 16 & 24.40 & 62.45 & $N$ & $150 \cdot 10$ & $w$ & 48.0 & $T$ & & & \\
\hline 12 & 17 & 45 & 40.50 & 52.19 & $N$ & 173.74 & $E$ & 136.0 & $T$ & 3.9 & & \\
\hline 13 & 4 & 22 & 57.39 & 50.39 & N & 177.71 & $E$ & 15.7 & $F$ & & & \\
\hline
\end{tabular}

RAT ISLANDS, ALEUTIAN ISLANDS

RAT ISLANOS, ALEUTIAN ISLANOS

RAT ISLANDS, ALEUTIAN ISLANOS

DELAROF ISLANDS, ALEUTIAN ISLANDS

DELAROF ISLANDS, ALEUTIAN ISLANDS

DELAROF ISLANDS, ALEUTIAN ISLANOS

RAT ISLANDS, ALEUTIAN ISLANDS.

SOUTHERN HONSHU, JAPAN

RAT ISLANDS, ALEUTIAN ISLANOS

RAT ISLANDS, ALEUTIAN ISLANDS

DELAROF ISLANDS, ALEUTIAN ISLANDS

DELAROF ISLANOS, ALEUTIAN ISLANOS

RAT ISLANDS, ALEUTIAN ISLANOS

RAT ISLANOS, ALEUTIAN ISLANDS

RAT ISLANDS, ALEUTIAN ISLANDS

RAT ISLANOS, ALEUTIAN ISLANDS

RAT ISLANOS, ALEUTIAN ISLANDS

DELAROF ISLANDS, ALEUTIAN ISLANDS

RAT ISLANDS, ALEUTIAN ISLANDS

DELAROF ISLANDS, ALEUTIAN ISLANDS

RAT ISLANDS, ALEUTIIAN ISLANDS

RAT ISLANDS, ALEUTIAN ISLANDS

RAT ISLANDS, ALEUTIAN ISLANOS

DELAROF ISLANDS, ALEUTIAN ISLANOS

RAT ISLANOS, ALEUTIAN ISLANDS

EAST NEW GUINEA REGION

DELAROF ISLANDS, ALEUTIAN ISLANDS

RAT ISLANDS, ALEUTIAN ISLANDS

CENTRAL ALASKA

NEAR ISLANDS, ALEUTIAN ISLANDS

RAT ISLANOS, ALEUTIAN ISLANDS 


\begin{tabular}{|c|c|c|c|c|c|c|c|c|c|c|c|c|}
\hline $\begin{array}{l}\text { Nov } \\
1970\end{array}$ & ORI & $\begin{array}{l}\text { I GIN } \\
\text { IGM }\end{array}$ & $\begin{array}{l}\text { NTT TIME } \\
\text { MT) }\end{array}$ & LAT & & LONG & & $\begin{array}{l}\text { DEPTH } \\
\text { (KM) }\end{array}$ & & MB & ins & GEOGRAPHIC REGION \\
\hline 13 & 14 & 16 & 18.00 & 11.95 & N & $123: 97$ & $\mathbf{E}$ & $15.0 T$ & $\boldsymbol{T}$ & 5.4 & 6.3 & LEYTE, PHILIPPINE ISLANOS \\
\hline 13 & 13 & 10 & 25.40 & 51.62 & N & 175.34 & $\omega$ & 51.0 & T & 4.9 & & ANOREANOF ISLANDS, ALEUTIAN IS. \\
\hline 13 & 16 & 3 & 16.80 & 52.291 & N & 173.97 & W & 132.0 & $T$ & 3.7 & & ANDREANOF ISLANOS, ALEUTIAN IS. \\
\hline 13 & 16 & 41 & 42.30 & 51.201 & $N$ & 178.78 & $E$ & 25.0 & D & & & RAT ISLANDS, ALEUTIAN ISLANOS \\
\hline 13 & 19. & 2 & 19.20 & 51.80 & $N$ & 176.79 & $E$ & 66.0 & $T$. & 4.5 & & RAT ISLANOS, ALEUTIAN ISLANDS \\
\hline 13 & 20 & 57 & 55.49 & 51.93 & $N$ & 178.29 & $E$ & 138.7 & $\mathbf{F}$ & & $\because$ & RAT ISLANOS, ALEUTIAN ISLANDS \\
\hline 14 & 4. & 51 & 37.80 & 12.64 & $N$ & 143.30 & E & 95.0 & $\mathbf{T}$ & 5.5 & & SOUTH OF MARIANA ISLANDS \\
\hline 14 & 7 & 58 & 19.80 & 22.71 & $\mathrm{~N}$ & 121.34 & E & 28.0 & $T$ & 5.7 & 6.1 & TAIHAN REGION \\
\hline 14 & 18 & 53 & 30.60 & 58.76 & $\mathrm{~N}$ & 149.73 & $\omega$ & 33.0 & $\top$ & 3.9 & . & GULF OF ALASKA \\
\hline 14 & 21 & 10 & 7.86 & 51.63 & $\mathrm{~N}$ & 177.97 & $E$ & 56.7 & $\boldsymbol{F}^{\prime}$ & & & RAT ISLANDS, ALEUTIAN ISLANDS \\
\hline $14^{\circ}$ & 23 & 1 & 25.15 & 50.88 & $\mathrm{~N}$ & 177.53 & E & 25.0 & 0 & & & RAT ISLANOS, ALEUTIAN ISLANOS \\
\hline 14 & 23 & 46 & 52.10 & 51.59 & $\mathrm{~N}$ & 179.39 & $\mathbf{E}$ & 82.0 & $T$ & 4.1 & & RAT ISLANDS, ALEUTIAN ISLANDS \\
\hline 15 & $B$ & 3 & 29.18 & 51.18 & $\mathrm{~N}$ & 178.69 & $E$ & 25.4 & $\mathbf{F}$ & & & RAT ISLANDS, ALEUTIAN ISLANDS \\
\hline 15 & 9 & 48 & 23.15 & 51.28 & $\mathrm{~N}$ & 179.62 & E & 54.7 & $\mathbf{F}$ & & & RAT ISLANDS, ALEUTIAN ISLANDS \\
\hline 15 & 12 & 5 & 44.64 & 51.66 & $\mathrm{~N}$ & 177.81 & $E$ & 59.8 & $\mathbf{F}$ & & 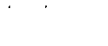 & RAT ISLANDS, ALEUTIAN ISLANDS \\
\hline 15 & 15 & 57 & 36.56 & 51.59 & $\mathrm{~N}$ & 177.74 & $\mathrm{E}$ & 56.3 & $\mathbf{F}$ & & & RAT ISLANDS, ALEUTIAN ISLANDS \\
\hline 15 & 17 & 1 & 21.98 & 50.12 & N & 179.31 & $w$ & 25.0 & 0 & & & DELAROF ISLANDS, ALEUTIAN ISLANDS \\
\hline 15 & 22 & 43 & 48.63 & $51: 10$ & $\mathbf{N}$ & 179.98 & $\omega$ & 35.5 & $F$ & & & DELAROF ISLANDS, ALEUTIAN ISLANDS \\
\hline 16 & 2 & 54 & 49.64 & 52.25 & $\mathrm{~N}$ & 179.97 & E & 230.2 & $F$ & 3.7 & & RAT ISLANDS, ALEUTIAN ISLANDS \\
\hline 16 & 6 & 44 & 21.40 & 6.08 & $S$ & 148.58 & $E$ & 81.0 & $T$ & 5.5 & & NEW BRITAIN REGION \\
\hline 16 & 14 & 5 & 5.90 & 50.89 & $\mathrm{~N}$ & 179.83 & $\varepsilon$ & $32 \cdot 0$ & $\top$ & 4.1 & r. & RAT ISLANDS, ALEUTIAN ISLANOS \\
\hline 16 & $1 \mathrm{~A}$ & 4 & 43.10 & 57.84 & $\mathbf{N}$ & 154.32 & H & 33.0 & 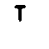 & & & KODIAK ISLAND REGION. \\
\hline 17 & 13 & 13 & 4.68 & 51.40 & $N$ & $9 \cdot 91$ & E & 66.4 & $\mathbf{F}$ & & 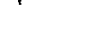 & RAT ISLANDS, ALEUTIAN ISLANDS \\
\hline 17 & 14 & 21 & 42.10 & 51.41 & $\dot{N}$ & $178 \cdot 77$ & E & 45.0 & $\mathbf{F}$ & & & RAT ISLANDS, ALEUTIAN ISLANDS \\
\hline 17 & 16 & 6 & 31.71 & 51.10 & $\mathrm{~N}$ & 179.47 & $w$ & 0.0 & 0 & & & DELAROF ISLANDS, ALEUTIAN ISLANDS \\
\hline 17 & 22 & 42 & 58.93 & 51.44 & $\mathrm{~N}$ & 178.25 & H & $24 \cdot 0$ & $\mathbf{F}$ & & & ANOREANOF ISLANOS, ALEUTIAN IS. \\
\hline 18 & 1 & 57 & 25.90 & 6.08 & $\mathbf{S}$ & 154.43 & E & 15.0 & $\mathbf{T}$ & 5.2 & 5.6 & SOLOHON ISLANDS \\
\hline 18 & 3 & 18 & $37 \cdot 29$ & 51.12 & $\mathrm{~N}$ & 179.93 & 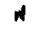 & 26.5 & $\mathbf{F}$ & & & DELAROF ISLANDS, ALEUTIAN ISLANDS \\
\hline 18 & 8 & 45 & 20.28 & 51.91 & N & 79.27 & $\mathbf{H}$ & 25.2 & $\mathbf{F}$ & & & DELAROF ISLANDS, ALEUTIAN ISLANDS \\
\hline 18 & & 45 & 28.50 & 3.66 & $\mathrm{~s}$ & 148.91 & $E$ & 33.0 & $\top$ & 4.9 & 5.8 & BISMARCK SEA \\
\hline 18 & 10 & 41 & 3.44 & 51.15 & N & $178 \cdot 27$ & $E$ & 50.0 & D & & & RAT ISLANDS, ALEUTIAN ISLANDS \\
\hline
\end{tabular}




\begin{tabular}{|c|c|c|c|c|c|c|c|c|c|c|c|}
\hline $\begin{array}{l}\ln V \\
1.970\end{array}$ & OPI & $\begin{array}{l}G I N \\
\text { IGM }\end{array}$ & $\begin{array}{l}\text { TIME } \\
\text { (T) }\end{array}$ & LAT & & LONG & & $\begin{array}{l}\text { DEPTH* } \\
\text { (KM) }\end{array}$ & & $M B$ & MS \\
\hline 18 & 11 & 52 & 38.68 & 50.34 & $N$ & 178.31 & $E$ & 0.00 & 0 & & \\
\hline If. & 12 & 11 & 18.31 & 51.47 & $N$ & 179.45 & $E$ & 50.00 & D & & \\
\hline 18 & $1 ?$ & 23 & $18 . \cap n$ & 35.15 & $N$ & 35.74 & $W$ & 33.0 & $\mathbf{T}$ & 5.4 & 6.0 \\
\hline 18 & $1 \mathrm{f}$ & 47 & 32.26 & 51.13 & $N$ & 179.20 & $E$ & $25.6 \mathrm{~F}$ & $F$ & & \\
\hline 18 & 16 & 43 & 14.10 & 21.89 & $S$ & 175.17 & $E$ & $570.0 \mathrm{~T}$ & $\uparrow$ & 5.5 & \\
\hline 18 & ?? & 8 & 38.99 & 51.69 & $N$ & 178.70 & $E$ & 83.0 & $F$ & & \\
\hline 18 & $2 ?$ & 52 & 32.32 & 51.28 & N & 179.90 & $E$ & 25.0 & 0 & & \\
\hline 12 & $2 ?$ & 16 & 33.99 & $51 \cdot 19$ & $N$ & 179.44 & $E$ & $38.8 \mathrm{~F}$ & $F$ & & \\
\hline 19 & 0 & 30 & 12.47 & 51.12 & $N$ & 179.43 & $W$ & 0.00 & D & & \\
\hline 19 & 0 & 31 & $5 F .54$ & 50.99 & $N$ & 179.84 & $w$ & $29.6 \mathrm{~F}$ & $F$ & & \\
\hline 19 & h & 58 & 9.20 & 50.17 & $N$ & 179.13 & $E$ & 50.0 & D & & \\
\hline 19 & 8 & 4 & 58.27 & 51.82 & $N$ & 179.99 & $\omega$ & $102.4 \mathrm{~F}$ & $F$ & & \\
\hline $1^{a}$ & 11 & 20 & 38.50 & 51.89 & $N$ & 179.32 & $W$ & $106.7 \mathrm{~F}$ & $F$ & & \\
\hline 19 & 14 & $3 n$ & 37.55 & 52.14 & $N$ & 179.88 & $w$ & $178.4 F$ & $F$ & & \\
\hline 19 & 16 & 29 & 58.49 & $51 \cdot 23$ & $N$ & 179.81 & $E$ & 2.5 .0 & 0 & & \\
\hline 19 & 17 & $2 n$ & $3 R \cdot A 5$ & 51.08 & $N$ & 178.44 & $E$ & 25.0 & D & & \\
\hline 19 & 21 & 5 & 47.55 & 51.20 & $N$ & 178.51 & $E$ & 24.8 & $\mathrm{~F}$ & & \\
\hline 19 & 22 & $51^{\circ}$ & 48.28 & $51 \cdot 31$ & $N$ & 179.85 & $w$ & 24.7 & $\mathrm{~F}$ & & \\
\hline 20 & 2 & 26 & 17.55 & 51.77 & $N$ & 179.90 & N & 83.2 & $F$ & & \\
\hline 20 & 3 & 35 & 44.07 & 52.26 & $N$ & 179.05 & $W$ & 125.0 & D & & \\
\hline 2 ? & 4 & 11 & 25.03 & 50.91 & $N$ & 179.22 & $E$ & 27.9 & $F$ & & \\
\hline $2 n$ & 4 & 33 & 2.50 & 51.30 & $N$ & 179.94 & $W$ & 40.0 & $T$ & 5.1 & \\
\hline 20 & 4 & 48 & 43.41 & 51.02 & $N$ & 179.95 & $E$ & 22.4 & $F$ & . & \\
\hline 20 & 5 & 14 & $23.8 ?$ & 51.06 & $N$ & 179.98 & $E$ & .3 & $F$ & & \\
\hline 20 & 5 & 46 & $4 \Gamma .65$ & 51.57 & $N$ & 179.15 & $E$ & 64.4 & $F$ & & \\
\hline 20 & 5 & $54^{\circ}$ & .37 .92 & 51.04 & $N$ & 179.96 & $E$ & 12.6 & $F$ & & \\
\hline 20 & 5 & 56 & 56.98 & 50.88 & $N$ & 179.92 & W & 30.0 & $F$ & & . \\
\hline 20 & 7 & 50 & 31.54 & 51.73 & $N$ & 179.74 & $E$ & 100.0 & 0 & & \\
\hline 20 & a & 6 & 26.68 & 51.40 & $N$ & 179.76 & $E$ & 28.0 & $F$ & & \\
\hline 20 & 9 & 22 & 7.65 & 51.96 & $N$ & 179.53 & $E$ & 179.4 & $F$ & & \\
\hline 20 & 18 & 5 & 40.32 & 50.97 & $N$ & 179.81 & $E$ & 32.4 & $F$ & & \\
\hline
\end{tabular}

RAT ISLANDS, ALEUTIAN ISLANDS

RAT ISLANDS, ALEUTIAN ISLANDS

NORTH ATLANTIC RIDGE

RAT ISLANDS, ALEUTIAN ISLANDS

SOUTH OF FIJI ISLANDS

RAT ISLANDS, ALEUTIAN ISLANDS

RAT ISLANDS, ALEUTIAN ISLANDS

RAT ISLANOS, ALEUTIAN ISLANDS

DELAROF ISLANDS, ALEUTIAN ISLANOS

DELAROF ISLANDS, ALEUTIAN ISLANDS

RAT ISLANDS, ALEUTIAN ISLANDS

OELAROF ISLANOS, ALEUTIAN ISLANDS

DELAROF ISLLANDS, ALEUTIAN ISLANOS

DELAROF ISLANOS, ALEUTIAN ISLANDS

RAT ISLANOS, ALEUTIAN ISLANDS

RAT ISLANDS, ALEUTIAN ISLANDS

RAT ISLANDS, ALEUTIAN ISLANDS

DELAROF ISLANOS, ALEUTIAN ISLANDS

DELAROF ISLANOS, ALEUTIAN ISLANOS

DELAROF ISLANDS, ALEUTIAN ISLANDS

RAT ISLANDS, ALEUTIAN ISLANDS

DELAROF ISLANDS, ALEUTIAN ISLANDS

RAT ISLANDS, ALEUTIAN ISLANOS

RAT ISLANDS, ALEUTIAN ISLANDS

RAT ISLANDS, ALEUTIAN ISLANDS

RAT ISLANDS, ALEUTIAN ISLANDS

DELAROF ISLANDS, ALEUTIAN ISLANDS

RAT ISLANDS, ALEUTIAN ISLANDS

RAT ISLANDS, ALEUTIAN ISLANDS

RAT ISLANDS, ALEUTIAN ISLANDS

RAT ISLANDS, ALEUTIAN ISLANDS 


\begin{tabular}{|c|c|c|c|c|c|c|c|c|c|c|c|}
\hline $\begin{array}{l}\text { NOV } \\
1970\end{array}$ & ORI & $\begin{array}{l}\text { GIN } \\
\text { IGMT }\end{array}$ & $\begin{array}{l}\text { TIME } \\
(T)^{1}\end{array}$ & " LAT & $\cdots$ & LONG & & $\begin{array}{l}\text { OEPTH* } \\
\text { (KM) }\end{array}$ & & $M B$ & MS \\
\hline 20 & 11 & 13 & 7.30 & 51.39 & N & $178.29 \mathrm{~W}$ & $w$ & $34.0 \mathrm{~T}$ & $T$ & 5.1 & \\
\hline 20 & 12 & 13 & 4.52 & 50.96 & $N$ & $178.64 \mathrm{E}$ & E & $25.5 \mathrm{~F}$ & $\mathbf{F}$ & ' & ' \\
\hline 20 & 12 & 443 & $31.90^{\circ}$ & 49.99 & $\mathbf{N}$ & $159.26 \mathrm{E}$ & $E$ & $35.0 \mathrm{~T}$ & $\mathbf{T}$ & 4.6 & \\
\hline 20 & 13 & 46 & 52.80 & 28.12 & N & $142.48 \mathrm{E}$ & $E$ & $33.0 \mathrm{~T}$ & $\mathbf{T}$ & 5.5 & 5.4 \\
\hline 20 & 15 & 553 & $33.90^{\prime}$ & 51.17 & $N$ & $178.30 \mathrm{~W}$ & w & $31.0 \mathrm{~T}$ & $\mathbf{T}$ & 3.9 & \\
\hline 20 & 16 & 14 & 14.77 & 52.02 & $\mathrm{~N}$ & $178.70 \mathrm{E}$ & $E$ & $73.4 \mathrm{~F}$ & $F$ & & \\
\hline 20 & 16 & 55 & 9.99 & 52.38 & $N$ & $179.69 \mathrm{~W}$ & $w$ & $147.0 \mathrm{~F}$ & $F$ & & \\
\hline 20 & 17 & 21 & 45.90 & 52.60 & $N$ & 176.11 & N & $276.0 \mathrm{~T}$ & $T$ & & \\
\hline 20 & 18 & 41 & $21.80^{13}$ & 51.50 & $N$ & $179.42 \mathrm{E}$ & $E$ & $23.8 \mathrm{~F}$ & $F$ & & \\
\hline 20 & 19 & 59 & 24.85 & 51.38 & $\dot{N}$ & $178.80 \mathrm{E}$ & $E$ & 50.00 & D & & \\
\hline 20 & 21 & 12 & 8.60 & 50.91 & $N$ & 173.11 & $w$ & $62.0 \mathrm{~T}$ & $T$ & & \\
\hline 20 & 21 & 53 & 25.00 & 51.12 & $N$ & $178.66 \mathrm{E}$ & $E$ & $28.3 \mathrm{~F}$ & $\mathbf{F}$ & & \\
\hline $20^{\circ}$ & 22 & 9 & 37.77 & $51.54^{\circ}$ & $\cdot \mathbf{N}$ & $177.65 \cdot E$ & $E$ & $50.2 \mathrm{~F}$ & $\mathbf{F}$ & & \\
\hline 20 & 22 & 36 & 2.89 & 51.15 & $N$ & $178.67 \mathrm{E}$ & $E$ & $34.8 \mathrm{~F}$ & $F$ & & \\
\hline 20 & 23 & 33 & $1.30^{\circ}$ & 50.07 & $N^{\prime}$ & $177.47^{\circ} \mathrm{E}$ & $E$ & $33.0 \mathrm{~T}$ & $T$ & & : \\
\hline 20 & 23 & 53 & 53.95 & 51.01 & $N$ & $179.51 \mathrm{E}$ & $E$ & 25.00 & D. & & \\
\hline 21 & 1 & 112 & 44.28 & $51: 46$ & $N$ & $179.66 \mathrm{~W}$ & $W$ & $66.7 \mathrm{~F}$ & $F$ & & \\
\hline 21 & 1 & 45 & $57.43^{\circ}$ & 51.33 & $\mathrm{~N}$ & $179.94 \mathrm{E}$ & $E$ & $9.1 \mathrm{~F}$ & $F$ & & \\
\hline 21 & 3 & $22^{\prime}$ & 21.03 & 51.72 & $N$ & $179.90 \mathrm{E}$ & $E$ & $21.0 \mathrm{~F}$ & $F$ & 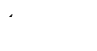 & \\
\hline 21 & $4^{\circ}$ & 11 & 33.84 & 51.46 & $N$ & $178.93 \mathrm{E}$ & $E$ & $45.5 \mathrm{~F}$ & $F$ & & \\
\hline 21 & 9 & 26 & 12.56 & $52: 08$ & $N$ & 178.10 & $E$ & $147.1 \mathrm{~F}$ & $F$ & 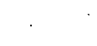 & \\
\hline 21 & 9 & 34 & 44.54 & 51.07 & $N$ & $179.00 \mathrm{E}$ & $E$ & $27.6 \mathrm{~F}$ & $F$ & & \\
\hline 21 & 10 & 53 & 14.80 & 51.88 & $\dot{N}$ & $178.70 \mathrm{E}$ & $\mathrm{E}$ & $85.3 F$ & $\mathrm{~F}$ & & \\
\hline 21 & 12 & 47 & 58.50 & 52.04 & $N$ & 176.23 & $w$ & $132.0 T$ & $T$ & & \\
\hline 21 & 15 & 13 & 32.97 & 51.52 & $N$ & $179.36:$ & $E$ & $70.3 \mathrm{~F}$ & $\mathbf{F}$ & & \\
\hline 21 & 19 & 26 & 28.00 & 56.95 & $\mathrm{~N}$ & 156.98 & $w$ & $90.0 T$ & $T$ & $4 \cdot 6$ & \\
\hline 21 & 19 & 32 & 47.80 & 52.32 & $N$ & 178.55 & $N$ & 170.0 .7 & $\mathbf{T}$ & 4.6 & \\
\hline 21 & 22 & 20 & 27.63 & 50.65 & $\mathbf{N}$ & $178.05 \mathrm{E}$ & $E$ & $50.0 \mathrm{C}$ & D & & $\cdot$ \\
\hline 22 & 1 & 6 & 44.23 & 51.93 & $N$ & 179.20 & W & $34.9 F$ & $F$ & & \\
\hline 22 & 19 & 32 & 53.36 & 51.13 & $\mathbf{N}$ & 179.21 & $E$ & $27.8 F$ & $\mathbf{F}$. & & 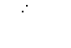 \\
\hline 23 & 1 & 13 & $57.80^{\circ}$ & $61.49^{\circ}$ & $N$ & 149.85 & $w$ & 48.0 & $\mathbf{T}$ & 4.0 & \\
\hline
\end{tabular}

\section{GEOGRAPHIC REGION}

DELAROF ISLANDS, ALEUTIAN ISLANDS

RAT ISLANOS, ALEUTIAN ISLANDS

KURIL ISLANDS REGION

BONIN ISLANDS REGION

DELAROF ISLANOS, ALEUTIAN ISLANDS

RAT ISLANOS, aLEUTIAN ISLANOS

DELAROF ISLANDS, ALEUTIAN ISLANDS

ANDREANOF ISLANDS, ALEUTIAN IS.

RAT ISLANDS, ALEUTIAN ISLANOS

RAT ISLANDS, ALEUTIAN ISLANDS

ANDREANOF ISLANOS, ALEUTIAN IS.

RAT ISLANDS, ALEUTIAN ISLANDS

RAT ISLANDS, ALEUTIAN ISLANDS.

RAT ISLANDS, ALEUTIAN ISLANDS

RAT ISLANDS, ALEUTIAN ISLANDS

RAT ISLANOS, ALEUTIAN ISLANDS

DELAROF ISLANDS, ALEUTIAN ISLANOS

RAT ISLANDS, ALEUTIAN ISLANOS

RAT ISLANDS, ALEUTIAN ISLANOS

RAT ISLANDS, ALEUTIAN ISLANDS

RAT ISLANOS, ALEUTIAN ISLANOS

RAT ISLANDS, ALEUTIAN ISLANDS

RAT ISLANDS, ALEUTIAN ISLANOS

ANDREANOF ISLANOS, ALEUTIAN IS.

RAT ISLANDS, ALEUTIAN ISLANDS

ALASKA PENINSULA

DELAROF ISLANDS, ALEUTIAN ISLANDS

RAT'ISLANDS, ALEUTIAN ISLANDS

DELAROF ISLANDS, ALEUTIAN ISLANDS

RAT ISLANDS, ALEUTIAN ISLANDS

SOUTHERN ALASKA 
NOV HYPOCENTER SUMMARY

\begin{tabular}{|c|c|c|c|c|c|c|c|c|c|c|c|}
\hline $\begin{array}{l}\text { NOV } \\
1970\end{array}$ & OR.I & $\begin{array}{l}\text { GIN } \\
\text { CGM }\end{array}$ & $\begin{array}{l}1 \text { TIME } \\
\text { (T) }\end{array}$ & LAT & & LONG & & $\begin{array}{c}\text { DEPTH } \\
\text { (KH) }\end{array}$ & & $M \theta$ & MS \\
\hline 2.3 & $2 \pi$ & 3 & 18.59 & 51.41 & $N$ & 179.14 & $E$ & 53.8 & $F$ & & \\
\hline 24 & 5 & 6 & 41.40 & 47.44 & $N$ & 152.49 & $E$ & 136.0 & $T$ & 5.3 & \\
\hline 24 & 11 & $2 A$ & 58.58 & 51.17 & $N$ & 179.02 & $\mathrm{E}$ & 23.9 & $F$ & & \\
\hline 25 & 3 & 34 & 28.22 & $51 \cdot 76$ & $N$ & 178.68 & $E$ & 83.7 & $F$ & & \\
\hline 25 & $P$ & 32 & 28.97 & 51.52 & $N$ & 179.26 & $E$ & 62.9 & $F$ & & \\
\hline 26 & 5 & 23 & 52.60 & 51.13 & $\mathrm{~N}$ & 178.23 & $w$ & 26.0 & $T$ & 3.7 & \\
\hline 28 & 14 & $4 ?$ & 39.39 & 51.22 & $N$ & 179.46 & $E$ & 41.5 & $F$ & & \\
\hline EA & 20 & 22 & 50.60 & 4.13 & $\mathrm{~s}$ & 142.90 & $E$ & 11.4 .0 & $T$ & 5.8 & \\
\hline 29 & 4 & 3 & 15.37 & 51.26 & $N$ & 177.95 & $E$ & $50 \cdot 0$ & D & & \\
\hline 29 & $1 P$ & 45 & 44.20 & 50.74 & $N$ & 176.90 & $W$ & 47.0 & $T$ & 3.8 & \\
\hline 29 & 20 & 35 & 55.50 & 51.43 & $N$ & 179.56 & $w$ & 24.0 & $F$ & & \\
\hline 30 & 1 & 4 & 41.00 & 51.74 & $N$ & 179.02 & $E$ & 194.8 & $\mathbf{F}$ & & \\
\hline 30 & 3 & 29 & 45.06 & 51.13 & $N$ & 178.83 & $E$ & 25.6 & $\mathbf{F}$ & & \\
\hline 30 & 18 & 18 & $40 \cdot 30$ & 51.19 & $N$ & 178.20 & W & 27.0 & $\mathbf{T}$ & & \\
\hline 30 & $1 \mathrm{~A}$ & 19 & 6.10 & 50.71 & $N$ & 150.59 & $\mathbf{W}$ & 50.0 & $\mathbf{T}$ & 4.0 & \\
\hline
\end{tabular}

PAGE 70-11-63

GEOGRAPHIC REGION

RAT ISLANDS, ALEUTIAN ISLANDS

KURIL ISLANDS

RAT ISLANDS, ALEUTIAN ISLANOS

RAT ISLANDS, ALEUTIAN ISLANDS

RAT ISLANDS, ALEUTIAN ISLANDS

ANDREANOF ISLANDS, ALEUTIAN IS.

RAT ISLANDS, ALEUTIAN ISLANDS

NEW GUINEA

RAT ISLANDS, ALEUTIAN ISLANDS

ANDREANOF ISLANOS, ALEUTIAN IS.

DELAROF ISLANDS, ALEUTIAN ISLANOS

RAT ISLANOS, ALEUTIAN ISLANDS

RAT ISLANDS, ALEUTIAN ISLANDS

ANDREANOF ISLANDS, ALEUTIAN IS.

KENAI PENINSULA, ALASKA

\footnotetext{
* F = FPEE DEPTH SOL.UTION 
PAGE $\quad 70-11-64$

\section{SEISMICITY MAPS}

ALEUTIAN EARTHQUAKES WHICH WERE LOCÁTEO DURING THE MONTH BY THE ALEUTIAN SEISMIC NETWORK AND BY THE WORLD NETHORK, OF SEISMOLOGICAL STATIONS REPORTING TO THE NATIONAL OCEAN SURVEY (NOS) ARE PLOTTED ON SIX MAPS COVERING THE ALEUTIAN ISLANDS FROM THE NEAR ISLANDS TO UNIMAK ISLAND' 170 DEGREES EAST LONGITUDE TO 160 DEGRES WEST LONGITUDE).

THE BASE MAPS ARE MODIFIED VERSIONS OF LARGE PLATES PUBLISHEO BY THE NOS AS PART OF ESSA MONOGRAPH NO. 3 (BATHYMETRY OF THE ALEUTIAN ARC). THE TRANSVERSE MERCATOR PROJECTION, IN MHICH AREAL DISTORTION IS SMALL AND BEARINGS REMAIN TRUE, WAS EMPLOYED.

EPICENTERS WERE PLOTTED AUTOMATICALLY BY A FLAT-BED INCREMENTAL PLOTTER TO PRODUCE PLATES WHICH HERE USED TO OVERPRINT THE BASB MAPS. REGISTRATION MARKS ALONG THE MARGINS OF THE MAPS ALLOH AN ESTIMATION OF THE PRECISION OF PRINTING REGISTRATION.

EACH PLOTTED SYMBOL, CENTERED UPON THE EPICENTRAL COORDINATES, REPRESENTS ONE EARTHQUAKE. THE TYPE OF SYMBOL DENOTES THE DEPTH-OF-FOCUS CLASS OF THE SEISMIC EVENT. THE BODY-WAVE MAGNITUde (MB) IS INDICATED BY THE SYMBOL SIZE, HHICH VARIES CONTINUOUSLY ACCOROING TO THE RELATIONSHIP

$$
\text { SYMBOL SIZE (INCHES) }=.07+(M B-2.5) * .10
$$

EVENTS hHOSE MAGNITUDES ARE UNDETERMINED OR LESS THAN 2.5 ARE PLOTTED AS 07 INCH SYMBOLS. A LEGEND BLOCK DEPICTING THE FIVE DEPTH-OF-FOCUS CLASSES AND A Range of magnitudes is provided on the attu plate. 


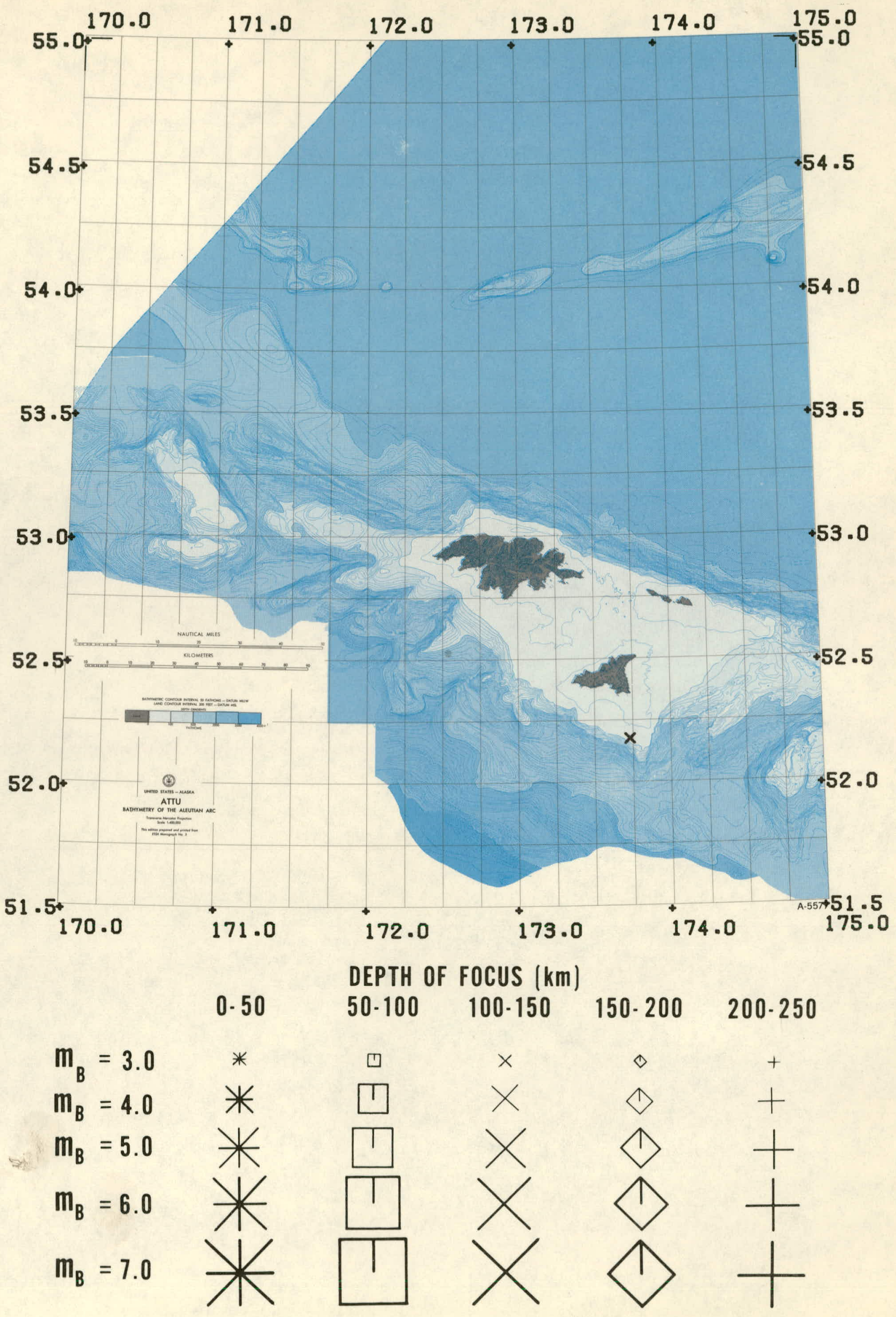




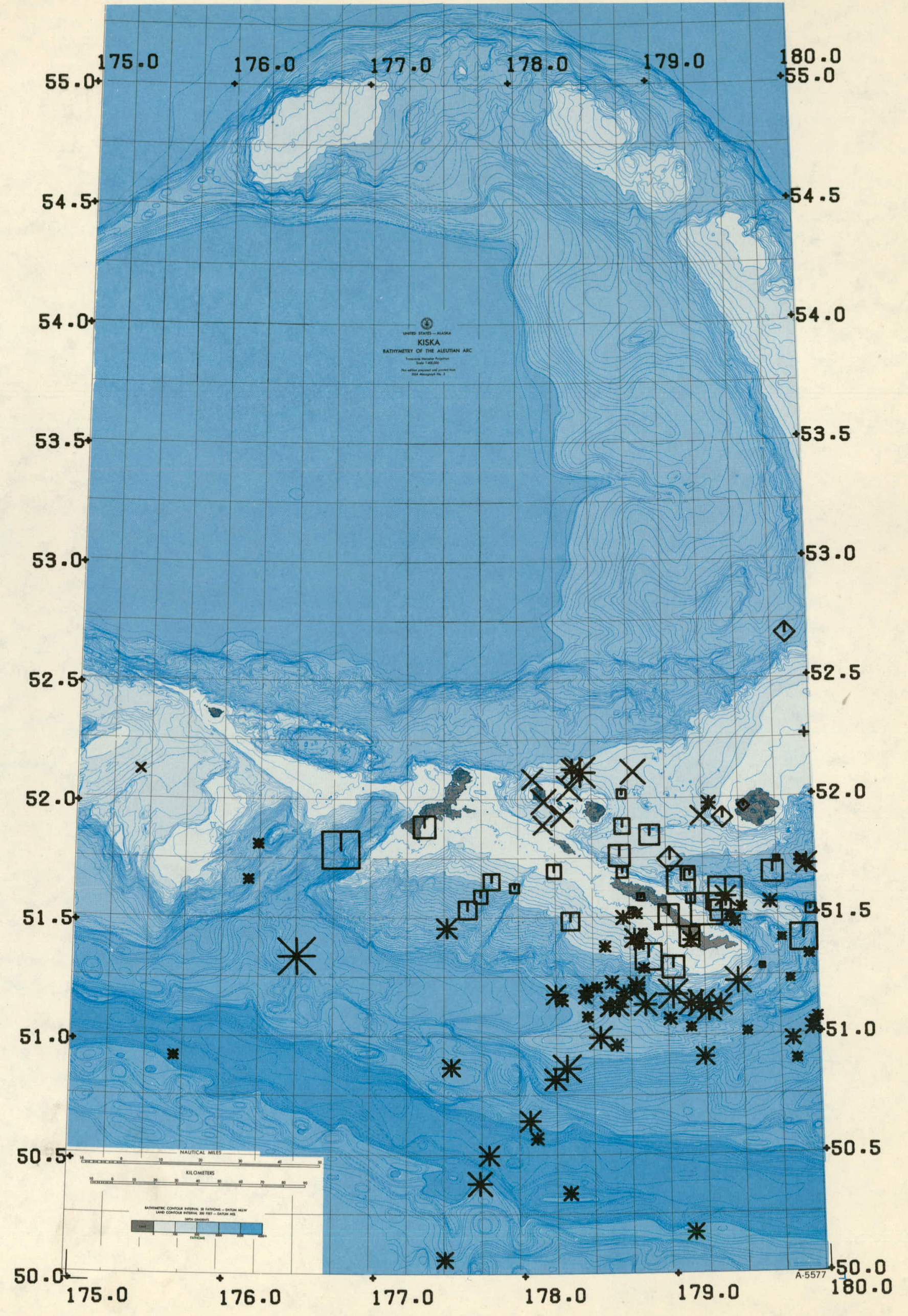




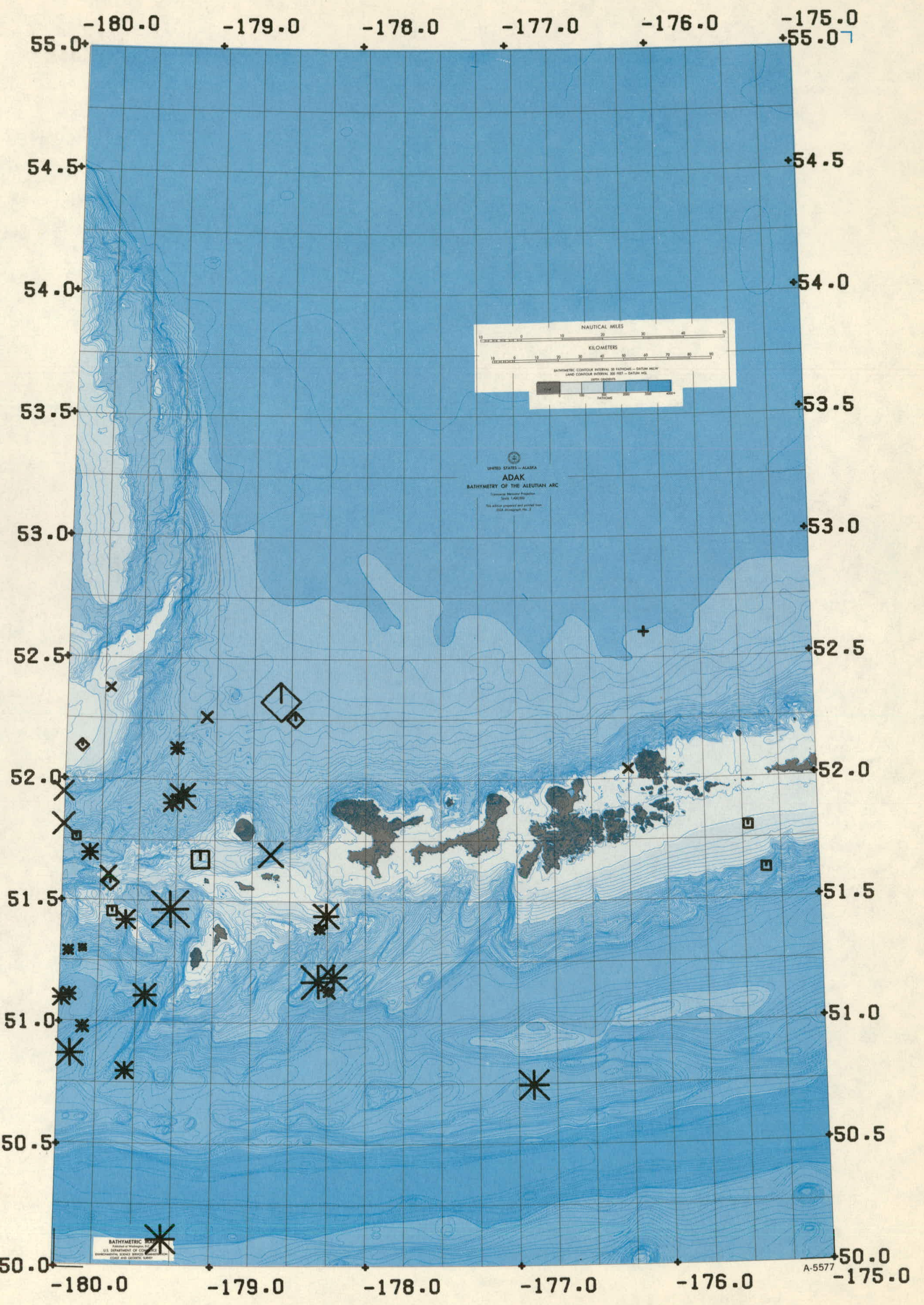




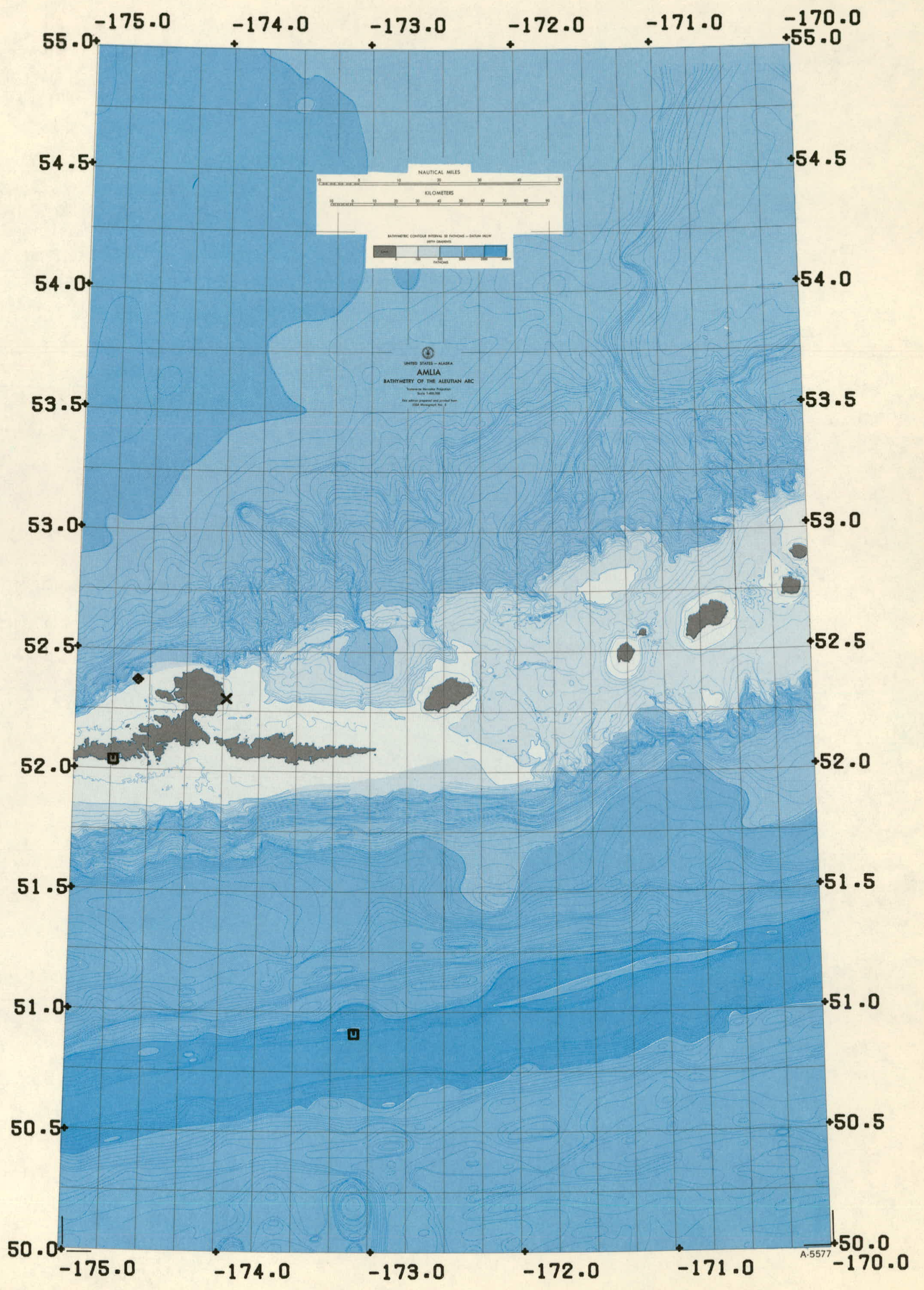




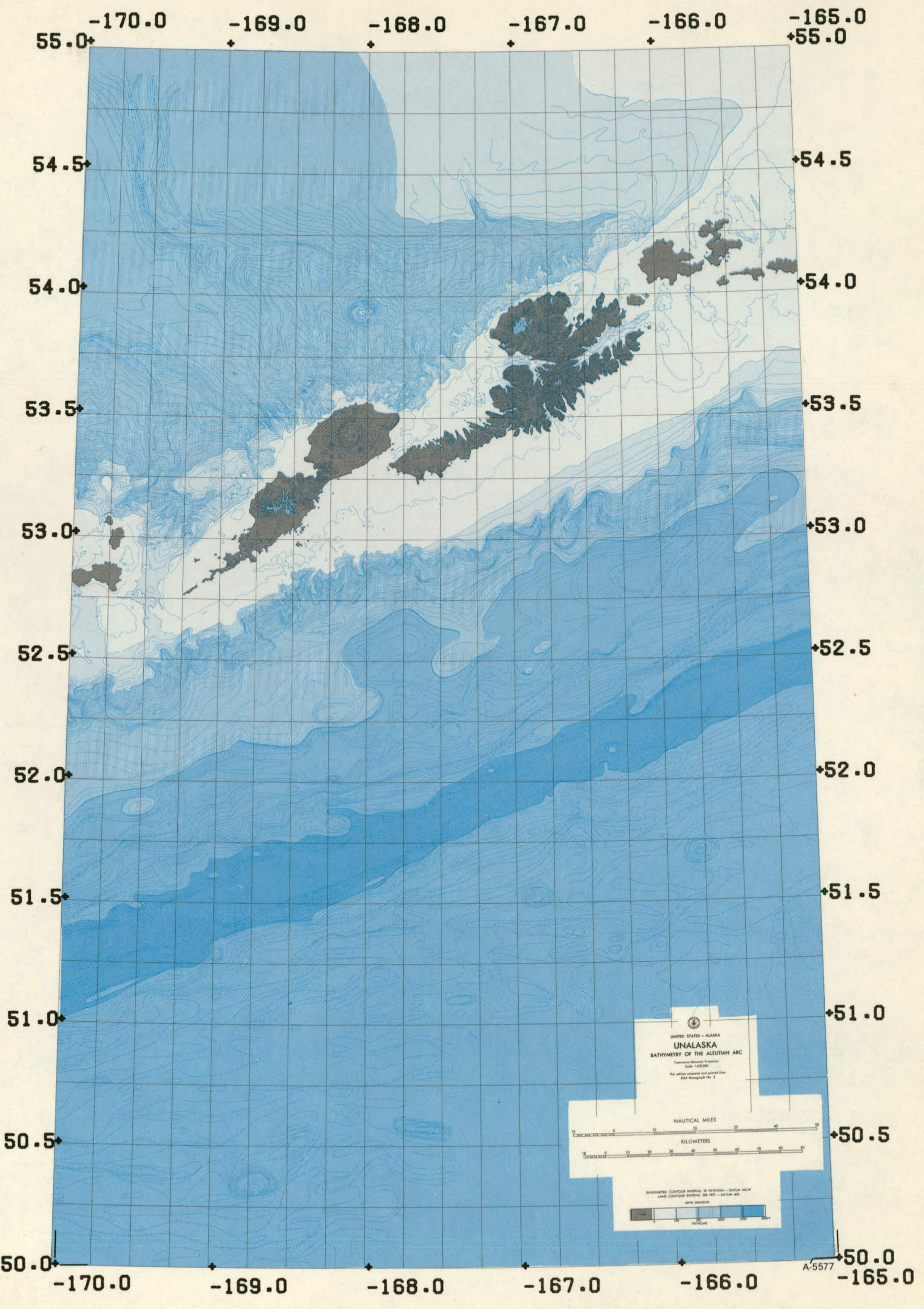




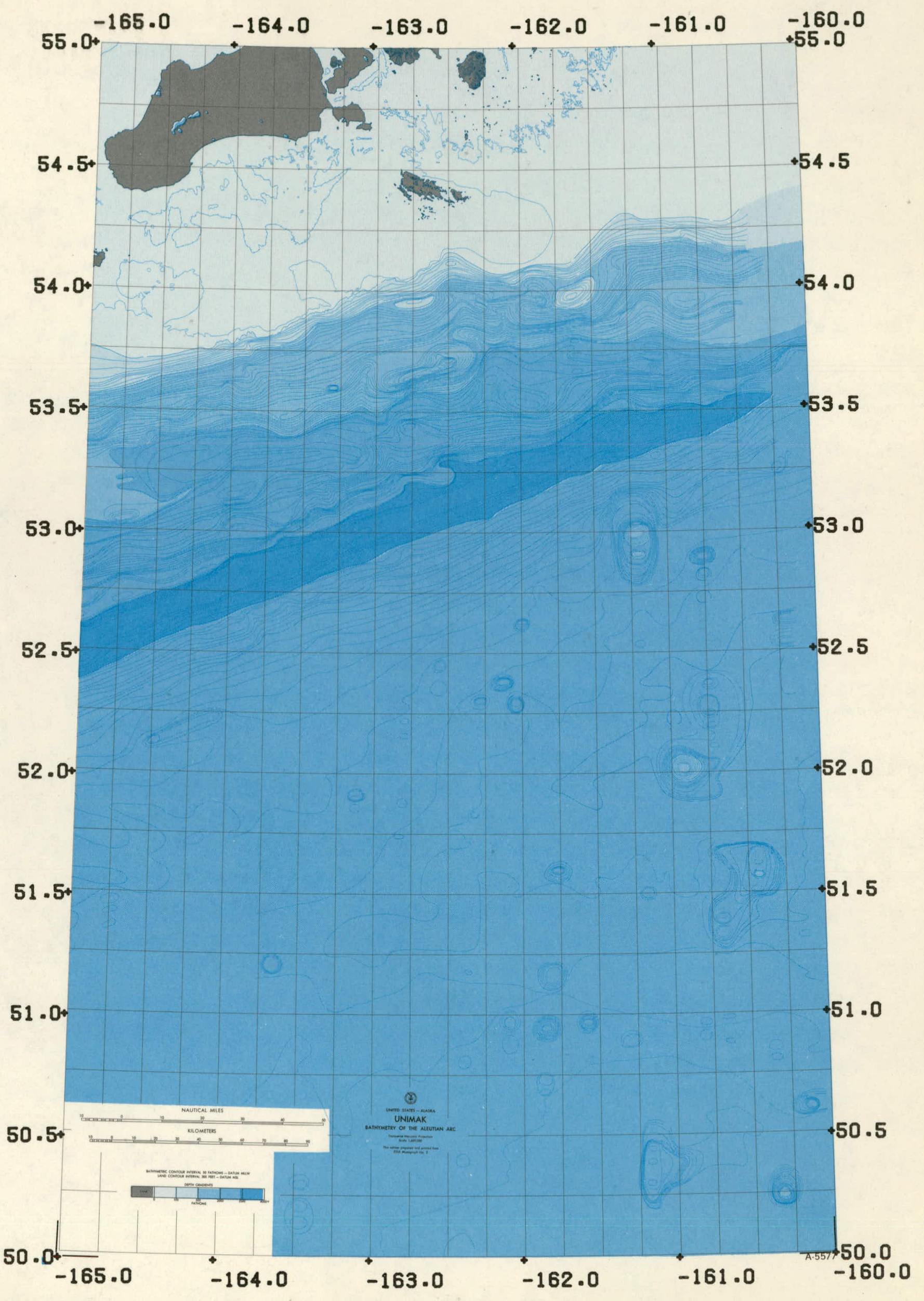

